Universidade de São Paulo

Instituto de Física

\title{
Análogos Cosmológicos dos Efeitos Schwinger Estático e Oscilatório
}

\author{
Elisa Gouvêa Mauricio Ferreira
}

Orientador: Prof. Dr. Luis Raul Weber Abramo

Dissertação de Mestrado apresentada ao Instituto de Física da Universidade de São Paulo para a obtenção do título de Mestre em Ciências.

Banca Examinadora:

Prof. Dr. Luis Raul Weber Abramo (IFUSP)

Prof. Dr. Rogério Rosenfeld (UNESP)

Profa. Dra. Maria Carolina Nemes (UFMG)

São Paulo

2009 
FICHA CATALOGRÁFICA

Preparada pelo Serviço de Biblioteca e Informação do Instituto de Física da Universidade de São Paulo

Ferreira, Elisa Gouvêa Mauricio

Análogos cosmológicos dos efeitos Schwinger estático e oscilatório. São Paulo, 2009.

Dissertação (Mestrado) - Universidade de São Paulo. Instituto de Física, Departamento de Física Matemática.

Orientador: Prof. Dr. Luís Raul Weber Abramo

Área de Concentração: Física

Unitermos: 1. Cosmologia; 2.Universo primordial;

3. Teoria quântica de campo. 


\section{Resumo}

Neste trabalho estudamos os efeitos quânticos de criação de partículas nos contextos cosmológico e da eletrodinâmica clássica. Com o estudo da criação de partículas em campo elétricos externos constantes e oscilatórios, os conhecidos efeitos Schwinger estático e oscilatório, respectivamente, foi possível estudar a polarização do vácuo quântico e a conseqüente criação de partículas do vácuo. Esse é um efeito não-perturbativo no contexto da eletrodinâmica e sua observação seria de grande importância para validar essa teoria nesse regime.

Esse efeito, no entanto, ainda não foi detectado experimentalmente, uma vez que são necessários campos elétricos muito fortes no caso estático. Contudo, no caso oscilatório existe um experimento em andamento para a deteç̧ão de um efeito quântico análogo, o chamado efeito Casimir dinâmico. A verificação do efeito Casimir dinâmico é importante para a validação do efeito Schwinger oscilatório, dado que o movimento das condições de contorno é equivalente à aplicação de um campo externo.

Finalmente, foi estudada a criação de partículas em um contexto cosmológico, sendo o caso estático a criação de partículas na era inflacionária do universo. O caso dinâmico corresponde ao período de reaquecimento ou, mais precisamente, em seu período inicial conhecido como pré-aquecimento, em que a criação de partículas é mais eficiente. A criação de partículas na era inflacionária ocorre devido à expansão acelerada do universo. Por meio de uma transformação canônica simples foi possível observar que o mecanismo de criação de partículas nesse período é o mesmo da criação de partículas do efeito Schwinger estático. Da mesma forma, para o pré-aquecimento, em que a criação de partículas é dada pela oscilação do inflaton em torno de seu mínimo do potencial, o mecanismo de criação resume-se a um mecanismo de ressonância paramétrica descrito por uma equação de Mathieu. Esse é exatamente o mesmo mecanismo que descreve a criação de partículas no caso do efeito Schwinger oscilatório.

Dessa forma, os mecanismos de criação de partículas nos casos do efeitos Schwinger estático e oscilatório são análogos aos mecanismo de criação inflacionário e do préaquecimento. A deteç̧ão experimental desse efeito em um experimento terrestre seria uma grande confirmação para esses mecanismos cosmológicos. 


\begin{abstract}
In this work we study the quantum effects of particle creation in the cosmological context and of the classical electrodynamics. With the studies of the particle creation in constant and oscillatory external electrical fields, the known static and oscillatory Schwinger effects, respectively, it was possible to study the polarization of the quantum vacuum and the consequently creation of particles from the vacuum. This is a non perturbative effect in the context of the electrodynamics and its observation would be of great importance to validate this theory in this regime.

This effect, however, was not yet experimentally detected, since very strong electric fields are necessary in the static case. However, in the oscillatory case there is an experiment currently in development for the detection of an analog quantum effect called dynamical Casimir effect. The verification of the dynamical Casimir effect is important for the validation of the oscillatory Schwinger effect, since the movement of the boundary conditions is equivalent to the application of a external field.

Finally, it was studied the creation of particles in a cosmological context, being the static case the creation of particles in the inflationary epoch of the universe. The dynamical case corresponds to the reheating period or, more precisely, its initial stage known as preheating, in which the creation of particles is more efficient. The creation of particles in the inflationary epoch occurs due to the accelerated expansion of the universe. By means of a simple canonical transformation it was possible to observe that the mechanism of particle creation in this period is the same of the one in Schwinger effect. In the same way, for the preheating, in which the particle creation is given by the oscillation of the inflaton field around the minimum of its potential, the mechanism of creation happens due to the parametric resonance described by a Mathieu equation. This is exactly the same mechanism that describes the oscillatory Schwinger effect.

In this way, the mechanisms of particle creation in the cases of the static and oscillatory Schwinger effects are analogs to the creation mechanisms from inflation and from preheating. The experimental detection of this effect in a terrestrial experiment would be a confirmation for this cosmological mechanisms.
\end{abstract}




\section{Estrutura da Dissertação}

A presente dissertação pretende fazer uma analogia entre mecanismos de criação de partículas cosmológicos e na eletrodinâmica. Dessa forma, mostra-se necessário a introdução dos conceitos básicos tanto de cosmologia, quanto de teoria quântica de campos e eletrodinâmica. Esses dois últimos aparecem conforme necessários nos capítulos em que estudamos os efeitos de criação de partículas na eletrodinâmica. Contudo, como esta é um dissertação da área de cosmologia, a introdução de seus conceitos básicos foi detalhadamente revisada. Com isso, os Capítulos 2 e 3 ( e o início do Capítulo 4) podem ser omitidos da leitura para aqueles que os tiverem como matéria conhecida.

\section{Notação}

Apresentamos aqui a notação que será utilizada ao longo da dissertação. Primeiramente, destacamos que utilizaremos o sistema de unidades no qual $c=\hbar=k_{B}=1$, em que $c$ é a velocidade da luz no vácuo e $k_{B}$ é a constante de Boltzmann. Também temos que índices gregos variam de 0 a 3 e índices latinos variam de 1 a 3 .

Abaixo apresentamos mais algumas notações utilizadas.

\section{Geral}

$*$

† ou h.c.

$\partial_{\mu}$ ou $\frac{\partial}{\partial_{\mu}}$ ou,$\mu$

$D_{\mu} \mathrm{ou} ; \mu$

$\square \equiv g^{\mu \nu} D_{\mu} D_{\nu}$

$=D_{\mu} D^{\mu}$

$\mathrm{Re} / \mathrm{Im}$

$\operatorname{Tr}$

$\begin{array}{ll}{[A, B]=A B-B A} & \text { Comutador } \\ \{A, B\}=A B+B A & \text { Anticomutado }\end{array}$

$\{A, B\}=A B+B A \quad$ Anticomutador

Métrica de Minkowski:

$g_{\mu \nu}=\operatorname{diag}(1,-1,-1,-1)$

Matriz $\gamma$ :

$\gamma^{\mu} \gamma^{\nu}+\gamma^{\nu} \gamma^{\mu}=2 g^{\mu \nu}$

$\gamma^{5}=i \gamma^{0} \gamma^{1} \gamma^{2} \gamma^{3}$

D'Lambertiano

Traço

$$
\sigma_{\mu \nu}=\frac{i}{2}\left[\gamma_{\mu}, \gamma_{\nu}\right]
$$

complexo conjugado

Hermitiano conjugado Convenção de soma de Einstein:

Derivada parcial

$p q=p_{\mu} q^{\mu}$

Derivada covariante

Distribuições

Parte real/imaginária Função degrau

$\theta(x-y)=\left\{\begin{array}{l}0, \text { se } x<y \\ 1, \text { se } x>y\end{array}\right.$

\section{Função delta}

$\delta^{D}(x-y)=\frac{1}{(2 \pi)^{D}} \int d^{D} q e^{i q(x-y)}$

\section{Distribuição de Pauli-Jordan}

$\triangle\left(x-y ; m^{2}\right)=\frac{-i}{(2 \pi)^{4}} \int d^{4} q e^{-i q(x-y)} \varepsilon\left(q^{0}\right) \delta\left(q^{2}-m^{2}\right)$ 


\section{Agradecimentos}

Agradeço primeiramente ao meu orientador Prof. Dr. Luís Raul Weber Abramo, por ter me orientado, pela paciência e principalmente por me ensinar como fazer ciência com qualidade, esforço e dedicação. Agradeço também ao apoio dos alunos de seu grupo pelas conversas, ajudas e seminários que foram sempre produtivos e principalmente ao Thiago Pereira por ter contribuído com discussões muito ricas sobre o assunto dessa dissertação.

Agradeço imensamente à minha família. Aos meus pais e irmãos que sempre compreenderam e apoiaram a minha ausência sabendo que estava em busca de meu sonho. Muito obrigada por terem feito tudo ser possível, me amado e apoiado sempre. Amo vocês e sinto como se estivesse sempre ao lado de vocês. Agradeço também ao vovô Ferreira e a vovó Rosa que me trataram como sua filha sempre e me apoiaram nessa jornada.

Agradeço aos meus amigos de graduação e de sempre: Bruno, Fabio, Felipe, Herman, João (recente, mas sempre presente), Pedro, Silas, Thiago, Walter e William. Mas agradeço principalmente a Eduardo Toshio, meu irmão e amigo incondicional.

Ao Departamento de Física Matemática e a Simone e Amélia. Pelo financiamento que possibilitou essa pequisa, agradeço ao $\mathrm{CNPq}$.

Por fim, agradeço especialmente ao meu Will, meu amor, companheiro e melhor amigo. Sempre me apoiando, me incentivando e estando ao meu lado. Obrigada pelo seu amor incondicional e principalmente por estar sempre ao meu lado. Não teria chegado aqui sem você. Amo você e sempre vou te amar! 
A meu Will e à minha família.

Ao vovô: saudades... 


\section{Sumário}

1 Introdução $\quad 9$

2 Modelo Cosmológico Padrão $\quad 15$

2.1 Previsões geométricas do MCP . . . . . . . . . . . . . . 16

2.1 .1 Estrutura do universo . . . . . . . . . . . . . . 16

2.1 .2 Expansão do universo . . . . . . . . . . . . . . . . . 18

2.1.3 Evolução do universo . . . . . . . . . . . . . . . . . . 21

2.1.4 Horizontes . . . . . . . . . . . . . . . 25

2.2 Descrição do universo pelo MCP e o universo jovem . . . . . . . . . . . 27

2.3 Testes do MCP . . . . . . . . . . . . . . . . . 30

2.3.1 A Lei de Hubble . . . . . . . . . . . . . . . . . . 30

2.3.2 Abundância dos Elementos Leves . . . . . . . . . . . . . . 31

2.3.3 A Radiação Cósmica de Fundo . . . . . . . . . . . . . . . 32

2.4 Problemas do MCP . . . . . . . . . . . . . . . . . 33

2.4 .1 Problema da planura . . . . . . . . . . . . . . 33

2.4 .2 Problema do horizonte . . . . . . . . . . . . . . 34

2.4.3 Problema da origem das estruturas . . . . . . . . . . . . 36

2.4.4 Problema dos monopolos magnéticos . . . . . . . . . . . . . 36

3 Inflação $\quad 37$

3.1 Motivação . . . . . . . . . . . . . . . . . . . 38

3.1 .1 Problema da planura . . . . . . . . . . . . . . . 40

3.1 .2 Problema do horizonte . . . . . . . . . . . . . . 41

3.1.3 Problema dos monopolos magnéticos . . . . . . . . . . . . 42

3.1.4 O problema da origem das estruturas . . . . . . . . . . . 43

3.2 O paradigma inflacionário: a inflação e o inflaton . . . . . . . . . . . 43

3.2.1 Regime de rolagem lenta (Slow-Roll) . . . . . . . . . . . . . . 45

3.2 .2 Número de $e$-folds . . . . . . . . . . . . . . . 47

3.2 .3 Inflação caótica . . . . . . . . . . . . . . . . . . 48

3.3 Pré-aquecimento e Reaquecimento . . . . . . . . . . . . . . . 52

3.3.1 Teoria Elementar do Reaquecimento . . . . . . . . . . . 54 


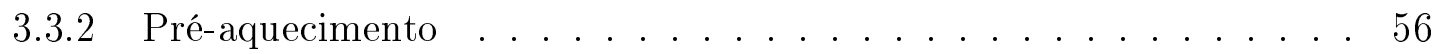

4 Teoria das Perturbações Cosmológicas $\quad 58$

4.1 Teoria Clássica das Perturbações Cosmológicas . . . . . . . . . . . . . . . 59

4.1.1 Teoria das Perturbações na relatividade geral . . . . . . . . . . 59

4.2 Teoria Quântica das Perturbações Cosmológicas: flutuações quânticas e o "nascimento" das perturbações cosmológicas . . . . . . . . . . . . . . 74

4.2.1 Quantização . . . . . . . . . . . . . . . . 78

4.2.2 Espectro de Potências . . . . . . . . . . . . . . 83

5 Efeito Schwinger Estático $\quad 87$

5.1 Formalismo geral e quantização canônica . . . . . . . . . . . . . . . . . 89

5.2 Evolução Temporal . . . . . . . . . . . . . . . . . . . . 96

5.2 .1 Dinâmica via teoria de espalhamento . . . . . . . . . . . 97

5.2.2 Dinâmica via transformadas de Bogoliubov . . . . . . . . . . . . 106

6 Efeito Schwinger Dinâmico $\quad 119$

6.1 Efeito Schwinger Oscilatório via teoria de espalhamento . . . . . . . . . . 122

6.1.1 Tentativas e propostas experimentais . . . . . . . . . . . 132

6.2 Efeito Schwinger Oscilatório via transformadas de Bogoliubov . . . . . . 133

6.2.1 Formalismo dos estados squeezed . . . . . . . . . . . . . . 141

6.3 Efeito Casimir Dinâmico . . . . . . . . . . . . . . . . . . . . . 142

6.3.1 Quantização do campo escalar em 1+1 dimensões com condições de contorno . . . . . . . . . . . . . . . . . . 144

6.3.2 O campo eletromagnético em uma cavidade dinâmica . . . . . . . 147

6.3.3 Experimentos . . . . . . . . . . . . . . . . . 152

7 Análogos Cosmológicos $\quad 157$

7.1 Criação de Partículas na Inflação e o Efeito Schwinger Estático . . . . . . . 158

7.1.1 Campo Escalar Livre em um Espaço-Tempo Curvo . . . . . . . . . 159

7.1.2 Perturbações inflacionárias a la Schwinger . . . . . . . . . . . . . 161

7.2 Criação de Partículas no Pré-aquecimento e o Efeito Schwinger Oscilatório 168 7.2 .1 Pré-aquecimento . . . . . . . . . . . . . . 168

$\begin{array}{llr}8 & \text { Conclusão } & 187\end{array}$

A Dinâmica via formalismo da Lagrangeana efetiva (ou da ação efetiva) 198

B Integração complexa 203

C Funções de Hankel 205 


\section{Capítulo 1}

\section{Introdução}

Cosmologia é o ramo da física que estuda a estrutura, a expansão e a evolução do universo, descrevendo desde sua origem até seu futuro. Como um ramo da ciência, ela utiliza o método científico para descrever o universo como um todo. Isso quer dizer que são feitas teorias e hipóteses que podem ser testadas por observações ou experimentos. Aí reside o maior desafio da cosmologia, que retardou sua aceitação como ciência. Como o universo é um objeto que não pode ser reproduzido em laboratório, a cosmologia necessita de artifícios para poder constatar suas previsões. Para isso são utilizadas observações de suas estruturas visíveis e medições indiretas de fenômenos que são fisicamente associados a acontecimentos ocorridos no universo. Alternativamente, fazemos também o uso de analogias de sistemas cosmológicos com sistemas que são conhecidos e reprodutíveis na Terra. Nessa dissertação utilizaremos analogias para a descrição do modelo cosmológico de criação de partículas durante o período inflacionário e do reaquecimento.

A era da cosmologia moderna teve início no começo do século XX, com os trabalhos seminais de Albert Einstein. Foi possível, com o advento da teoria da relatividade, descrever o primeiro modelo matemático do universo, feito por ele em 1917 [1, 2]. Nesse modelo tínhamos um universo estático e infinito. Posteriormente, A. Friedmann e W. de Sitter (seguidos, depois, por G. Lemaître, H. Robertson e A. Walker), baseados nas equações de Einstein, mostraram que modelos do universo não-estáticos eram também possíveis.

Mas foi com E. Hubble, em 1929 [3], que se teve a primeira prova empírica de que o universo estava em expansão e essa expansão era compatível com a relatividade geral. Com essas idéias em mãos, George Gamow [5] em 1948 e seus alunos Ralph Alpher e Robert Herman [4, 6] em 1950 previram a Radiação Cósmica de Fundo (RCF), descoberta em 1965 por Arnold A. Penzias e Robert W. Wilson [7], o que lhes rendeu o prêmio Nobel. Dessa forma, a cosmologia já reunia provas bastante contundentes de um universo dinâmico e em expansão, o que significava uma ruptura do pensamento tido como definitivo pela humanidade, aquele de um universo estático e infinito. Isso permitia conceber que, no passado, o universo era muito menor e mais quente e, juntamente com a hipótese de homogeneidade e isotropia, foi construído o Modelo Cosmológico Padrão, ou Modelo 
do Big Bang Quente. Essas descobertas colocavam, assim, a cosmologia mais perto do rigor científico esperado por uma ciência física, em que as previsões eram feitas e checadas experimentalmente. Mesmo com esses avanços, nos anos 1970 ainda havia revistas científicas como a alemã Zeitschrift für Physik (Jornal de Física), que simplesmente não aceitavam quaisquer artigos com o tema cosmologia. Contudo, nos 1980, iniciou-se o que chamamos de era da cosmologia de precisão, com o lançamento do satélite COBE [8, 9], em que os dados obtidos ostentavam uma precisão muito grande, além de uma estatística muito boa. Mais recentemente, nos últimos 5 anos, o satélite WMAP [21, 22, 23] aumentou consideravelmente a qualidade dos dados obtidos, colocando-nos na era da cosmologia de alta precisão.

Os resultados desses últimos anos da cosmologia moderna permitiram-nos construir um modelo para o universo: o modelo cosmológico padrão [31, 29, 30, 32]. Ele prevê um universo homogêneo e isotrópico em largas escalas com 13,9 bilhões de anos, praticamente plano, permeado por uma radiação eletromagnética de temperatura de aproximadamente $2,75 \mathrm{~K}$ e composto por aproximadamente $4 \%$ de matéria comum, 0,01\% de radiação, $23 \%$ de matéria escura e $73 \%$ de energia escura [24]. Os experimentos recentes confirmaram muitas das hipóteses descritas nesse modelo, mas também nos permitiram verificar desvios em algumas delas. A homogeneidade e isotropia do universo são confirmadas por meio da análise da RCF. Contudo, com os dados colhidos pelo satélite WMAP, foi possível determinar um desvio de 0,001\% na temperatura da radiação em diferentes direções [24]. Isso indica flutuações iniciais quando o universo era mais jovem, sementes para a formação das estruturas em larga escala que temos hoje em dia. Podemos pensar também por que o universo é tão homogêneo e isotrópico, sendo que regiões que não são ligadas causalmente têm a mesma temperatura na RCF. Ou podemos pensar ainda por que o universo é tão plano. Todas essas questões (e outras) não são explicadas pelo modelo cosmológico padrão.

Com o intuito de resolver esses "problemas" do modelo do universo, surgiu, em 1981, desenvolvida por Alan Guth [10] ( e de maneira diferente por Starobinsky [11]), a teoria da inflação. Essa teoria surge para solucionar o problema das condições iniciais do universo. Ela prevê um período de expansão exponencial em seus estágios iniciais que seria capaz não apenas de gerar as inomogeneidades primordiais, como também conectar causalmente regiões do universo antes da inflação. Também podemos pensar que essa expansão deixa o universo enormemente grande após esse período, fazendo com que pareça plano. A inflação resolve ainda o problema da formação das estruturas cosmológicas, formadas por meio do colapso gravitacional, a partir das flutuações primordiais geradas nesse período.

A inflação pode ser descrita como movida por um campo escalar chamado inflaton. Durante esse período esse campo rola lentamente até o mínimo de seu potencial. Esse mecanismo que produz as perturbações primordiais ocorre devido às flutuações quânticas do vácuo, que significa que pares virtuais de partículas e antipartículas são criadas e aniquiladas continuamente no vácuo. Com a expansão acelerada do universo, no período 
inflacionário, esses pares virtuais se distanciam muito uns dos outros, dissociando-se e criando um par de partículas reais. Assim, a inflação oferece um mecanismo em que é possível criar matéria do vácuo. Isso faz com que essa teoria seja muito especial, sendo capaz de combinar a mecânica quântica com a relatividade geral.

Dessa forma, a inflação é responsável pela origem das perturbações iniciais. Essas perturbações do campo quântico escalar são geradas em todos os instantes da inflação e com diferentes cumprimentos de onda. Elas são geradas e se "esticam" (seu comprimento de onda cresce) durante a inflação. As perturbações que atingem comprimentos de onda maiores que o raio de Hubble do universo permanecem congeladas até o momento em que o universo se expanda o suficiente e englobe-as em seu horizonte causal. Com isso, todas as perturbações, no fim da inflação, têm essencialmente a mesma amplitude. Podemos, de maneira análoga, pensar que a probabilidade de criação de partículas na inflação é praticamente a mesma em todos os pontos do universo. Como a expansão acelerada é quase constante em todo o espaço, a quantidade de partículas criadas por unidade de volume e por unidade de tempo será também constante. Assim, o padrão dessas perturbações de densidade deve manter-se ao longo da inflação e, com isso, elas têm quase a mesma amplitude em quaisquer escalas. Esse espectro invariante de escala pode ser medido na RCF e é uma importante verificação para esse mecanismo de geração de perturbações e para a teoria da inflação [24].

Ao fim da inflação, quando o campo escalar termina de rolar lentamente em direção ao mínimo de seu potencial efetivo, ele passa a oscilar rapidamente em torno desse mínimo. Conforme vai oscilando, o campo, se estiver acoplado a outros campos de matéria, perde energia por meio da criação de partículas, até atingir o mínimo do potencial. Quase toda a matéria que constitui o universo é criada durante essa fase, chamada de reaquecimento. Esse nome é dado porque todas essas partículas criadas interagem entre si e atingem um estado de equilíbrio térmico com temperatura final $T_{\text {reaq }}$. Esse mecanismo de criação de partículas somente é eficiente, ou seja, cria uma quantidade grande de partículas, quando temos o regime de ressonância paramétrica, quando a amplitude das oscilações do campo em torno do mínimo são amplas. Esse estágio de decaimento rápido e não perturbativo é chamado de pré-aquecimento.

Esses dois mecanismo de criação de partículas no universo primordial são tratados pela Teoria Quântica de Campos em espaços curvos, em que temos um campo livre em um fundo dependente do tempo, ou seja, uma métrica dependente do tempo. Contudo, a criação de partículas não fica bem definida nesse quadro, uma vez que em espaços-tempo curvos os conceitos de vácuo e partículas não estão bem definidos. Dessa forma, convém pensar nesse mecanismo de outra maneira. Se tivermos um campo quântico interagindo com um potencial externo dependente do tempo, ou seja, o potencial gravitacional responsável pela expansão do universo, temos que o mecanismo de criação de partículas pode ser mais claramente estudado. Vamos mostrar nessa dissertação que podemos escrever de 
maneira totalmente equivalente a criação de partículas cosmológica como um problema de um campo interagindo com um potencial externo.

Em eletrodinâmica existe um fenômeno de criação de partículas pela interação de um campo quântico com um campo externo: o efeito Schwinger. O objetivo dessa dissertação é mostrar que a criação de partículas cosmológica pode ser escrita da mesma forma que o efeito Schwinger. Isso é importante pois, como não é possível testarmos esses mecanismos de criação cosmológicos, uma vez que não podemos criar um novo universo que passe por uma fase inflacionária, se mostrarmos que esses mecanismos são análogos aos de criação de partículas da eletrodinâmica, a verificação experimental em Terra do efeito Schwinger seria uma corroboração do mecanismo cosmológico análogo.

O efeito Schwinger consiste no fenômeno de criação de partículas por campos externos elétricos estáticos e oscilatórios. Esse é um efeito não-perturbativo e muito importante da eletrodinâmica quântica (QED) e ocorre devido à instabilidade do vácuo eletromagnético que pode ser polarizado pela sua interação com um campo externo. Julian Schwinger, em 1951 [72], baseado nos trabalhos seminais de fenômenos de polarização do vácuo de Sauter [69], Euler e Heisenber [70] e Weisskopf [71], fez o primeiro cálculo do efeito Schwinger estático via Lagrangeanas efetivas para um campo elétrico constante, em que se obteve a taxa de criação de partículas assim como seus limites experimentais. Desde então diversos métodos foram desenvolvidos para descrever teoricamente esse efeito, em que podemos destacar o método das transformadas de Bogoliubov, o método do tunelamento [99, 100], o de detectores de partículas [101, 102], o método do funcional de onda [103, 104], entre outros. O efeito Schwinger utiliza campos somente elétricos, uma vez que campos magnéticos não produzem partículas[87].

Esse efeito ainda não foi detectado experimentalmente. Sua detecção é de grande relevância, uma vez que seria a primeira validação de um efeito não-perturbativo da QED [75, 76, 77], que já é fortemente comprovada no regime perturbativo. Contudo, a criação de partículas por esse efeito exige campos elétricos muito fortes e não realizáveis com a tecnologia experimental atual. Por isso foi idealizado, nos anos 1980, o análogo oscilatório desse efeito para campos externos oscilatórios, dado que esse tipo de campo é mais fácil de ser realizado experimentalmente [74].

O efeito Schwinger dinâmico consiste na criação de partículas por um campo elétrico dependente do tempo. Esse efeito foi estudado para diversas formas do campo externo aplicado, sendo que apresenta solução analítica para o caso do potencial de Sauter [123]. Vamos estudar nessa dissertação o efeito Schwinger oscilatório, em que o potencial externo é oscilatório. Esse caso é de maior interesse, uma vez que, para sua verificação experimental, campos eletromagnéticos dessa forma são mais facilmente produzidos, além de permitirem uma analogia com sistemas gravitacionais de criação de partículas. Esse efeito foi estudado teoricamente, também, por diversos formalismos.

Tentativas de detecção do efeito Schwinger oscilatório ainda não foram bem sucedidas; 
os limites experimentais para sua detecção ainda não foram alcançados. Contudo, existe um efeito quântico, que consiste em uma generalização do efeito Casimir, este sim próximo de ser detectado: o efeito Casimir dinâmico, que consiste na produção de fótons pelo movimento acelerado da(s) parede(s) de uma cavidade ${ }^{1}$ no vácuo. O experimento MIR, em construção e testes, apresenta-se como uma possível detecção para o efeito Casimir dinâmico e de efeitos não-perturbativos do vácuo quântico. Resultados são esperados para 2009.

O propósito dessa dissertação é fazer uma analogia entre a criação de partículas na inflação e no pré-aquecimento com o efeito Schwinger. Ambos os mecanismos consistem em osciladores paramétricos com freqüência dependente do tempo, mudando apenas a natureza do campo externo aplicado. Propomo-nos a mostrar que a criação de partículas na inflação pode ser descrita, a menos de uma transformação canônica, pelas mesmas equações que descrevem a criação de partículas para o efeito Schwinger estático, sendo assim equivalentes. No caso do pré-aquecimento, mostraremos que a criação de partículas pode ser descrita por uma equação de Mathieu, assim como no efeito Schwinger oscilatório. Assim, com a medição do efeito Schwinger podemos confirmar que esse efeito não-perturbativo de criação de partículas do vácuo é realizável e com isso possível de ter acontecido no universo primordial.

Como o tema central de investigação na dissertação é a comparação entre os efeitos Schwinger estático e oscilatório com a criação de partículas na inflação e no préaquecimento, demostrando que esses dois fenômenos são descritos basicamente pela mesma teoria, essa verificação central será feita no contexto do formalismo das transformadas de Bogoliubov. Na dissertação descrevemos detalhadamente o formalismo geral do efeito Schwinger estático, que nos permitirá a comparação com o formalismo que descreve a teoria da inflação. A criação de partículas é descrita via três formalismos: o da teoria do espalhamento, via a teoria de transformadas de Bogoliubov e via estados squeezed. A descrição via teoria de espalhamento, apesar de ser apresentada de forma detalhada, serve-nos simplesmente para a obtenção dos limites experimentais de observação do efeito. O tema central do capítulo do efeito Schwinger estático é, portanto, o formalismo via transformadas de Bogoliubov e via estados squeezed.

Para o efeito Schwinger oscilatório, de maneira análoga ao caso estático, temos o efeito descrito via três formalismos. Novamente, apesar de sua descrição extensa e detalhada, o cálculo via teoria de espalhamento fará com que obtenhamos os limites observacionais desse efeito. Eles serão considerados na análise do caso central de interesse, que é a descrição via transformadas de Bogoliubov. No caso oscilatório, o formalismo utilizado para computar esse efeito foi aplicado aqui pela primeira vez para esse tipo de sistema, e

\footnotetext{
${ }^{1}$ Não necessariamente uma cavidade. Esse efeito ocorre também em placas paralelas no vácuo, em uma única placa no vácuo, entre outras configurações.
} 
baseia-se na análise feita para a criação de partículas por ressonância paramétrica no préaquecimento. No capítulo do efeito oscilatório apresentamos, também, o efeito Casimir dinâmico, análogo dinâmico do conhecido efeito Casimir, no qual a oscilação da placas do capacitor que estão colocado no vácuo faz o papel do campo externo aplicado [136, 79, 80]. Esse efeito é apresentado por ser um efeito análogo ao Schwinger oscilatório, mas principalmente porque, pela ausência de experimentos de detecção próxima do efeito Schwinger, é ele que está mais próximo de ser detectado. Novamente, a descrição desse efeito é detalhada e extensa, mas o importante a ser levado em conta é a verificação de que pode ser entendido como um efeito análogo ao Schwinger oscilatório e leva-nos, ao final, a um número de partículas criadas que possibilita a detecção experimental.

Organizamos a dissertação da seguinte maneira. No capítulo 2 daremos uma introdução do modelo cosmológico padrão, identificando os aspectos da estrutura, expansão e evolução do universo. Identificaremos também os problemas que limitam esse modelo. Com isso, partiremos para o Capítulo 3, em que desenvolvemos a teoria da inflação e do pré-aquecimento e reaquecimento. Mostraremos como essa teoria resolve os problemas do modelo do Big Bang, sua teoria geral e seu período final em que quase todas as partículas elementares do universo foram criadas, o reaquecimento. Em seguida, na Capítulo 4, descreveremos a teoria das perturbações cosmológicas, analisando tanto sua evolução como seu surgimento devido às flutuações quânticas na inflação. Partiremos então para a descrição do efeito Schwinger estático, no Capítulo 5 e oscilatório no Capítulo 6. No Capítulo 5, daremos uma introdução teoria quântica de campos na presença de campos externos e descreveremos o efeito Schwinger em três diferentes formalismos. No Capítulo 6, descreveremos a criação de partículas por um campo externo oscilatório, determinando também seus limites observacionais. Com isso, introduziremos o efeito Casimir dinâmico, que representa a mais promissora chance de observação da criação de partículas do vácuo por um campo externo. Em seguida, no Capítulo 7, será descrito o tema central dessa dissertação, quando faremos a analogia entre os sistemas cosmológicos e eletromagnéticos, mostrando, no fim, que são equivalentes. Concluímos o trabalho, então, no Capítulo 8. 


\section{Capítulo 2}

\section{Modelo Cosmológico Padrão}

O Modelo Cosmológico Padrão (MCP) (ou Teoria do Big Bang Quente) é a base para o nosso atual entendimento e representação do universo, descrevendo sua evolução a partir de um estado incial de grande densidade e temperatura há aproximadamente 13,9 bilhões de anos e que vem se expandindo e esfriando desde então. Nesse modelo temos um universo homogêneo e isotrópico, aproximadamente plano, composto principamente por matéria bariônica $(\sim 4 \%)$, radiação eletromagnética $(\sim 0,001 \%)$, um tipo de matéria que não sabemos a origem, denominada matéria escura $(\sim 23 \%)$ e uma energia também de origem desconhecida, chamada energia escura $(\sim 73 \%)[22,23,24,25]$. Esse modelo é amplamente aceito e coleciona uma grande quantidade de sucessos teóricos e observacionais. Apesar de apresentar alguns problemas e necessitar de teorias complementares, ainda é o modelo adotado para descrever a estrutura, expansão e evolução do universo.

O MCP baseia-se em dois pilares teóricos: a teoria da relatividade geral, que supomos descrever a dinâmica do universo como um todo, e o princípio cosmológico, hipótese simplificadora que afirma que o universo é espacialmente homogêneo e isotrópico. Além disso, ele se apoia em três pilares observacionais: a lei de Hubble, que mostra que o universo está em expansão; a abundância dos elementos leves, que confirma as hipóteses da nucleossíntese primordial; e a radiação cósmica de fundo $(\mathrm{RCF})$, uma radiação isotrópica, de corpo negro, com temperatura de aproximadamente $2,75 \mathrm{~K}$.

O MCP descreve com boa precisão os aspectos geométricos do universo, definindo sua estrutura e sua evolução dinâmica, mas também prevê de maneira muito precisa a abundância de seus componentes materiais. Mostraremos nesse capítulo primeiramente as previsões geométricas do modelo, expondo os conceitos e equações básicas que emergem dessa teoria e introduzindo seus parâmetros cosmológicos de relevância e suas relações. Podemos assim descrever o MCP de maneira geral, apresentando também suas previsões sobre a abundância dos elementos químicos e descrevendo a história térmica do universo. Após definirmos o modelo, apresentaremos seus pilares observacionais e principais problemas da teoria. 


\subsection{Previsões geométricas do MCP}

Os principais resultados que emergem do MCP vêm do estudo de suas características cinemáticas e dinâmicas. Essa descrição se apoia em hipóteses simplificadoras, posteriormente testadas, e na teoria da relatividade geral, que rege sua dinâmica. Dividiremos o estudo dessas previsões em três partes: o estudo de sua estrutura, expansão e evolução.

\subsubsection{Estrutura do universo}

O MCP nos diz que a dinâmica do universo é descrita pelas equações de Einstein. Logo no primeiro modelo cosmológico introduzido por Einstein em 1917, notou-se que essas equações envolvem dez equações não-lineares muito difíceis de serem resolvidas. Assim, para podermos achar uma solução, é necessário impor-se algumas condições extras ou simetrias adicionais ao problema. Para resolver esse problema, foi imposto o princípio cosmológico, que nos diz que o universo deve ser espacialmente homogêneo e isotrópico em largas escalas ${ }^{1}$.

Por homogeneidade entendemos que todos os pontos espaciais são equivalentes, ou seja, uma invariância por translação. Por isotropia em torno de um ponto entendemos que todas as direções a partir de um ponto são equivalentes, ou seja, invariância por rotações. Assim, por homogeneidade e isotropia, podemos entender que todas as posições no universo são equivalentes, vale dizer, aqui na Terra ou em qualquer outro ponto do universo iremos verificar a mesma expansão do universo. Isso significa que a Terra não está no centro do universo ou melhor, que o universo não tem um centro, uma vez que não existe uma posição privilegiada no universo.

Essa hipótese é bem testada e observações atuais do catálogo de galáxias do Sloan Digital Sky Survey [26, 27] verificam que o universo é sensivelmente o mesmo em todos os sentidos e direções em escalas superiores a $100 \mathrm{Mpc}$ (na verdade de 100 a $1000 \mathrm{Mpc}$ ). Isso também é confirmado pelas observações de RCF, que apresenta a mesma temperatura, exceto por pequenos desvios, para todas as regiões do universo observável. O princípio cosmológico é válido apenas em escalas cosmológicas, sendo que em menores escalas o universo é altamente inomogêneo, como podemos ver com a presença de galáxias, aglomerados etc.

O princípio cosmológico trata essencialmente da parte espacial do cosmos. Contudo, como vivemos em um espaço-tempo quadridimensional, falta definirmos as propriedades da coordenada temporal. Na relatividade geral, que é a teoria que descreve o nosso universo, não há referenciais preferenciais e, portanto, também não há um tempo absoluto. Então como descrever o universo com essa teoria, uma vez que o universo parecerá

\footnotetext{
${ }^{1}$ Einstein em seu primeiro modelo cosmológico impôs a versão original do princípio cosmológico em que além da homogeneidade e isotropia, o universo também deveria ser estático. Essa hipótese adicional levou à introdução de uma constante cosmológica em suas equações, o que, após as descobertas de Hubble de que o universo está em expansão, foi descrita por ele como seu maior erro.
} 
diferente para observadores em diferentes estados de movimento? Para resolver esse problema Weyl [28], em 1923, acrescentou outra hipótese simplificadora para o universo, um corolário do princípio cosmológico. Weyl postulou que existe uma classe privilegiada de observadores que estão associados ao movimento coletivo das galáxias com a expansão do universo, excluídos quaisquer movimentos relativos dessas. Esse observador é chamado de observador comóvel, pois ele se move junto com a expansão do universo. Dessa forma, esses observadores encontram-se em repouso em relação a um substrato que preenche todo o universo e que pode ser definido como sendo um fluido. Assim, temos o postulado de Weyl que define que as partículas desse substrato situam-se na congruência de geodésicas ${ }^{2}$ do tipo tempo divergindo de um ponto no infinito passado ou no infinito futuro. Além disso, essas geodésicas não devem se interceptar e somente uma passa por cada ponto do espaço-tempo. Com isso, podemos entender que o substrato, na verdade, pode ser interpretado como um fluido perfeito. Esse é o resultado mais importante desse postulado. Podemos ainda enxergar o universo como (hiper)superfícies espaciais, homogêneas e isotrópicas, definidas para cada tempo, chamado de tempo próprio.

Uma vez que, de acordo com a teoria da relatividade geral, a geometria do espaçotempo é determinada pelo seu conteúdo material e este está distribuído de uma maneira homogênea e isotrópica, a geometria espacial do universo deve ser escrita por uma métrica que seja globalmente homogênea e isotrópica. A única métrica que oferece soluções exatas das equações de Einstein, imposto o princípio cosmológico, é a métrica de FriedmannRobertson-Walker (FRW ou FLRW - Friedmann-Lemaître-Robertson-Walker), denominada assim em homenagem a A. Friedmann que em 1922-1924 a derivou e a H. P. Robertson e A. G. Walker, que provaram que ela é única. Essa métrica, como não há direções privilegiadas no universo, apresenta somente dependência temporal e é definida pelo elemento de linha:

$$
\begin{aligned}
d s^{2} & =d t^{2}-a^{2}(t) \underbrace{\left[\frac{d r^{2}}{1-k r^{2}}+r^{2}\left(d \theta^{2}+\operatorname{sen}^{2} \theta d \varphi^{2}\right)\right]}_{d l^{2}} \\
& =g_{\mu \nu} d x^{\mu} d x^{\nu} .
\end{aligned}
$$

O elemento $d l$ representa a distância entre dois observadores comóveis, uma vez que eles movem-se com o fluido que compõe o universo e que, para eles, é homogêneo e isotrópico. Podemos ver isso se considerarmos o movimento de um fóton que tem deslocamento nulo no espaço-tempo, ou seja, $d s^{2}=0$ (geodésica). Com isso, de (2.1):

$$
c d t=d x_{f i s}=a(t) d l
$$

\footnotetext{
${ }^{2}$ Geodésicas são o menor caminho que se pode percorrer no espaço-tempo. Vamos definir a geodésica a seguir quando falarmos da propagação de um fóton, que sempre anda seguindo uma geodésica.
} 
em que $d x_{f i s}$ representa a distância física que o fóton percorreu e a(t) é chamado de fator de escala cósmico e contém informação sobre dinâmica do universo homogêneo e isotrópico. Seu valor atualmente é $a\left(t_{0}\right)=1$, por convenção. Assim, a distância física depende do fator de escala, uma vez que $d l$ é constante. Logo, $d l$ é chamado de distância comóvel e as coordenadas $(r, \theta, \varphi)$ são chamadas de coordenadas esféricas comóveis, uma vez que para elas a expansão é homogênea e isotrópica. A coordenada temporal em (2.1) é chamada de tempo cosmológico ou tempo próprio e representa o tempo medido por observadores comóveis. É usual em cosmologia utilizar também outra variável temporal adimensional, o tempo conforme, definido pela parametrização $d \eta=d t / a(t)$.

Podemos também escrever a métrica na forma mais compacta $(2.2)$, em que $x^{\mu}=$ $(t, r, \theta, \varphi)$ e $g_{\mu \nu}$ é o tensor métrico que, no caso da métrica de FRW, é representado pela matriz diagonal:

$$
g_{\mu \nu}=\left(\begin{array}{cccc}
-1 & 0 & 0 & 0 \\
0 & \frac{a^{2}(t)}{1-k r^{2}} & 0 & 0 \\
0 & 0 & a^{2}(t) r^{2} & 0 \\
0 & 0 & 0 & a^{2}(t) r^{2} \operatorname{sen}^{2} \theta
\end{array}\right) .
$$

A métrica acima descreve a geometria do universo se, e somente se, seu conteúdo material está distribuído de maneira homogênea e isotrópica. Assim, imposto o princípio cosmológico, isso implica que teremos uma curvatura constante em cada ponto do universo. Existem somente três tipos de espaços tridimensionais com topologias triviais que satisfazem essa condição [31]: o espaço plano $\mathbb{E}^{3}$, a esfera tridimensional $\mathbb{S}^{3}$ e um espaço hiperbólico tridimensional $\mathbb{S}^{2,1}$. A métrica de FRW contempla esses três espaços que são determinados pelo valor da constante de curvatura $k$ no elemento de linha:

- Para $k=0$, temos $\mathbb{E}^{3}$, que representa uma solução com universo plano;

- Para $k=+1$, temos $\mathbb{S}^{3}$, que representa um universo fechado com volume finito dado por $V_{\mathbb{S}^{3}}=4 \pi a^{2}(t)$.

- Para $k=-1$, temos $\mathbb{S}^{2,1}$, que representa um universo aberto, com volume infinito e raio de curvatura imaginário.

No caso do universo plano, podemos ver que se o fator de escala é uma constante ou varia muito lentamente $[\dot{a}(t) \approx 0]$, a métrica (2.1) descreve um espaço de Minkowski.

\subsubsection{Expansão do universo}

A verificação de que o universo está se expandindo constituiu uma das maiores descobertas do século XX, uma vez que representa uma quebra no paradigma vindo desde a 
antiguidade e considerado até por Einstein, de que o universo é estático. Devido à infinidade de soluções para as equações de Einstein, inclusive a estática imposta por ele mesmo, era necessária alguma prova de que o universo não fosse estático e as soluções dinâmicas dessas equações fossem aceitas como as verdadeiras. A lei de Hubble é uma lei empírica descoberta em 1929 [3] pelo astrônomo Edwin Hubble durante a investigação de cefeidas, que são estrelas variáveis, fora da Via Láctea. Hubble verificou que todas as galáxias estavam se afastando de nós, obtendo a relação linear:

$$
v=H_{0} l
$$

em que $v$ é a velocidade de recessão das galáxias, $l$ é sua distância até a Terra e $H_{0}$ é chamado de parâmetro de Hubble. Vimos anteriormente que a expansão do universo define dois tipos diferentes de coordenadas, que são as coordenadas físicas e as comóveis, que se relacionam por (2.3). Assim, é possível obtermos a lei de Hubble através dessa relação, derivando a expressão em relação ao tempo próprio, e ignorando quaisquer velocidades intrínsecas dos objetos, obtendo:

$$
\begin{aligned}
v & =\frac{\dot{a}(t)}{a(t)} l \\
& =H(t) l,
\end{aligned}
$$

em que $H(t)$ é a função de Hubble, sendo que a melhor estimativa para seu valor atual [24] é:

$$
H_{0}=70,5 \pm 1,3 \mathrm{~km} \mathrm{~s}^{-1} \mathrm{Mpc}^{-1}
$$

A determinação desse parâmetro é feita através dos dados do satélite WMAP combinado com as medidas de distância das supernovas do tipo Ia e a Oscilação Acústica dos Bárions(BAO) na distribuição das galáxias.

As observações de Hubble da recessão das galáxias foram feitas observando que a luz emitida por elas tinha um desvio no seu comprimento de onda para a região do vermelho do espectro eletromagnético, conhecido como redshift. A velocidade de recessão pode ser inferida através do redshift, uma vez que esse é interpretado como um efeito Doppler cosmológico. Podemos ver isso se pensarmos na emissão de um onda eletromagnética em um instante $t_{e m}$ por uma fonte, como por exemplo uma galáxia, localizada nas coordenadas comóveis $\left(r_{e m}, \theta_{e m}, \varphi_{e m}\right)$. A propagação dessa onda - fóton - obedece à geodésica nula $d s^{2}=0$ e a distância percorrida (chamamos de distância, uma vez que temos no lado esquerdo da equação $c d t$, mas $c=1$, no sistema de coordenadas natural que estamos utilizando) para chegar até nós é dada por: 


$$
\int_{t_{e m}}^{t_{o b s}=t_{0}} \frac{d t^{\prime}}{a\left(t^{\prime}\right)}=\int_{r_{e m}}^{r_{o b s}=r_{0}=0} \frac{d r^{\prime}}{\sqrt{1-k r^{\prime 2}}} .
$$

Essa expressão é independente do tempo, uma vez que as coordenadas comóveis são constantes. Logo, se a onda fosse emitida em um instante posterior $t_{e m}+\delta t_{e m}$, chegando aqui em $t_{0}+\delta t_{0}$, o resultado de (2.8) seria então o mesmo. Assim, substraindo a expressão para tempos diferentes temos que:

$$
\frac{\delta t_{e m}}{a\left(t_{e m}\right)}=\frac{\delta t_{o b s}}{a\left(t_{o b s}\right)} .
$$

Podemos reescrever essa relação em função da frequência, uma vez que $\nu=1 / t$, e do comprimento de onda, $\nu=\lambda^{-1}$ no sistema de coordenadas natural, obtendo a relação:

$$
\frac{\nu_{o b s}}{\nu_{e m}}=\frac{a\left(t_{e m}\right)}{a\left(t_{o b s}\right)}=\frac{\lambda_{e m}}{\lambda_{o b s}}
$$

Com essa expressão podemos ver os efeitos da expansão do universo sem nem mesmo conhecer a lei que a rege, uma vez que a expansão faz com que o fator de escala aumente, consequentemente a frequência da onda diminui e seu comprimento de onda aumenta indo em direção aos comprimentos de onda do vermelho no espectro da radiação eletromagnética. Temos, assim, um desvio para o vermelho, ou redshift. Esse resultado é importante também, uma vez que a RCF tem uma distribuição Planckiana de seu espectro que se preserva na expansão do universo.

Convenientemente podemos expressar a relação (2.10) em termos do fator de redshift:

$$
z=\frac{\lambda_{o b s}-\lambda_{e m}}{\lambda_{e m}}=\frac{a\left(t_{o b s}\right)}{a\left(t_{e m}\right)}-1 .
$$

Assim, dizemos que se $z>0$ temos um redshift e se $z<0$ temos um blueshift. Podemos relacionar esse parâmetro com a velocidade de recessão se pensarmos que uma galáxia relativamente próxima de nós se moverá para longe com velocidade dada pela lei de Hubble (2.6). Expandindo (2.11) em série de Taylor podemos ver que $z \simeq\left(t_{o b s}-t_{e m}\right) H\left(t_{0}\right) \simeq$ $H\left(t_{0}\right) l=v$, se a distância da galáxia não é muito grande. Logo, a medida do parâmetro de redshift nos fornece uma relação com a velocidade de recessão.

A partir da constante de Hubble, podemos derivar diversos parâmetros cinemáticos do nosso universo, como o tempo de Hubble e o raio de Hubble. Esses parâmetros são somente aproximações, mas nos permitem ter uma idéia da ordem de grandeza desses observáveis.

Pela lei de Hubble, podemos verificar que, como (aproximadamente) $v / l=t$, então a constante de Hubble tem ordem de grandeza do inverso do tempo. Assim, define-se o tempo de Hubble como sendo: 


$$
t_{H} \equiv H^{-1}
$$

Analogamente a essa definição, podemos calcular o raio de Hubble, sendo ele:

$$
R_{H}=c t_{H}=c H^{-1}
$$

Utilizando (2.7), que é o valor mais aceito do parâmetro de Hubble, vemos que o tempo de Hubble é dado por $t_{H}=14 \times 10^{9}$ anos e o raio de Hubble por $R_{H} \approx 4,27 \times 10^{6} \mathrm{Mpc}$.

\subsubsection{Evolução do universo}

A descrição cinemática do universo feita anteriormente é incompleta, uma vez que não fornece nenhuma predição sobre o fator de escala, a única componente dinâmica apresentada. Para podermos definir o comportamento do fator de escala é necessário estudar o conteúdo material do universo. A dinâmica e também a cinemática do universo são regidas pela teoria da relatividade geral de Einstein, que com suas equações de campo, válidas em todos os pontos do universo, nos dizem como o conteúdo material (energético) do universo afeta sua geometria. As equações de Einstein são escritas na forma [30, 31, 29]:

$$
G_{\mu \nu} \equiv R_{\mu \nu}-\frac{1}{2} g_{\mu \nu} R=8 \pi G T_{\mu \nu}
$$

em que o lado esquerdo contém as informações sobre a geometria, sendo que $G_{\mu \nu}$ é chamado de tensor de Einstein, que expressa a curvatura do espaço tempo através de suas componentes $R_{\mu \nu}$ que representa o tensor de Ricci e $R$ é o escalar de Ricci ou de curvatura. Já o lado direito da equação representa o conteúdo material do universo, sendo que $T_{\mu \nu}$ é o tensor de energia-momentum (TEM). Originalmente essas equações também continham o termo $-\Lambda \delta_{\mu \nu}$, em que $\Lambda$ é a constante cosmológica. Esse termo foi colocado por Einsten "à mão" para fazer com que o universo fosse estático. Apesar de Einstein ter considerado esse seu maior erro, após a confirmação da expansão do universo por Hubble, a constante cosmológica voltou para a cena da cosmologia com a descoberta de que o universo está em expansão acelerada $[12,13,14]$ e essa aceleração poderia ser causada por ela.

Mas somente as equações de Einstein não são suficientes para chegarmos em equações para a descrição do nosso universo. A teoria da relatividade geral não consegue definir características para a forma do tensor de energia-momento. Supusemos anteriormente, pelo princípio cosmológico, que a matéria no universo está distribuída de forma homogênea e isotrópica e, pelo postulado de Weyl, que o universo pode ser considerado, em boa aproximação, como preenchido por um fluido perfeito, com TEM dado por:

$$
T_{\mu \nu}=(\rho+p) u_{\mu} u_{\nu}-p g_{\mu \nu}
$$


em que $\rho$ é a densidade de energia do fluido, $p$ sua pressão e $u_{\mu}$ é o quadrivetor de velocidade do fluido. Em um sistema de coordenadas comóveis a quadrivelocidade é constante dada por $u_{\mu}=(1,0,0,0)$ e assim o TEM assume a forma $T_{\nu}^{\mu}=\operatorname{diag}(\rho,-p,-p,-p)$. Como esse fluido é homogêneo e isotrópico espacialmente, então $\rho$ e p dependem somente do tempo.

Podemos considerar também que o universo é preenchido por um tipo diferente de matéria (ou campo de matéria): o campo escalar. Para descrever esse campo, temos que um TEM da forma:

$$
T_{\nu}^{\mu}=\varphi^{, \mu} \varphi_{, \nu}-\left[\frac{1}{2} \varphi^{, \gamma} \varphi_{, \gamma}-V(\varphi)\right] \delta_{\nu}^{\mu}
$$

que também pode ser escrito como o TEM do fluido perfeito se definirmos:

$$
\begin{aligned}
\rho & \equiv \frac{1}{2} \varphi^{, \gamma} \varphi_{, \gamma}+V(\varphi), \\
p & \equiv \frac{1}{2} \varphi^{, \gamma} \varphi_{, \gamma}-V(\varphi), \\
u^{\alpha} & \equiv \varphi^{, \alpha} / \sqrt{\varphi^{, \gamma} \varphi_{, \gamma}},
\end{aligned}
$$

em que $\varphi^{, \gamma} \varphi_{, \gamma}>0$

Precisamos determinar as funções $a(t)$ e $\rho(t)$ para determinarmos a dinâmica do universo. Através do TEM do fluido, inserimos (2.1) e (2.15) nas equações de Einstein (2.14), que então se reduzem às equações de Friedmann (ou Friedmann-Lemaître):

$$
\begin{aligned}
\left(\frac{\dot{a}}{a}\right)^{2} & =H^{2}=\frac{8 \pi G}{3} \rho-\frac{k}{a^{2}}, \\
\frac{\ddot{a}}{a} & =\dot{H}+H^{2}=-\frac{4 \pi G}{3}(\rho+3 p),
\end{aligned}
$$

em que $\rho$ e $p$ são a soma das densidades de energia e pressão de todas as diferentes componentes materiais do universo. Podemos combinar essas equações tomando a derivada temporal (2.20) e usando (2.21), obtendo:

$$
\dot{\rho}+3 H(\rho+p)=0,
$$

que é chamada de equação da continuidade. Essa equação expressa a conservação do TEM e pode ser obtida alternativamente através de $T_{\nu ; \mu}^{\mu}=0$. No caso do campo de matéria escalar, essa conservação é expressa pela equação

$$
\varphi_{; \mu}^{; \mu}+\frac{\partial V(\varphi)}{\partial \varphi}=0
$$


que é a conhecida equação de Klein Gordon, ou seja, a equação de movimento para o campo escalar.

A equação (2.22) é também chamada de "condição de adiabaticidade". Podemos ver isso através da primeira lei da termodinâmica:

$$
d E=T d S-p d V
$$

em que $E$ é a energia total, $T$ é a temperatura, $S$ a entropia e $V$ o volume. Como $\rho=E / V$, ela assume a forma:

$$
d \rho=T d S-(\rho+p) \frac{d V}{V} .
$$

Sabendo que o volume do universo é da ordem $V \propto a^{3}$, assim $d V / V=3 d a / a$. Substituindo isso na equação da continuidade (2.22), obtemos que $\dot{S}=0$, ou seja, a entropia do universo é constante. Podemos ver que a expansão do universo, regida pelas equações de Friedmann, é adiabática.

A partir das equações de Friedmann também podemos inferir algumas características cinemáticas do universo. Como já mencionamos, os parâmetros $\rho$ e $p$ são, na verdade, a soma de suas componentes para diferentes tipos de matéria. Dessa forma, se dividirmos os dois lados da equação (2.20) por $H^{2}$, podemos reescrevê-la da forma:

$$
1=\Omega_{t o t a l}-\frac{k}{a^{2} H^{2}}
$$

em que $\Omega_{\text {total }}=\sum_{i} \Omega_{i}$ e $\Omega_{i} \equiv \rho_{i} / \rho_{\text {crit }}$ são as densidades relativas de cada componente material (energético). O parâmetro:

$$
\rho_{\text {crit }}=\frac{3 H^{2}}{8 \pi G},
$$

é chamado de densidade crítica. Seu valor mais aceito atualmente é $\rho_{\text {crit }}=1,88 h^{2} \times$ $10^{-29} \mathrm{~g} \mathrm{~cm}^{-3}=8,098 \mathrm{~h}^{2} \times 10^{-11} \mathrm{eV}^{4}$, considerando o parâmetro de Hubble , $H_{0}$ citado acima. De (2.26) podemos ver que o parâmetro de densidade total pode ser escrito em função da constante de curvatura, $\Omega_{\text {total }}=1+k / a^{2} H^{2}$, relacionando, assim, o conteúdo material do universo com a curvatura, como prevê a relatividade geral. Assim, podemos ver que se:

$$
\begin{array}{ll}
\Omega_{\text {total }}>1 \Leftrightarrow k=+1, & \text { Universo fechado } \\
\Omega_{\text {total }}=1 \Leftrightarrow k=0, & \text { Universo plano } \\
\Omega_{\text {total }}<1 \Leftrightarrow k=-1, & \text { Universo aberto. }
\end{array}
$$

Observações atuais feitas pelos experimentos WMAP e BOOMERanG, mostram que o 
valor atual de $\Omega_{\text {total }}$ é da ordem de $1,02 \pm 0,02$ [15, 24], ou seja, muito próximo da unidade, que descreve um universo plano. Contudo, no MCP não há mecanismos capazes de explicar a preferência por essa planura do universo. Descreveremos na Seção 2.4 melhor esse problema.

Podemos observar facilmente que o universo é constituído de diferentes componentes de matéria. Para descrever eeses constituintes, as componentes da densidade total podem ser divididas em $\Omega_{\text {total }}=\Omega_{m}+\Omega_{\text {rad }}+\Omega_{\Lambda}$, em que $\Omega_{m}$ é a componente de matéria que podemos dividir em matéria bariônica, $\Omega_{b}$, e escura, $\Omega_{d m} ; \Omega_{r a d}$ é a componente de radiação; e $\Omega_{\Lambda}$ a componente de constante cosmológica ou energia escura. Os valores da fração de cada uma dessas densidades podem ser inferidos observacionalmente [24].

As equações de Friedmann (e da continuidade) são um sistema de equações que nos fornecem a dinâmica de $a(t), \rho(t)$ e $p(t)$. Contudo, para sua total determinação é necessário um sistema com três equações linearmente independentes umas das outras. Como a equação da continuidade é derivada das equações de Friedmann, necessitamos de mais uma equação para determinar nosso sistema. Uma vez que todas as simetrias referentes ao espaço-tempo e às equações de Einstein já foram utilizadas, vamos recorrer a uma característica do fluido que utilizamos para descrever o universo que relaciona a densidade de energia e a pressão através de uma equação de estado da forma:

$$
p=\omega \rho .
$$

Essa equação é útil também, para discriminar entre cada constituinte do universo nas equações que descrevem sua dinâmica. Uma vez que são esses constituintes os responsáveis pela dinâmica, cada um deles influencia de maneira diferente a evolução do universo. Cada um desses constituintes é descrito por um certo valor do parâmetro $\omega$ que é, em geral, assumido constante. Para os três fluidos do universo temos que $\omega=0$ para a matéria (bariônica e escura), $\omega=1 / 3$ para a radiação e $\omega=-1$ para a constante cosmológica.

Assim, com o uso da equação de estado (2.31) no sistema de equação das equações de Friedmann, supondo que os diferentes constituintes não interagem entre si, integramos (2.22) obtendo:

$$
\rho(a)=\rho_{0} a(t)^{-3(1+\omega)}
$$

em que $\rho_{0}$ é o valor atual da densidade de energia. A partir dessa equação podemos ver o comportamento para os três fluidos do universo:

- Matéria: Para a matéria (bariônica e escura) não relativística, com equação de estado $p=0$, temos que a densidade de energia evolui da forma: $\rho(a)=\rho_{0}^{m} a^{-3}$. Podemos ver que essa densidade é inversamente proporcional ao volume físico do universo. 
- Constante Cosmológica: Com equação de estado $p=-\rho$, a densidade de energia da constante cosmológica é constante, $\rho(a)=\rho_{0}^{\Lambda}$, ou seja, não muda com a evolução do universo. No infinito futuro, essa densidade será dominante em relação às outras.

- Radiação: No caso da radiação, com equação de estado $p=\rho / 3$, temos que $\rho(a)=$ $\rho_{0}^{r a d} a^{-4}$. Dessa expressão podemos concluir que a densidade de energia da radiação sempre irá dominar sobre as outras no infinito passado. A partir desse resultado é possível verificar o que vimos anteriormente, que a radiação sofre redshift, uma vez que $E=h \nu$ e $\nu \propto a^{-1}$. Outro resultado que podemos inferir conhecendo a densidade de energia é que a radiação se relaciona com a temperatura da forma: $\rho=E / V \propto T_{\text {rad }}^{4}$. Dessa forma a temperatura da radiação evolui como $T_{\text {rad }} \propto a^{-1}$.

Finalmente, para determinarmos a variável dinâmica restante, o fator de escala, substituimos a solução (2.32) em (2.20), para o caso em que $k=0$ e $\omega$ é constante, e obtemos:

$$
a(t)=a_{0} t^{2 / 3(1+\omega)} .
$$

Essa solução nos mostra como o conteúdo material do universo altera sua evolução. Podemos mostrar, assim, que em um universo dominado por cada um dos três fluidos apresentados, o fator de escala evolue de acordo com:

$$
a(t)= \begin{cases}a_{0}^{m} t^{2 / 3}, & \text { matéria } \\ a_{0}^{r a d} t^{1 / 2}, & \text { radiação } \\ a_{0}^{\Lambda} e^{H_{\Lambda} t}, & \text { const. cosmol. }\end{cases}
$$

em que $H_{\Lambda}=\sqrt{8 \pi G \rho_{0}^{\Lambda} / 3}$. Dessas soluções podemos ver, pela sua segunda derivada, que para a matéria e para a radiação a expansão do universo é desacelerada, enquanto que para a constante cosmológica ela é acelerada. Esse último caso é chamado de universo de de Sitter.

Voltando ao caso do campo escalar, podemos ver que no caso em que o termo do potencial é dominante, de (2.17), temos que $p=-\rho$. Nesse caso o TEM do campo escalar tem a mesma forma do TEM da constante cosmológica e com isso $\Lambda=8 \pi G V(\varphi)$. Assim, um universo dominado por um campo escalar expandiria exponencialmente, como visto para a constante cosmológica. Falaremos mais sobre esse tipo de expansão no Capítulo 3 onde discutimos o universo inflacionário.

\subsubsection{Horizontes}

Uma vez que a velocidade da luz é constante e o universo está expandindo, existe um limite para o que é acessível para um observador qualquer nesse universo. Esse limite 
do que pode ser observável é conhecido como horizonte. Essa noção pode ser dividida em dois tipos, que definem diferentes limites de observação: o horizonte de partículas e o horizonte de eventos. Esses horizontes são complementares, uma vez que definem o espaço observável do início do universo até o observador num dado instante e do observador até o infinito futuro.

\subsubsection{Horizonte de partículas}

O horizonte de partículas é a distância máxima que uma partícula (de massa zero fóton) pode ter viajado até um observador desde o início do universo. Sendo nós os observadores, esse horizonte representa a porção do universo que podemos observar hoje em dia, nosso universo observável. Supondo que a luz não tenha sofrido nem espalhamento nem absorção, a máxima distância conforme que a luz pode percorrer desde um instante inicial $t_{i}=0$, que representamos como sendo o início do universo, até um observador no instante $t$, é definido como sendo o horizonte de partículas:

$$
\chi_{p}(t)=a(t) \underbrace{\int_{0}^{t} \frac{d t}{a(t)}}_{\eta},
$$

em que $\eta$ é o tempo passado desde o Big Bang até o observador em $t$. Se $\chi_{p} \rightarrow \infty$, não existe horizonte de partículas, portanto $\chi_{p}<\infty$ para que haja horizonte. Uma vez que consideramos o horizonte desde o início do universo $\left(t_{i}=0\right)$ isso define um universo com uma singularidade inicial. Eventos ocorridos fora desse horizonte não estão em contato causal com os eventos dentro do horizonte. Isso quer dizer que qualquer informação de eventos fora do horizonte de partículas de um observador não puderam chegar a ele. Essa falta de contato entre pontos do universo desde seu início é um problema, uma vez que regiões distantes em nosso universo apresentam características muito semelhantes. Falaremos mais desse problema na Seção 2.4.

De (2.34) e (2.35) podemos determinar o horizonte de partículas para os diferentes constituintes. Para um universo dominado por matéria, $\chi_{p}(t)=3 t$ e para um universo dominado por radiação, $\chi_{p}(t)=2 t$.

\subsubsection{Horizonte de eventos}

O horizonte de eventos é definido como sendo a distância que a luz pode percorrer a partir de um observador até o tempo final do universo. Isso define o volume do espaço-tempo contendo eventos que serão observados no futuro. Supondo novamente que a luz não sofra nem espalhamento nem absorção em seu caminho, a distância conforme que a luz pode percorrer de um observador no instante $t$ até o tempo final $t_{\text {final }} \rightarrow \infty$ é dado por: 


$$
\chi_{e}(t)=a(t) \int_{t}^{\infty} \frac{d t}{a(t)},
$$

e chamada de horizonte de eventos. Eventos ocorridos fora desse horizonte nunca serão observados. Esse horizonte somente está presente se $\chi_{e}<\infty$. Para o caso de um universo dominado por matéria ou radiação, não há horizonte de eventos, pois $\chi_{e} \rightarrow \infty$. Já para o caso de um universo dominado por constante cosmológica, esse horizonte de eventos existe e é igual a $H_{\Lambda}^{-1}$, que é o raio de Hubble $(c=1)$. Ou seja, no caso de um universo em expansão acelerada, o horizonte de eventos coincide com o raio de Hubble, chamado também de horizonte de Hubble. Assim, nesse modelo qualquer evento que ocorrer a distâncias maiores que o raio de Hubble nunca poderá ser visto e nem influenciar o futuro de um observador.

\subsection{Descrição do universo pelo MCP e o universo jovem}

Nos capítulos anteriores apresentamos previsões geométricas do MCP. Essas previsões dependem da composição material do universo. Assim, temos que saber qual é a composição do universo em cada instante e como esta evolução de seu conteúdo ocorreu. Logo, o MCP não contempla somente seus aspectos geométricos, mas também sua composição material e história térmica.

Pelas previões anteriores vimos que o universo está em expansão. Isso nos faz pensar que anteriormente sua energia estava contida em uma região muito pequena, quente e densa. Por isso essa teoria também é chamada de Teoria do Big Bang Quente.

Podemos escrever as densidades relativas de matéria, energia e energia escura em função do redshift, dado em (2.11), da forma:

$$
\begin{cases}\Omega_{m}=\Omega_{m}^{0}(1+z)^{3,} & \text { matéria, } \\ \Omega_{\text {rad }}=\Omega_{\text {rad }}^{0}(1+z)^{4}, & \text { radiação, } \\ \Omega_{\Lambda}=\Omega_{\Lambda}^{0}(1+z)^{3(1+\omega)}, & \text { energia escura }\end{cases}
$$

onde a equação de estado da energia escura é constante, com $-1 \leq \omega \leq-1 / 3$. Pelas diferentes dependências dessas densidades com o redshift, podemos ver que cada uma dessas componentes dominará em uma diferente época, sendo que em,

$$
\begin{aligned}
& z_{e q}=\frac{\rho_{\text {crit }} \Omega_{m}}{\rho_{\text {rad }}^{0}}-1 \simeq 2,26 \times 10^{4} \Omega_{m} h_{75}^{2} \\
& z_{\Lambda}=\left(\frac{\Omega_{\Lambda}}{\Omega_{m}}\right)^{-1 / 3 \omega}-1
\end{aligned}
$$


em que $h_{75}^{2} \equiv H_{0} / 75 \mathrm{~km} \mathrm{~s}^{-1} \mathrm{Mpc}^{-1}$, correspondem aos redshifts em que o conteúdo material e o de radiação coincidem e em que o conteúdo material e de energia escura coincidem, que deve ter ocorrido entre $0,33 \lesssim z_{\Lambda} \lesssim 1,33$, sendo $\Omega_{m} \simeq 0,3$ e $\Omega_{\Lambda} \simeq 0,7$.

Assim, podemos ver que:

- $z>z_{e q} \sim 10^{4}$ : Época em que a radiação domina;

- $z_{e q}>z>z_{\Lambda}$ : Época em que a matéria domina;

- $z<z_{\Lambda}$ : Época em que a energia escura domina.

A determinação do redshift $z_{\Lambda}$ é um problema em aberto, uma vez que a energia escura não pode dominar o universo muito cedo, pois um período definido de dominação de matéria é necessário para a formação das estrutura. A questão de por que $z_{\Lambda} \sim O(1)$ é conhecida como problema da coincidência cósmica.

$\mathrm{Na}$ época em que a radiação domina o universo, podemos relacionar a temperatura com a densidade de energia, dada pela expressão da física estatística [16, 31]:

$$
\rho_{\text {rad }}(T)=\sum_{i=1}^{n} \alpha_{i} g_{i}\left(\frac{\pi^{2}}{30}\right) T^{4},
$$

em que $n$ é o número de partículas relativísticas (radiação), $g_{i}$ é o número de graus de liberdade de spin cada partícula e $\alpha_{i}=1,7 / 8$ para bósons ou férmions, respectivamente. Lembrando que $\rho_{\text {rad }} \propto a^{-4}$, podemos escrever a relação da temperatura com o redshift dada por:

$$
T_{\text {rad }}(z)=T_{\text {rad }}^{0}(1+z)
$$

Com essa relação podemos agora descrever a história do universo de acordo com sua temperatura em cada época.

Para termos uma descrição completa do universo, entendendo sua composição e evolução dessas componentes, um modelo de partículas é necessário para entendermos como o universo evoluiu desde o Big Bang até os dias atuais. O MCP inclui o modelo padrão das partículas elementares. Com essas idéias, podemos descrever aqui os principais pontos da história térmica do nosso universo, esquematizada na Figura 2.2:

- Era de Planck: A era de Planck se estende desde o começo do universo até épocas da ordem de $10^{-43} \mathrm{~s}$, com $T \sim 10^{32} \mathrm{~K} \sim 10^{19} \mathrm{GeV}$. Esse período não é descrito pelo MCP e não há praticamente nenhum entendimento atual do que acontece nesse período, em que a teoria da relatividade geral não tem mais validade e os efeitos quânticos dominam. 
- Inflação: Desde o fim da era de Planck, aproximadamente, até tempos da ordem de $10^{-32} s$, temos uma época de expansão acelerada do universo conhecida como inflação, que também não é descrita pelo MCP, mas é uma teoria criada para resolver os problemas que o MCP apresenta. Vamos ver esse período em mais detalhe no Capítulo 3.

- Nucleossiontese: Como o universo foi se esfriando, quando sua temperatura era da ordem $10^{8}-10^{9} \mathrm{~K}$, processos nucleares passam a ocorrer e a formar os elementos leves de nosso universo. Esse processo é conhecido como nucleossíntese primordial e ocorre entre $1 \mathrm{~s}$ a $500 \mathrm{~s}$ após o Big Bang. Todos os elementos químicos leves de nosso universo (que depois no núcleo das estrelas dão origem aos elementos pesados) são formados nessa época a partir dos prótons e dos neutrons, formando o hidrogênio $(H)$, deutério $\left(H^{2}\right.$ ou $\left.D\right), H^{3}$ e hélio $\left(H e^{4}\right)$, sendo que o hélio também é produzido por outros mecanismos após a nucleossíntese. Uma pequena fração de Lítio também é formada nesse período. As abundâncias desses elementos são um importante teste para o MCP, como citaremos a seguir.

- Recombinação e desacoplamento dos fótons: Depois da formação dos elementos químicos leves, o universo era dominado pela radiação (desde $z \gg 10^{4}$ ). Logo, matéria bariônica e radiação viviam em equilíbrio térmico nesse período, em que os fótons se espalhavam continuamente nos elétrons livres, mas sem ganhos ou perdas consideráveis de energia. Após isso, quando os elétrons livres se ligaram aos átomos de hidrogênio e de hélio, temos o fim desses espalhamentos. Esse período é chamado de recombinação e ocorre de $z=1400$, quando a temperatura é da ordem de $3800 K$, até $z=1100$, quando há o desacoplamento dos fótons da matéria. Isso ocorre quando a temperatura é da ordem de $3000 \mathrm{~K}$ e esse instante ("último" instante de interação dos fótons com a matéria) é chamado de superfície de último espalhamento. Esses fótons que se desacoplaram da matéria dão origem à RCF e essa superfície de último espalhamento é a origem da radiação que medimos hoje em dia.

- Era da matéria: Desde o desacoplamento dos fótons, a componente que dominou o universo foi a matéria (de $z=2 \sim 10^{3}$ ). Nesse período é que ocorre a formação das estruturas no universo via colapso gravitacional. Assim, ocorrem a formação das galáxias, aglomerados, etc.. 


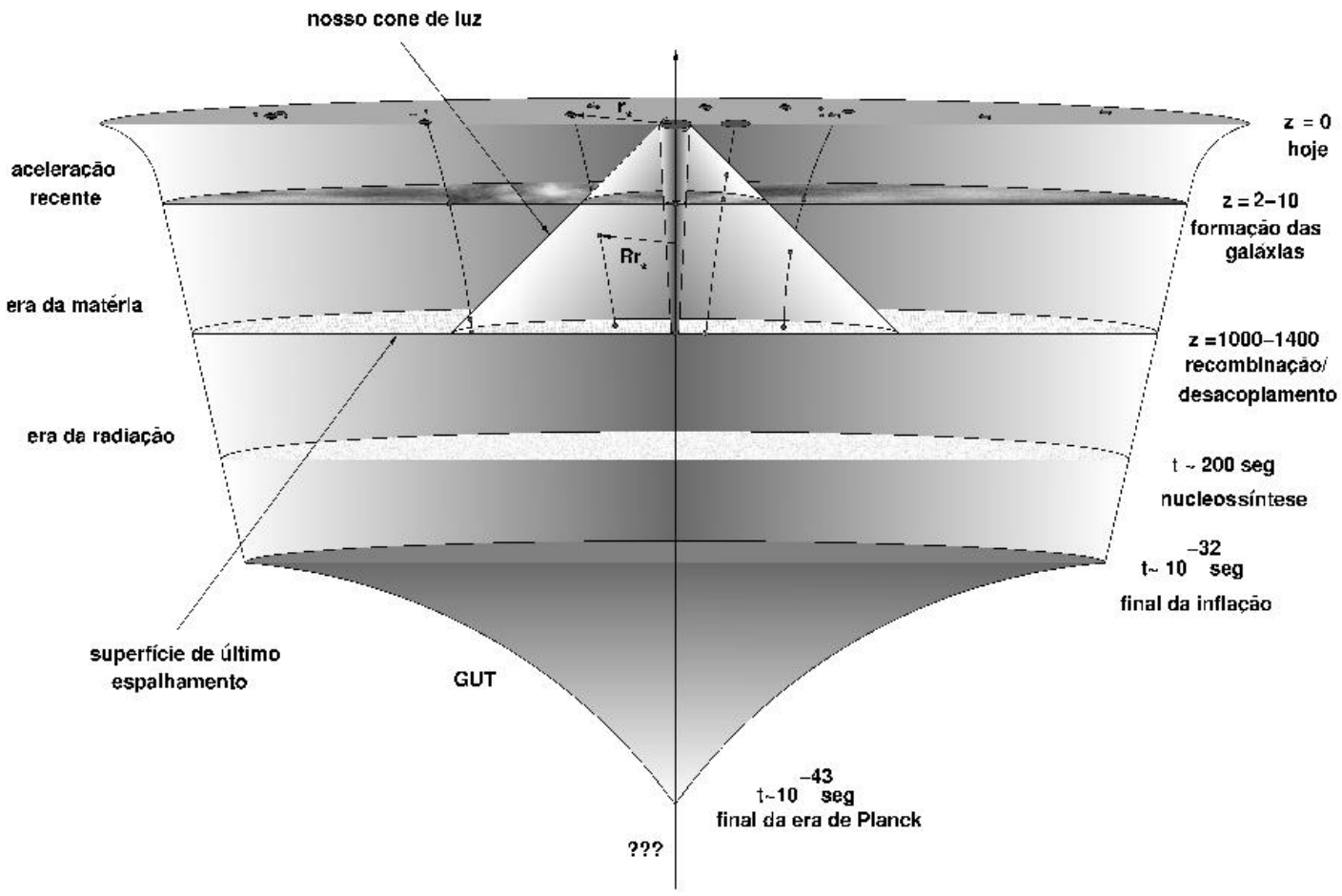

Figura 2.1: Figura retirada de [17]. Quadro esquemático da história térmica do universo.

\subsection{Testes do MCP}

O MCP foi construído através de previsões teóricas e observações experimentais do universo. Essas observações não só fazem parte da construção do modelo como são um importante suporte que comprova as previsões e o modelo em si. O MCP basea-se em três pilares obsevacionais: a expansão do universo, a abundância dos elementos leves e a RCF. Esses modelos apresentam também desvios importantes da teoria do Big Bang que reforçam a necessidade de teorias complementares a esse modelo.

\subsubsection{A Lei de Hubble}

Como já vimos na Seção 2.1.2, Edwin Hubble, em 1929, descobriu que o universo estava em expansão, medindo a velocidade de recessão de cefeidas ${ }^{3}$. As estrelas que Hubble conseguia enxergar naquela época eram bem próximas, distantes de nós não mais que

\footnotetext{
${ }^{3}$ Cefeidas são estrelas gigantes ou supergigantes amarelas, de 4 a 15 vezes mais massivas que o Sol e de 100 a 30000 vezes mais brilhantes, que têm luminosidade variável segundo períodos bem definidos. Também são chamadas de estrelas variáveis.
} 
alguns megaparsecs. Contudo, observações mais recentes, principalmente das supernovas do tipo Ia, complementaram o diagrama de Hubble para distâncias maiores e ajudaram a uma determinação mais precisa do parâmetro de Hubble.

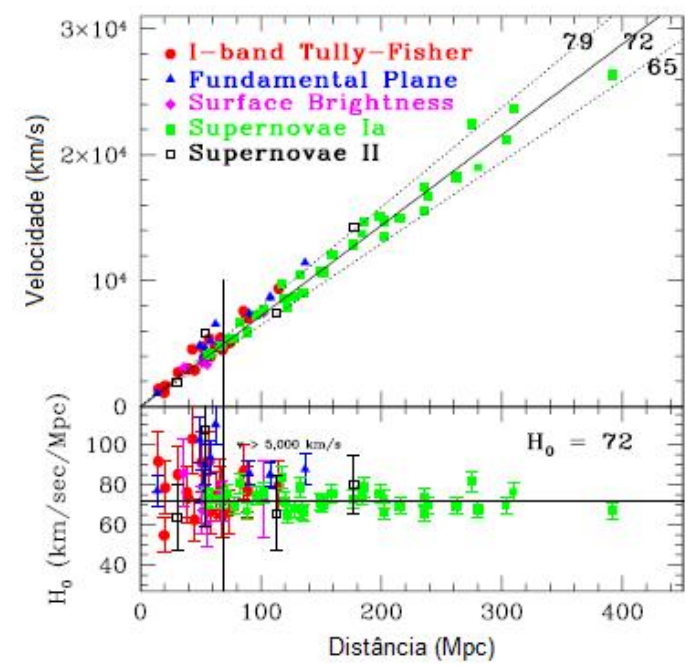

Figura 2.2: O gráfico mostra a medidas de cinco experimentos que comprovam a Lei de Hubble [18]. No painel superior, temos o gráfico da distância pela velocidade e no painel inferior, mostra que o parâmetro de Hubble se aproxima de $H_{0} \simeq 72 \mathrm{~km} \mathrm{~s}^{-1} \mathrm{Mpc}^{-1}$.

\subsubsection{Abundância dos Elementos Leves}

A abundância dos elementos leves, como hidrogênio, hélio e lítio, foi uma das primeiras previsões do MCP. A observação da abundância desse elementos é uma das principais testes que confirma o MCP. Como vimos, o universo era muito quente e denso em seu estágio inicial e, logo nos primeiros segundos, já estava cheio de prótons, neutrons, elétrons, pósitrons, fótons e neutrinos. Conforme foi se expandindo e se esfriando houve a formação do deutério (isótopo do hidrogênio) e durante os três primeiros minutos, a nucleossíntese primordial, em que esses núcleos de deutério se combinaram para formar He ( e também $\mathrm{He}^{3}$ ) e traços de lítio, criando um abundância relativa de He no plasma primordial de aproximadamente $25 \%$, enquanto que a do hidrogênio era de aproximadamente $75 \%$.

A abundância desses elementos leves depende da densidade da matéria presente nesse estágio inicial, como podemos ver na Figura 2.3.2. A observação da abundância de hidrogênio e hélio em galáxias e nebulosas está de acordo com a prevista pelo MCP. Essa é uma importante validação da teoria do Big Bang. 


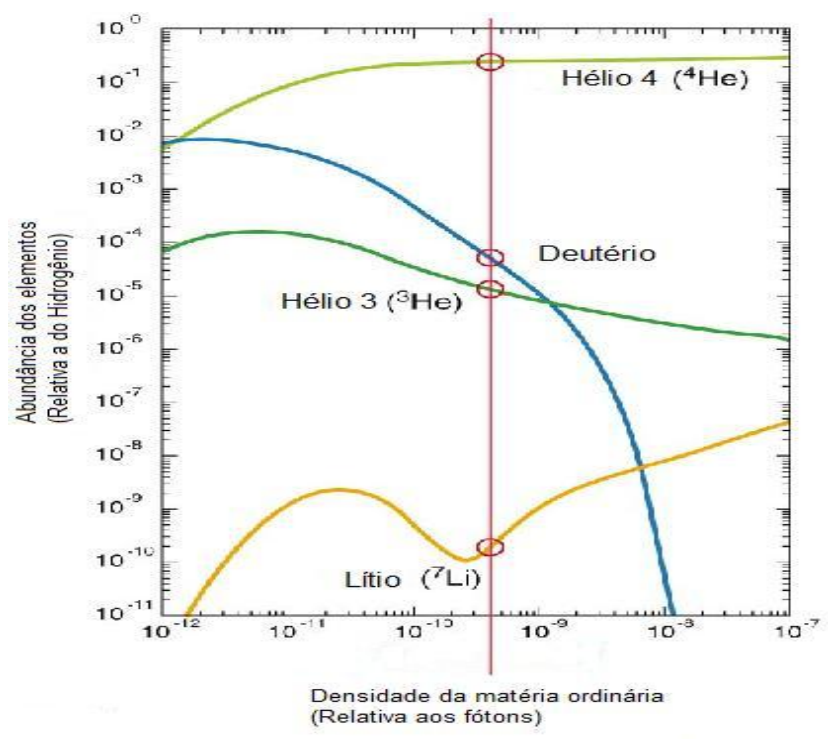

Figura 2.3: Gráfico da abundância de elementos leves em função da densidade da matéria ordinária no universo [21].

\subsubsection{A Radiação Cósmica de Fundo}

Como visto, a RCF foi prevista por George Gamow em 1948 e por Ralph Alpher e Robert Herman em 1950 e medida, acidentalmente, por Arno Penzias e Robert Wilson em 1965, o que lhes rendeu o prêmio Nobel em 1978. Hoje em dia, a detecção da RCF está longe de ser acidental e representa a principal fonte de dados observacionais para a cosmologia, uma vez que representa uma "foto" do universo quando tinha apenas 400.000 anos (aproximadamente) após o Big Bang.

Através dos satélites COBE, lançado em 1989, e WMAP, lançado em 2001, foi possível estudar a RCF e confirmar com uma incrível precisão as principais previsões dessa radiação. Foi medida, pelo $W M A P$, a temperatura da RCF com grande precisão, sendo ela $T=2,725 \pm 0,002 K[21,24]$. O experimento FIRAS no satélite COBE, mediu com uma precisão muito grande o espectro de frequências da $\mathrm{RCF}$, em forma de radiação de corpo negro. Este experimento mediu 34 pontos igualmente espaçados do espectro da RCF e a precisão era tão boa que as barras de erro obtidas eram tão pequenas que não podiam nem ser vistas no gráfico do espectro [9]. Como não existe nenhuma outra teoria alternativa que preveja esse espectro de energia, essa medida muito precisa do espectro de corpo negro da RCF representa mais uma importante validação do MCP.

Contudo, devido à grande precisão desses experimentos, foi possível verificar pequenas flutuações na temperatura dessa radiação em diferentes regiões do espaço. Essas flutua- 
ções podem nos fornecer grandes informações sobre parâmetros do MCP e sobre a origem das estruturas em larga escala que não é prevista pelo MCP.

\subsection{Problemas do MCP}

O MCP tenta descrever o universo e sua evolução. O sucesso desse modelo é inegável e bem testado, contudo, conforme a qualidade das observações vai aumentando, ou ainda o entendimento da teoria vai se aprofundando, percebemos que o MCP, apesar de uma boa aproximação, não é completo e apresenta problemas. Esse problemas envolvem principalmente as condições iniciais do universo, que podem modificar fortemente seu futuro e sua evolução. Podemos nomear alguns dos principais problemas da teoria do Big Bang: o problema da planura, o problema do horizonte, o problema da origem das estruturas, o problema da constante cosmológica, o problema dos monopolos magnéticos primordiais, entre outros. O problema da constante cosmológica, apesar de um dos mais importantes problemas atuais da cosmologia, ainda não apresenta solução, uma vez que as observações indicam um valor muito baixo (ou nulo), enquanto que a teoria quântica de campos prevê um valor muito alto, que difere $10^{44}$ vezes, no mínimo, desse valor observacional [19, 20]. Assim, mostraremos os outros problemas da teoria e no capítulo seguinte apresentaremos a teoria da inflação, que apresenta uma boa solução para essas questões.

\subsubsection{Problema da planura}

Como vimos na Seção 2.1.3, os dados observacionais atuais prevêem que o universo é aproximadamente plano, ou seja, sua densidade de energia é igual à densidade crítica, que é a densidade necessária para o universo se expandir. Em um universo dominado por matéria ou radiação e com uma curvatura espacial não nula, o valor de $\Omega \cong 1$ é um ponto instável na evolução do universo. Consideremos quais condições iniciais do universo seriam necessárias para que o universo apresentasse essa solução tão particular. Podemos ver esse problema de melhor maneira considerando a equação de Friedmann (2.20) e escrevendo-a em função da densidade total na forma:

$$
(\Omega-1) a^{2} H^{2}=k
$$

Sabendo que o parâmetro de curvatura é constante para todo instante, temos que $(\Omega-1) H^{2} a^{2}$ também é igual para qualquer tempo. Assim, se pensarmos que na era em que a matéria dominava, $(\Omega-1) \propto a$; e na época que a radiação dominava, $(\Omega-1) \propto a^{2}$. Como sabemos que hoje em dia $\left(\Omega_{0}-1\right)$ é da ordem da unidade e comparando com essa quantidade na era de Planck, fazendo a extrapolação que as equações de Einstein são válidas nesse período, temos a razão: 


$$
\frac{|\Omega-1|_{t=t_{p l}}}{\left|\Omega_{0}-1\right|} \approx \frac{a_{p l}^{2}}{a_{0}^{2}} \approx \frac{T_{0}^{2}}{T_{p l}^{2}}
$$

Sabendo que $T_{p l} \sim 10^{19} \mathrm{GeV}$ é a temperatura do universo no tempo de Planck e $T_{0} \sim$ $10^{-13} \mathrm{GeV}$ é a temperatura do universo atual, temos que essa razão é da ordem de $10^{-64}$. Se fizermos o mesmo cálculo para a época da nucleossíntese, em que a temperatura já é bem mais baixa, da ordem de $1 \mathrm{MeV}$, a razão das densidade é da ordem de $10^{-16}$. Com isso, podemos ver que para termos $\left(\Omega_{0}-1\right) \sim \mathcal{O}(1)$, o valor de $(\Omega-1)$ para o universo muito jovem tinha que ser muito pequeno, ajustado a valores muito próximos de zero. Esse problema também é chamado de problema do ajuste fino da curvatura espacial e pode ser resolvido pela teoria da inflação.

O problema da planura também é conhecido como problema da entropia. Isso ocorre pois, como vimos, a expansão do universo é adiabática e podemos escrever $\Omega-1=$ $k m_{p l} / S^{2 / 3} T^{2}$. Assim, podemos ver que, como a entropia do universo pelo presente volume de Hubble, durante um período dominado por radiação, é dada por [60]:

$$
S=\frac{4 \pi}{3} \frac{s}{H_{0}^{3}} \simeq 10^{90}
$$

temos que:

$$
|\Omega-1|_{t=t_{p l}}=\frac{m_{p l}^{2}}{T_{p l}^{2}} \frac{1}{S^{2 / 3}}=\frac{1}{S^{2 / 3}} \approx 10^{-60},
$$

ou seja, $(\Omega-1)$ é muito próximo de zero, uma vez que a entropia do universo é muito grande.

\subsubsection{Problema do horizonte}

Como vimos, a RCF apresenta quase a mesma temperatura em todos os pontos do universo observável, exceto por pequenos desvios. Contudo, existindo um horizonte de partículas hoje, regiões causalmente desconectadas no passado não teriam por que apresentar as mesmas características. Vamos ilustrar isso comparando o horizonte de partículas na época do desacoplamento do fótons com distância que a luz viajou desde a superfície de útimo espalhamento até nós. Como $a(t) \propto t^{2 / 3}$ nessa fase em que o universo é, em boa aproximação, dominado por matéria, essa razão é dada por [30]:

$$
\frac{\chi\left(t_{d e c}\right)}{\chi\left(t_{i}=t_{d e c}, t_{0}\right)}=\frac{H^{-1}}{\chi\left(t_{i}=t_{d e c}, t_{0}\right)} \simeq\left(\frac{t_{d e c}}{t_{0}}\right)^{1 / 3} \simeq 10^{-2} .
$$

Podemos projetar qualquer comprimento comóvel, $s$, na superfície de último espalhamento e obter o ângulo da esfera celeste ao qual ele corresponde. Essa relação é dada por: 


$$
\theta=\frac{s}{\left(\eta_{0}-\eta_{\text {dec }}\right)}
$$

lembrando que o tempo conforme é dado por $\eta(t)=\int^{t} d t / a$. Logo, no mapa da RCF, projetado na esfera celeste, que representa nosso horizonte de partículas de $360^{\circ}$, a razão (2.46) corresponde a um ângulo de aproximadamente um grau. Ou seja, na época de desacoplamento, o ângulo entre regiões com conexão causal era da ordem de um grau. Podemos calcular, assim, o número de regiões contidas dentro desse volume formado pela superfície de último espalhamento que têm conexão causal:

$$
N=\frac{4 \pi D^{3} / 3}{4 \pi(D \theta / 2)^{3}}=\frac{8}{\theta^{3}} \sim 10^{6} .
$$

Assim, como todas essas $10^{6}$ regiões da esfera celeste poderiam ter a mesma temperatura sem nem mesmo ter havido contato causal entre elas? Isso somente é possível se postularmos que as condições iniciais de todo o universo observável eram idênticas em todas essas $10^{6}$ regiões. Assim, precisamos de uma teoria em que essas regiões estejam causalmente conectadas no passado. Isso pode ser conseguido com a teoria da inflação, que veremos no próximo capítulo.

O problema do horizonte também é conhecido como problema da homogeneidade e isotropia [31]. Como o universo é homogêneo e isotrópico hoje em dia e, pelo MCP, inomogeneidades não podem ser dissolvidas pela expansão, então o universo atual deveria ter sido gerado a partir de uma região homogênea e isotrópica. Como o universo homogêneo e isotrópico é no mínimo do tamanho de seu horizonte, aproximadamente $c t_{0} \sim 10^{28} \mathrm{~cm}$, então a região homogênea e isotrópica da qual o universo se originou, em $t=t_{i}$, deveria ser da ordem de $\frac{a_{i}}{a_{0}} 10^{28} \mathrm{~cm}$. Comparando esse tamanho com o tamanho da região conectada causalmente (horizonte de eventos) no tempo inicial $c t_{i}$, temos que:

$$
\frac{t_{0}}{t_{i}} \frac{a_{i}}{a_{0}} \sim \frac{t_{0}}{t_{i}} \frac{T_{0}}{T_{i}} \sim 10^{28}
$$

em que o tempo inicial é tido como o fim da era de Plack, $t=t_{p l}=10^{-43} s, t_{0}$ é a idade de nosso universo estimada na Seção 2.1 .2 e assumimos que radiação domina desde $t_{p l}$. Assim, podemos ver que o tamanho do universo homogêneo e isotrópico inicial necessário excede a escala de causalidade por 28 ordens de magnitude. A única saída para o MCP seria postular como condição inicial que o universo fosse formado por regiões desconexas causalmente que fossem homogêneas e isotrópicas. Por ser também um problema da escala de causalidade, este problema e o problema do horizonte são equivalentes e são resolvidos pela teoria da inflação, que conecta causalmente essas regiões no início da inflação. 


\subsubsection{Problema da origem das estruturas}

O MCP prevê que o universo é, e sempre foi, homogêneo e isotrópico em largas escalas. Contudo, se isso foi sempre verdade, como teriam surgido as galáxias, aglomerados, planetas e outras estruturas que temos hoje em dia? Com o aumento da precisão na cosmologia observacional, principalmente com as medições da RCF, foi possível observar pequenas flutuações de temperatura nessa radiação, que poderiam dar origem às estruturas do universo. Essa formação pode ser descrita pela teoria clássica das perturbações cosmológicas, na qual em universos em expansão, formam-se as estruturas em larga escala por colapso gravitacional a partir de perturbações iniciais na densidade do universo. Contudo, nessa teoria as perturbações aparecem como condições iniciais impostas ao problema. Podemos medir da RCF, inclusive, que o espectro dessas perturbações é invariante de escala. Assim, necessitamos de uma teoria para essas perturbações primordiais, que também descreve por que seu espectro tem essa forma. O MCP postula essas "sementes" primordiais , assim como a forma de seu espectro, ajustando inclusive as condições iniciais para serem as mesmas para regiões do universo que, pela teoria, nunca seriam causalmente desconexas. Portanto, é necessária a apresentação de uma teoria que preveja essas perturbações a partir de primeiros princípios. Veremos a seguir que a inflação é uma teoria que satisfaz esses requisitos.

\subsubsection{Problema dos monopolos magnéticos}

Com a expansão do universo, sua temperatura cai e algumas simetrias que existiam a temperaturas mais altas são quebradas. Nesse período, é previsto, pelas Teorias de Grande Unificação, a presença de defeitos topológicos, manifestados como monopolos magnéticos. A produção desses monopolos é muito eficiente, com taxas de criação muito altas. A massa desses monopolos produzidos também é muito grande, cerca de $10^{16}$ vezes a massa do próton. Esses fatores, combinados, fariam com que há muito tempo o universo já houvesse colapsado gravitacionalmente devido a essa grande massa. A previsão de abundância desses monopolos supera em muito os limites observacionais, uma vez que nenhum monopolo foi observado ainda. Uma teoria satisfatória para o universo muito jovem deveria fazer com que esses tipos de defeitos fossem diluídos até densidades tão baixas que os tornariam inobserváveis. Novamente, a inflação dá conta dessa tarefa. 


\section{Capítulo 3}

\section{Inflação}

O MCP é a teoria que descreve nosso universo, prevendo sua evolução desde a nucleossíntese primordial até os dias atuais. Contudo, a teoria do Big Bang não explica os momentos iniciais em que o universo foi formado. Esse modelo apresenta alguns problemas ligados principalmente às suas condições iniciais ${ }^{1}$. Aparece, então, a Teoria da Inflação, em 1981, formulada pelo físico Alan Guth com o intuito de resolver os problemas das condições iniciais do Big Bang. Essa teoria evoluiu e, além de resolver esses problemas iniciais, fixou-se como uma boa teoria para o universo jovem, sendo a responsável pela criação das perturbações iniciais que, conseqüentemente, são as sementes para as estruturas em grande escala que temos hoje.

A primeira teoria da inflação formulada por Alan Guth chamava-se Inflação "Velha" (Old Inflation)[10]. Guth, um físico de partículas, criou essa teoria com a intenção de resolver o problema dos monopolos magnéticos da física de partículas. Estudando as propriedades das Teorias da Grande Unificação das forças ${ }^{2}$ (TGU), ele notou que essa teoria previa a criação de um grande número de monopolos magnéticos. Para resolver esse problema, Guth percebeu que uma quebra de simetria associada a um campo escalar no Modelo Padrão das Partículas Elementares deveria fazer com que o universo entrasse em um período de expansão rápida que seria responsável por "diluir" esses monopolos criados. A esse campo escalar foi dado o nome de inflaton. Pensando de outra maneira, o universo estava em um estado de falso vácuo com densidade de energia muito alta, em que o campo escalar estava no mínimo local de seu potencial, agindo como uma constante cosmológica. Em algum ponto, a inflação cessa devido ao tunelamento quântico desse campo para o mínimo global do potencial, que corresponde ao estado do nosso universo atual, o vácuo real. Esse decaimento ocorre em bolhas de vácuo real e a energia liberada desse falso vácuo reaquece o universo. Guth percebeu que esse modelo resolveria também

\footnotetext{
${ }^{1} \mathrm{O}$ MCP apresenta outros problemas também como o problema da constante cosmológica, o problema das componentes, entre outros.

${ }^{2}$ Teoria que unifica as três forças da natureza - eletromagnética, forte e fraca - a energias muito altas, correspondentes a temperaturas da ordem de $10^{28} \mathrm{~K}$.
} 
os outros problemas das condições iniciais do MCP. Contudo, esse modelo apresentava alguns problemas, uma vez que esse período inflacionário não poderia ocorrer no universo inteiro, mas em bolhas que não eram capazes reaquecer todo universo. Assim, o universo não poderia ser homogêneo e isotrópico como observamos hoje. Esse problema é chamado de problema do graceful exit.

Com esses problemas, o modelo foi logo substituído pela Inflação "Nova" ( New Inflation), formulada por Andre Linde [33] e independentemente por Andreas Albrecht e Paul Steinhardt [34], em que o universo se expande quase exponencialmente durante uma fase em que o inflaton rola lentamente em direção ao mínimo de seu potencial e, quando essa rolagem é mais lenta que a expansão do universo, temos a inflação. Esse modelo ainda apresenta problemas, uma vez que não é capaz de gerar um universo perfeitamente simétrico. Contudo, ele é a base para o modelo mais aceito e o que iremos estudar nessa dissertação, chamado de inflação caótica. A inflação nova, porém, já é um modelo capaz de gerar as flutuações quânticas no inflaton. Com um mecanismo inflacionário para a criação dessas flutuações, Viatcheslav Mukhanov e G. V. Chibisov [35] foram os primeiros a calcular essas flutuações quânticas, em um modelo de inflação criado por Starobinsky, e a provar que elas são a origem das estruturas em larga escala vistas atualmente. Essa é uma conseqüência da teoria da inflação de grande importância na física atual.

A teoria da inflação, apesar de ainda apresentar alguns aspectos a serem completados, estabelece-se como uma forte teoria para o universo primordial. Como a física como a conhecemos atualmente não é válida na era de Plack, vamos considerar o tempo inicial para a inflação como sendo o tempo de Planck, $t_{i}=t_{p l} \sim 10^{-43} s$. Vamos considerar também que, pela Relatividade Geral, as inomogeneidades primordiais não são dissolvidas pela expansão.

\subsection{Motivação}

Estabelecidos os problemas do MCP, como exposto no capítulo anterior, vamos apresentar algumas modificações com vista a contorná-los [31, 30]. Assim, em relação ao problema da planura (entropia), podemos pensar que, se o universo passasse por um período de expansão não-adiabática, poderia fornecer uma entropia (pelo presente volume de Hubble) tão grande tal qual observamos atualmente. Do problema do horizonte, podemos ver que se o universo passar por um período em que as escalas físicas (ou o horizonte de partículas) evoluírem mais rápido do que $H^{-1}$, ou seja, $\left[\chi\left(t_{i}=t_{d e c}, t_{0}\right)\right]^{\cdot}>\left(H^{-1}\right)^{\cdot}$, sendo que $\chi\left(t_{i}=t_{d e c}, t_{0}\right) \sim a$ e, pela definição da constante de Hubble, temos que a condição pode ser escrita como:

$$
\frac{d}{d t}\left(\frac{a}{H^{-1}}\right)=\ddot{a}>0
$$


Essa condição é muito forte, uma vez que nos permite ter uma interpretação geométrica dessa expansão acelerada: o comprimento comóvel de Hubble, $\mathcal{H}=H a$, está diminuindo durante esse período, enquanto que em qualquer outro período da evolução do universo (segundo o MCP) estaria aumentando. Essa característica é muito importante, uma vez que conforme o universo se expande seu comprimento de Hubble comóvel se torna menor em relação a essa expansão. Em outras palavras, a diminuição desse parâmetro nos permite dizer que, após esse período de expansão acelerada, o universo acessível para nós é menor do que era no início desse período. Assim, regiões distantes do universo eram conectadas causalmente antes desse período de expansão acelerada.

Dessa forma, podemos definir que é necessário um período de expansão acelerada antes do período de dominação da radiação para resolver os problemas do universo. Esse período é chamado de inflação. Traduzindo (3.1) em termo das equações de Friedmann (2.21), temos que, para que a inflação ocorra:

$$
\rho+3 p<0
$$

ou seja, $\omega<-1 / 3$. Essa é a condição necessária para que a gravidade seja "repulsiva" e não é satisfeita por nenhum tipo simples de matéria ou radiação.

Para estudarmos o período inflacionário, podemos considerar o caso em que $p=-\rho$ da constante cosmológica e, nesse caso, a solução das equações de Einstein é um universo de de Sitter. Logo, o fator de escala é dado por (2.34),

$$
a=a_{i} e^{H_{I}\left(t-t_{i}\right)}
$$

em que utilizamos o valor do parâmetro de Hubble da inflação, que podemos ver ser constante na inflação, $H(t)=\dot{a} / a=H_{I}$ e $t_{i}=t_{p l}$. Podemos reescrever o fator de escala da inflação em termos do tempo conforme sendo $a(\eta)=-1 / H_{I} \eta$. Porém, a solução exata de de Sitter e a inflação não podem ser consideradas a mesma coisa, uma vez que aquela solução não é capaz de produzir um graceful exit suave da inflação no período descrito pelo MCP.

Essa diferença entre o período de inflação e o universo de de Sitter pode ser caracterizada pelo tempo em que a expansão acelerada ocorre, o que determina a validade da teoria como descrição do universo primordial e resolve os problemas do MCP. Isso é usualmente descrito pelo número de e-folds, que representa quantos tempos de Hubble se passaram. Essa quantidade é definida da forma:

$$
N \equiv \int_{t_{i}}^{t_{f}} H d t=\ln \frac{a_{f}}{a_{i}},
$$

em que $a_{i}$ é o fator de escala no tempo inicial, $t_{i}=t_{p l}$ e $a_{f}$ é o fator de escala no tempo 
final da inflação. Vamos ver como essas hipóteses resolvem os problemas das condições iniciais do MCP.

\subsubsection{Problema da planura}

Como vimos na Seção 2.4.1, para obtermos um universo plano nos dias atuais (como mostram as observações) seria necessário que o universo, no início da era de dominação da radiação, tivesse um ajuste fino na curvatura da ordem de $|\Omega-1| \sim 10^{-60}$. A teoria da inflação resolve esse problema, uma vez que, durante a inflação, o fator de escala cresce exponencialmente e, como a densidade total é inversamente proporcional ao quadrado do fator de escala, como vemos de (2.42), então $\Omega-1$ deve ser muito pequeno.

Podemos ver isso da seguinte maneira: identificando o final da inflação como o começo da era de dominação da radiação, esse ajuste fino teria que valer também para o fim da inflação, denominado $t_{f}$. Com isso, uma vez que a curvatura é constante em todos os instantes e, como o parâmetro de Hubble é (quase)constante na inflação, de (2.42) temos a razão:

$$
|\Omega-1|_{t=t_{f}} \approx\left(\frac{a_{i}}{a_{f}}\right)^{2}|\Omega-1|_{t=t_{i}}=e^{-2 N}|\Omega-1|_{t=t_{i}},
$$

em que a última igualdade vem da definição do número de e-folds (3.4). Então, podemos ver que, mesmo que o valor de $|\Omega-1|$ no começo da inflação seja diferente de zero, com o crescimento exponencial do universo a densidade do universo se tornaria muito próxima da unidade no fim do período inflacionário. Dessa forma, a inflação resolve o problema da planura e consegue prever com grande precisão um universo plano com $\Omega_{0}=1$.

Podemos determinar a partir do problema da planura o número de e-folds necessário para o período inflacionário resolver esse problema. Contudo, esse limite é menos forte que o limite imposto pelo problema do horizonte, por exemplo.

Se pensarmos no problema da planura como um problema da entropia, podemos lembrar que o problema existe uma vez que a entropia em um volume comóvel se conserva, pois a expansão é adiabática. Assim, se em um intervalo de tempo finito no universo jovem essa expansão for não-adiabática, podemos postular que a entropia irá variar durante a inflação da forma:

$$
S_{f}=Z^{3} S_{i}
$$

em que $Z$ é um fator numérico. Após a inflação o universo volta a se expandir adiabaticamente, com $S=S_{f}$. Supondo que no início da inflação a entropia era da ordem da unidade, uma partícula por horizonte, temos que $Z=10^{30}$, uma vez que, como vimos anteriormente, $S=10^{90}$. Sabendo que, para radiação, a entropia por volume comóvel é 
dada por $S \sim(a T)^{3}[60]$, temos que, de (3.6):

$$
\begin{aligned}
& a_{f} T_{f} \approx 10^{30} a_{i} T_{i} \\
& \quad \Rightarrow \frac{a_{f}}{a_{i}}=e^{N} \approx 10^{30} \frac{T_{i}}{T_{f}} .
\end{aligned}
$$

Logo, $N$ é igual a, no mínimo, 70, uma vez que a razão das temperaturas inicial e final é maior do que um. Contudo, vale ressaltar que a expansão do universo durante a inflação é adiabática e por isso podemos usar durante esse período as equações de FRW. O período não adiabático refere-se à transição entre o fim da inflação e o início da era de dominação da radiação usual. Assim, a grande entropia do universo foi produzida durante essa transição de fase não-adiabática entre a inflação e a era de dominação da radiação posterior.

Podemos pensar, de maneira mais clara, que a inflação resolve o problema da planura uma vez que ela amplifica o raio de curvatura dado por:

$$
R=\frac{H^{-1}}{|\Omega-1|^{1 / 2}}=\left(\frac{a^{2}}{k}\right)^{1 / 2} .
$$

Assim, ela não muda a geometria global do universo que continuará sempre a mesma, mas sim sua geometria local, fazendo com que o universo pareça plano localmente, uma vez que seu raio de curvatura torna-se muito grande e indetectável após a inflação.

\subsubsection{Problema do horizonte}

Temos, com o problema do horizonte, que regiões do céu que têm as mesmas propriedades são, pelo MCP, regiões do universo causalmente desconexas no universo jovem. Assim, como vimos acima, a teoria da inflação faz com que essas regiões desconectadas causalmente fossem conectadas umas às outras no passado, uma vez que o comprimento de Hubble comóvel durante a inflação diminui. Essas regiões hoje distantes no céu e que apresentam as mesmas características estavam, então, muito perto umas das outras antes da inflação, dado que o domínio causal entre elas infla exponencialmente durante a inflação, mais rápido que a velocidade da luz. Agora, temos que saber o quanto esse domínio tem que inflar, ou seja, quanto tempo a inflação tem que durar para que conecte essas regiões e resolva o problema do horizonte.

Como, para resolvermos o problema do horizonte, queremos que o nosso universo observável estivesse dentro do raio de Hubble no início da inflação, temos que [30]:

$$
H_{0}^{-1} \lesssim \frac{a_{0}}{a_{i}} H_{I}^{-1}=\frac{a_{0}}{a_{f}} \frac{a_{f}}{a_{i}} H_{I}^{-1}=\frac{a_{0}}{a_{f}} e^{N} H_{I}^{-1}
$$

em que o índice 0 corresponde aos valores dos parâmetros atualmente. Sabendo que 
$a_{0}=1$,

$$
N \gtrsim \ln \left(\frac{a_{f} H_{I}}{a_{0} H_{0}}\right)=\ln \left(\frac{H_{I}}{T_{f} H_{0}}\right)
$$

em que, como vimos na época de domínio da radiação, $a=T^{-1}$ e assumindo que a inflação coincide com o fim da era da TGU, com $T_{T G U} \sim 10^{29} \mathrm{~K}, H_{I} \sim T_{T G U}^{2} / m_{p l} \sim 10^{56} \mathrm{~km} / \mathrm{s} / \mathrm{Mpc}$ e $H_{0} \sim 10 \mathrm{~km} / \mathrm{s} / M p c$, temos então que são necessários aproximadamente $N \gtrsim 60 e-f$ olds para resolver o problema do horizonte. Esse número é apenas uma estimativa e varia conforme o modelo de inflação que usamos, mas seu valor fica entre 60-70 e-folds.

Somente resolver o problema do horizonte anterior não é suficiente para garantir que a inflação nos fornecerá o universo homogêneo e isotrópico do MCP. Temos que garantir também que a inflação resolverá o problema da homogeneidade e isotropia. Como vimos que a inflação conecta causalmente regiões que, pelo MCP, eram consideradas causalmente desconectadas, vemos que o problema da homogeneidade e isotropia é resolvido, uma vez que o universo do MCP atual pode começar de uma pequena região (causal) homogênea e isotrópica que é aumentada violentamente durante esse período de expansão acelerada.

Podemos ver também que a condição inicial de que esse universo deveria ser homogêneo antes da inflação também pode ser contornada, uma vez que as inomogeneidades iniciais (ou seja, as partículas) quando passam por período de expansão acelerada, se têm comprimento de onda menor que o horizonte de eventos da inflação, $H^{-1}$, são esticadas exponencialmente até que fiquem maiores que o raio de Hubble do universo, quando param de crescer e são congeladas. As inomogeneidades com comprimento de onda maior que o raio de Hubble ficam congeladas até o universo se expandir mais e elas reentrarem no horizonte. Esse mecanismo da inflação faz com que, no fim do estágio inflacionário, todas as inomogeneidades deixem o horizonte sempre na mesma escala. Dessa forma, a inflação é responsável por homogeneizar (em grande escala) o universo. Falaremos com mais detalhe sobre esse assunto no Capítulo 4. Vale observar também que diversos estudos foram feitos sobre universos inicialmente anisotrópicos e a capacidade da inflação de isotropizá-los. Para mais informações nesse tópico, ver referências [37, 38].

\subsubsection{Problema dos monopolos magnéticos}

A teoria da inflação oferece uma explicação para o problema da ausência de monopolos magnéticos no universo. Vamos assumir que monopolos magnéticos fossem criados antes ou durante o período inflacionário. A expansão exponencial do universo durante a inflação diluiria a densidade desses monopolos criados até limites indetectáveis. Podemos ver isso supondo que a densidade de monopolos inicial nas TGU é $n\left(t_{T G U}\right) \sim 10^{82} \mathrm{~m}^{-3}$ [30]. Assim, após o fim da inflação, essa densidade seria: 


$$
n\left(t_{f}\right) \sim \mathrm{e}^{-3 N} 10^{82} \mathrm{~m}^{-3}=\mathrm{e}^{-300} 10^{82} \mathrm{~m}^{-3} \sim 5 \times 10^{-49} \mathrm{~m}^{-3} 15 \mathrm{pc}^{-3}
$$

em que supomos que a inflação tenha durado 100 e-folds e que $n / s$ é constante e portanto $n_{i} / n_{f} \propto\left(T_{i} / T_{f}\right)^{3} \propto\left(a_{i} / a_{f}\right)^{3} \sim e^{-3 N}$. Podemos ver então que esses monopolos magnéticos são extremamente diluídos durante a inflação. Se contarmos ainda com a expansão do universo ocorrida depois da inflação, podemos ver que esses monopolos diluir-se-ão ainda mais um pouco, tornando-se totalmente indetectáveis para nós.

\subsubsection{O problema da origem das estruturas}

O problema da origem das estruturas não foi o problema que motivou a construção da teoria da inflação. Contudo, certamente é a sua mais importante conseqüência. Devido à sua importância, estudaremos em detalhes a teoria das pertubações cosmológicas na inflação no Capítulo 4. Essa teoria nos fornece as sementes, flutuações primordiais, para as formação das estruturas em larga escala que observamos. Esse mecanismo também é responsável por restringir a escala de energia da inflação.

\subsection{O paradigma inflacionário: a inflação e o inflaton}

Como vimos, a inflação foi concebida como um período de expansão acelerada em que o universo está em um estado de falso vácuo e a energia desse vácuo age como uma constante cosmológica. Contudo, uma constante cosmológica não explica bem a inflação, uma vez que sabemos que a inflação teve um começo e um final. Isso faz com que essa energia do vácuo seja dinâmica. Assim, é necessário acharmos um candidato dinâmico que faça com que a gravidade seja efetivamente repulsiva ao invés de atrativa, ou seja, que tenha equação de estado, como vimos acima, $\omega<-1 / 3$. Como mencionado, nenhuma matéria usual é capaz de ter pressão negativa. Essa condição pode ser obtida se utilizarmos campos como o componente responsável pela inflação, uma vez que esses podem produzir densidades de energia capazes de imitar a constante cosmológica, mas apresentando uma evolução dinâmica. Vamos utilizar no caso um campo escalar, chamado de inflaton que nos fornece a equação de estado $\omega \cong-1$.

Campos escalares foram estudados em teoria quântica de campos muito antes de sua utilização em cosmologia e descrevem o campo de partículas com spin 0. Estes aparecem como uma escolha natural para modelar a inflação, uma vez que só dependem do tempo em universos homogêneos e porque são importantes para o mecanismo de quebra espontânea de simetria nas teorias de partículas, assim como nas TGUs. Foi por isso que Guth utilizou-os para resolver o problema dos monopolos magnéticos. Convém notar que não é necessária a utilização de campos escalares fundamentais e vamos supor que o campo "condensado escalar" é aproximadamente homogêneo. 
Para descrever a dinâmica da teoria da inflação estamos interessados na forma do TEM do inflaton, que pode ser calculado através do funcional de ação. Vamos definir um campo escalar real e homogêneo acoplado minimamente à gravidade com funcional de ação dado por [30, 49]:

$$
\begin{aligned}
S[\varphi] & =-\int d^{4} x \sqrt{-g} \mathcal{L} \\
& =-\int d^{4} x \sqrt{-g}\left[\frac{1}{2} \partial^{\mu} \varphi \partial_{\mu} \varphi+V(\varphi)\right],
\end{aligned}
$$

em que $\mathcal{L}$ é a densidade Lagrangeana, $-g$ é o determinante da métrica $g_{\mu \nu}$ e $V(\varphi)$ é o potencial. Para cada diferente teoria da inflação temos uma forma do potencial diferente. Temos alguns exemplos, como campo escalar massivo, $V(\varphi)=\frac{1}{2} m \varphi^{2}$; o potencial de Higgs, $V(\varphi)=\left(\varphi^{2}-M^{2}\right)^{2}$; o campo escalar de auto interação $V(\varphi)=\lambda \varphi^{4}$, entre outros. Vamos trabalhar por enquanto com a forma geral do potencial.

Variando essa ação em relação à métrica, obtemos o TEM da forma apresentada no Capítulo 2:

$$
T_{\nu}^{\mu}=\varphi^{, \mu} \varphi_{, \nu}-\left[\frac{1}{2} \varphi^{, \gamma} \varphi_{, \gamma}-V(\varphi)\right] \delta_{\nu}^{\mu} .
$$

Podemos reescrever esse tensor na forma de um TEM de um fluido ideal (2.15) se definir$\operatorname{mos} u^{\alpha} \equiv \varphi^{, \alpha} / \sqrt{\varphi^{, \gamma} \varphi_{, \gamma}}$ e escrevermos a densidade de energia e a pressão como:

$$
\begin{aligned}
\rho & \equiv \frac{1}{2} \varphi^{, \gamma} \varphi_{, \gamma}+V(\varphi)=\frac{1}{2} \dot{\varphi}^{2}+V(\varphi) \\
p & \equiv \frac{1}{2} \varphi^{, \gamma} \varphi_{, \gamma}-V(\varphi)=\frac{1}{2} \dot{\varphi}^{2}-V(\varphi),
\end{aligned}
$$

em que a derivada $\varphi^{, \gamma}$ se transforma em uma derivada temporal, pois nosso campo escalar é, por enquanto, homogêneo.

Para podermos descrever o período inflacionário, vamos estudar a dinâmica do inflaton no universo em expansão. Para isso derivamos a equação de movimento a partir da ação do campo escalar homogêneo, que nos fornece a conhecida equação de Klein Gordon:

$$
\ddot{\varphi}+3 H \dot{\varphi}+V^{\prime}(\varphi)=0,
$$

em que $V^{\prime}(\varphi)=d V / d \varphi$ e para a métrica de FRW, $\sqrt{-g}=a^{3}(t)$. Essa equação também pode ser derivada se substituirmos as equações (3.15) e (3.16) na equação da continuidade (2.22). Para determinarmos completamente a dinâmica do inflaton, obtemos mais uma equação se substituirmos a forma de densidade de energia e da pressão em função do campo escalar na equação de Friedmann (2.20): 


$$
H^{2}=\frac{8 \pi G}{3}\left[\frac{1}{2} \dot{\varphi}^{2}+V(\varphi)\right],
$$

em que não consideramos o termo da curvatura, uma vez que, como vimos no problema da planura, ele logo se tornará desprezível assim que a inflação começa. Podemos escrever essa equação também em função da massa de Planck, que no sistema de coordenadas que estamos utilizando é dada por $M_{p l}^{2}=1 / G$.

\subsubsection{Regime de rolagem lenta (Slow-Roll)}

Após descrevermos o período inflacionário em termos do inflaton, temos que determinar em que condições esse campo escalar será capaz de fornecer um período inflacionário para o universo. Para termos inflação, ou seja, expansão acelerada, temos que ter equação de estado $p<-\rho / 3$, ou, mais genericamente, $p \cong-\rho$, para inflação quase-exponencial. Pela definição em função do campo escalar de $\rho$ e $p$, podemos ver de (3.15) e (3.16) que para satisfazerem a equação de estado da inflação, é necessário que:

$$
\begin{aligned}
\ddot{a}>0 & \Leftrightarrow p \simeq-\rho \\
& \Leftrightarrow \dot{\varphi}^{2} \ll V(\varphi) .
\end{aligned}
$$

Ou seja, teremos inflação sempre que o potencial dominar. Essa condição quer dizer que o campo escalar está rolando lentamente pelo seu potencial. Por isso, esse regime é chamado de regime de rolagem lenta ou condição de rolagem lenta, que é a condição para que a inflação ocorra. Com essa condição podemos reduzir as equações que fornecem a dinâmica do inflaton na forma:

$$
\begin{aligned}
H^{2} & \simeq \frac{1}{3 m_{p l}^{2}} V(\varphi) \\
3 H \dot{\varphi} & \simeq-V^{\prime}(\varphi),
\end{aligned}
$$

em que $m_{p l}^{2}=1 / 8 \pi G$ é a massa de PLanck reduzida e considerando que a aceleração do campo é muito menor que sua velocidade:

$$
\ddot{\varphi} \ll 3 H \dot{\varphi}
$$

e por isso esse termo é desprezado. Essas equações restringem a forma do potencial, uma vez que a condição de rolagem lenta e a condição (3.22) podem ser reescritas, utilizando (3.20) e (3.21), da forma: 


$$
\begin{aligned}
\dot{\varphi}^{2} & \ll V(\varphi) \Rightarrow \frac{m_{p l}^{2}}{3}\left(\frac{V^{\prime}}{V}\right)^{2} \ll 1 \\
\ddot{\varphi} & \ll 3 H \dot{\varphi} \Rightarrow \frac{1}{3} \frac{V^{\prime \prime}}{H^{2}} \ll 1 .
\end{aligned}
$$

Com isso, definimos os parâmetros de rolagem lenta como sendo:

$$
\begin{aligned}
\epsilon & =\frac{m_{p l}^{2}}{2}\left(\frac{V^{\prime}}{V}\right)^{2}=-\frac{\dot{H}}{H^{2}}=\frac{1}{2 m_{p l}^{2}} \frac{\dot{\varphi}^{2}}{H^{2}} \\
\eta_{s r} & =\frac{1}{3} \frac{V^{\prime \prime}}{H^{2}}=m_{p l}^{2}\left(\frac{V^{\prime \prime}}{V}\right),
\end{aligned}
$$

em que utilizamos as equações dinâmicas da inflação para escrevê-los de diferentes formas, que nos permitem diferentes interpretações. Podemos ver que $\epsilon$ mede a inclinação do potencial e mostra o quanto a taxa de Hubble muda com o tempo durante a inflação. $\mathrm{O}$ segundo parâmetro, $\eta_{s r}$, nos fornece informação sobre a curvatura do potencial. Existe ainda um terceiro parâmetro definido como sendo $\delta=\eta_{s r}-\epsilon=-\ddot{\varphi} / H \dot{\varphi}$. Dessas definições é fácil ver que podemos reescrever a equação de Friedmann (2.21) da forma:

$$
\frac{\ddot{a}}{a}=H^{2}(1-\epsilon)
$$

e, para que tenhamos inflação, ou seja, $\ddot{a}>0$, temos que $\epsilon<1$.

É útil também na inflação reescrever esse parâmetros em termos do tempo conforme, sendo $d \eta=d t / a(t)$ e a derivada em relação ao tempo conforme denotada por " $"$ ":

$$
\begin{aligned}
& \epsilon=1-\frac{\mathcal{H}^{\prime}}{\mathcal{H}^{2}}=\frac{2}{m_{p l}^{2}}\left(\frac{\varphi^{\prime}}{\mathcal{H}}\right)^{2} \\
& \delta=1-\frac{\varphi^{\prime \prime}}{\mathcal{H} \varphi^{\prime}}
\end{aligned}
$$

Das condições de rolagem lenta modificadas (7.81) e (3.24), podemos ver que teremos inflação com rolagem lenta se:

$$
\begin{aligned}
\epsilon & \ll 1 \\
\left|\eta_{s r}\right| & \ll 1 \text { ou } \delta \ll 1 .
\end{aligned}
$$


Dessa condição temos que uma forma do período inflacionário acabar é se as condições de rolagem lenta forem violadas conforme o campo atinja um mínimo de seu potencial. Assim, a inflação ocorrerá até $\epsilon \sim 1$. Como a condição para que haja inflação é uma limitação menos forte que a condição para que ocorra inflação com rolagem lenta, é possível que um período inflacionário ocorra mesmo com as condições de rolagem lenta sendo violadas, contudo esse período seria muito curto.

Podemos observar nesse ponto o que já havíamos mencionado: que o parâmetro de Hubble é quase constante na inflação. Vamos escrevê-lo em termos dos parâmetros de rolagem lenta. Da definição de tempo conforme, podemos escrever:

$$
\eta \equiv \int \frac{d t}{a(t)}=\int \frac{d a}{a \mathcal{H}}=-\frac{1}{\mathcal{H}}+\int \epsilon \frac{d a}{a \mathcal{H}}
$$

Da condição para termos inflação com rolagem lenta (3.30) e, sabendo que $\epsilon$ é constante, temos que:

$$
\eta=-\frac{1}{\mathcal{H}}(1+\epsilon)+\mathcal{O}(2)
$$

em que podemos ver que o parâmetro de Hubble é quase constante durante o período inflacionário com rolagem lenta.

\subsubsection{Número de $e$-folds}

Baseado nesse modelo do campo escalar homogêneo responsável pela inflação, temos que definir o quanto a inflação deve durar desde seu início até seu final para que possa resolver os problemas das condições iniciais e fazer previsões corretas sobre a formação das estruturas em larga escala. Sendo $\varphi_{i}$ e $\varphi_{f}$ o valor do inflaton no início e no final da inflação, respectivamente, podemos calcular o número de e-folds que a inflação deve durar, considerando (3.4), da forma:

$$
\begin{aligned}
N & \equiv \int_{t_{i}}^{t_{f}} H d t \simeq H \int_{\varphi_{i}}^{\varphi_{f}} \frac{d \varphi}{\dot{\varphi}} \\
& \simeq-\frac{1}{m_{p l}^{2}} \int_{\varphi_{i}}^{\varphi_{f}} \frac{V}{V^{\prime}} d \varphi
\end{aligned}
$$

em que utilizamos as equações dinâmicas da inflação no regime de rolagem lenta. Podemos ver da equação acima que é possível calcular o número de e-folds sem que seja necessário resolver as equações de movimento e a equação de Friedmann da inflação. Como somente depende da forma do potencial, o número de e-folds varia muito para cada tipo de modelo 
inflacionário.

\subsubsection{Inflação caótica}

Desde o advento da teoria da inflação nos anos 80 uma enorme gama de modelos surgiu para tentar modelar esse período. Para uma descrição consistente da inflação esses modelos têm que explicar como eram as condições iniciais da inflação e como ocorre um graceful exit do período inflacionário para o período de expansão de Friedmann. Existem, assim, inúmeros candidatos que variam desde modelos como a inflação $k$, high derivative gravity e modelos de campos quânticos com potencial. Nesses modelos de campos quânticos com potencial, que é o caso que estamos estudando, podem ser implementadas modelagens mais complexas, com muitos campos escalares ou ainda com campos de diferentes naturezas, como campos vetoriais. Vamos estudar uma classe mais simples desses modelos, com um único campo escalar com potencial. Nesse caso, diferentes implementações da inflação serão dadas pelas diferentes formas que esse potencial pode assumir.

Convém ressaltar que, devido à dificuldades em se especificar o candidato mais exato para o inflaton, assim como devido a algumas incompatibilidades ainda não entendidas (como a energia do vácuo), a teoria da inflação desvincula-se da teoria de partículas no sentido que na cosmologia, ao invés de postularmos a natureza exata do inflaton com considerações da teoria de partículas, trabalhamos com a inflação como uma teoria que ocorre devido a uma energia do vácuo de um campo escalar qualquer. As características desse campo, assim como a distinção entre modelos de inflação são feitas observacionalmente.

O modelo de interesse que vamos estudar nessa dissertação é o modelo da inflação caótica, desenvolvido por Andrei Linde nos anos 80 [39]. Esse é um dos mais simples e amplos modelos de inflação, que aceita uma grande classe de potenciais. Suas principais características são: é um modelo de um único campo escalar; o potencial nesse modelo deve satisfazer, em alguma região, às condições de rolagem lenta e seu mínimo, que é onde a inflação deve acabar, deve ser igual zero; e tem condições iniciais muito gerais, que podem ser explicadas devido à grande flutuação durante a época de Planck. O nome inflação caótica, inclusive, refere-se a essa quase arbitrariedade das condições iniciais. Essa é uma grande qualidade desse modelo, pois "dribla" o problema de impor condições iniciais para a inflação, que teriam que ter sido construídas através de uma teoria para o período anterior ao inflacionário (o que já seria um desafio, uma vez que se trata da era de Planck) que por sua vez requereria a imposição de condições iniciais e o trabalho nunca acabaria. Podemos ver ainda que de (3.34), para qualquer potencial que satisfaça as condições de rolagem lenta (3.30) e (3.31), temos que $N \sim\left(\varphi_{i} / m_{p l}\right)^{2}$. Assim, como o valor do inflaton no final da inflação será menor que em seu início, temos que uma condição na inflação é que $\varphi_{i} \gg m_{p l}$. Mesmo com essa condição, $\varphi$ ainda é arbitrário.

Vários tipos de potenciais satisfazem as condições de rolagem lenta e podem ser im- 
plementados na inflação caótica. Apresentamos alguns deles aqui.

1. Inflação caótica polinomial: o potencial tem a forma $V \propto \varphi^{\alpha}$, contudo são mais utilizados os potenciais da forma:

$$
\begin{aligned}
V(\varphi) & =\frac{1}{2} m^{2} \varphi^{2} \\
V(\varphi) & =\lambda \varphi^{4},
\end{aligned}
$$

que correspondem aos já vistos modelo do campo escalar massivo, em que $m$ é a massa do inflaton, e do campo escalar de auto interação.

2. Inflação com lei de potência (power-law): nesse caso, o fator de escala evolui conforme uma lei de potência da forma $a(t) \propto t^{p}$, com $p>1$ e o potencial responsável por esse fator de escala é da forma:

$$
V(\varphi)=V_{0} \exp \left(\sqrt{\frac{16 \pi}{p}} \frac{\varphi}{m_{p l}}\right)
$$

3. Inflação intermediária: o potencial tem a forma, no regime de rolagem lenta, $V(\varphi) \propto \varphi^{-\beta}$, com $\beta=4\left(f^{-1}-1\right)$, o que nos fornece um fator de escala que evolui como $a(t) \propto \exp \left[\left(t / t_{0}\right)^{f}\right]$, com $0<f<1$.

4. Inflação "natural": o potencial tem a forma $V(\varphi)=V_{0}[1+\cos (\varphi / f)]$.

Convém notar que alguns desse potenciais podem modelar uma inflação infinita, uma vez que não apresentam um mínimo global, no qual a inflação deve terminar. Temos interesse, nessa dissertação, no modelo da inflação caótica polinomial, mais especificamente no modelo do campo escalar massivo.

\section{Inflação caótica polinomial: campo escalar massivo}

Vamos descrever o modelo de inflação caótica polinomial mais simples, em que o potencial é da forma:

$$
V(\varphi)=\frac{1}{2} m^{2} \varphi^{2}
$$

sendo $m$ a massa do inflaton.

Substituindo esse potencial na equação (3.18) em (3.17), temos que:

$$
\ddot{\varphi}+\sqrt{12 \pi}\left(\dot{\varphi}+m^{2} \varphi^{2}\right)^{1 / 2} \dot{\varphi}+m^{2} \varphi=0 .
$$

Essa equação, que representa a dinâmica do inflaton na inflação caótica com potencial (3.38), é uma equação não-linear de segunda ordem sem dependência explícita no tempo. 
Equações desse tipo podem ser muito complicadas de serem resolvidas. Contudo, podemos aqui fazer uma manipulação da equação, considerando que:

$$
\ddot{\varphi}=\dot{\varphi} \frac{d \dot{\varphi}}{d \varphi},
$$

que reduz a equação não-linear a uma equação diferencial de primeira ordem em $\dot{\varphi}(\varphi)$ :

$$
\frac{d \dot{\varphi}}{d \varphi}=-\frac{\sqrt{12 \pi}\left(\dot{\varphi}+m^{2} \varphi^{2}\right)^{1 / 2} \dot{\varphi}+m^{2} \varphi}{\dot{\varphi}} .
$$

Estudando o comportamento dessa solução via diagramas de fase, podemos ver que essa solução é um atrator, ou seja, possui trajetórias atratoras para as quais o sistema converge no tempo. Podemos ver na Figura 3.2.3, retirada de [31], que para diferentes pares $(\varphi, \dot{\varphi})$ de condições iniciais quaisquer, representadas pelas diferentes curvas, convergem para as curvas horizontais. Dessa forma, vemos que as soluções atratoras acontecem quando:

$$
\dot{\varphi}^{2} \ll m^{2} \varphi^{2},
$$

que é a condição de rolagem lenta imposta anteriormente. Com isso, vemos que a condição de rolagem lenta é naturalmente estabelecida para quaisquer condições iniciais. Por isso dizemos que a inflação caótica é um modelo que aceita condições iniciais muito gerais, uma vez que a solução é atratora e quaisquer condições iniciais convergem para as curvas em que o regime de rolagem lenta da inflação é válido. A inflação acaba quando $(\varphi, \dot{\varphi}) \approx 0$.

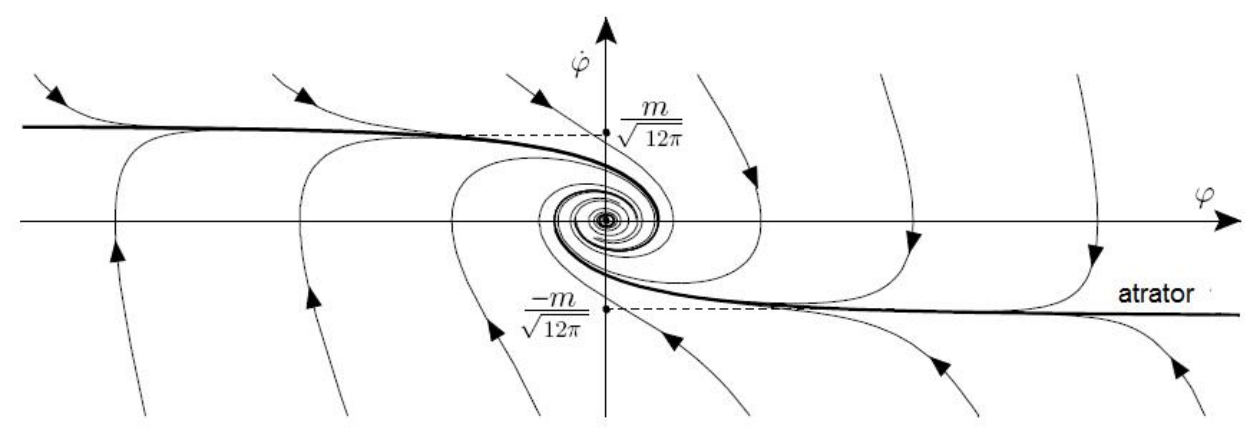

Figura 3.1: Figura retirada de [31]. Diagrama de fase da inflação caótica com potencial quadrático. As diferentes curvas representam diferentes pares de condições iniciais $(\varphi, \dot{\varphi})$. As curvas horizontais $\dot{\varphi}=0$ para onde as condições iniciais convergem representam o limite em que as condições de rolagem lenta são naturalmente definidas.

Estabelecida a generalidade desse modelo para as condições iniciais, vamos reescrever 
a teoria da inflação com rolagem lenta levando em consideração o potencial quadrático acima. Podemos reescrever as equações dinâmicas no regime de rolagem lenta (3.21) e (3.20):

$$
\begin{gathered}
3 H \dot{\varphi}+m^{2} \varphi=0 \\
H^{2}=\frac{1}{6} \frac{m^{2}}{m_{p l}^{2}} \varphi^{2} .
\end{gathered}
$$

Podemos também reescrever os parâmetros de rolagem lenta a partir desse potencial:

$$
\epsilon=\eta=\frac{2 m_{p l}^{2}}{\varphi^{2}}
$$

em que podemos ver que, para que ocorra inflação no regime de rolagem lenta, temos que $|\varphi| \gg \sqrt{2} m_{p l}\left(\gg m_{p l}\right)$. Dessa forma, vemos que para o potencial quadrático, também obtemos a restrição de que o campo tem que ser muito maior que a massa de Planck. Para não excedermos a escala de Planck, o que invalidaria nossa descrição do sistema, temos que $m \ll m_{p l}$.

As equações dinâmicas (3.43) e (3.44) são facilmente resolvidas, fornecendo:

$$
\begin{aligned}
& \varphi(t)=\varphi_{i}-m m_{p l} \sqrt{\frac{2}{3}} t \\
& a(t)=a_{i} \exp \left[\sqrt{\frac{1}{6}} \frac{m}{m_{p l}}\left(\varphi_{i} t-\sqrt{\frac{1}{6}} m m_{p l} t^{2}\right)\right] .
\end{aligned}
$$

Determinando o número de $e$-folds que a inflação dura nesse modelo, através de (3.4), temos que:

$$
N=\frac{1}{4} \frac{\varphi_{i}^{2}}{m_{p l}^{2}}-\frac{1}{2},
$$

e considerando um estimativa média da duração da inflação necessária para resolver os problemas das condições iniciais, temos que se $N \gtrsim 70 e-$ folds, então $\varphi_{i} \gtrsim 17 m_{p l}$.

Em resumo, podemos dizer que esse modelo descreve um universo que encontrava-se em um estado de vácuo que ainda não havia decaído para seu estado de mínimo global de energia, um falso vácuo. Essa energia do vácuo, como vimos acima, provoca uma aceleração da expansão do universo. Durante o período inflacionário, então, esse estado de vácuo vai decaindo até seu estado fundamental, conforme seu campo vai rolando lentamente até o mínimo do potencial (3.38). Nesse período as condições de rolagem lenta são obedecidas e, quando o campo atinge o mínimo do potencial, essas condições são violadas. Logo, o 
campo passa a oscilar em torno do mínimo do potencial e, para poder atingir o vácuo real do universo atual, acopla-se a um campo de matéria que decai em partículas que, por fim, reaquecem o universo. Essa fase final tem o nome de reaquecimento e será estudada em mais detalhes na próxima seção.

\subsection{Pré-aquecimento e Reaquecimento}

Como vimos, a teoria da inflação apresenta uma solução para os problemas do MCP e parece descrever bem nosso universo primordial. Contudo, logo após a inflação o universo apresenta-se quase sem partículas, ou seja, "frio" devido à sua grande expansão, com temperatura efetiva igual a zero. Para podermos obter o universo quente e denso requerido pelo MCP, é necessário que o universo seja reaquecido após o período inflacionário. Dessa forma, após a inflação o universo passa por um período chamado de reaquecimento. Esse ingrediente é necessário em qualquer teoria da inflação para nos garantir a passagem do período inflacionário ao período de dominação da radiação.

A teoria da inflação prevê que quase todas as partículas elementares existentes hoje (ou suas precursoras) foram criadas no reaquecimento. Contudo, esse mecanismo dinâmico de termalização do universo é pouco conhecido, uma vez que necessita de um conhecimento da Física de Partículas além do Modelo Padrão da Partículas Elementares. Muitas partes da teoria do reaquecimento ainda não são entendidas, mas existem modelos desse processo que nos fornecem uma boa idéia do que deve ter acontecido.

O período do reaquecimento consiste na oscilação do inflaton em torno de seu mínimo de potencial após ter rolado lentamente durante a inflação. As primeiras tentativas de se modelar o reaquecimento foram feitas em [40], no qual termos de fricção foram adicionados à equação de movimento do campo do inflaton para imitar a transferência de energia do inflaton para outro campo de matéria acoplado a ele. Avanços foram feitos aplicando-se essa teoria ao cenário da Inflação "Nova" em [41, 42], que utilizavam teoria perturbativa para poderem obter a temperatura do fim do reaquecimento $T_{\text {reaq }}$. Contudo, esse mecanismo tem suas limitações e não é capaz de descrever os estágios iniciais do reaquecimento.

Dessa forma, o estágio inicial da inflação, chamado de pré-aquecimento, foi entendido ocorrer em um regime de ressonância paramétrica larga (broad) em que a criação de partículas é extremamente eficiente [45]. Esse mecanismo é de importância central nessa dissertação e será introduzido nesse capítulo, enquanto a criação de partículas será estudada no Capítulo 6. A partir desse estágio, com a criação de partículas, a amplitude das oscilações vai se tornando menor e a ressonância torna-se cada vez mais estreita, em que a criação de partículas é ineficiente. Em suma, após a inflação o inflaton decai rapidamente em outras partículas por um mecanismo de ressonância paramétrica. Essas, conseqüentemente, decaem em outras espécies de partículas, que são as responsáveis por 
termalizar o universo. A teoria elementar do reaquecimento, que envolve a teoria perturbativa, descreve essa fase final do decaimento do inflaton e termalização do universo, finalizando o reaquecimento.

Primeiramente vamos descrever a evolução do campo do inflaton após a inflação [46]. Consideraremos em nossa análise o modelo da inflação caótica, com $V(\varphi)=\frac{1}{2} m \varphi^{2}$. Durante o período inflacionário, o inflaton obedece à equação de Klein Gordon (3.17) e às condições de rolagem lenta (3.23) e (3.24), uma vez que o campo do inflaton é $\varphi \gtrsim m_{p l}$. Conforme o inflaton vai descendo lentamente o potencial, seu valor fica menor que a massa de Planck e o termo de fricção $3 H \dot{\varphi}$ se torna cada vez menos importante. A inflação acaba quando $\varphi \sim m_{p l} / 2$. Assim, podemos descrever o reaquecimento como sendo o período que occorre após o regime de rolagem lenta, no qual o termo cinético se torna comparável ao potencial, $\dot{\varphi}^{2} / 2 \sim m^{2} \varphi^{2} / 2$, quando $\varphi=\varphi_{\text {reaq }} \simeq 1$. O termo de friç̧ão $3 H \dot{\varphi}$ torna-se pequeno e o inflaton desce o potencial aceleradamente de forma que as equações de movimento ficam da forma:

$$
\ddot{\varphi}+m^{2} \varphi \simeq 0
$$

em que a solução se aproxima assintoticamente de:

$$
\varphi(t) \simeq \Phi(t) \operatorname{sen}(m t)
$$

ou seja, o inflaton oscila em torno de seu mínimo do potencial.

Com essas condições, podemos ver que durante o reaquecimento o valor esperado da densidade de energia e da pressão, ficam:

$$
\langle\rho\rangle=\frac{1}{2} m^{2} \varphi^{2},\langle p\rangle=0
$$

que representam um universo dominado por matéria, como vimos no Capítulo 2. Ou seja, a oscilação do campo do inflaton imita um universo com equação de estado efetiva de matéria não-relativística com pressão nula. Dessa forma, sabemos de (2.34) que o fator de escala evolui como $a(t)=a_{0}^{m} t^{2 / 3}$ e a densidade de energia do inflaton decresce como $\rho_{\varphi} \propto a^{-3}$.

Podemos determinar a amplitude das oscilações do inflaton, sabendo que $H=\frac{2}{3} t$ :

$$
\Phi(t)=\frac{m_{p l}}{\sqrt{3 \pi} m t} \approx \frac{m_{p l}}{20 N}
$$

em que $N=m t / 2 \pi$ é o número de oscilações do inflaton desde o fim da inflação.

Nessas oscilações a energia armazenada no inflaton decai (é transferida) em partículas de outros campos que interagem entre si e entram em equilíbrio térmico à temperatura 
$T_{\text {reaq. }}$ O período de reaquecimento termina quando, através de um mecanismo de backreaction, a criação dessas partículas vai consumindo a energia do inflaton até ele atingir o mínimo de seu potencial, que no caso da inflação caótica é zero. Outro mecanismo que contribui para o fim desse período é o reespalhamento.

Primeiramente, iremos mostrar a teoria elementar do reaquecimento que envolve o decaimento perturbativo do inflaton. Contudo, nosso maior interesse nessa dissertação é em relação aos processos não-perturbativos do reaquecimento, em particular a época inicial do reaquecimento em que ocorre ressonância paramétrica e a produção de partículas é muito grande, chamado pré-aquecimento. Iremos, nesse capítulo, apenas introduzir os conceitos do pré-aquecimento, concentrando-nos mais nesse tópico no Capítulo 6.

\subsubsection{Teoria Elementar do Reaquecimento}

As primeiras teorias do reaquecimento [41, 42, 40, 43, 44] foram desenvolvidas baseadas no decaimento de um único corpo (single-body) em que o campo do inflaton era considerado como uma coleção de partículas escalares decaindo com probabilidades próprias finitas para cada uma delas. Para modelarmos esse decaimento, vamos introduzir a Lagrangeana do inflaton interagindo com um campo $\chi_{i}$, com mínimo do potencial em $\varphi=\sigma$. Fazendo o deslocamento $\varphi-\sigma \rightarrow \varphi$, temos que:

$$
\mathcal{L}=\frac{1}{2}\left(\partial_{\mu} \varphi\right)^{2}-V(\varphi)+\frac{1}{2} \sum_{i}[\left(\partial_{\mu} \chi_{i}\right)^{2}-\underbrace{\left(m_{\chi_{i}}^{2}(0)+g_{i}^{2} \varphi^{2}\right)}_{m_{\chi_{i}}^{2}} \chi_{i}^{2}]-\sum_{i} g_{i}^{2} \sigma \varphi \chi_{i}^{2}
$$

em que os acoplamentos $g_{i}$ e $\sigma$ têm dimensão de massa e $m_{\chi_{i}}^{2}$ representa a massa efetiva do campo $\chi_{i}$. Vamos utilizar aqui o potencial efetivo $V(\varphi)=\frac{1}{2} m^{2}(\varphi-\sigma)^{2}$ que, após o deslocamento, fica na forma conhecida $V(\varphi)=\frac{1}{2} m^{2} \varphi^{2}$. Essa Lagrangeana exibe acoplamentos que emergem naturalmente de teorias de calibre com quebra espontânea de simetria e, no caso em que $\sigma=0$, não temos quebra espontânea de simetria. Vamos considerar o campo do inflaton acoplado com um campo escalar $\chi$ e um campo fermiônico $\psi$, que descreve o campo dos férmions (partículas com spin semi-inteiro que obedecem a estatística de Fermi-Dirac). Podemos ver que os termos $g_{\chi} \sigma \varphi \chi^{2}$ e $g_{\psi} \sigma \varphi \psi \bar{\psi}$ são capazes de fornecer o decaimento do campo do inflaton massivo [45]. Para evitarmos instabilidade taquiônica, temos que ter:

$$
m^{2} \gg m_{\chi}^{2} \gg g_{\chi}^{2} \sigma \varphi
$$

sendo o mesmo válido para o campo fermiônico. Vamos assumir que após a inflação: 


$$
H \ll m,
$$

que é sempre válido no último estágio do reaquecimento, no qual vale a teoria elementar do reaquecimento que estamos desenvolvendo.

Vamos considerar agora a produção de partículas. Como mencionamos acima, para descrevermos fenomenologicamente o estágio final do reaquecimento, incluímos um termo de fricção na equação de movimento (3.17), que tem o papel de amortecer as oscilações do campo do inflaton, criando partículas. Obtemos assim a equação de um oscilador amortecido da forma:

$$
\ddot{\varphi}+3 H \dot{\varphi}+\Gamma_{\text {total }} \dot{\varphi}+m^{2} \varphi=0
$$

em que $\Gamma$ é a taxa de decaimento total do inflaton. Essa equação é válida somente para o caso em que o inflaton oscila rapidamente próximo ao seu mínimo.

As taxas de decaimento do campo do inflaton em pares de $\chi \chi$ e $\psi \bar{\psi}$, considerando o decaimento mais simples descrito pelo diagrama de Feynman de três pernas a nível de árvore em teoria de perturbação, é dado por [31, 46]:

$$
\begin{aligned}
\Gamma_{\varphi \rightarrow \chi \chi} & =\frac{g_{\chi}^{2} \sigma^{2}}{8 \pi m}, \\
\Gamma_{\varphi \rightarrow \psi \bar{\psi}} & =\frac{g_{\psi}^{2} m}{8 \pi} .
\end{aligned}
$$

Dessas relações, podemos notar que o decaimento do inflaton pode ser lento se as constantes de acoplamento forem pequenas, fazendo com que o processo do reaquecimento seja lento.

Sabendo que a solução da equação fenomenológica (3.56) também é da forma (3.50), podemos obter a forma da amplitude:

$$
\Phi(t)=\Phi_{0} \exp \left[-\frac{1}{2} \int d t(3 H+\Gamma)\right]=\frac{\Phi_{0}}{t} \mathrm{e}^{-\frac{1}{2} \Gamma_{\text {total }} t},
$$

em que na última igualdade utilizamos $H=\frac{2}{3} t$, sendo $\Gamma_{t o t a l}=\Gamma_{\varphi \rightarrow \chi \chi}+\Gamma_{\varphi \rightarrow \psi \bar{\psi}}$. Com isso, podemos estimar a densidade de número de partículas:

$$
n_{\varphi}=\frac{\rho}{m} \propto \mathrm{e}^{-\left(3 H+\Gamma_{t o t a l}\right) t} .
$$

Com essas soluções, podemos mostrar que a evolução da densidade de energia e densidade de número comóveis para o inflaton são: 


$$
\frac{d}{d t}\left(\rho a^{3}\right)=-\Gamma_{\text {total }} \rho a^{3}, \frac{d}{d t}\left(n_{\varphi} a^{3}\right)=-\Gamma_{t o t a l} n_{\varphi} a^{3}
$$

Após o decaimento do inflaton nessas partículas que são ultra-relativísticas, pois $m \gg$ $m_{\chi}$ e $m \gg m_{\psi}$, elas termalizam o universo. Com o decaimento total do inflaton, o equilíbrio térmico somente pode ser atingido se tivermos $\Gamma_{\text {total }}<H$. No começo do reaquecimento $\Gamma_{\text {total }} \ll H$ e o decaimento perturbativo pode ser desconsiderado. Após um certo número de oscilações do inflaton o decaimento passa a ser maior que o parâmetro de Hubble, $\Gamma_{\text {total }}>H$, uma vez que a densidade de energia das partículas criadas decresce com a expansão do universo muito mais rapidamente que a energia do inflaton e, dessa forma, o tempo de vida do inflaton é menor que a idade do universo, $\Gamma_{\text {total }}^{-1}<H^{-1}$. Nesse caso, o inflaton decai perturbativamente em partículas relativísticas $\chi_{i}$, com densidade de energia dada por (2.40). Assim, o reaquecimento acaba quando o decaimento se torna da ordem do parâmetro de Hubble, $\Gamma_{\text {total }} \sim H$. Assumindo que o equilíbrio térmico ocorre logo após decaimento do inflaton, podemos obter a temperatura no fim do reaquecimento de $(2.40)$ :

$$
T_{\text {reaq }} \simeq 0,2\left(\frac{100}{g^{*}}\right)^{1 / 4} \sqrt{\Gamma_{\text {total }} m_{p l}},
$$

em que $g^{*}$ é o número total de graus de liberdade que em modelos realísticos é da ordem de $10^{2}-10^{3}$.

Observações feitas pelo satélite WMAP das anisotropias da RCF estimam a massa do inflaton por $m \sim 10^{-6} m_{p l}$ [21], o que coloca um limite superior na temperatura final do reaquecimento $T_{\text {reaq }}<10^{6} \mathrm{GeV}$. Essa temperatura está abaixo da temperatura das TGU, o que garante que as simetrias das TGU não são reestabelecidas e o problema dos monopolos magnéticos não é alterado.

Essa descrição intuitiva e simples da teoria elementar do reaquecimento descreve bem o estágio final do reaquecimento para muitos modelos realísticos de inflação e, em particular, o caótico. Para mais detalhes dessa descrição ver [46].

\subsubsection{Pré-aquecimento}

O estágio final do reaquecimento, descrito acima, consiste em um processo perturbativo que nos fornece a temperatura final do reaquecimento devido à criação de partículas relativísticas que atingem equilíbrio térmico. Essa descrição é muito limitada e incapaz de descrever os estágios iniciais do reaquecimento em que as oscilações do inflaton são grandes e efeitos de condensação de Bose começam a se tornar importantes.

Vamos introduzir os estágios iniciais do reaquecimento em que a criação de partícu- 
las ocorre devido a um mecanismo não-perturbativo de ressonância parmétrica fora do equilíbrio térmico chamado pré-aquecimento. Investigaremos em detalhes esse mecanismo de ressonância paramétrica no Capítulo 6. Nesse estágio ocorre a criação de partículas escalares (bósons) devido à transferência de energia do campo do inflaton para campos escalares e outras partículas de maneira extremamente eficiente, sendo que consideramos que praticamente todas as partículas elementares do universo são criadas nesse estágio. Esse mecanismo é muito forte, uma vez que as partículas já criadas aumentam/estimulam a criação de novas partículas.

A ressonância paramétrica ocorre em dois diferentes regimes: de ressonância larga (broad) e estreita (narrow). O estágio de ressonância larga ocorre inicialmente para grandes amplitudes e é nele em que a criação de partículas é altamente eficiente. Conforme a amplitude das oscilações vai se tornando menor, as bandas de ressonância vão se tornando mais estreitas e temos o regime de ressonância estreita ou narrow. A criação de partículas no regime de ressonância ampla é muito grande e explosiva e férmions não são criados nesse períodos, uma vez que, pelo princípio de exclusão de Pauli, não podemos ter o crescimento explosivo de férmions.

Se incluirmos a expansão do universo na análise do pré-aquecimento, a criação de partículas ocorre em um regime de ressonância estocástica, uma vez que o termo de fricção que leva em conta a expansão do universo modifica a amplitude das oscilações do inflaton, o que faz com que o número de partículas cresça exponencialmente (em média), embora em alguns momentos possa decrescer, o que é característico de um processo estocástico. Essa ressonância estocástica somente ocorre no período inicial de ressonância paramétrica larga (broad) e conforme as oscilações tornam-se menores, efeitos da expansão do universo vão se tornando desprezíveis.

O mecanismo de ressonância paramétrica vai se tornando cada vez menos importante conforme efeitos de backreaction e reespalhamentos passam a ser não-desprezíveis e, em seguida, a teoria perturbativa torna-se uma boa descrição para o reaquecimento. 


\section{Capítulo 4}

\section{Teoria das Perturbações Cosmológicas}

No MCP consideramos o universo como homogêneo e isotrópico em grandes escalas, o que é garantido por medidas atuais de catálogos de galáxias [26, 27] e pelas medidas da RCF, que nos mostram que o universo era muito homogêneo e isotrópico na época da recombinação. Contudo, em nosso universo atual essa homogeneidade e isotropia é apenas uma aproximação [31], uma vez que observamos, em pequenas escalas, planetas, galáxias e outras estruturas não-lineares e, em escalas maiores que $100 \mathrm{Mpc}$, inomogeneidades como voids, grandes espaços vazios no universo, filamentos de galáxias, entre outros. O advento da cosmologia de precisão com o satélite COBE [9] e, com ainda mais precisão, o satélite WMAP [21], tornou possível detectar na RCF também desvios dessa homogeneidade, devido a pequenas flutuações na temperatura dessa radiação, que indicam perturbações da densidade de energia do universo na época da recombinação. Esses desvios na densidade de energia, porém, são muito pequenos, da ordem de uma parte em $10^{5}$ em relação ao fundo homogêneo. Dessa forma pensamos: como essas inomogeneidades tão pequenas surgiram em nosso universo e de que forma elas foram capazes de formar as estruturas em larga escala que observamos hoje em dia?

Vamos estudar então como essas inomogeneidades foram formadas e como se deu sua evolução até os dias atuais. Isso será feito através da caracterização dessas perturbações e de sua análise, linearizando essas perturbações, uma vez que tratamos de pequenas inomogeneidades em um fundo homogêneo e isotrópico. Dessa forma chamamos de Teoria das Perturbações Cosmológicas o estudo da teoria das perturbações gravitacionais linearizadas em um universo em expansão.

Primeiramente, iremos analisar a evolução de pequenas inomogeneidades presentes em um universo de FRW. Vamos estudar esse mecanismo na Teoria Clássica das Perturbações Cosmológicas. Determinado como essas inomogeneidades, inicialmente postuladas, se comportam, partiremos para a descrição de como elas se formam. A teoria da inflação é a responsável por esse mecanismo, que é considerado um tema central para a cosmologia moderna e representa uma das descobertas de maior importância da teoria inflacionária. Veremos esse tópico com mais detalhes quando descrevermos a Teoria Quântica das 


\subsection{Teoria Clássica das Perturbações Cosmológicas}

Antes mesmo do advento da teoria da inflação as perturbações cosmológicas já eram estudadas. As perturbações iniciais eram postuladas e o mecanismo de formação das estruturas em larga escala eram estudados baseados em suas propriedades gravitacionais. Devido ao caráter atrativo da gravidade a partir dessas pequenas inomogeneidades, que constituem zonas de maior concentração de matéria, atraem mais e mais matéria, por meio do colapso gravitacional, de forma que essa densidade cresce. Chamamos esse mecanismo de instabilidade gravitacional e é graças a ele que as estruturas são formadas.

O crescimento dessas perturbações iniciais foi descrito inicialmente utilizando a teoria newtoniana. Nessa teoria são descritas partículas não-relativísticas em escalas que não excedem o horizonte de Hubble, ou seja, perturbações que aconteceram em épocas tardias do universo. Essa descrição tem o intuito de nos fornecer uma percepção do comportamento das perturbações e de como analisá-las. Podemos ver essa teoria desenvolvida em [31]. Contudo, para uma descrição mais completa de sua evolução, sem ser necessário discernir entre o tipo de matéria e a escala da perturbação, vamos estudar as perturbações no contexto da relatividade geral [48, 49, 31].

\subsubsection{Teoria das Perturbações na relatividade geral}

O estudo das perturbações cosmológicas na aproximação da gravitação newtoniana é importante, pois nos dá uma boa noção da evolução das perturbações para a formação das estruturas em larga escala em tempos mais tardios do nosso universo, ou em escalas menores que o comprimento de Hubble. Para uma descrição mais completa das perturbações em nosso universo é necessário utilizarmos uma teoria que descreve quaisquer tipo de matéria em quaisquer escalas. A aproximação newtoniana falha na descrição de perturbações em escalas maiores que o raio de Hubble, uma vez que nessas escalas as perturbações da métrica devem ser consideradas. Como a relatividade geral acopla, por meio das equações de Einstein, a matéria e a geometria, perturbações da matéria (e da energia) implicam em perturbações da geometria, descrita pela métrica. Dessa forma, os efeitos da relatividade geral devem ser levados em conta.

A descrição das perturbações levando em conta a relatividade geral é uma tarefa um pouco mais complicada, uma vez que essa teoria tem simetria por transformações que levam um sistema de coordenadas em outro e dessa forma permite uma grande liberdade na escolha das coordenadas, o que deve ser tomado com muito cuidado para que não apareçam modos fictícios das perturbações, devido à mudança de coordenadas.

A relatividade geral é uma teoria de caráter não-linear, por construção. Logo, para 
podermos estudar a teoria linear das perturbações, devemos levar em conta perturbações muito pequenas em relação ao fundo. Outra dificuldade se apresenta uma vez que a métrica na relatividade geral, que descreve o espaço-tempo, é um tensor de ordem dois contendo diferentes modos perturbativos que devem ser analisados separadamente na teoria linear.

\subsubsection{Classificando os modos das perturbações}

Como na teoria clássica das perturbações, postulamos a existência de pequenas perturbações da matéria e energia que, pelas equações de Einstein (2.14), induz pequenas perturbações na métrica do espaço-tempo:

$$
g_{\mu \nu} \rightarrow g_{\mu \nu}^{(0)}+\delta g_{\mu \nu}
$$

em que $\left|\delta g_{\mu \nu}\right| \ll\left|g_{\mu \nu}^{(0)}\right|$. O fato dessas perturbações serem pequenas é que nos permite linearizar as equações de Einstein. Introduzindo essa perturbação no elemento de linha (2.2), podemos escrever, em tempo conforme:

$$
\begin{aligned}
d s^{2} & =\left[g_{\mu \nu}^{(0)}(\eta)+\delta g_{\mu \nu}(\eta, \mathbf{x})\right] d x^{\mu} d x^{\nu} \\
& =d s_{0}^{2}+d s^{\prime 2}
\end{aligned}
$$

em que:

$$
d s_{0}^{2}=g_{\mu \nu}^{(0)}(\eta) d x^{\mu} d x^{\nu}=a^{2}(\eta)\left(d \eta^{2}-\delta_{i j} d x^{i} d x^{j}\right),
$$

é a métrica de um universo de FRW plano e $d s^{\prime 2}=\delta g_{\mu \nu}(\eta, \mathbf{x}) d x^{\mu} d x^{\nu}$ representa o elemento de linha das pertubações da métrica. Sabemos que $d s_{0}^{2}$ é invariante por rotações e translações no espaço tridimensional, uma vez que nosso espaço de FRW de fundo é homogêneo e isotrópico. Vamos utilizar essa mesma simetria para classificarmos os tipos de perturbações da métrica. Sabemos que a métrica é simétrica, de forma que $\delta g_{\mu \nu}$ terá dez graus de liberdade livres.

Primeiramente, vamos analisar o modo $\delta g_{00}$ que se transforma como um escalar em relação a essas transformações e pode ser escrito em função do escalar $\phi$ da forma:

$$
\delta g_{00}(\eta, \mathbf{x})=2 a^{2}(\eta) \phi(\eta, \mathbf{x})
$$

O componente não diagonal da métrica $\delta g_{0 i}$ representa um modo vetorial e somente existem duas maneiras de se escrever um vetor, como o gradiente de um escalar e através de um vetor com divergência nula, da forma: 


$$
\delta g_{0 i}(\eta, \mathbf{x})=2 a^{2}(\eta)\left(B_{, i}+S_{i}\right),
$$

em que $B$ é um escalar e $S_{i}$ é um vetor tal que $S_{i}^{, i}=0$, pois se $S_{i}^{, i} \neq 0$, seria possível decompor $S_{i}$ em uma parte vetorial com divergente igual a zero e um escalar. Assim, $S_{i}^{, i}=0$ para que a decomposição acima seja única e irredutível.

A componente $\delta g_{i j}$ comporta-se como um tensor em relação às transformações de simetria. Dessa forma, existem cinco maneiras irredutíveis de se construir um tensor:

$$
\delta g_{i j}(\eta, \mathbf{x})=2 a^{2}(\eta)\left(2 \psi \delta_{i j}+2 E_{, i j}+F_{i, j}+F_{j, i}+h_{i j}\right)(\eta, \mathbf{x})
$$

em que $\psi(\eta, \mathbf{x})$ e $E(\eta, \mathbf{x})$ são escalares que podem determinar um tensor de maneiras distintas, multiplicado por um tensor unitário ou aplicando o laplaciano, respectivamente. Para construirmos um tensor a partir de um vetor, $F_{i}(\eta, \mathbf{x})$, é necessário que esse vetor tenha divergência nula, para sua descrição ser irredutível, como vimos acima. Por fim, $h_{i j}(\eta, \mathbf{x})$ é um tensor que, para ter uma descrição irredutível, ou seja, para não se decompor em novos vetores ou escalares, tem que ter traço nulo e ser transverso:

$$
h_{i}^{i}=0, h_{j, i}^{i}=0 \text {. }
$$

Com isso, podemos dividir as perturbações da métrica em seu modos escalares, vetoriais e tensoriais, que na teoria linear da perturbações não são acoplados entre si. Assim, podemos estudar a evolução de cada um deles independentemente. Temos os modos escalares, compostos por quatro funções escalares, escritos como:

$$
\delta g_{\mu \nu}^{\text {escalar }}=a^{2}\left(\begin{array}{cc}
2 \phi & -B_{, i} \\
-B_{, i} & 2\left(\psi \delta_{i j}-E_{i j}\right)
\end{array}\right) .
$$

Esses modos são os de maior importância para a formação de estruturas, uma vez que eles são acoplados às inomogeneidades da densidade de energia e são os únicos no qual o mecanismo de instabilidade gravitacional age. O elemento de linha das perturbações escalares é escrito como:

$$
d s^{2}=a^{2}(\eta)\left\{(1+2 \phi) d \eta^{2}+2 B_{, i} d \eta d x^{i}+\left[(1-2 \psi) \delta_{i j}-2 E_{, i j}\right] d x^{i} d x^{j}\right\}
$$

Temos também os modos vetoriais:

$$
\delta g_{\mu \nu}^{\text {vetorial }}=a^{2}\left(\begin{array}{cc}
0 & -S_{i} \\
-S_{i} & F_{i, j}+F_{j, i}
\end{array}\right),
$$

que exibem quatro graus de liberdade, descritos por dois vetores. Veremos aqui que nessa descrição as perturbações vetoriais não se acoplam às perturbações da matéria e, dessa 
forma, não têm papel na evolução das perturbações. O elemento de linha que descreve esse modo perturbativo é dado por:

$$
d s^{2}=a^{2}(\eta)\left[d \eta^{2}+2 S_{i} d \eta d x^{i}-\left(\delta_{i j}-F_{i, j}-F_{j, i}\right) d x^{i} d x^{j}\right] .
$$

Por fim, podemos representar os dois modos tensoriais:

$$
\delta g_{\mu \nu}^{\text {tensorial }}=a^{2}\left(\begin{array}{cc}
0 & 0 \\
0 & -h_{i j}
\end{array}\right),
$$

que representam dois estados polarizados das ondas gravitacionais. Na aproximação linear, veremos que as ondas gravitacionais não se acoplam às perturbações da matéria. Outra maneira de escrever a métrica desse modo é por meio de seu elemento de linha dado por:

$$
d s^{2}=a^{2}(\eta)\left[d \eta^{2}-\left(\delta_{i j}-h_{i j}\right) d x^{i} d x^{j}\right]
$$

\subsubsection{Transformações de calibre, sistema de coordenadas e variáveis inde- pendentes}

Uma vez que a relatividade geral permite uma liberdade na escolha do sistema de coordenadas, é importante sabermos como a métrica é alterada devido a essas mudanças para que possamos identificar os modos que não têm significado físico. Vamos fazer uma transformação de coordenadas infinitesimal:

$$
x^{\mu} \rightarrow \tilde{x}^{\mu}=x^{\mu}+\xi^{\mu},
$$

que apresenta quatro graus de liberdade, representados pela componente temporal $\xi^{0}$, que dá origem a perturbações escalares da métrica e pela componente espacial $\xi^{i}$, que pode ser decomposta de maneira única em:

$$
\xi^{i}=\xi_{\perp}^{i}+\varsigma^{i},
$$

em que $\xi_{\perp}^{i}$ é um vetor com divergência nula e $\varsigma$ é uma função escalar. Assim, a componente vetorial de divergência nula tem dois graus de liberdade que contribuem para as perturbações vetoriais e mais um modo escalar, $\varsigma$. Vemos que uma transformação de coordenadas não induz modos tensoriais, o que já nos faz ver que as perturbações tensoriais são invariantes por transformações de coordenadas.

Sabendo que a métrica se comporta, sob uma transformação de coordenadas, da forma: 


$$
\begin{aligned}
\tilde{g}_{\mu \nu}\left(\tilde{x}^{\rho}\right) & =\frac{\partial x^{\gamma}}{\partial \tilde{x}^{\mu}} \frac{\partial x^{\delta}}{\partial \tilde{x}^{\nu}} g_{\gamma \delta}\left(\tilde{x}^{\rho}\right) \\
& \approx g_{\mu \nu}^{(0)}\left(x^{\rho}\right)+\delta g_{\mu \nu}-g_{\mu \delta}^{(0)} \xi_{, \nu}^{\delta}-g_{\gamma \nu}^{(0)} \xi_{, \mu}^{\gamma} \\
& =g_{\mu \nu}^{(0)}\left(\tilde{x}^{\rho}\right)+\delta \tilde{g}_{\mu \nu}\left(\tilde{x}^{\rho}\right)
\end{aligned}
$$

na aproximação linear em $\delta g$ e $\xi$, podemos relacionar (4.18) e (4.19), obtendo:

$$
\delta \tilde{g}_{\mu \nu}=\delta g_{\mu \nu}-g_{\mu \delta}^{(0)} \xi_{, \nu}^{\delta}-g_{\gamma \nu}^{(0)} \xi_{, \mu}^{\gamma}-g_{\mu \nu, \gamma}^{(0)} \xi^{\gamma}
$$

uma vez que a métrica usual de Friedmann nas novas coordenadas é escrita na aproximação linear da forma:

$$
g_{\mu \nu}^{(0)}\left(x^{\rho}\right) \approx g_{\mu \nu}^{(0)}\left(\tilde{x}^{\rho}\right)-g_{\mu \nu, \gamma}^{(0)} \xi^{\gamma}
$$

Com isso, podemos escrever as componentes escalares, vetoriais e tensoriais da métrica transformada, conhecida a métrica de FRW plana de fundo, $g_{00}^{(0)}=a^{2}(\eta)$ e $g_{i j}^{(0)}=$ $-a^{2}(\eta) \delta_{i j}$

$$
\begin{aligned}
& \delta \tilde{g}_{00}=\delta g_{00}-2 a\left(a \xi^{0}\right)^{\prime}, \\
& \delta \tilde{g}_{0 i}=\delta g_{0 i}+a^{2}\left[\xi_{\perp i}^{\prime}+\left(\varsigma^{\prime}-\xi^{0}\right)_{, i}\right], \\
& \delta \tilde{g}_{i j}=\delta g_{i j}+a^{2}\left[2 \frac{a^{\prime}}{a} \delta_{i j} \xi^{0}+2 \varsigma_{, i j}+\left(\xi_{\perp i, j}^{\prime}+\xi_{\perp j, i}^{\prime}\right)\right] .
\end{aligned}
$$

Dessa forma, podemos ver como as perturbações de cada tipo se transformam com uma mudança de coordenadas infinitesimal.

A partir desse resultado e sabendo que os diferentes modos das perturbações da métrica são escritos como (4.5),(4.6) e (4.7), podemos inferir a lei de transformação das funções escalares, vetoriais e tensoriais que descrevem as pertubações.

Para os modos escalares, podemos ver que as variáveis se transformam como:

$$
\begin{gathered}
\tilde{\phi}=\phi-\frac{1}{a}\left(a \xi^{0}\right)^{\prime}, \quad \tilde{B}=B+\varsigma^{\prime}-\xi^{0}, \\
\tilde{\psi}=\psi+\frac{a^{\prime}}{a} \xi^{0}, \quad \tilde{E}=E+\varsigma .
\end{gathered}
$$

Para os modos vetoriais, temos que: 


$$
\tilde{S}_{i}=S_{i}+\xi_{\perp i}^{\prime}, \quad \tilde{F}_{i}=F_{i}+\xi_{\perp i} .
$$

Como vimos anteriormente, os modos perturbativos tensoriais não se alteram com as mudanças de variáveis e assim podemos dizer que as ondas gravitacionais, representadas por $h_{i j}$, são invariantes por transformações do tipo (4.15). Logo, dizemos que são invariantes de calibre.

Dessa forma, vimos que os modos escalares e vetoriais exibem uma ambigüidade de calibre que pode levar ao surgimento de modos perturbativos fictícios. Podemos tratar esse problema de duas maneiras: primeiramente, construindo uma base de variáveis não geométricas que sejam invariantes de calibre, chamadas variáveis de Bardeen [47]; ou fixando um calibre para o problema, o que também elimina a liberdade de calibre.

Se adotarmos uma descrição invariante de calibre, podemos construir combinações lineares das funções escalares ou vetoriais que sejam independentes de $\xi^{\mu}$. As variáveis de Bardeen são definidas como:

- Modos escalares:

$$
\Phi \equiv \phi-\frac{1}{a}\left[a\left(B-E^{\prime}\right)\right]^{\prime}, \quad \Psi \equiv \psi-\frac{a^{\prime}}{a}\left(B-E^{\prime}\right)
$$

- Modos vetoriais:

$$
\bar{v}_{i}=S_{i}-F_{i}^{\prime} .
$$

Podemos ver que nos modos vetoriais, que apresentavam quatro graus de liberdade, somente dois deles caracterizam perturbações físicas, uma vez que os graus de liberdade dependentes de calibre são eliminados com a combinação linear (4.29). Essas variáveis não mudam por uma transformação de calibre e quaisquer combinações delas que sejam invariantes de calibre também serão "variáveis de Bardeen".

Podemos também resolver o problema da ambigüidade de calibre fixando o calibre a ser utilizado. Mesmo em problemas nos quais optamos descrever por meio de variáveis de Bardeen, ou invariantes de calibre, mostra-se conveniente também adotar um calibre específico. Vamos citar aqui dois dos calibres mais conhecidos e utilizados: o calibre longitudinal ou newtoniano e o calibre síncrono.

- Calibre Longitudinal ou Newtoniano: o calibre newtoniano é fixado pelas condições:

$$
B=0, E=0, S_{i}=0,
$$

que deixa o elemento de linha das perturbações da forma: 


$$
d s^{2}=a^{2}(\eta)\left[(1+2 \phi) d \eta^{2}-\left(1-2 \psi-F_{i, j}-F_{j, i}-h_{i j}\right) \delta_{i j} d x^{i} d x^{j}\right] .
$$

Essas escolhas de variáveis fixam completamente o calibre, uma vez que para termos (4.30), obrigatoriamente $\varsigma=\xi^{0}=0$ (e $\left.\xi_{\perp i}^{\prime}=0\right)$. Dessa forma não há uma liberdade de coordenadas e todos os modos perturbativos fictícios são excluídos.

Esse calibre mostra-se muito vantajoso de ser usado em relação aos outros possíveis. Primeiramente, ele fixa totalmente o calibre, eliminando eventuais ambigüidades vindas de modos perturbativos fictícios. Outra vantagem é que, utilizado juntamente com as variáveis de Bardeen em problemas em que as perturbações do TEM para um fluido perfeito com parte espacial, escritas da forma $\delta T_{j}^{i} \propto \delta_{j}^{i}$, reduz as variáveis de Bardeen ao potencial gravitacional newtoniano. Isso deixa o problema com somente uma variável caracterizando o modo escalar das perturbações da métrica. Isso pode ser visto, uma vez que nesse calibre:

$$
\Phi=\phi, \quad \Psi=\psi, \quad \Phi_{i}=E_{i}^{\prime},
$$

em que $\phi$ e $\psi$ são consideradas como potenciais gravitacionais generalizados. Essa é a razão pela qual este calibre também ser denominada de calibre newtoniano ou calibre conforme-newtoniano.

- Calibre Síncrono: o calibre Síncrono é um calibre em que fazemos uma transformação de coordenadas onde $\delta g_{0 \mu}=0$, que são as coordenadas síncronas. Essas coordenadas implicam que:

$$
\phi=0, \quad B=0 .
$$

Esse sistema de coordenadas, apesar de vastamente utilizado na literatura, não fixa o calibre totalmente, uma vez que as condições (4.33) são satisfeitas também em um outro sistema de coordenadas relacionados com o síncrono da forma:

$$
\tilde{\eta}=\eta+\frac{C_{1}\left(x^{j}\right)}{a}, \quad \tilde{x}^{i}=x^{i}+C_{1, i}\left(x^{j}\right) \int \frac{d \eta}{a}+C_{2, i}\left(x^{j}\right)
$$

em que as funções $C_{1}$ e $C_{2}$ são dependentes somente do espaço tridimensional. Dessa forma, permanece uma arbitrariedade nas transformações de coordenadas tridimensionais. Como a coordenada temporal (conforme) está fixada, o nome síncrono aparece então, pois nesse sistema de coordenadas dizemos que todos os relógios em um dado raio de Hubble estão sincronizados. 


\subsubsection{Equações linearizadas para as perturbações cosmológicas}

Definidas as propriedades das perturbações da métrica, podemos agora escrever as equações para as perturbações. Para isso iremos linearizar as equações de Einstein.

Primeiramente, vamos linearizar as equações de Einstein. Para fazê-lo, as perturbações têm que ser pequenas em relação ao fundo e a métrica de fundo tem que ser homogênea e isotrópica. Logo, no caso da métrica de FRW plana (4.4), temos que o tensor de Einstein é escrito da forma:

$$
G_{0}^{0(0)}=\frac{3 \mathcal{H}^{2}}{a^{2}}, \quad G_{i}^{0(0)}=0, \quad G_{j}^{i(0)}=\frac{1}{a^{2}}\left(2 \mathcal{H}^{\prime}+\mathcal{H}^{2}\right) \delta_{j}^{i},
$$

que, pelas equações de Einstein, levam a:

$$
T_{i}^{0(0)}=0, \quad T_{j}^{i(0)} \propto \delta_{j}^{i}
$$

Nesse fundo homogêneo e isotrópico, pequenas perturbações da métrica induzem pequenas perturbações no tensor de Einstein que induzem pequenas perturbações no TEM. Dessa forma, no regime linear, as equações de Einstein são definidas como:

$$
\delta G_{\mu \nu}=8 \pi G \delta T_{\mu \nu}
$$

Como sabemos, a relatividade geral é uma teoria em que há liberdade de calibre e, logo, as perturbações do tensor de Einstein e do TEM também serão dependentes de calibre. Dessa forma, adotaremos o calibre newtoniano para eliminar essa ambigüidade e escreveremos as variáveis do problema em função da variáveis de Bardeen para que as equações não fiquem em função de nenhuma variável dependente de calibre. Para determinarmos a forma das perturbações do tensor de Einstein, temos que utilizar as perturbações da métrica definidas acima. Como as equações de Einstein foram linearizadas, os modos perturbativos escalares, vetoriais e tensoriais são desacoplados e sua evolução pode ser definida separadamente.

- Modos escalares: para determinarmos os modos escalares das perturbações $\delta G_{\mu \nu}$, utilizaremos os modos escalares das perturbações da métrica definidos no elemento de linha (4.10). Assim, as equações de Einstein lineares para as perturbações, em função das variáveis de Bardeen, ficam na forma: 


$$
\begin{aligned}
\delta G_{0}^{0} & =\nabla^{2} \Psi-3 \mathcal{H}\left(\Psi^{\prime}+\mathcal{H} \Phi\right)=4 \pi G a^{2} \delta T_{0}^{0}, \\
\delta G_{i}^{0} & =\left(\Psi^{\prime}+\mathcal{H} \Phi\right)_{, i}=4 \pi G a^{2} \delta T_{i}^{0}, \\
\delta G_{j}^{i} & =\left[\Psi^{\prime \prime}+\mathcal{H}(2+\Phi)^{\prime}+\left(2 \mathcal{H}^{\prime}+\mathcal{H}^{2}\right) \Phi+\frac{1}{2} \nabla^{2}(\Phi-\Psi)\right] \delta_{j}^{i}-\frac{1}{2}(\Phi-\Psi)_{, j}^{, i} \\
& =-4 \pi G a^{2} \delta T_{j}^{i} .
\end{aligned}
$$

- Modos vetoriais: utilizando o elemento de linha dos modos vetoriais das perturbações da métrica (4.12), podemos derivar as equações de Einstein para as perturbações vetoriais:

$$
\begin{aligned}
& \delta G_{i}^{0}=16 \pi G a^{2} \delta T_{i}^{0}, \\
& \delta G_{j}^{i}=\left(\bar{v}_{i, j}+\bar{v}_{j, i}\right)^{\prime}+2 \mathcal{H}\left(\bar{v}_{i, j}+\bar{v}_{j, i}\right)=-16 \pi G a^{2} \delta T_{j(V)}^{i},
\end{aligned}
$$

em que $\delta T_{j(V)}^{i}$ é a parte vetorial da perturbação do TEM.

- Modos tensoriais: para as ondas gravitacionais, podemos escrever as equações de Einstein para as perturbações tensoriais, de (4.14), na forma:

$$
\delta G_{j}^{i}=\left(h_{i j}^{\prime \prime}+2 \mathcal{H} h_{i j}^{\prime}-\nabla^{2} h_{i j}\right)=16 \pi G a^{2} \delta T_{j(T)}^{i}
$$

em que $\delta T_{j(T)}^{i}$ é a parte tensorial das perturbações do TEM, geralmente identicamente nula.

Como citamos anteriormente, $\delta T_{j}^{i} \propto \delta_{j}^{i}$ é diagonal. Contudo, nos modos escalares da equação de Einstein, a parte $(i, j)$ apresenta um termo não diagonal, que precisa ser nulo. Para isso temos que fazer:

$$
\Phi=\Psi
$$

Esse resultado é válido para qualquer conteúdo material com $\delta T_{j}^{i} \propto \delta_{j}^{i}$, ou seja, com conteúdo material que possa ser escrito como um fluido perfeito sem estresse anisotrópico.

Tendo definido as equações de Einstein linearizadas para as perturbações, temos que definir agora o conteúdo material do universo. Como vimos no Capítulo 2, como o universo é homogêneo e isotrópico, podemos escrevê-lo como o TEM de um fluido perfeito (2.15) ou podemos descrevê-lo como preenchido por um tipo diferente de matéria, o campo escalar. Seu TEM é dado por (2.16), que também adota a forma de um fluido perfeito (2.15). Vamos fazer a análise aqui para o campo escalar [48, 49], que é o conteúdo responsável pela aceleração do universo na era inflacionária, como vimos no Capítulo 3 . O estudo das perturbações em função de um campo escalar é importante uma vez que as 
inomogeneidade iniciais postuladas são, na verdade, criadas durante o período inflacionário que as gera devido a flutuações quânticas do campo do inflaton (como veremos na próxima seção). Assim, como estudamos a teoria de perturbação a partir dessas pequenas perturbações do campo do inflaton, é natural estudarmos a evolução das perturbações desse componente. Dessa forma, o campo escalar perturbado é definido como:

$$
\varphi(\eta, \mathbf{x}) \rightarrow \varphi(\eta)+\delta \varphi(\eta, \mathbf{x})
$$

Podemos ver que essa perturbação, quando feita uma transformação de calibre do tipo (4.15), se transforma como:

$$
\delta \tilde{\varphi}=\delta \varphi+\varphi^{\prime} \xi^{0}
$$

e é um modo escalar de perturbação. Como feito para as variáveis perturbativas da métrica, podemos escrever uma quantidade invariante de calibre que descreve a perturbação do campo do inflaton:

$$
\chi \equiv \delta \varphi+\left(B-E^{\prime}\right) \varphi^{\prime} .
$$

Com isso, podemos escrever as perturbações do TEM para as perturbações do inflaton. Para um campo escalar com potencial dado por $V(\varphi)$, no calibre newtoniano, temos que:

$$
\begin{aligned}
& \delta T_{0}^{0}=-\varphi^{\prime} \chi^{\prime}-a^{2} V_{, \varphi} \chi+\Phi \varphi^{\prime}, \\
& \delta T_{i}^{0}=-\partial_{i}\left(\varphi^{\prime} \chi\right), \\
& \delta T_{j}^{i}=-\left(\varphi^{\prime 2} \Phi+a^{2} V_{, \varphi} \chi-\varphi^{\prime} \chi^{\prime}\right) \delta_{j}^{i} .
\end{aligned}
$$

Substituindo essas perturbações do TEM em (4.38-4.40), (4.41-4.42) e (4.43), podemos escrever as equações de Einstein para as perturbações para cada modo.

- Modo escalar: o modo escalar das perturbações é escrito, em cada componente, da forma:

$$
\begin{aligned}
\nabla^{2} \Phi-3 \mathcal{H}\left(\Phi^{\prime}+\mathcal{H} \Phi\right) & =4 \pi G\left(\varphi^{\prime} \chi^{\prime}+a^{2} V_{, \varphi} \chi-\Phi \varphi^{\prime}\right), \\
\Phi^{\prime}+\mathcal{H} \Phi & =4 \pi G \varphi^{\prime} \chi \\
\Phi^{\prime \prime}+3 \mathcal{H} \Phi^{\prime}+\left(\mathcal{H}^{\prime}+2 \mathcal{H}^{2}\right) \Phi & =4 \pi G\left(\varphi^{\prime} \chi^{\prime}-\varphi^{\prime 2} \Phi-a^{2} V_{, \varphi} \chi\right) .
\end{aligned}
$$

Como podemos ver, os modos escalares são os únicos que estão acoplados às perturbações da matéria, no caso do campo do inflaton, e, assim, são os responsáveis pela formação das estruturas no universo. 
Esse sistema de equações apresenta somente duas equações linearmente independentes. Logo, para escrevermos a equação para o modo escalar de perturbação, vamos utilizar (4.52) para determinar $\chi$ e, subtraindo (4.51) de (4.53), podemos escrever:

$$
\Phi^{\prime \prime}+2\left(\mathcal{H}-\frac{\varphi^{\prime \prime}}{\varphi^{\prime}}\right) \Phi^{\prime}-\nabla^{2} \Phi+2\left(\mathcal{H}^{\prime}-\mathcal{H} \frac{\varphi^{\prime \prime}}{\varphi^{\prime}}\right) \Phi=0,
$$

em que utilizamos a equação de Klein Gordon (3.17) em tempo conforme, dada por:

$$
\varphi^{\prime \prime}+2 \mathcal{H} \varphi+a^{2} V_{, \varphi}=0
$$

Essa equação para as perturbações nos fornece a evolução clássica das perturbações cosmológicas. Vamos estudar seu comportamento.

Primeiramente, vamos introduzir as variáveis:

$$
u \equiv \frac{a}{\varphi^{\prime}} \Phi, \quad \theta \equiv \mathcal{H} / a \varphi^{\prime}
$$

que nos fornecem uma equação para as perturbações da forma:

$$
u^{\prime \prime}-\nabla^{2} u-\underbrace{\left(\frac{\theta^{\prime \prime}}{\theta}\right)}_{m_{\text {eff }}^{2}} u=0 .
$$

O termo $\theta^{\prime \prime} / \theta$ funciona como o quadrado de uma "massa" efetiva dependente do tempo.

Para podermos analisar o comportamento dos modos dessas perturbações separadamente, uma vez que a teoria é linear, vamos fazer a transformada de Fourier da variável $u$ :

$$
u(\mathbf{x}, \eta)=\int \frac{d^{3} k}{(2 \pi)^{3 / 2}} u_{\mathbf{k}}(\eta) \mathrm{e}^{i \mathbf{k} \cdot \mathbf{x}},
$$

em que $\mathbf{x}=\mathbf{l} / a$ é a coordenada conforme e $\mathbf{k}=a \mathbf{k}_{\text {fis }}$ é o momento comóvel, que nos fornece a equação:

$$
u_{\mathbf{k}}^{\prime \prime}+\left(k^{2}-\frac{\theta^{\prime \prime}}{\theta}\right) u_{\mathbf{k}}=0
$$

Estudaremos o comportamento assintótico dessa equação. Como vamos comparar a magnitude do momento com a "massa" efetiva, é importante perceber que o inverso da massa, $m_{\text {eff }}^{-1}$ tem magnitude de distância, proporcional ao raio de curvatura do universo [55]. Na época inflacionária, por exemplo, em que o universo está se expandindo exponencialmente, $m_{\text {eff }}^{-1} \approx(a H)^{-1}$, sendo que $H$ é aproximadamente constante e os estados assintóticos então serão comparados com o tamanho do horizonte.

\section{$\underline{1^{\circ} \mathrm{Caso}}$}


Para perturbações de comprimento de onda pequeno, ou seja, $k^{2} \gg \theta^{\prime \prime} / \theta$, a solução de (4.59) é uma onda plana, dada por:

$$
u_{\mathbf{k}}(\eta)=A_{\mathbf{k}}^{+} \mathrm{e}^{i k \eta}+A_{\mathbf{k}}^{-} \mathrm{e}^{-i k \eta},
$$

em que $A_{\mathrm{k}}^{ \pm}$são constantes de integração. Essa solução nos fornece a equação para as pertubações invariantes de calibre, em tempo próprio, que também pode ser escrito como:

$$
\Phi_{\mathbf{k}}(t, \mathbf{x}) \simeq \dot{\varphi} \mathrm{e}^{i k \mathbf{x}}\left[A_{\mathbf{k}}^{+} \operatorname{sen}\left(k \int \frac{d t}{a}\right)+A_{\mathbf{k}}^{-} \cos \left(k \int \frac{d t}{a}\right)\right]
$$

Essas soluções representam perturbações que oscilam com amplitude variável com o tempo. Como nesse estado o termo $\theta^{\prime \prime} / \theta$ é desprezado, isso significa que, apesar das perturbações sentirem a expansão do universo, elas não sentem a curvatura do espaçotempo. Porém, os comprimentos de onda físicos são esticados com a expansão do universo. Eles podem ser esticados até ficarem maiores que o horizonte se as perturbações forem esticadas mais rápido do que a velocidade com que o raio de curvatura do universo cresce. Vamos ver que no período inflacionário esse mecanismo de fato ocorre, com os modos cruzando o horizonte com uma taxa exponencial.

$\underline{2^{\circ} \text { Caso }}$

Para comprimentos de onda grandes, com $k^{2} \ll \theta^{\prime \prime} / \theta$, temos a equação para as perturbações:

$$
\theta u_{\mathbf{k}}^{\prime \prime}-\theta^{\prime \prime} u_{\mathbf{k}}=0
$$

que pode ser resolvida pelo método do wronskiano [50, 51]. Tendo a primeira solução trivial da forma $u_{1, \mathbf{k}}=C_{1} \theta$, podemos calcular a segunda solução através do wronskiano:

$$
W=u_{1, \mathbf{k}} u_{2, \mathbf{k}}^{\prime}-u_{1, \mathbf{k}}^{\prime} u_{2, \mathbf{k}}=u_{1, \mathbf{k}}^{2} \frac{d}{d \eta}\left(\frac{u_{2, \mathbf{k}}}{u_{1, \mathbf{k}}}\right)(=\text { const. }) \text {. }
$$

Assim, como temos uma equação da forma:

$$
u_{\mathbf{k}}^{\prime \prime}+Q(\eta) u_{\mathbf{k}}=0
$$

integramos o wronskiano para obter a solução geral que é da forma: 


$$
\begin{aligned}
u_{\mathbf{k}} & \simeq C_{1} \theta+C_{2} \theta \int_{\eta_{i}} \frac{d \eta}{\theta^{2}}, \\
& =\frac{A}{\varphi^{\prime}}\left[\frac{1}{a} \int_{\eta_{i}} a^{2}(\eta) d \eta\right]^{\prime},
\end{aligned}
$$

em que $C_{1}, C_{2}$ e $A$ são constantes de integração. Essa solução nos fornece as equações para as perturbações invariantes de calibre:

$$
\Phi(t, \mathbf{x}) \simeq A\left(1-\frac{H}{a} \int a d t\right)
$$

Como podemos ver, a perturbação da métrica nos fornece duas soluções fundamentais. Vamos analisar seus comportamentos para o caso específico em que o universo se expande como uma lei de potência, $a \sim t^{p} \operatorname{com} p>0(p \neq 1)$. Nesse caso, a equação (4.67) das perturbações com comprimentos de onda grande apresenta um termo constante e um termo decrescente:

$$
\Phi_{l p}(t, \mathbf{x})=A^{(1)}\left(1-\frac{p}{p+1}\right)+A^{(2)} \frac{1}{t^{1+p}} .
$$

Dessa forma, o termo constante é dominante para $p>1$ (expansão acelerada) e para $p<1$ (expansão desacelerada). Essa solução constante nos fornece a seguinte interpretação: que as perturbações com comprimento de onda grande (fora do horizonte) estão congeladas e não crescem nem decrescem enquanto continuarem maiores que o horizonte. Assim, podemos pensar que, quando essas perturbações reentrarem no horizonte, elas serão constantes, independentemente de quando saíram do horizonte.

Para o caso geral, essa análise é um pouco mais complicada. Para isso, vamos reescrever nossas equações em termos de novas variáveis.

Para podermos interpretar de maneira mais conveniente a evolução das perturbações de comprimento de onda grande, vamos construir uma variável invariante de calibre que é conservada nessas escalas ${ }^{1}[30]$ :

$$
\mathcal{R} \equiv \Phi+\frac{2 \mathcal{H}}{8 \pi G} \frac{1}{\varphi^{\prime 2}}\left(\Phi^{\prime}+\mathcal{H} \Phi\right)
$$

Essa variável é conhecida como a perturbação da curvatura comóvel e foi introduzida e utilizada em $[47,56,57,49]$. Ela representa o potencial gravitacional em superfícies comóveis com $\delta \phi=0$ [60]. A equação (4.54), em termos dessa variável, é dada por:

\footnotetext{
${ }^{1}$ Em um universo plano.
} 


$$
\mathcal{R}^{\prime}=\frac{2 \mathcal{H}}{8 \pi G} \frac{1}{\varphi^{\prime 2}} \nabla^{2} \Phi
$$

Essa variável é dita conservada em perturbações com comprimentos de onda grandes uma vez que nesse estado assintótico o termo $\nabla^{2} \Phi$ é desprezível.

Outra variável invariante de calibre também muito utilizada é [30]:

$$
\zeta \equiv \Phi+\frac{2}{3} \frac{H^{-1} \dot{\Phi}+\Phi}{1+\omega}
$$

em que $\omega=p / \rho$. Essa variável foi primeiramente introduzida por Bardeen, Steinhardt e Turner [52]. Ela representa a perturbação da curvatura numa hipersuperfície de densidade uniforme e a equação para as perturbações (4.54) em termos dessa nova variável é dada, em tempo próprio, por:

$$
\frac{3}{2} \dot{\zeta}(1+\omega)=\ddot{\Phi}+\left(H-2 \frac{\ddot{\varphi}}{\dot{\varphi}}\right) \dot{\Phi}+2\left(\dot{H}-H \frac{\ddot{\varphi}}{\dot{\varphi}}\right) \Phi
$$

em que o termo proporcional a $\nabla^{2} \Phi$ é desprezado no regime de grandes comprimentos de onda. Nesse regime, essa equação se reduz a:

$$
\dot{\zeta}(1+\omega)=0
$$

em que podemos ver que $\zeta$ é constante (se conserva) para grandes comprimentos de onda com qualquer equação de estado quando temos somente um campo de matéria [58, 59].

O fato de $\mathcal{R}^{\prime}=0$ e $\dot{\zeta}=0$, ou seja, $\mathcal{R}$ e $\zeta$ serem contantes em escalas de grandes comprimentos de onda, implica que $\Phi=$ const., como podemos ver a partir das definições dessas curvaturas. Ou seja, a perturbação nessas escalas é constante e permanece assim enquanto permanecer nessas escalas.

Essas duas variáveis coincidem no regime inflacionário com condições de rolagem lenta e se conservam para comprimentos de onda maiores que o horizonte de Hubble. Portanto, podemos dizer que perturbações maiores que o horizonte de Hubble são constantes ou são congeladas e não evoluem enquanto permanecem nessa escala. Essa característica é importante, uma vez que relaciona as flutuações iniciais e finais no período inflacionário, sendo que o espectro de potências é calculado em função da variável $\mathcal{R}$. Como visto acima, o fato dessas perturbações serem constantes mostra que quando elas reentrarem no horizonte seu espectro será constante. Esse fato é observado experimentalmente [24] e é um dos resultados mais promissores e que corrobora a teoria inflacionária. Veremos com mais detalhe o espectro das flutuações na seção seguinte.

Dessa discussão sobre as perturbações escalares, podemos sumarizar que, se o universo passa por um estágio de expansão acelerada como a inflação, o parâmetro de Hubble varia muito lentamente, podendo ser considerado quase constante, enquanto o fator de 
escala varia quase-exponencialmente. Os modos perturbativos que estão inicialmente com comprimento de onda menor que o horizonte de Hubble, são rapidamente esticados e deixam o horizonte de Hubble, onde se mantêm constantes ${ }^{2}$. Esse efeito da inflação faz com que essas amplitudes congeladas sejam sempre as mesmas, a do cruzamento do horizonte, independente de sua escala, o que fornece um espectro invariante de escala para as perturbações.

- Modo vetorial: como pudemos ver acima, todas as perturbações do inflaton eram escalares. Os modos vetoriais de perturbação das equações de Einstein são dados por:

$$
\begin{aligned}
\nabla^{2} \Phi_{i} & =0, \\
\Phi_{i}^{\prime}+2 \mathcal{H} \Phi_{i} & =0 .
\end{aligned}
$$

É fácil ver que, de (4.75):

$$
\Phi_{i}(\eta, \mathbf{x})=C_{i}(\mathbf{x}) a^{-2}
$$

que representa uma solução que decai rapidamente com o aumento do fator de escala, ou seja, em um universo que está se expandindo. Assim, somente a partir dessa equação já podemos ver que, para que os modos vetoriais de perturbações sejam de importância cosmológica, seus modos vetoriais seminais teriam que ter comprimento de onda muito grandes. Como vimos nesse capítulo, se a "semente" das perturbações é gerada na inflação, a teoria da inflação impede que essas perturbações sejam grandes o suficiente para serem detectadas atualmente.

Os modos vetoriais não contribuem para o colapso gravitacional e para a formação de estruturas e representam movimentos rotacionais do fluido cósmico.

- Modo tensorial: assim como para os modos vetoriais, na teoria linear o modo tensorial não contém perturbações do inflaton (ou seja, do conteúdo material) e também não participa da formação de estruturas do universo. Dessa forma, as ondas gravitacionais evoluem como:

$$
h_{i j}^{\prime \prime}+2 \mathcal{H} h_{i j}^{\prime}-\nabla^{2} h_{i j}=0,
$$

que tem a forma da equação de um oscilador harmônico amortecido. Apesar de não contribuírem para a formação das estruturas, o estudo das ondas gravitacionais é de grande importância. Não faremos uma análise muito extensa desse modo perturbativo nessa dissertação, mas mostraremos suas principais características.

\footnotetext{
${ }^{2}$ Essa amplitude cresce muito pouco fora do horizonte de Hubble, o que gera pequenos desvios no espectro plano da inflação. Veremos mais sobre isso na próxima seção.
} 
Podemos expandir $h_{i j}$ da seguinte forma:

$$
h_{i j}=\frac{1}{a(\eta)} \int \frac{d^{3} k}{(2 \pi)^{3}}\left[\mu_{\mathbf{k}}(\eta) \epsilon_{i j}(\mathbf{k}) e^{i \mathbf{k x}}+\text { c.c. }\right]
$$

e inserido em (4.77) nos fornece a equação para $\mu_{\mathbf{k}}(\eta)$ :

$$
\mu_{\mathbf{k}}^{\prime \prime}+\left(k^{2}-\frac{a^{\prime \prime}}{a}\right) \mu_{\mathbf{k}}=0
$$

Essa equação é a equação de um oscilador harmônico paramétrico e é semelhante à equação (4.59) para as perturbações escalares. Lembrando que o tensor $h_{i j}$ foi definido como sendo transverso e tendo traço nulo, em (4.8), temos que o elemento tensorial da expansão (4.78) terá que ser simétrico e transverso e com traço nulo:

$$
\epsilon_{i j}=\epsilon_{j i}, \epsilon_{j}^{i}=\epsilon_{i j} k^{i}=0
$$

\subsection{Teoria Quântica das Perturbações Cosmológicas: flutuações quânticas e o "nascimento" das pertur- bações cosmológicas}

A teoria da inflação foi formulada inicialmente com o intuito de resolver os problemas das condições iniciais do MCP. Contudo, essa teoria apresenta como conseqüência a explicação para a formação das pequenas flutuações responsáveis por formar as estruturas não-lineares do universo e seu espectro. A teoria das perturbações cosmológicas clássicas estudada acima tem como condição inicial, ou postuladas, as pequenas perturbações iniciais. A teoria da inflação fornece naturalmente um mecanismo para a formação dessas perturbações iniciais. Esse mecanismo é considerado um tema central para a cosmologia moderna e representa uma das descobertas de maior importância da teoria inflacionária.

A inflação prevê a criação das perturbações primordiais via flutuações quânticas que são esticadas até escalas cosmológicas durante o período inflacionário. Como vimos anteriormente, mesmo perturbações que estavam fora do horizonte de Hubble após o período inflacionário, estiveram dentro do horizonte no começo da inflação. Essas perturbações inicialmente se comportam como ondas planas e oscilam mais rapidamente. Assim, suas flutuações quânticas se comportam efetivamente como ondas planas em um espaço-tempo de Minkowski.

Para entendermos e descrevermos a formação das perturbações é necessário utilizarmos a relatividade geral e a mecânica quântica, uma vez que queremos descrever a criação de perturbações na forma de partículas de um estado de vácuo que gravita, no caso, se expande, conforme a relatividade geral. Partimos, portanto, de um universo composto por 
uma componente de campo escalar, no caso um condensado de campo escalar, sem partículas. Vamos quantizar as perturbações cosmológicas desse condensado e as perturbações de primeira ordem da métrica em relação ao fundo homogêneo e isotrópico.

Partimos da ação de Einstein-Hilbert [49], que descreve esse sistema do campo de matéria acoplado minimamente com o campo gravitacional, da forma:

$$
\begin{aligned}
S & =\frac{1}{2} \int d^{4} x\left(\mathcal{L}_{\text {grav }}+\mathcal{L}_{m}\right) \\
& =\frac{1}{16 \pi G} \int d^{4} x \sqrt{-g}\left(R+\mathcal{L}_{m}^{\prime}\right)
\end{aligned}
$$

em que a Lagrangeana da gravitação é representada pelo escalar de Ricci, $R$, e a Lagrangeana da matéria é composta por um campo escalar representada em (3.13).

Queremos quantizar esse sistema. Contudo, para faze-lo é necessário encontrarmos as variáveis de quantização canônica corretas. Elas nos fornecerão um procedimento de quantização consistente, assim como serão importantes para a obtenção dos observáveis corretos da teoria. Elas nos fornecerão uma ação quadrática efetiva, na forma de osciladores harmônicos para quantizarmos e vai permitir que entendamos melhor o vácuo da teoria, que nem sempre pode ser definido em espaços curvos arbitrários. Como vimos pelo estudo das perturbações cosmológicas clássicas na relatividade geral, a descrição dessa teoria é possível ser feita somente em função de uma variável. Queremos encontrar, então, a variável canônica que descreve essas perturbações, e que será nossa variável a ser quantizada. Para isso iremos expandir essa ação, como em uma expansão de Taylor, até segunda ordem. Esse cálculo é muito extenso, principalmente por causa dos problemas de dependência de calibre que a relatividade geral apresenta. Para isso utilizaremos o formalismo ADM [61].

Esse formalismo perturbativo foi desenvolvido por R. Arnowitt, S. Deser e C. W. Misner e simplifica enormemente a obtenção de uma forma canônica para a ação de EinsteinHilbert para as perturbações. Desenvolvido para tratar da quantização de sistemas em espaço-tempo curvos, ou em fundos gravitacionais, esse formalismo hamiltoniano da relatividade geral é capaz de reduzir a ação (4.82) para uma ação efetiva de um campo em um espaço-tempo de Minkowski sob a influência de um potencial, separando os graus de liberdade físicos dos não-físicos. Convém ressaltar aqui que esse método é perturbativo. No Capítulo 7, mostraremos, de forma não perturbativa, que a ação do campo escalar em espaços-tempo curvos é equivalente à ação quadrática de um campo em um espaço-tempo de Minkowski sob a influência de um potencial gravitacional.

Escrevendo a métrica na forma de uma métrica ADM (parametrização):

$$
d s^{2}=\left(\mathcal{N}^{2}-\mathcal{N}_{i} \mathcal{N}^{i}\right) d t^{2}-2 \mathcal{N}_{i} d x^{i} d t-g_{i j} d x^{i} d x^{j}
$$


em que $\mathcal{N}$ e $\mathcal{N}_{i}$ são a função lapso e o vetor de deslocamento e $g_{i j}$ é a métrica do espaçotempo em uma superfície de tempo constante. Substituindo essa métrica na ação de Einstein-Hilbert (4.82), podemos obter:

$$
\begin{aligned}
S & =\frac{1}{16 \pi G} \int d^{4} x \sqrt{-g}\left[\mathcal{N} R^{(3)}+\mathcal{N}\left(K_{i j} K^{i j}-K^{2}\right)-8 \pi G \mathcal{N}\left(g^{i j} \partial_{i} \varphi \partial_{j} \varphi-2 V(\varphi)\right)\right. \\
& +8 \pi G \mathcal{N}^{-1}\left(\dot{\varphi}-\mathcal{N}^{i} \partial_{i} \varphi_{i}\right)
\end{aligned}
$$

em que $R^{(3)}$ é o escalar de Ricci em relação à métrica $g_{i j}$ e $K_{i j}$ é o tensor de curvatura extrínseco da superfície de tempo constante dado por:

$$
K_{i j}=\frac{1}{2} N^{-1}\left(\nabla_{i} N_{j}+\nabla_{j} N_{i}-\dot{g}_{i j}\right)
$$

e $K=K_{i}^{i}=g_{i j} K^{j}$. A partir dessa nova ação, podemos determinar as equações de EulerLagrange das variáveis $\mathcal{N}$ e $\mathcal{N}_{i}$, variando a ação em função dessas variáveis. Dessa forma, as equações de movimento são:

$$
\begin{array}{r}
R^{(3)}-\left(K_{i j} K^{i j}-K^{2}\right)-2 V(\varphi)-8 \pi G g^{i j} \partial_{i} \varphi \partial_{j} \varphi+8 \pi G \mathcal{N}^{-2}\left(\dot{\varphi}-\mathcal{N}^{i} \partial_{i} \varphi_{i}\right)^{2}=0 \\
\nabla_{j}\left(K_{i}^{j}-K \delta_{i}^{j}\right)-8 \pi G \mathcal{N}^{-1}\left(\dot{\varphi}-\mathcal{N}^{i} \partial_{i} \varphi_{i}\right) \partial_{i} \varphi=0
\end{array}
$$

Com isso, podemos comparar a métrica (4.83) com a métrica das perturbações (4.31) que calculamos no calibre newtoniano para podermos começar a relacionar essas variáveis com as variáveis que conhecemos. Podemos identificar:

$$
N^{2}=(1+2 \Phi), \quad N_{i}=0, \quad g_{i j}=a^{2}\left[\delta_{i j}(1-2 \Psi)+F_{i, j}+F_{j, i}+2 h_{i j}\right] .
$$

Feita a associação, podemos reescrever as equações (4.86-4.87) em função das variáveis da métrica em primeira ordem. Dessa forma, essas equações representam os vínculos do sistema. Podemos identificar nesse sistema de equações que temos perturbações escalares, vetoriais e tensoriais. Contudo, vamos analisar somente as escalares e tensoriais, uma vez que as vetoriais decaem rapidamente, como vimos.

Agora queremos expandir nossa ação. Substituindo as variáveis de (4.88) na ação (4.84) e levando em conta os vínculos e as equações de fundo como as equações de Friedmann e de Klein Gordon, podemos expandir essa ação até segunda ordem. Essa cálculo é bem extenso e pode ser visto em partes em [49]. Os termos de ordem zero da expansão nos fornecem as equações para o fundo homogêneo e isotrópico e obviamente não são de interesse para avaliarmos as perturbações. Os termos de primeira ordem da expansão são proporcionais às equações do fundo e, assim, são iguais a zero. Somente sobram, 
então, os termos de segunda ordem na expansão, que fornecem a ação quadrática para as perturbações. Como mencionamos acima, da análise da evolução das perturbações clássicas, vemos que somente uma variável dinâmica descreve as perturbações de cada tipo, seja escalar ou tensorial. Definindo então as variáveis chamadas de variáveis de Mukhanov-Sasaki:

$$
v \equiv z \mathcal{R}
$$

com $z=1 / \theta=a \varphi^{\prime} / \mathcal{H}$ e $\mathcal{R}$, a perturbação de curvatura, representando o modo escalar das perturbações. Representando as perturbações tensoriais, temos:

$$
\mu_{i j} \equiv \sqrt{\frac{M_{p}^{2}}{4}} a h_{i j}
$$

Como o tensor $\mu_{i j}$ admite a decomposição (4.78), temos que, do tensor de polarização, $h_{i j}$ pode ser decomposto em duas polarizações:

$$
h_{i j}(\eta, \mathbf{x})=h_{+}(\eta, \mathbf{x}) \epsilon_{i j}^{+}+h_{\times}(\eta, \mathbf{x}) \epsilon_{i j}^{\times} .
$$

Em termos dessas variáveis canônicas e invariantes de calibre, a ação quadrática fica na forma:

$$
\begin{aligned}
S^{(2)} & =\frac{1}{2} \int d \eta d^{3} x\left(\mathcal{L}_{\text {esc }}+\mathcal{L}_{\text {tens }}\right) \\
& =\frac{1}{2} \int d \eta d^{3} x\left[\left(v^{\prime}\right)^{2}-v_{, i} v_{, i}+\frac{z^{\prime \prime}}{z} v^{2}\right] \\
& +\frac{1}{2} \int d \eta d^{3} x \sum_{\lambda=+, \times}\left[\left(\mu_{\lambda}^{\prime}\right)^{2}-\mu_{\lambda, i} \mu_{\lambda, i}+\frac{a^{\prime \prime}}{a} \mu_{\lambda}^{2}\right] .
\end{aligned}
$$

Podemos ver que partimos de uma ação de um campo escalar com potencial $V(\varphi)$ em um espaço-tempo curvo para uma ação que representa campos (variáveis canônicas) em um espaço-tempo de Minkowski com termos de "massa", $\frac{z^{\prime \prime}}{z}$ e $\frac{a^{\prime \prime}}{a}$, dependentes do tempo, como se um campo externo estivesse presente. Veremos no Capítulo 7 que essa possibilidade de escrever a ação de Einstein-Hilbert dessa forma, análoga à ação de um campo em presença de um campo externo em um espaço-tempo de Minkowski, será importante para podermos fazer a analogia entre a produção de partículas na Inflação com o efeito Schwinger, um dos temas centrais dessa dissertação.

Podemos obter, então, as equações de Euler-Lagrange variando a ação em função de cada uma das variáveis de Mukhanov-Sasaki: 


$$
v^{\prime \prime}-\nabla^{2} v-\frac{z^{\prime \prime}}{z} v=0, \quad \mu_{\lambda}^{\prime \prime}-\nabla^{2} \mu_{\lambda}-\frac{a^{\prime \prime}}{a} \mu_{\lambda}=0
$$

ou, se fizermos uma transformada de Fourier, que desacopla os modos:

$$
v_{\mathbf{k}}^{\prime \prime}+\left(k^{2}-\frac{z^{\prime \prime}}{z}\right) v_{\mathbf{k}}=0, \quad \mu_{\mathbf{k}}^{\prime \prime}+\left(k^{2}-\frac{a^{\prime \prime}}{a}\right) \mu_{\mathbf{k}}=0 .
$$

Essas equações são análogas às obtidas em (4.59) e (4.79) e a evolução dessas variáveis segue a mesma dinâmica descrita na seção anterior.

É interessante também formular o problema no formalismo Hamiltoniano, que nos fornece uma visão diferente do sistema, uma vez que, ao invés de recair em equações de segundo grau como no formalismo Lagrangeano, fornece equações de primeiro grau, as equações de Hamilton definidas no espaço de fase. Para isso vamos determinar o momento canônico conjugado das variáveis de Mukhanov-Sasaki:

$$
\begin{aligned}
& \Pi_{v}(\eta, \mathbf{x})=\frac{\partial \mathcal{L}^{(2)}}{\partial v^{\prime}}=v^{\prime}, \\
& \Pi_{\mu}(\eta, \mathbf{x})=\frac{\partial \mathcal{L}^{(2)}}{\partial \mu_{\lambda}^{\prime}}=\mu_{\lambda}^{\prime},
\end{aligned}
$$

que nos fornece a Hamiltoniana para o sistema, por uma transformação de Legendre:

$$
\begin{aligned}
H & =\int d^{3} x\left(v^{\prime} \Pi_{v}+\mu_{\lambda}^{\prime} \Pi_{\mu}-\mathcal{L}^{(2)}\right) \\
& =\frac{1}{2} \int d^{3} x\left[\Pi_{v}^{2}+v_{, i} v_{, i}-\frac{z^{\prime \prime}}{z} v^{2}\right]+\frac{1}{2} \int d^{3} x\left[\Pi_{\mu}^{2}+\mu_{\lambda, i} \mu_{\lambda, i}-\frac{a^{\prime \prime}}{a} \mu_{\lambda}^{2}\right] .
\end{aligned}
$$

A dinâmica das variáveis de campo é dada pelas equações de Hamilton, definidas como:

$$
\begin{array}{cc}
i v^{\prime}=[v, H], & i \Pi_{v}^{\prime}=\left[\Pi_{v}, H\right], \\
i \mu_{\lambda}^{\prime}=\left[\mu_{\lambda}, H\right], & i \Pi_{\mu}^{\prime}=\left[\Pi_{\mu}, H\right] .
\end{array}
$$

\subsubsection{Quantização}

Com a ação quadrática em mãos, podemos passar para o procedimento de quantização canônica do sistema. Vamos apresentar aqui o procedimento de quantização canônica de um campo escalar, no caso real, em presença de um campo externo gravitacional. Esse 
procedimento é padrão e será introduzido aqui e utilizado em outros pontos da dissertação. Dessa forma, o procedimento de quantização canônica para os modos escalares e tensoriais é o mesmo e será feito para uma variável generalizada qualquer $Q_{l}(\eta, \mathbf{x})$ e podendo ser aplicado para cada um dos modos $l$, que representam os modos escalares e tensoriais, modificando os termos necessários no final.

O funcional de ação que representa a ação quadrática das perturbações é dado, em função da variável generalizada, por:

$$
S\left[Q_{l}\right]=\int d \eta d^{3} x \underbrace{\left[\left(Q_{l}^{\prime}\right)^{2}-Q_{l, i} Q_{l}^{i}+\frac{Z_{l}^{\prime \prime}}{Z_{l}} Q_{l}^{2}\right]}_{\mathcal{L}^{(2)}},
$$

em que $Z_{l}$ é o representa o termo de "massa" para cada modo, sendo igual a $z$ para o modo escalar e igual a a no caso tensorial. As equações de movimento, ou de Euler-Lagrange, são definidas como sendo:

$$
\frac{\partial \mathcal{L}}{\partial Q_{l}}-\partial_{\mu}\left[\frac{\partial \mathcal{L}}{\partial\left(\partial_{\mu} Q_{l}\right)}\right]=0 .
$$

As equações de movimento (4.93) estão sintetizadas na equação de movimento para (4.101):

$$
Q_{l}^{\prime \prime}+\left(\nabla^{2}-\frac{Z_{l}^{\prime \prime}}{Z_{l}}\right) Q_{l}=0 .
$$

Fazendo a quantização canônica, nossas variáveis canônicas se tornam operadores quânticos:

$$
\begin{cases}Q_{l} & \rightarrow \hat{Q}_{l} \\ \Pi_{l} & \rightarrow \hat{\Pi}_{l}\end{cases}
$$

que devem obedecer às relações de comutação:

$$
\begin{aligned}
{\left[\hat{Q}_{l}(\eta, \mathbf{x}), \hat{\Pi}_{l^{\prime}}(\eta, \mathbf{y})\right] } & =i \delta_{l l^{\prime}} \delta(\mathbf{x}-\mathbf{y}), \\
{\left[\hat{Q}_{l}(\eta, \mathbf{x}), \hat{Q}_{l^{\prime}}(\eta, \mathbf{y})\right]=\left[\hat{\Pi}_{l}(\eta, \mathbf{x}), \hat{\Pi}_{l^{\prime}}(\eta, \mathbf{y})\right] } & =0 .
\end{aligned}
$$

Podemos definir o mesmo procedimento para as variáveis na decomposição de Fourier, sendo que $\left[\hat{Q}_{\mathbf{k}, l}(\eta), \hat{\Pi}_{\mathbf{k}^{\prime}, l^{\prime}}(\eta)\right]=\delta_{l l^{\prime}} \delta\left(\mathbf{k}-\mathbf{k}^{\prime}\right)$.

Uma solução geral das equações (4.93) pode ser escrita como uma decomposição:

$$
\hat{Q}_{l}(\eta, \mathbf{x})=\int \frac{d^{3} k}{(2 \pi)^{3 / 2}}\left[Q_{\mathbf{k}, l}(\eta) \hat{a}_{\mathbf{k}, l} \mathrm{e}^{-i \mathbf{k x}}+Q_{\mathbf{k}, l}^{*}(\eta) \hat{a}_{\mathbf{k}, l}^{\dagger} e^{i \mathbf{k x}}\right]
$$


em que $\hat{a}_{\mathbf{k}, l}$ e $\hat{a}_{\mathbf{k}, l}^{\dagger}$ são respectivamente os operadores de aniquilação e criação de partículas agindo no vácuo da representação de Fock. Com a introdução desses operadores, o status de operador passa do campo para eles. Esse procedimento é conhecido como segunda quantização, em que a variável de campo se torna um operador que age nos estados quânticos de partículas. As regras de comutação das variáveis de campo são traduzidas para esses operadores, de forma que eles obedecem às regras de comutação em tempos iguais:

$$
\begin{aligned}
& {\left[\hat{a}_{\mathbf{k}, l}, \hat{a}_{\mathbf{k}^{\prime}, l^{\prime}}^{\dagger}\right]=\delta_{l l^{\prime}} \delta\left(\mathbf{k}-\mathbf{k}^{\prime}\right), } \\
& {\left[\hat{a}_{\mathbf{k}, l}, \hat{a}_{\mathbf{k}^{\prime}, l^{\prime}}\right]=\left[\hat{a}_{\mathbf{k}, l}^{\dagger}, \hat{a}_{\mathbf{k}^{\prime}, l^{\prime}}^{\dagger}\right]=0 . }
\end{aligned}
$$

Essas regras de comutação somente são válidas se os modos $Q_{\mathbf{k}, l}(\eta)$ obedecerem à relação de normalização, vinda da relação de comutação (4.105):

$$
Q_{\mathbf{k}, l}^{\prime} Q_{\mathbf{k}, l}^{*}-Q_{\mathbf{k}, l}^{*^{\prime}} Q_{\mathbf{k}, l}=2 i
$$

que representa o wronskiano das soluções clássicas. Essa normalização nos permite fixar a amplitude de $Q_{\mathbf{k}, l}(\eta)$ que é compatível com o princípio de incerteza de Heisenberg. Podemos ver que essas variáveis satisfazem:

$$
Q_{\mathbf{k}, l}^{\prime \prime}+\underbrace{\left(k^{2}-\frac{Z_{l}^{\prime \prime}}{Z_{l}}\right)}_{\omega_{\mathbf{k}, l}^{2}(\eta)} Q_{\mathbf{k}, l}=0 .
$$

O operador Hamiltoniano do sistema, em função desses operadores de criação e aniquilação, pode ser escrito da forma:

$$
\hat{H}_{\mathbf{k}}=\omega_{\mathbf{k}, l}^{2}(\eta) \hat{a}_{\mathbf{k}}^{\dagger} \hat{a}_{\mathbf{k}}
$$

em que $\omega_{\mathbf{k}, l}(\eta)$ é a freqüência.

O próximo passo da quantização canônica é definir o espaço de Fock no qual as variáveis agem. Sabe-se que a Hamiltoniana tem auto-estados com autovalores dados pela energia do sistema. Como a Hamiltoniana é quadrática e portanto positiva definida, sabemos que deve existir um estado com energia mais baixa, $|0\rangle$, tal que $H|0\rangle=E_{0}|0\rangle$. Logo, para um campo escalar, podemos definir um único estado de vácuo, como sendo:

$$
\hat{a}_{\mathbf{k}, l}|0\rangle=0, \hat{b}_{\mathbf{k}, l}|0\rangle=0, \forall \mathbf{k} .
$$

ou seja, o estado que não possui partículas (ou antipartículas). Assim, o operador $\hat{a}_{\mathbf{k}}$ 
é chamado de operador de aniquilação de partículas e $\hat{a}_{\mathbf{k}, l}^{\dagger}$ de operadores de criação de partículas. Na descrição de Heisenberg, ou seja, em que os operadores é que carregam a evolução temporal, os estados quânticos geram um espaço de Hilbert. O espaço de Hilbert nos quais esses operadores agem é denominado espaço de Fock. Os operadores de criação criam um estado que contém partículas e antipartículas, respectivamente:

$$
\hat{a}_{\mathbf{k}, l}^{\dagger}|0\rangle=|1 \mathbf{k}, l\rangle, \hat{b}_{\mathbf{k}, l}|0\rangle=|1 \mathbf{k}, l\rangle
$$

e todos os outros estados do sistema podem ser construídos a partir desse operador de criação.

Apesar do procedimento de quantização ser muito semelhante ao dos campos livres, no sistema em presença de um campo externo a invariância translacional no espaço e no tempo é quebrada e, assim, não podemos definir uma base ortonormal única. Ou seja, o estado de vácuo definido em (4.113) é agora dependente da base em que a quantização foi feita e tem direção temporal definida. Em particular, operadores associados com o operador de aniquilação têm freqüência positiva e os associados com o operador de criação têm freqüência negativa.

Se considerarmos o valor esperado de um operador bilinear $\hat{N}_{\mathbf{k}}=\hat{a}_{\mathbf{k}}^{\dagger} \hat{a}_{\mathbf{k}}$ no vácuo e em um estado de muitas partículas, temos que:

$$
\left\langle 0\left|\hat{N}_{\mathbf{k}}(\eta)\right| 0\right\rangle=0, \quad\left\langle n\left|\hat{N}_{\mathbf{k}}(\eta)\right| n\right\rangle=n_{\mathbf{k}}, \forall \mathbf{k}
$$

Logo, o valor esperado do operador bilinear $\hat{N}_{\mathbf{k}}$ é chamado de operador número e fornece o número de partículas com momento $\mathbf{k}$ e o estado $|n\rangle$ é um auto estado desse operador.

O valor do operador número em um tempo inicial $\eta_{1}$ pode ser igual a zero para o estado de vácuo $\left|0, \eta_{1}\right\rangle$, uma vez que $\hat{a}_{\mathbf{k}, l}\left(\eta_{1}\right)\left|0, \eta_{1}\right\rangle=0$, mas diferente de zero para um instante posterior $\eta_{2}$ :

$$
\left\langle 0, \eta_{2}\left|\hat{N}_{\mathbf{k}}\left(\eta_{1}\right)\right| 0, \eta_{2}\right\rangle \neq 0
$$

Dessa forma, os operadores de criação e aniquilação em diferentes tempos se misturam e a Hamiltoniana não é quadrática nesse caso. Podemos escrever os modos de freqüência positiva e negativa, ou os operadores de criação e aniquilação, para um tempo $\eta_{2} \mathrm{em}$ função dos operadores em $\eta_{1}$ por meio de uma transformada de Bogoliubov:

$$
\left(\begin{array}{c}
\hat{a}_{\mathbf{k}, l}\left(\eta_{2}\right) \\
\hat{a}_{\mathbf{k}, l}^{\dagger}\left(\eta_{2}\right)
\end{array}\right)=\left(\begin{array}{cc}
\alpha_{k} & \beta_{k}^{*} \\
\beta_{k} & \alpha_{k}^{*}
\end{array}\right)\left(\begin{array}{c}
\hat{a}_{\mathbf{k}, l}\left(\eta_{1}\right) \\
\hat{a}_{\mathbf{k}, l}^{\dagger}\left(\eta_{1}\right)
\end{array}\right),
$$

em que os coeficientes de Bogoliubov $\alpha_{k}$ e $\beta_{k}$, das relações de comutação (4.108-4.109), obedecem a: 


$$
\left|\alpha_{k}\right|^{2}-\left|\beta_{k}\right|^{2}=1
$$

A transformada inversa pode ser facilmente definida. Com isso em mãos, podemos calcular o número de partículas criadas (7.21):

$$
\left\langle N_{\mathbf{k}}^{(1)}\right\rangle_{\eta_{2}}=\left|\beta_{k}\left(\eta_{1}, \eta_{2}\right)\right|^{2}
$$

Assim, podemos dizer que partículas são criadas do estado de vácuo inicial. Esse mecanismo é o responsável pela origem das perturbações cosmológicas na inflação. Uma definição dessas transformações e desses cálculos será dada de maneira mais completa nos próximos capítulos.

Definido o mecanismo responsável pela criação das perturbações cosmológicas na inflação só falta definirmos as condições iniciais das variáveis canônicas. Devido à condição de normalização (4.110), essa tarefa se reduz à determinação do tempo inicial $\eta_{i}$ com vácuo $\left|0, \eta_{i}\right\rangle$, que é o estado de menor energia. Podemos ver isso mais claramente da seguinte maneira. Como comentamos anteriormente, devido à inflação as perturbações que têm comprimentos de onda grande estavam dentro do horizonte de Hubble no começo da inflação e oscilavam muito rapidamente, podendo ser representadas por ondas planas em um espaço-tempo de Minkowski. Dessa forma, somente podemos quantizar as perturbações se existir um estado de vácuo (de Minkowski) no passado.

Queremos definir as condições iniciais de $Q_{\mathbf{k}, l}(\eta)$ e $Q_{\mathbf{k}, l}^{\prime}(\eta)$ em um tempo inicial $\eta_{i}$. Como essas flutuações se comportam como ondas planas, podemos fazer a substituição [31]:

$$
Q_{\mathbf{k}, l}=r_{\mathbf{k}, l} \mathrm{e}^{i \gamma_{\mathbf{k}, l}},
$$

onde $r_{\mathbf{k}, l}$ e a fase $\gamma_{\mathbf{k}, l}$ são números reais. Essas variáveis obedecem às equações de movimento para os modos das variáveis canônicas (4.111), que é a equação de um oscilador harmônico paramétrico com energia:

$$
\begin{aligned}
E_{\mathbf{k}, l} & =\frac{1}{2}\left(\left|Q_{\mathbf{k}, l}^{\prime}\right|^{2}+\omega_{\mathbf{k}, l}^{2}\left|Q_{\mathbf{k}, l}\right|^{2}\right) \\
& =\frac{1}{2}\left[\left(r_{\mathbf{k}, l}^{\prime}\right)^{2}+\frac{1}{r_{\mathbf{k}, l}^{2}}+\omega_{\mathbf{k}, l}^{2} r_{\mathbf{k}, l}^{2}\right],
\end{aligned}
$$

em que utilizamos que $r_{\mathbf{k}, l}^{2} \gamma_{\mathbf{k}, l}^{\prime}=1$, obtido da condição de normalização (4.110).

Queremos considerar agora as flutuações quânticas mínimas permitidas pelo princípio de incerteza de Heisenberg, ou seja, queremos considerar um estado de vácuo com energia mínima, em que as flutuações são geradas pelo princípio da incerteza. Logo, sendo a 
energia mínima do oscilador harmônico $E_{\mathbf{k}, l}\left(\eta_{i}\right)=\omega_{\mathbf{k}, l}$, temos que:

$$
r_{\mathbf{k}, l}\left(\eta_{i}\right)=\omega_{\mathbf{k}, l}^{-1 / 2}, \quad r_{\mathbf{k}, l}^{\prime}\left(\eta_{i}\right)=0
$$

Dessa forma, temos que as condições iniciais são:

$$
\begin{aligned}
& Q_{\mathbf{k}, l}\left(\eta_{i}\right)=\frac{1}{\sqrt{\omega_{\mathbf{k}, l}}} \mathrm{e}^{i \gamma_{\mathbf{k}, l}\left(\eta_{i}\right)}, \\
& Q_{\mathbf{k}, l}^{\prime}\left(\eta_{i}\right)=i \sqrt{\omega_{\mathbf{k}, l}} \mathrm{e}^{i \gamma_{\mathbf{k}, l}\left(\eta_{i}\right)},
\end{aligned}
$$

em que a fase $\gamma_{\mathbf{k}, l}$ é indeterminada. Esse estado de vácuo inicial é o vácuo de BunchDavies. Para a utilização que queremos nesse capítulo, somente a amplitude inicial é suficiente. Contudo, no Capítulo 7, faremos uma análise desse tópico mais detalhadamente e descreveremos o vácuo de Bunch-Davies.

Sumarizando, temos que as perturbações iniciais que evoluíram para formar as estruturas em larga escala foram formadas durante a inflação por flutuações quânticas em escalas nas quais as flutuações mínimas, dadas pelo princípio da incerteza de Heisenberg, são definidas sem ambigüidade. A inflação ainda é responsável por esticar essas perturbações iniciais até grandes escalas. Assim, a teoria da inflação resolve o problema da formação das estruturas, formando e esticando as perturbações primordiais.

\subsubsection{Espectro de Potências}

Como vimos acima, a teoria da inflação fornece o mecanismo para a formação e evolução das perturbações primordiais. Contudo, temos que saber se as previsões dessa teoria estão de acordo com as observações que fazemos hoje da RCF, que nos fornecem um espectro invariante de escala [24]. Dessa forma, vamos calcular o espectro de potências das perturbações.

Primeiramente, vamos calcular a função de correlação de dois pontos para as variáveis canônicas. Essa também é chamada de função de Green e é denotada por:

$$
\left\langle 0\left|\hat{Q}_{l}(\eta, \mathbf{x}), \hat{Q}_{l}\left(\eta^{\prime}, \mathbf{y}\right)\right| 0\right\rangle=G_{l}(x, y) .
$$

Como queremos o espectro de potências, vamos escrever a função de Green no espaço de Fourier:

$$
\left\langle 0\left|\hat{Q}_{l}(\eta, \mathbf{x}), \hat{Q}_{l}\left(\eta^{\prime}, \mathbf{y}\right)\right| 0\right\rangle=\int \frac{d^{3} k}{(2 \pi)^{3}} \underbrace{Q_{\mathbf{k}, l}(\eta) Q_{\mathbf{k}, l}^{*}\left(\eta^{\prime}\right)}_{G_{\mathbf{k}, l}\left(\eta, \eta^{\prime}\right)} e^{-i \mathbf{k}(\mathbf{x}-\mathbf{y})}
$$


O espectro de potências, então, é definido a partir da função de Green no espaço de Fourier para tempos iguais, $G_{\mathbf{k}, l}(\eta, \eta)$, e, correspondendo ao tempo em que os modos cruzam o horizonte de Hubble, $\eta=\eta_{h c}$, definido por $k=\sqrt{Z_{l}^{\prime \prime} / Z_{l}}$ :

$$
\mathcal{P}_{Q_{l}}(k)=\frac{1}{2 \pi^{2}} k^{3} \lim _{\eta \rightarrow \eta_{h c}} G_{\mathbf{k}}(\eta, \eta)=\left.\frac{1}{2 \pi^{2}} k^{3}\left|Q_{\mathbf{k}, l}(\eta)\right|^{2}\right|_{\eta=\eta_{h c}} .
$$

Como nosso interesse geral é a observação do espectro de potências, para podermos comprovar os resultados da teoria inflacionária, parametrizamos o espectro de potências da forma:

$$
\mathcal{P}_{Q_{l}} \propto k^{n_{s}-1},
$$

em que $n_{s}$ é o indice espectral, definido como sendo:

$$
n_{s}-1 \equiv \frac{d \ln \mathcal{P}_{Q}}{d \ln k} .
$$

Experimentalmente, o que é medido é o índice espectral.

Determinada a forma do espectro de potências e de sua análise, vamos agora substituir na variável $Q_{\mathbf{k}, l}$ cada modo de perturbação.

\subsubsection{Espectro das perturbações da densidade de um campo escalar}

Vamos primeiramente calcular o espectro de potências para as perturbações escalares, ou seja, para a variável de Mukhanov-Sasaki $v_{\mathbf{k}}(\eta)$. Como vimos de (4.89) [48]:

$$
v=z \mathcal{R}
$$

e como vamos calcular esse espectro no limite em que as perturbações são maiores que o horizonte de Hubble, $k \gg a H$, é mais interessante calcular o espectro de potências em função da perturbação da curvatura $\mathcal{R}$, que é uma quantidade conservada nesse limite. Assim, o espectro é definido como:

$$
P_{\mathcal{R}}=\frac{k^{3}}{2 \pi^{2}}\left|\frac{v_{\mathbf{k}}(\eta)}{z}\right|^{2} .
$$

Da conservação de $\mathcal{R}$ no limite $k \gg a H$, vemos que, por hipótese, as perturbações que reentram o horizonte de partículas hoje são as mesmas que cruzaram o raio de Hubble durante a inflação no instante $\eta=\eta_{h c}$, definido por $k_{h c}=\sqrt{z^{\prime \prime} / z} \sim H$. Assim, para calcularmos o espectro de potências nesse limite de tempos grandes, vamos relacionar o espectro de potências para escalas maiores que o raio de Hubble com o espectro de potências no instante em que os modos cruzam o horizonte, $\eta_{h c}(k)$ ou $t_{h c}(k)$, em tempo próprio. Para isso iremos utilizar que $v_{\mathbf{k}} \sim z$, para escalas maiores que o horizonte de Hubble $k \ll\left(z^{\prime \prime} / z\right)^{1 / 2}(\sim H)$ e vemos que : 


$$
\mathcal{P}_{\mathcal{R}}=\frac{k^{3}}{2 \pi^{2}} z^{-2}(t)\left[\frac{z(t)}{z\left(t_{h c}(k)\right)}\right]^{2}\left|v_{\mathbf{k}}\left(t_{h c}(k)\right)\right|^{2} .
$$

Se considerarmos que a equação de movimento não muda durante o tempo, ou seja, para o tempo do cruzamento do horizonte $\mathcal{H}$ e $\varphi^{\prime}$ são proporcionais, $z\left(t_{h c}(k)\right) \sim a\left(t_{h c}(k)\right)$ e $v_{\mathbf{k}}\left(t_{h c}(k)\right)$ é constante em escalas menores que o horizonte de Hubble, podemos ver que:

$$
\mathcal{P}_{\mathcal{R}} \sim \frac{k^{3}}{2 \pi^{2}} a^{-2}\left(t_{h c}(k)\right)\left|v_{\mathbf{k}}\left(t_{i}\right)\right|^{2}
$$

Uma vez que as condições iniciais são dadas por (4.124), ou seja, são as condições iniciais do vácuo de Bunch-Davies adiabático, temos que $\left|v_{\mathbf{k}}\left(t_{i}\right)\right|=\left|\omega_{\mathbf{k}}\right|^{-1} \sim k^{2}$, fora do horizonte, então o espectro de potências tem a forma:

$$
\mathcal{P}_{\mathcal{R}} \sim k^{3} \frac{H^{2}}{k^{2}} \frac{1}{k}=H^{2},
$$

em que utilizamos $a^{-1}\left(t_{h c}(k)\right)=H / k$. Assim, temos que o espectro de potências das perturbações é invariante de escala e depende de $H^{2}$. Sabemos de (3.33) que o parâmetro de Hubble é quase constante na inflação com rolagem lenta, mas exibe uma dependência $\operatorname{com} \eta$.

Devido à leve dependência de $H^{2}$ com o tempo conforme, teremos que $n_{s}$ não será exatamente um, mas sim [63]:

$$
n_{s}=1-2 \eta_{s r}-6 \epsilon=1-2 \delta-4 \epsilon,
$$

que durante a inflação com condições de rolagem lenta em que $\epsilon,\left|\eta_{s r}\right|, \delta \ll 1$, será sempre $n_{s} \lesssim 1$. Isso nos mostra que o índice espectral medido sempre será menor que um, ou seja, as perturbações escalares tenderão sempre levemente para o vermelho do espectro.

\subsubsection{Espectro de potências das ondas gravitacionais}

De maneira análoga, podemos calcular o espectro de potências das ondas gravitacionais. De (4.90), podemos escrever o espectro da forma:

$$
\mathcal{P}_{\text {og }}=\frac{k^{3}}{2 \pi^{2}} \frac{4}{M_{p}^{2}} \sum_{\lambda=+, \times}\left|\frac{\mu_{\lambda}(\eta)}{a}\right|^{2} .
$$

Não vamos calcular aqui esse espectro, pois foge do escopo da dissertação. Para uma análise mais detalhada do espectro das ondas gravitacionais e suas propriedades, ver referência [63]. 


\subsubsection{Relação de consistência}

Por fim, vamos introduzir um parâmetro que relaciona os espectros das ondas gravitacionais e das perturbações escalares. A relação de consistência é uma razão definida como [63]:

$$
r \equiv \frac{1}{2} \frac{\mathcal{P}_{\text {og }}}{\mathcal{P}_{\mathcal{R}}}=8 \epsilon
$$

Essa razão entre quantidades puramente observacionais representa um teste direto de modelos inflacionários de um campo escalar com rolagem lenta. Isso pode ser visto, uma vez que, para qualquer modelo inflacionário de lei de potência, $V(\varphi) \propto \varphi^{\alpha}, \alpha>0$, temos que:

$$
n_{s}=1-\frac{\alpha+2}{N}, r=\frac{4 \alpha}{N}
$$

em que $N$ é o número de $e$-folds. Para o modelo de inflação caótica que estamos estudando, com $\alpha=2$, e um período inflacionário que dura aproximadamente 70 e-folds, que condiz com nossas previsões, teríamos $n_{s} \sim 0,94$ e $r \sim 0,11$.

Podemos comparar então com as observações do WMAP [24] que nos fornecem:

$$
\begin{array}{r}
n_{s}=0,94_{-0,013}^{+0,014}, 68 \% C L, \\
r<0.2,95 \% C L .
\end{array}
$$

Esses valores são compatíveis com os obtidos pelo modelo inflacionário caótica com potencial quadrático ${ }^{3}, V(\varphi)=m \varphi^{2} / 2$, o que nos diz que esse é um modelo robusto para a inflação.

\footnotetext{
${ }^{3}$ Não é válido para o potencial $\operatorname{com} \alpha=4$.
} 


\section{Capítulo 5}

\section{Efeito Schwinger Estático}

Efeitos de criação de pares de elétrons e pósitrons devido à perturbação do vácuo por um campo elétrico externo da Eletrodinâmica Quântica (QED) são fenômenos não-perturbativos não triviais que vêm sendo estudados há mais de cinco décadas. Desde os trabalhos seminais de Sauter [69], Euler e Heisenberg [70] e Weisskopf [71], foram descobertos os fenômenos de polarização do vácuo quântico da QED, assim como espalhamentos fótonfóton e produção de pares a partir do vácuo. Contudo, foi somente com Julian Schwinger, em 1951 [72], que o efeito de produção de partículas do vácuo instável da QED por um campo elétrico estático foi estudado. Dessa forma, foi dado a esse efeito o nome de efeito Schwinger estático.

O estudo desse fenômeno é de grande importância para a QED, uma vez que revela nuances sobre a estrutura instável do vácuo quântico, assim como para o estudo da QED em um limite não-perturbativo. Muitos efeitos quânticos surgem do fato do vácuo apresentar essas flutuações, pois isso modifica a idéia clássica de seu comportamento e nos faz vê-lo como um meio polarizável. A QED é uma teoria que apresenta-se muito bem comprovada, sendo que experimentos como o Lamb shift [89, 90] (que consiste na diferença de energia entre os níveis $2 \mathrm{~S}$ e $2 \mathrm{P}$ do hidrogênio), do momento magnético anômalo do elétron [91, 92, 93], do espalhamento Delbrück [94], espalhamento Compton não-linear [95], efeito Casimir [96, 97, 98], entre outros, foram observados experimentalmente e comprovam as previsões da QED com incrível precisão. Contudo, todos esses efeitos são efeitos perturbativos da QED. Efeitos não-perturbativos da QED como a produção no espalhamento fóton-fóton e o efeito Schwinger são muito fracos e ainda não foram detectados experimentalmente. Sua comprovação é de grande importância para a QED.

Outra dificuldade que o efeito Schwinger enfrentou foi a de seu estudo teórico, uma vez que esse efeito é altamente não-linear e não-perturbativo. Schwinger, em seu trabalho clássico de 1951, analisou o caso de um campo fermiônico acoplado minimamente a um campo externo eletromagnético. Ele o fez utilizando o método da Lagrangeana efetiva. A existência de uma parte imaginária nessa Lagrangeana efetiva, quando $\mathbf{E}^{2}-\mathbf{B}^{2}>0$, com $\mathbf{E}$ e $\mathbf{B}$ constantes, foi atribuída, por Schwinger, à instabilidade do vácuo e representava a 
probabilidade de criação de pares por unidade de volume. Ele calculou essa probabilidade exatamente para o caso do campo elétrico estático, que é o que chamamos aqui de efeito Schwinger estático. Nesse trabalho Schwinger logo verificou que eram necessários campos muito fortes, praticamente impossíveis de se reproduzirem em laboratório, para que esse efeito pudesse ser observado.

O efeito Schwinger ainda não foi detectado experimentalmente. Para que isso seja possível, são necessários campos elétricos estáticos muito fortes com energia da ordem de $E_{\text {crit }} \sim 1.3 \times 10^{18} \mathrm{~V} / \mathrm{m}$, como mostraremos adiante. Campos desse tipo ainda não são possíveis de serem realizados experimentalmente. Contudo, experimentos como o ELI (Extreme Light Infrastructure) estão próximos de chegar a essa energia [106] e da comprovação do efeito Schwinger e da QED para limites não-perturbativos.

O interesse pelo efeito Schwinger foi, por muito tempo, pequeno, uma vez que era tido que dificilmente seria observado. Contudo, nos anos 70, já após a validação da QED por seus testes de precisão e do grande desenvolvimento teórico da teoria quântica de campos (TQC), o Efeito Schwinger voltou a ser de interesse para a comunidade científica. Isso ocorreu, em parte, devido ao avanço das técnicas experimentais apontando para uma possível observação do fenômeno ou de alguma generalização dele, assim como devido às analogias com outros mecanismos de criação de partículas. Isso mostra a importância do efeito Schwinger e de sua verificação experimental, que corresponderia à validação de um efeito da QED não-perturbativa.

Assim, surgiram diferentes descrições do efeito Schwinger baseadas em diferentes descrições de processos na TQC - além daquelas provenientes de analogias. Baseado no trabalho pioneiro de L. V. Keldysh [73] sobre ionização, E. Brezin e C. Itzykson, em 1970, calcularam o Efeito Schwinger estático fazendo uma generalização do formalismo utilizado por Schwinger, para o cálculo do campo eletromagnético interagindo com uma corrente externa. Utilizando o formalismo da matriz S, ou matriz de interação, calcularam para o campo fermiônico e para o campo escalar ou bosônico carregado(campo dos bóson com partículas de spin inteiro) a probabilidade por unidade de volume e de tempo de não emitir pares e, assim, retomaram os resultados obtidos por Schwinger. Nessa trabalho, também há uma generalização do efeito Schwinger estático para o caso dinâmico, que iremos estudar no próximo capítulo. Esse método de cálculo é que fornece explicitamente os limites experimentais para a observação do efeito e seu formalismo é mais adequado para a descrição de experimentos, logo chamamos de descrição via teoria de espalhamento.

Com o passar dos anos, muito outros métodos foram desenvolvidos para se tratar esse importante fenômeno, tais como tunelamento [99, 100], detectores de partículas [101, 102], método do funcional de onda [103, 104], entre outros. Podemos ver em [101] que esses métodos nem sempre nos levam ao mesmo resultado. Na referência [105] é inclusive discutida a validade de alguns desses métodos e suas equivalências.

O efeito Schwinger tem recebido atenção da comunidade científica, pois oferece um 
entendimento para uma grande gama de efeitos não-perturbativos. Um desses efeitos é a criação de partículas por campos gravitacionais externos. Para essa analogia, é interessante analisar também o caso do campo escalar complexo interagindo com um campo elétrico externo, além de também representar uma simplificação do campo de Dirac. Devido a essa analogia, outros formalismos foram utilizados, ainda, para descrever o efeito Schwinger, assim como o formalismo dos modos normais ou das transformadas de Bogoliubov.

Apresentaremos nesse capítulo a descrição do efeito Schwinger para campos escalares carregados e fermiônicos (campo de Dirac), nos casos que isso for possível, com o intuito de fazer uma revisão geral, extensa e detalhada, dos métodos existentes para o estudo desse fenômeno. Primeiramente, iremos descrever o problema, demostrando como tratamos a TQC para campos interagindo com potenciais externos. Fazemos esse cálculo para o campo escalar complexo e fermiônico. Apesar de necessário para o capítulo, a descrição do problema para um campo escalar é de grande importância para a dissertação, uma vez que será diretamente utilizado no capítulo central (Capítulo 7) em que fazemos a analogia com a inflação.

A criação de partículas foi determinada via teoria de espalhamento, transformadas de Bogoliubov e seu formalismo complementar dos estados squeezed. A descrição via teoria de espalhamento, baseada em [74, 75], apesar de completa e extensa, além de demostrar um dos tratamentos do efeito Schwinger, tem como principal importância a obtenção dos limites observacionais. Isso nos faz verificar a ordem de grandeza da precisão que experimentos ainda têm que alcançar para a detecção desse efeito. De importância principal para a dissertação é a descrição via transformadas de Bogoliubov, uma vez que esse será o método que utilizaremos para a analogia com a criação de partículas na inflação. A descrição via estados squeezed nos auxilia a ilustrar de maneira mais clara a criação de partículas.

Convém salientar que o campo será prescrito a priori, ou seja, efeitos de backreaction, não serão considerados.

No Apêndice A, calculamos o efeito Schwinger estático pelo método das Lagrangeanas efetivas, por sua motivação histórica.

\subsection{Formalismo geral e quantização canônica}

Nessa seção vamos mostrar rapidamente o formalismo geral de um campo quântico interagindo com um campo externo clássico, que no caso é um campo eletromagnético. Faremos isso primeiramente para o campo escalar complexo e em seguida para o campo fermiônico. 


\section{Campo escalar complexo}

Vamos tratar inicialmente do campo escalar complexo interagindo com um potencial eletromagnético clássico. Esse campo representa partículas com spin zero carregadas, uma vez que associa números complexos com todos os pontos do espaço tempo, o que nos permite distinguir entre partículas e antipartículas, que devem carregar números quânticos de carga oposta. Para isso, é necessária a introdução de um dubleto de campos hermitianos $\phi_{1}$ e $\phi_{2}$, representados pela quantidade complexa:

$$
\phi=\frac{\phi_{1}+i \phi_{2}}{\sqrt{2}}
$$

e seu hermitiano conjugado. Assim, o sistema do campo escalar complexo interagindo com o campo eletromagnético é descrito pela ação:

$$
\begin{aligned}
S & =\int_{R} d^{4} x \mathcal{L}=\int d^{4} x\left(\mathcal{L}\left(\phi_{1}\right)+\mathcal{L}\left(\phi_{2}\right)\right) \\
& =-\frac{1}{2} \int d^{4} x\left[D_{\mu} \phi\left(D^{\mu} \phi\right)^{*}-m^{2} \phi \phi^{*}\right],
\end{aligned}
$$

onde $\phi \neq \phi^{*}, D_{\mu}=\partial_{\mu}+i e A_{\mu}$, e é a carga do campo e $m$ é a massa da partícula escalar, em que é possível identificar-se a Lagrangeana de interação,

$$
\mathcal{L}_{\text {int }}=\frac{i e}{2}\left[\left(\partial_{\mu} \phi\right) A^{\mu} \phi^{*}-A_{\mu} \phi\left(\partial^{\mu} \phi^{*}\right)\right]+e^{2} A^{2} \phi \phi^{*} .
$$

A partir da Lagrangeana definida acima, podemos aplicar o princípio da ação integral, introduzido por Schwinger, que afirma que a ação deve ser estacionária para qualquer variação infinitesimal do campo $\delta \phi$, que se anule na fronteira da região $\mathrm{R}$ de integração acima. Assim, como $\delta S=0$, obtemos as equações de Euler-Lagrange ou equações de movimento para os campos (variando $\phi^{*}$ obtemos as equações de movimento para $\phi$ e vice-versa). Logo, para $\phi$ temos que:

$$
\left(D_{\mu} D^{\mu}+m^{2}\right) \phi=0
$$

e analogamente para $\phi^{*}$. Essa equação é covariate e também é chamada de equação de Klein-Gordon.

Para podermos estudar separadamente o comportamento de cada modo de Fourier, reescrevemos o sistema acima no espaço de momentos, decompondo o campo através de uma transformada de Fourier:

$$
\phi(t, \mathbf{x})=\frac{1}{(2 \pi)^{3}} \int d^{3} \mathbf{k} \phi_{k}(t) e^{i \mathbf{k} \cdot \mathbf{x}}
$$


Uma solução geral da equação de Klein-Gordon (5.5) pode ser escrita como uma decomposição:

$$
\phi(x)=\frac{1}{(2 \pi)^{3}} \int \frac{d^{3} \mathbf{k}}{\sqrt{2 \omega_{\mathbf{k}}}}\left(a_{\mathbf{k}} e^{-i \mathbf{k x}}+b_{\mathbf{k}}^{*} e^{i \mathbf{k x}}\right),
$$

em que $a_{\mathbf{k}}$ e $b_{\mathbf{k}}^{*}$ são os coeficientes da parte positiva e negativa da solução. Temos uma equação equivalente para $\phi^{*}$.

No espaço do momentos reescrevemos a ação (5.3), inserindo a decomposição (5.6):

$$
S=-\int d t \int_{\mathbb{R}^{3}} d^{3} \mathbf{k}\left\{-\dot{\phi}_{\mathbf{k}} \dot{\phi}_{\mathbf{k}}^{*}+\omega_{\mathbf{k}}^{2}(t) \phi_{\mathbf{k}} \phi_{\mathbf{k}}^{*}\right\}
$$

onde:

$$
\omega_{\mathbf{k}}^{2}(t)=k^{2}+m^{2}+2 i e k_{\mu} A^{\mu}+e^{2} A^{2} .
$$

Deduzindo as equações de movimento nessa decomposição, podemos ver que os modos $\mathbf{k}$ se desacoplam e na verdade representam uma coleção de osciladores harmônicos paramétricos:

$$
\ddot{\phi}_{\mathbf{k}}+\omega_{\mathbf{k}}^{2}(t) \phi_{\mathbf{k}}=0 \text {. }
$$

É interessante também formular o problema no formalismo Hamiltoniano, que nos fornece uma visão diferente do sistema, pois ao invés de recair em equações de segundo grau como o formalismo Lagrangeano, fornece equações de primeiro grau, as equações de Hamilton definidas no espaço de fase. Para isso precisamos definir o momento:

$$
\left\{\begin{array}{l}
p_{\mathbf{k}}=\frac{\partial \mathcal{L}_{\mathbf{k}}}{\partial \dot{\phi}_{\mathbf{k}}^{*}}=\dot{\phi}_{\mathbf{k}} \\
p_{\mathbf{k}}^{*}=\frac{\partial \mathcal{L}_{\mathbf{k}}}{\partial \dot{\phi}_{\mathbf{k}}^{*}}=\dot{\phi}_{\mathbf{k}}^{*}
\end{array},\right.
$$

e fazendo uma transformada de Legendre, obtemos a Hamiltoniana do sistema:

$$
\begin{aligned}
H & =\int_{\mathbb{R}^{3}} d^{3} \mathbf{k} H_{\mathbf{k}}=\int_{\mathbb{R}^{3}}\left(p_{\mathbf{k}} \dot{\phi}_{\mathbf{k}}^{*}+p_{\mathbf{k}}^{*} \dot{\phi}_{\mathbf{k}}-\mathcal{L}_{\mathbf{k}}\right) \\
& =\int_{\mathbb{R}^{3}}\left(p_{\mathbf{k}} p_{\mathbf{k}}^{*}+\omega_{\mathbf{k}}^{2}(t) \phi_{\mathbf{k}} \phi_{\mathbf{k}}^{*}\right)
\end{aligned}
$$

que também podemos reconhecer como uma coleção de Hamiltonianas de um oscilador harmônico com freqüência $\omega_{\mathbf{k}}$. 


\section{Quantização Canônica}

Passaremos agora para o procedimento de quantização canônica. Como vimos no capítulo anterior, para quantizarmos o campo, as variáveis canônicas são promovidas a operadores:

$$
\left\{\begin{array}{l}
\phi \rightarrow \hat{\phi} \Longrightarrow \phi_{\mathbf{k}} \rightarrow \hat{\phi}_{\mathbf{k}} \\
p \rightarrow \hat{p}=\frac{\partial \mathcal{L}}{\partial \dot{\phi}^{*}}=\dot{\phi} \Longrightarrow p_{\mathbf{k}} \rightarrow \hat{p}_{\mathbf{k}}=\dot{\phi}_{\mathbf{k}}
\end{array}\right.
$$

que devem obedecer às relações de comutação:

$$
\left\{\begin{array}{ll}
{[\phi(\mathbf{x}, t), p(\mathbf{y}, t)]=i \delta(\mathbf{x}-\mathbf{y}),} & {[\phi(\mathbf{x}, t), \phi(\mathbf{y}, t)]=[p(\mathbf{x}, t), p(\mathbf{y}, t)]=0} \\
{\left[\phi_{\mathbf{k}}(t), p_{\mathbf{k}^{\prime}}(t)\right]=\delta\left(\mathbf{k}-\mathbf{k}^{\prime}\right),} & {\left[\phi_{\mathbf{k}}(t), \phi_{\mathbf{k}^{\prime}}(t)\right]=\left[p_{\mathbf{k}}(t), p_{\mathbf{k}^{\prime}}(t)\right]=0}
\end{array} .\right.
$$

Com esses operadores em mãos, podemos escrever o operador Hamiltoniano do campo escalar da forma:

$$
\hat{H}_{\mathbf{k}}=\hat{p}_{\mathbf{k}} \hat{p}_{\mathbf{k}}^{\dagger}+\omega_{\mathbf{k}}^{2}(t) \hat{\phi}_{\mathbf{k}} \hat{\phi}_{\mathbf{k}}^{\dagger}
$$

Com a quantização canônica, como o campo agora é um operador, os coeficientes $a_{\mathbf{k}}$ e $b_{\mathbf{k}}$ também tornam-se operadores, $\hat{a}_{\mathbf{k}}$ e $\hat{b}_{\mathbf{k}}$. Essa é a segunda quantização, em que a variável campo se torna um operador que age nos estados quânticos de partículas. Suas regras de comutação são definidas, para o campo escalar complexo, em tempos iguais, como:

$$
\begin{cases}{\left[\hat{a}_{\mathbf{k}}, \hat{a}_{\mathbf{k}}^{\dagger}\right]=\delta\left(\mathbf{k}-\mathbf{k}^{\prime}\right), \quad\left[\hat{a}_{\mathbf{k}}, \hat{a}_{\mathbf{k}^{\prime}}\right]=\left[\hat{a}_{\mathbf{k}}^{\dagger}, \hat{a}_{\mathbf{k}^{\prime}}^{\dagger}\right]=0} \\ {\left[\hat{b}_{\mathbf{k}}, \hat{b}_{\mathbf{k}^{\prime}}^{\dagger}\right]=\delta\left(\mathbf{k}-\mathbf{k}^{\prime}\right), \quad\left[\hat{b}_{\mathbf{k}}, \hat{b}_{\mathbf{k}^{\prime}}\right]=\left[\hat{b}_{\mathbf{k}}^{\dagger}, \hat{b}_{\mathbf{k}^{\prime}}^{\dagger}\right]=0}\end{cases}
$$

Logo, podemos reescrever a Hamiltoniana em função desses operadores:

$$
\hat{H}_{\mathbf{k}}=\omega_{\mathbf{k}}(t)\left(\hat{a}_{\mathbf{k}}^{\dagger} \hat{a}_{\mathbf{k}}+\hat{b}_{\mathbf{k}}^{\dagger} \hat{b}_{\mathbf{k}}\right)
$$

Sabe-se que a Hamiltoniana tem auto-estados com autovalores dados pela energia. Como vimos no capítulo anterior, da positividade da Hamiltoniana quadrática sabemos que deve existir um estado com energia mais baixa, $|0\rangle$, tal que $H|0\rangle=E_{0}|0\rangle$. Logo, podemos definir o vácuo, como sendo:

$$
\hat{a}_{\mathbf{k}}|0\rangle=\hat{b}_{\mathbf{k}}|0\rangle=0
$$

ou seja, o estado que não contém partículas ou antipartículas. Assim, os operadores $\hat{a}_{\mathbf{k}}$ e $\hat{b}_{\mathbf{k}}$ são chamados de operadores de aniquilação de partículas e anti-partículas, respectivamente, e $\hat{a}_{\mathbf{k}}^{\dagger} \mathrm{e} \hat{b}_{\mathbf{k}}^{\dagger}$ de operadores de criação de partículas e anti-partículas, respectivamente. 
Na descrição de Heisenberg, na qual os operadores é que carregam a evolução temporal, os estados quânticos geram um espaço de Hilbert onde esses operadores agem, o espaço de Fock. Os operadores de criação criam um estado que contém partículas e antipartículas, respectivamente:

$$
\hat{a}_{\mathbf{k}}^{\dagger}|0\rangle=|1 \mathbf{k}\rangle, \quad \hat{b}_{\mathbf{k}}^{\dagger}|0\rangle=|1 \overline{\mathbf{k}}\rangle .
$$

O número de partículas e antipartículas é dado por $\hat{N}_{\mathbf{k}}=\hat{a}_{\mathbf{k}}^{\dagger} \hat{a}_{\mathbf{k}}$ e $\hat{\bar{N}}_{\mathbf{k}}=\hat{b}_{\mathbf{k}}^{\dagger} \hat{b}_{\mathbf{k}}$, seu valor esperado no vácuo e em um estado de muitas partículas é dado por:

$$
\left\langle 0\left|\hat{N}_{\mathbf{k}}\right| 0\right\rangle=0, \quad\left\langle n\left|\hat{N}_{\mathbf{k}}\right| n\right\rangle=n_{\mathbf{k}}, \forall \mathbf{k} .
$$

Apesar do procedimento de quantização ser muito semelhante ao dos campos livres, no caso em que há um campo externo a invariância translacional, no espaço e no tempo, é quebrada, e assim não podemos definir uma base ortonormal única. Ou seja, o estado de vácuo definido em (5.18) é agora dependente da base em que a quantização foi feita.

\section{Campo fermiônico}

Feita a descrição do campo escalar complexo, podemos partir para o campo fermiônico ou campo de Dirac, no caso aqui associado aos férmions de spin $1 / 2$. O procedimento é basicamente análogo, somente sendo necessário tomar cuidado com as peculiaridades do campo fermiônico.

A equação de Dirac para o campo fermiônico $\psi(x)$ é dada por:

$$
\left(i \gamma^{\mu} D_{\mu}-m\right) \psi(x)=0
$$

em que $\gamma_{\mu}$ são as matrizes de Dirac, e é a carga do elétron e $m$ é a massa do elétron.

A Lagrangeana que fornece essa equação de Dirac é dada por:

$$
\begin{aligned}
\mathcal{L} & =\bar{\psi}(x)\left(i \gamma_{\mu} D^{\mu}-m\right) \psi(x) \\
& =i \bar{\psi} \gamma^{\mu} \partial_{\mu} \psi-\psi \bar{\psi} \underbrace{-e \bar{\psi} \gamma_{\mu} A^{\mu} \psi}_{\mathcal{L}_{\text {int }}},
\end{aligned}
$$

onde $\bar{\psi} \equiv \psi^{\dagger} \gamma_{0}$ é o adjunto do campo. Uma vez que $\psi$ é complexo, podemos variar $\psi$ e $\bar{\psi}$ independentemente para obtermos as equações de movimento (que são as equações de Dirac). O adjunto também tem uma equação de Dirac chamada de equação de Dirac adjunta. 
Substituindo a derivada covariante na Lagrangeana do sistema, derivamos as equações de movimento em relação a $\bar{\psi}$ e $\psi$, respectivamente:

$$
\left\{\begin{array}{l}
i \gamma^{\mu} \partial_{\mu} \psi-m \psi=e \psi \gamma_{\mu} A^{\mu} \\
i \partial_{\mu} \bar{\psi} \gamma^{\mu}+m \bar{\psi}=-e \bar{\psi} \gamma_{\mu} A^{\mu}
\end{array}\right.
$$

em que o lado esquerdo corresponde à equação de Dirac livre (ou de Dirac adjunta), enquanto no lado direito temos uma termo de corrente, que corresponde à interação com o campo eletromagnético.

A equação de Dirac exige a presença de antipartículas. Podemos ver isso decompondo o campo de Dirac, da mesma forma feita com o campo escalar complexo, em termos das soluções de onda plana da equação de Dirac. A decomposição é descrita na forma (ou a solução geral arbitrária da equação de Dirac):

$$
\psi(x)=\frac{1}{(2 \pi)^{3}} \int \frac{d^{3} k}{\omega_{k}(t) / m} \sum_{r=1,2}[d_{r}(k) \underbrace{u^{(r)}(k) \mathrm{e}^{-i k \cdot x}}_{\psi^{(+)}}+f_{r}^{\dagger}(k) \underbrace{v^{(r)}(k) \mathrm{e}^{i k \cdot x}}_{\psi^{(-)}}]
$$

em que $d_{r}$ e $f_{r}$ são coeficientes dessa expansão e $\psi^{(+)}$e $\psi^{(-)}$são, respectivamente, as soluções de onda plana de energia positiva e negativa, com a condição de que $k_{0}$ seja positivo. Para o campo adjunto é só tomar o complexo conjugado de (5.24) e multiplicar por $\gamma_{0}$. Podemos ver que essa expansão contém um número quântico a mais, denominado por $r$, ou índice espinorial. A origem desse número quântico pode ser vista se substituirmos a expansão acima na equação de Dirac, o que implica que temos duas equações:

$$
\left(\gamma^{\mu} k_{\mu}-m\right) u(k)=0, \quad\left(\gamma^{\mu} k_{\mu}+m\right) v(k)=0 .
$$

Dessa forma, as soluções são compostas por matrizes coluna com quatro componentes, pois as matrizes de Dirac são $4 \times 4$. Essa matrizes têm uma estrutura de bloco $2 \times 2$ e logo, as soluções de (5.25) tem uma estrutura $2 \times 2$. Essas soluções são chamadas espinores e com isso o campo fermiônico também recebe o nome de campo espinorial.

Para escrevermos o campo fermiônico no formalismo Hamiltoniano, definimos o momento canonicamente conjugado:

$$
\hat{\Pi}_{\psi}=\frac{\partial \mathcal{L}}{\partial\left(\partial_{0} \psi\right)}=i \bar{\psi} \gamma^{0}=i \psi^{\dagger}, \quad \hat{\Pi}_{\bar{\psi}}=\frac{\partial \mathcal{L}}{\partial\left(\partial_{0} \bar{\psi}\right)}=i \gamma^{0} \psi
$$

Com isso, a hamiltoniana do sistema é dada, depois de uma transformação de Legen- 
dre, por:

$$
\begin{aligned}
H & =\int d^{3} x \frac{i}{2}\left[\left(\bar{\psi} \gamma^{0} D^{0} \psi-D^{0} \bar{\psi} \gamma^{0} \psi\right)-\eta^{\mu \nu} \mathcal{L}\right] \\
& =\int d^{3} x \frac{1}{2}\left(\hat{\Pi}_{\psi} D^{0} \psi-\left(D^{0} \bar{\psi}\right) \hat{\Pi}_{\bar{\psi}}\right)
\end{aligned}
$$

ou em termos da expansão (5.24):

$$
H_{k}=\sum_{r} \omega_{k}(t)\left[d_{r}(k) d_{r}(k)+f_{r}(k) f_{r}^{\dagger}(k)\right]
$$

em que $\omega_{\mathbf{k}}(t)=\sqrt{(\mathbf{p}-e \mathbf{A})^{2}+m^{2}}$

Observamos que a Hamiltoniana acima não é positiva definida, diferentemente do caso escalar. Logo, a energia não é limitada inferiormente. Para resolvermos esse problema impõe-se que:

$$
\left\{f(k), f^{\dagger}(k)\right\}=\text { número } \mathrm{c},
$$

e com isso, a menos de uma constante,

$$
H_{k}=\sum_{r} \omega_{k}(t)\left[d_{r}^{\dagger}(k) d_{r}(k)+f_{r}^{\dagger}(k) f_{r}(k)\right]
$$

\section{Quantização canônica}

Para o campo fermiônico, introduzindo os operadores de campo e momento da mesma forma que o campo escalar, vemos que, para mantermos a positividade da energia, temos que utilizar anticomutadores na quantização. Logo, os operadores de campo e momento devem obedecer a:

$$
\begin{aligned}
\left\{\hat{\psi}_{\alpha}(\mathbf{x}, t), \hat{\Pi}_{\psi \beta}(\mathbf{y}, t)\right\} & =\delta_{\alpha \beta} \delta(\mathbf{x}-\mathbf{y}) \\
\left\{\hat{\psi}_{\alpha}(\mathbf{x}, t), \hat{\psi}_{\beta}(\mathbf{y}, t)\right\} & =\left\{\hat{\Pi}_{\alpha \psi}(\mathbf{x}, t), \hat{\Pi}_{\beta \psi}(\mathbf{y}, t)\right\}=0
\end{aligned}
$$

Assim, vemos a principal diferença entre o processo de quantização canônica do campo escalar e do campo fermiônico.

Com isso, o Hamiltoniano do campo fermiônico é escrito da forma:

$$
\hat{H}=\frac{1}{2}\left[\hat{\bar{\psi}} D^{0} \hat{\Pi}_{\psi}-\left(D^{0} \hat{\Pi}_{\bar{\psi}}\right) \psi\right]
$$

Fazendo o procedimento de segunda quantização de maneira análoga para o campo fermiônico, mas seguindo as regras de anticomutação, temos que: 


$$
\left\{\begin{array}{ll}
\left\{\hat{d}_{\mathbf{k}}^{(r)}, \hat{d}_{\mathbf{k}}^{(s) \dagger}\right\}=\delta_{r s} \delta\left(\mathbf{k}-\mathbf{k}^{\prime}\right), & \left\{\hat{d}_{\mathbf{k}}^{(r)}, \hat{d}_{\mathbf{k}^{\prime}}^{(s)}\right\}=\left\{\hat{d}_{\mathbf{k}}^{(r) \dagger}, \hat{d}_{\mathbf{k}^{\prime}}^{(s) \dagger}\right\}=0 \\
\left\{\hat{f}_{\mathbf{k}}^{(r)}, \hat{f}_{\mathbf{k}^{\prime}}^{(s) \dagger}\right\}=\delta_{r s} \delta\left(\mathbf{k}-\mathbf{k}^{\prime}\right), & \left\{\hat{f}_{\mathbf{k}}^{(r)}, \hat{f}_{\mathbf{k}^{\prime}}^{(s)}\right\}=\left\{\hat{f}_{\mathbf{k}}^{(r) \dagger}, \hat{f}_{\mathbf{k}^{\prime}}^{(s) \dagger}\right\}=0
\end{array} .\right.
$$

Logo, em termos desses operadores, podemos reescrever o operador Hamiltoniano:

$$
\hat{H}_{k}=\sum_{r} \omega_{\mathbf{k}}(t)\left[\hat{d}_{\mathbf{k}}^{(r) \dagger} \hat{d}_{\mathbf{k}}^{(r)}+\hat{f}_{\mathbf{k}}^{(r) \dagger} \hat{f}_{\mathbf{k}}^{(r)}\right]
$$

Podemos, para o campo fermiônico, definir o espaço de Hilbert de maneira análoga à feita para o campo escalar complexo, porém o estado criado terá um número quântico a mais, o spin, definido pelo índice $r$.

\subsection{Evolução Temporal}

Nesta seção iremos estudar a dinâmica dos efeitos do vácuo quântico induzidos por um campo externo, no caso o efeito Schwinger estático. Com o formalismo estabelecido na seção anterior é possível agora estudar a criação de partículas induzida pelo campo externo, ou seja a interação entre os campos escalar complexo ou o campo fermiônico com um campo eletromagnético externo.

Como mencionamos a dinâmica do efeito Schwinger pode ser descrita por diversas técnicas baseadas ou em diferentes descrições da TQC, ou em diferentes analogias feitas para o efeito e que levam a diferentes interpretações do fenômeno. Apresentaremos aqui a descrição do efeito Schwinger em três diferentes métodos em que a criação de partículas surge do formalismo de maneiras distintas. Cada uma das descrições do efeito Schwinger apresentada tem um intuito diferente. Primeiramente, passaremos por dois métodos baseados na matriz S: via teoria de espalhamento, muito utilizada em física de partículas, que nos apresenta uma boa descrição do fenômeno para aplicação experimental; e via transformadas de Bogoliubov, muito utilizada em problemas em presença de campos gravitacionais, assim como em óptica quântica, será apresentada com o intuito de fazer analogia com o problema de criação de partículas na presença de campos gravitacionais, no Capítulo 7. Apesar de ambos basearem-se na descrição da interação através da matriz S, apresentam diferenças não só em sua formulação como na interpretação do resultado, ou seja, da criação de partículas. Por fim, utilizaremos o formalismo dos estados squeezed, comumente utilizado na mecânica quântica, mas que também aparece em analogias para problemas com campos gravitacionais, e que utilizamos para ilustrarmos a criação de partículas. Essas técnicas foram escolhidas, também, por serem métodos mais amplamente utilizados na literatura e, portanto mais familiares. 


\subsubsection{Dinâmica via teoria de espalhamento}

Estudaremos nesta Seção a criação de partículas via teoria de espalhamento. O tratamento do efeito Schwinger via teoria de espalhamento foi feito pela primeira vez por C. Itzykson e E. Brezin em 1970 [74]. É uma analogia ao cálculo feito por Schwinger, utilizando uma interpretação da interação entre o campo de matéria e o campo eletromagnético como um problema de espalhamento. Esse tratamento se propõe a calcular a probabilidade de não emitir nenhuma partícula por unidade de volume e tempo, em que a criação de partículas é interpretada como essa probabilidade ser diferente da unidade. Essa interpretação do efeito Schwinger como um espalhamento é válida, uma vez que vamos escolher os estados iniciais e finais como sendo vácuo e a interação que ocorrerá entre eles vai ser computada pela matriz S. Esses são os ingredientes essenciais para uma descrição da dinâmica de um espalhamento.

Baseado na teoria de interação da teoria quântica de campos, esse método aparenta ser mais complexo que os outros utilizados, contudo permite uma visão clara dos limites em que os cálculos estão sendo feitos, característica comum dos sistemas na eletrodinâmica quântica com potencial externo clássico. Podemos, com isso, investigar a possibilidade de observação do fenômeno. Esse método, também, nos fornece uma interpretação do processo de criação de partículas via tunelamento.

A matriz S, ou matriz de espalhamento, também descreve o sistema de um campo clássico externo, posto que representa a dinâmica das flutuações do vácuo e sempre está presente em quaisquer cálculos de um efeito eletromagnético. A teoria da matriz S descreve somente efeitos quânticos do vácuo de absorção, ou seja, criação de partículas. Esse método se apresenta como uma outra maneira de quantificar os efeitos induzidos por um campo clássico externo em um sistema quântico.

Essencial para uma descrição precisa da evolução temporal de um espalhamento é a localização das partículas envolvidas no processo ou, no nosso caso, a determinação dos estados. Para a descrição típica de um espalhamento, como já vimos, necessitamos do seguinte arranjo. Primeiramente, temos um estágio inicial, em que o estado inicial é fixado assintoticamente longe do evento no qual o potencial age. Temos que definir também um estágio final, que ocorre após o espalhamento, em que o estado final não sofra influência do potencial espalhador. Ou seja, os estados inicial e final são estados livres. Temos que observar que quando trabalhamos nesse formalismo, nossos estados iniciais e finais são pré-definidos, não sendo possível estudar a evolução do estado.

Suponha que temos vácuo no estado inicial ( $i n$ ) e no estado final (out), e que o campo é ligado adiabaticamente em tempos finitos. Com isso os campos quânticos inicial e final são diferentes e, portanto, seus vácuos também serão diferentes, definidos como: 


$$
\left\{\begin{array}{l}
a_{\text {ink }}|0\rangle=0, b_{\text {ink }}|0\rangle=0 \\
a_{\text {out } \mathbf{k}}|0\rangle=0, b_{\text {out } \mathbf{k}}|0\rangle=0
\end{array} .\right.
$$

Os campos inicial e final são relacionados através da matriz $S$, ou matriz de espalhamento. Matematicamente, como esse conjunto inicial e final de estados é completo, existe uma transformação unitária, $S$, que relaciona esses estados in e out e contém toda a informação sobre a interação do sistema. Trabalhando no espaço de Fock das partículas incidentes, temos que:

$$
|0\rangle_{\text {in }}=S|0\rangle_{\text {out }}
$$

Na representação de Heisenberg, vemos que os campos in e out são relacionados da forma:

$$
\varphi_{\text {in }}=S \varphi_{\text {out }} S^{-1}
$$

A matriz $S$ é definida como sendo:

$$
S=\lim _{t \rightarrow+\infty} U(t)=T \exp \left[i \int d^{4} x \mathcal{L}_{\text {int }}(x)\right]
$$

em que $U(t)$ é o operador de evolução temporal e $T$ denota o produto ordenado temporalmente. Em toda teoria sem acoplamento derivativo, como é o caso, temos que:

$$
H_{\text {int }}(t)=\int d^{3} x \mathcal{H}_{\text {int }}=-\int d^{3} x \mathcal{L}_{\text {int }}
$$

então podemos escrever a matriz $\mathrm{S}$ também em termos da hamiltoniana de interação.

Com isso em mãos, definimos a probabilidade de não emitir pares:

$$
P_{0}=\left|\left\langle 0_{\text {out }} \mid 0_{\text {in }}\right\rangle\right|^{2}=\left|\left\langle 0_{\text {in }}|S| 0_{\text {in }}\right\rangle\right|^{2}=\left|S_{0}\right|^{2},
$$

em que $S_{0}$ é a amplitude de probabilidade de não emitir pares. Podemos ver aqui, comparando com as equações (A.16) e (A.17), que a matriz S se relaciona com a parte imaginária da Lagrangeana efetiva, ou seja, a matriz S computa efeitos de absorção. Logo, podemos ver a equivalência entre os métodos da Lagrangeana efetiva e da matriz $\mathrm{S}$ para a determinação do efeito Schwinger.

Vamos calcular, então, essa amplitude para ambos os campos escalar complexo e fermiônico

Vamos primeiramente pensar no campo escalar complexo. Pela definição dada no início da seção, definimos a matriz S como: 


$$
\begin{aligned}
S_{b} & =T \exp \left[i \int d^{4} x \mathcal{L}_{i n t}(x)\right] \\
& =T \exp \left\{\frac{i e}{2}\left[\left(\partial_{\mu} \phi A^{\mu} \phi^{*}-A_{\mu} \phi \partial^{\mu} \phi^{*}\right)+i e A^{2} \phi \phi^{*}\right]\right\},
\end{aligned}
$$

em que a utilizamos a Lagrangeana efetiva dada em (5.4) e denotamos a matriz S para os bósons com um índice subscrito $b$.

Estamos interessados em calcular a probabilidade vácuo-vácuo, ou seja, a probabilidade de não criar nenhum par. A amplitude de probabilidade associada a essa probabilidade é dada por:

$$
\begin{aligned}
S_{0, b}(A) & =\sum_{n=0}^{\infty} \frac{(i e)^{n}}{2^{n} n !} \int \prod_{i=1}^{n} d x_{i} \\
& \times\left\langle 0\left|T\left[\left(\partial_{\mu} \phi A^{\mu} \phi^{*}-A_{\mu} \phi \partial^{\mu} \phi^{*}\right)+i e A^{2} \phi \phi^{*}\right]\left(x_{1}\right)\right| 0\right\rangle \times \ldots \times \\
& \times\left\langle 0\left|T\left[\left(\left(\partial_{\mu} \phi\right) A^{\mu} \phi^{*}-A_{\mu} \phi\left(\partial^{\mu} \phi^{*}\right)\right)-i e A^{2} \phi \phi^{*}\right]\left(x_{n}\right)\right| 0\right\rangle .
\end{aligned}
$$

onde expandimos S. O motivo dessa expansão ficará claro posteriormente.

Pela equação acima, vemos que é interessante expressarmos a matriz S na ordem normal, uma vez que é uma forma mais conveniente para a determinação dos elementos de matriz da expansão no espaço de Fock. Utilizamos para isso o teorema de Wick para campos bosônicos, que é uma ferramenta que permite fazermos manipulações algébricas que reordenam o produto temporalmente ordenado para a ordem normal.

Com os elementos da expansão na ordem normal, é possível identificá-la como uma série de matrizes. Essa série pode ser identificada como sendo a fórmula para o inverso de um determinante, pelo teorema de Cailey-Hamilton inverso [111]. Assim, a amplitude de probabilidade $S_{0}$, ou amplitude vácuo-vácuo, pode ser escrita como:

$$
S_{0, b}(A)=\operatorname{Det}^{-1}\left(I-\Gamma_{b}\right)=\exp -\left[\operatorname{Tr} \ln \left(I-\Gamma_{b}\right)\right]
$$

onde o determinante e o traço são escritos com letras maiúsculas, pois envolvem integrações em variáveis contínuas. $\Gamma_{b}$ é associado aos elementos de matriz resultantes da expansão de Wick, e é dado por:

$$
\left\langle x\left|\Gamma_{b}\right| x\right\rangle=\underbrace{\left\langle 0_{i n}\left|T\left(\left(\partial_{\mu} \phi\right) A^{\mu} \phi^{*}-A_{\mu} \phi\left(\partial^{\mu} \phi^{*}\right)\right)\right| 0_{i n}\right\rangle}_{(i)}+i e \underbrace{A^{2}\left\langle 0_{i n}\left|T \phi \phi^{*}\right| 0_{i n}\right\rangle}_{(i i)} .
$$

Assim, em (ii) podemos identificar o propagador do campo escalar livre e podemos calcular $(i)$ substituindo a solução da equação de Klein-Gordon para os campos $\phi$ e $\phi^{*}$. 
Com isso, temos que:

$$
\Gamma_{b}=\frac{-e^{2} A^{2}+2 e P_{\mu} A^{\mu}}{P^{2}-m^{2}+i \varepsilon} \Rightarrow I-\Gamma_{b}=\frac{(P-e A)^{2}-m^{2}+i \varepsilon}{P^{2}-m^{2}+i \varepsilon}=\frac{G}{G_{0}},
$$

onde $G_{0}$ é o propagador do campo escalar livre e $G$ é o propagador do campo escalar complexo interagindo com um potencial clássico. Isso pode ser facilmente calculado das equações de movimento da Lagrangeana do sistema, vista em (5.3).

Logo, substituindo esses resultados, a amplitude vácuo-vácuo pode ser escrita como:

$$
\begin{aligned}
S_{0, b}(A) & =\operatorname{Det}^{-1}\left(\frac{(P-e A)^{2}-m^{2}+i \varepsilon}{P^{2}-m^{2}+i \varepsilon}\right) \\
& =\exp \left[-\operatorname{Tr} \frac{(P-e A)^{2}-m^{2}+i \varepsilon}{P^{2}-m^{2}+i \varepsilon}\right] .
\end{aligned}
$$

A forma da amplitude acima não é válida para qualquer campo externo, uma vez que o modelo não tem solução exata para um campo externo qualquer, mas somente em casos especiais como o de um campo elétrico constante.

Esses resultados podem facilmente ser estendidos para férmions. Da Lagrangeana de interação para o campo fermiônico (5.22), podemos escrever a matriz S da forma:

$$
S_{f}=T \exp \left[-i e \int d^{4} x \bar{\psi}(x) \gamma^{\mu} \psi(x) A_{\mu}(x)\right],
$$

em que o índice subscrito $f$ denota a matriz S para férmions. Assim, a amplitude vácuovácuo nesse caso é dada por:

$$
\begin{aligned}
S_{0, f}(A) & =\sum_{n=0}^{\infty} \frac{(-i e)^{n}}{n !} \int \prod_{i=1}^{n} d x_{i} \\
& \times\left\langle 0_{i n}\left|\bar{\psi}\left(x_{1}\right) \gamma^{\mu} \psi\left(x_{1}\right) A_{\mu}\left(x_{1}\right) \ldots \bar{\psi}\left(x_{n}\right) \gamma^{\mu} \psi\left(x_{n}\right) A_{\mu}\left(x_{n}\right)\right| 0_{i n}\right\rangle,
\end{aligned}
$$

Fazendo o mesmo procedimento acima, queremos colocar essa expansão na ordem normal. Utilizamos novamente o teorema de Wick, só que desta vez para férmions. Pelo teorema de Cailey-Hamilton, escrevemos a série como um determinante $S_{0, f}(A)=$ $\operatorname{Det}\left[I-\Gamma_{f}\right]$, em que determinamos $\Gamma_{f}=e \gamma_{\mu} A^{\mu}(x) \frac{1}{\gamma_{\mu} P^{\mu}-m+i \varepsilon}$, uma vez que os elementos de matriz da expansão correspondiam aos propagadores de Feynmann livre do campo de fermiônico, a saber $\left\langle 0\left|{\overline{\alpha_{k}}}^{-}\left(x_{k}\right) \psi_{\alpha_{l}}\left(x_{l}\right)\right| 0\right\rangle=i S_{F}(x-y)_{\alpha_{k} \alpha_{l}}$. Assim, a amplitude vácuovácuo para o campo fermiônico é dada por: 


$$
\begin{aligned}
S_{0, f}(A) & =\operatorname{Det}\left[I-e \gamma_{\mu} A^{\mu}(x) \frac{1}{\gamma_{\mu} P^{\mu}-m+i \varepsilon}\right] \\
& =\exp \left[-\operatorname{Tr} \ln \left(\left[\gamma_{\mu} P^{\mu}-m\right] \frac{1}{\gamma_{\mu} P^{\mu}-e \gamma_{\mu} A^{\mu}(x)-m+i \varepsilon}\right)\right] .
\end{aligned}
$$

Assim como no caso do campo escalar, a forma da amplitude acima não é válida para qualquer campo externo, uma vez que o modelo não tem solução exata para um campo externo qualquer.

Como não podemos utilizar as amplitudes de probabilidade (5.46) e (5.49) para o cálculo para um potencial qualquer, vamos colocar essas amplitudes em uma forma mais geral.

Uma outra maneira de descrever um problema de espalhamento é por meio de uma equação de Lippmann-Schwinger [107]. Essa equação integral determina os estados assintóticos do problema, escritos em função do operador de transição de um corpo, ou operador T. A matriz $\mathrm{S}$ também pode ser escrita em função desses operadores T. Para isso, vamos utilizar as conseqüências da unitariedade da matriz S.

Para isso, introduzimos o operador de espalhamento de um corpo, que pode ser deduzido da equação de Lippmann Schwinger, definido como sendo:

$$
\begin{gathered}
\mathcal{T}_{b}(A)=V+V \frac{1}{P^{2}-m^{2}+i \varepsilon} \mathcal{T}_{b}(A), \\
\overline{\mathcal{T}}_{b}(A)=V+V \frac{1}{P^{2}-m^{2}-i \varepsilon} \overline{\mathcal{T}}_{b}(A),
\end{gathered}
$$

onde $V=\frac{1}{G_{0}}-\frac{1}{G}$ e $\overline{\mathcal{T}}_{b}(A)=\mathcal{T}_{b}^{\dagger}(A)$. Para o campo fermiônico, uma vez que $\overline{\mathcal{T}}_{f}=\gamma_{0} \mathcal{T}_{f}^{\dagger} \gamma_{0}$, esse operador é definido da forma:

$$
\begin{gathered}
\mathcal{T}_{f}(A)=e \gamma_{\mu} A^{\mu}(x)\left[1+\frac{\mathcal{T}_{f}(A)}{\gamma_{\mu} P^{\mu}-m+i \varepsilon}\right] \\
\overline{\mathcal{T}}_{f}(A)=e \gamma_{\mu} A^{\mu}(x)\left[1+\frac{\overline{\mathcal{T}}_{f}(A)}{\gamma_{\mu} P^{\mu}-m-i \varepsilon}\right]
\end{gathered}
$$

Operando com esses propagadores, temos que:

$$
\mathcal{T}(A)-\overline{\mathcal{T}}(A)=i\left(\rho^{(+)}+\rho^{(-)}\right),
$$

com isso, definimos os operadores de densidade de estado: 


$$
\begin{aligned}
\rho_{b}^{( \pm)} & \equiv 2 \pi \theta\left( \pm P^{0}\right) \delta\left(P^{2}-m^{2}\right) \\
\rho_{f}^{( \pm)} & \equiv 2 \pi\left[\gamma_{\mu} P^{\mu}+m\right] \theta\left( \pm P^{0}\right) \delta\left(P^{2}-m^{2}\right)
\end{aligned}
$$

que são elementos que projetam na concha de massa.

Os termos da expansão de Wick podem ser decompostos em ciclos, uma vez que são uma soma de todas as possíveis permutações. Se tivéssemos utilizado em nossos cálculos o propagador retardado ao invés do propagador livre, todos esses termos cíclicos se anulariam, dado que o propagador retardado é nulo no cone de luz futuro, assim como os termos cíclicos. Com isso temos que $\Gamma=0$. Com o propagador retardado, $G_{r}=\frac{1}{P^{2}-m^{2}+i \varepsilon}$, temos que:

$$
\operatorname{Det}\left(\frac{(P-e A)^{2}-m^{2}+i \varepsilon}{P^{2}-m^{2}+i \varepsilon}\right)=1 \text {. }
$$

E no caso fermiônico, $S_{r}=\frac{\gamma_{\mu} P^{\mu}+m}{(P+i \varepsilon)^{2}-m^{2}}=\frac{1}{\gamma_{\mu} P^{\mu}-m-i \varepsilon}$, logo

$$
\operatorname{Det}\left(I-e \gamma_{\mu} A^{\mu}(x) \frac{1}{\gamma_{\mu} P^{\mu}-m-i \varepsilon}\right)=1 \text {. }
$$

Manipulando essa expressão, utilizando a unitariedade de S e a equação (5.43) podemos escrever $\left|S_{0}(A)\right|^{2}$ como:

$$
\left|S_{0}(A)\right|^{2}=\exp \left\{\operatorname{Tr} \ln \left[I-\mathcal{T}(\mathcal{A}) \rho^{(+)} \overline{\mathcal{T}}(A) \rho^{(-)}\right]\right\},
$$

lembrando que o Tr é escrito em letras maiúsculas, pois envolve integrações em variáveis contínuas.

Uma vez que a probabilidade de não emitir pares pode ser escrita como $P_{0}=\mathrm{e}^{\int d^{4} x \omega}=$ $\mathrm{e}^{V T \omega}$, em que $V$ é o volume, $T$ é o tempo e $\omega$ é a taxa de criação de partículas, temos que essa taxa é dada por:

$$
\begin{gathered}
\omega_{B}(x)=\left\langle x\left|\ln \left[I-\mathcal{T}(\mathcal{A}) \rho^{(+)} \overline{\mathcal{T}}(A) \rho^{(-)}\right]\right| x\right\rangle, \\
\omega_{F}(x)=\operatorname{tr}\left\langle x\left|\ln \left(I-\mathcal{T}(\mathcal{A}) \rho^{(+)} \overline{\mathcal{T}}(A) \rho^{(-)}\right)\right| x\right\rangle,
\end{gathered}
$$

onde esse traço é tomado sobre os índices de Dirac e $\omega_{b}(x)$ e $\omega_{F}(x)$ são densidades de probabilidade de criação de pares, ou probabilidades por unidade de tempo e por unidade de volume dos bósons e dos férmions, respectivamente. Essa expressão é a forma geral da probabilidade de não emitir pares e pode ser utilizada para quaisquer potenciais eletromagnéticos. Vamos utilizá-las no capítulo 4, quando o campo elétrico é variável no tempo. 


\subsubsection{Campo elétrico constante}

Agora, queremos calcular a taxa de criação de pares elétron-pósitron no caso de um campo elétrico uniforme. Para esse caso, podemos utilizar diretamente as equações (5.46) e (5.49) uma vez que é um modelo exatamente solúvel e é o modelo de interesse para nós (o efeito Schwinger).

A partir de (5.46) e (5.49), queremos calcular a probabilidade de não emitir pares. Assim, para bósons, aplicando o logaritmo e sabendo que o traço é invariante por transposição, temos que:

$$
\left|S_{0}(A)\right|^{2}=\exp \int d^{4} x \underbrace{\operatorname{tr}\left\langle x\left|\ln \left(\frac{\left(P^{2}-m^{2}+i \varepsilon\right)^{2}}{\left[(P-e A)^{2}-m^{2}+i \varepsilon\right]}\right)\right| x\right\rangle}_{\omega_{B}(x)} .
$$

Vamos utilizar o método do tempo próprio para computar o logaritmo acima. Nesse método podemos escrever o logaritmo na forma:

$$
\ln \hat{D}=-\int_{0}^{\infty} \frac{d s}{s} \exp [-i(\hat{D}-i \varepsilon) s] .
$$

Considerando apenas a parte real da solução, que é a parte física, $\omega_{B}$ é dado como:

$$
\omega_{B}(x)=-2 \operatorname{Re} \int_{0}^{\infty} \frac{d s}{s} e^{-i s\left(m^{2}\right)}\left\langle x\left|\exp \left\{i s\left[[P-e A(x)]^{2}-P^{2}\right]\right\}\right| x\right\rangle .
$$

Para o campo fermiônico, podemos fazer o mesmo procedimento através de (5.49) para obtermos $\omega_{F}$ em função do parâmetro de tempo próprio:

$$
\begin{aligned}
\omega_{F}(x) & =\operatorname{Re} \int_{0}^{\infty} \frac{d s}{s} e^{-i s\left(m^{2}\right)} \\
& \times \operatorname{tr}\left(\left\langle x\left|\exp \left\{i s\left[[P-e A(x)]^{2}+\frac{e}{2} \sigma_{\mu \nu} F^{\mu \nu}-P^{2}\right]\right\}\right| x\right\rangle\right),
\end{aligned}
$$

uma vez que $[P-e A(x)]^{2}+\frac{e}{2} \sigma_{\mu \nu} F^{\mu \nu}$ resulta de multiplicarmos a equação de Dirac pelo operador $\left(i \gamma_{\mu} \partial^{\mu}-e \gamma_{\mu} A^{\mu}+m\right)$.

Vamos calcular a taxa de criação de pares para um campo puramente elétrico constante. O campo tem que ser puramente elétrico uma vez que é a aceleração devido ao campo elétrico que possibilita às partículas tunelarem pela barreira de potencial $2 \mathrm{~m}$. Já um campo puramente magnético não cria partículas - por isso a condição covariante $\mathbf{E}^{2}-\mathbf{B}^{2}>0$ para que haja criação de pares.

Escolhendo o campo elétrico contante na direção z: 


$$
\mathbf{E}=E \hat{k}
$$

assim, o gauge escolhido é tal que:

$$
A^{3}(x)=-E t
$$

é a única componente não nula.

Para determinarmos a probabilidade $\omega$, temos que calcular o traço em (5.62) e em (5.63). Primeiramente, vamos separar as componentes longitudinais e transversais. O traço para bósons e para férmions é praticamente o mesmo, diferindo apenas por um termo extra proporcional ao tensor eletromagnético para férmions. Assim, no caso dos férmions:

$$
\begin{aligned}
\operatorname{tr}\left(\left\langle x\left|e^{i s\left[[P-e A(x)]^{2}+\frac{e}{2} \sigma_{\mu \nu} F^{\mu \nu}\right]}\right| x\right\rangle\right) & =4 \cosh (e E s) \int \frac{d^{4} p}{(2 \pi)^{4}} \frac{d^{4} k}{(2 \pi)^{4}} e^{i(p-k) x} \\
& \times e^{\left(-i \frac{P^{0} P^{3}}{e E}\right)}\left\langle p\left|e^{i s\left(P_{0}^{2}-\overrightarrow{P_{T}^{2}}-e^{2} E^{2} X_{0}^{2}\right)}\right| k\right\rangle e^{\left(i \frac{P^{0} P^{3}}{e E}\right)} \\
& =\frac{2 e E}{(2 \pi)^{2}} \cosh (e E s) \underbrace{\int d \omega\left\langle\omega\left|e^{i s\left(P_{0}^{2}-e^{2} E^{2} X_{0}^{2}\right)}\right| \omega\right\rangle}_{(I)},
\end{aligned}
$$

em que, usando $\left[X_{0}, P_{0}\right]=-i$ e da fórmula de Baker-Hausdorff, escrevemos:

$$
[P-e A(x)]^{2}+\frac{e}{2} \sigma_{\mu \nu} F^{\mu \nu}=e^{\left(-i \frac{P_{0} P_{3}}{e E}\right)}\left[P_{0}^{2}-P_{\perp}^{2}-e^{2} E^{2} t^{2}\right] e^{\left(i \frac{P_{0} P_{3}}{e E}\right)}+\frac{e}{2} \sigma_{\mu \nu} F^{\mu \nu},
$$

sendo que para o caso bosônico temos a mesma expressão para o traço, desconsideramos o termo proporcional ao tensor eletromagnético

Para podermos resolver a integral (I) acima, vamos fazer uma analogia com o oscilador harmônico. A integral acima pode ser considerada como o traço do operador de evolução de um oscilador harmônico com freqüência $i \omega$. Podemos ver isso se fizermos a correspondência:

$$
\begin{aligned}
P_{0} & \rightarrow P \\
-X_{0} & \rightarrow Q \\
2 i e E & \rightarrow \omega_{0} \\
1 / 2 & \rightarrow m_{0} \\
\Rightarrow P_{0}^{2}-e^{2} E^{2} X_{0}^{2} & \rightarrow P^{2}+\frac{\omega_{0}^{2}}{4} Q^{2}=\frac{P^{2}}{2 m_{0}^{2}}+\frac{m \omega_{0}^{2}}{2} Q^{2} .
\end{aligned}
$$


Assim, temos que os níveis de energias do oscilador harmônico nos fornecem:

$$
\begin{aligned}
\int d \omega\left\langle\omega\left|e^{i s\left(P_{0}^{2}-e^{2} E^{2} X_{0}^{2}\right)}\right| \omega\right\rangle & =\operatorname{Tr} \exp \left[i s\left(\frac{P^{2}}{2 m_{0}^{2}}+\frac{m_{0} \omega_{0}^{2}}{2} Q^{2}\right)\right] \\
& =\sum_{n=0}^{\infty} \exp [-e E s(2 n+1)] \\
& =\frac{1}{2} \frac{1}{\operatorname{senh}(e E s)} .
\end{aligned}
$$

Com isso, podemos escrever a taxa de criação como:

$$
\begin{gathered}
\omega_{B}(x)=\frac{1}{2(2 \pi)^{2}} \int_{-\infty}^{+\infty} \frac{d s}{s^{2}}\left[e E \frac{1}{\operatorname{senh}(e E s)}-\frac{1}{s}\right] \operatorname{Re}\left[i e^{-i s m^{2}}\right] \\
\omega_{F}(x)=-\frac{1}{2(2 \pi)^{2}} \int_{-\infty}^{+\infty} \frac{d s}{s^{2}}\left[e E \operatorname{coth}(e E s)-\frac{1}{s}\right] \operatorname{Re}\left[i e^{-i s m^{2}}\right]
\end{gathered}
$$

em que o termo $1 / \mathrm{s}$ corresponde à subtração do termo com $e=0$ em $(5.62) \mathrm{e}$, como o integrando é par, escrevemos a integral de $-\infty$ a $+\infty$ como sendo metade da integral de 0 a $\infty$.

Calculando essa integral complexa em um caminho que engloba o eixo imaginário negativo, temos que a taxa de criação de partículas é dada por:

$$
\omega_{B}=\frac{\alpha E^{2}}{2 \pi^{2}} \sum_{n=1}^{\infty} \frac{(-1)^{n+1}}{n^{2}} \exp \left(-\frac{n \pi m^{2}}{|e E|}\right),
$$

para bósons, e para férmions:

$$
\omega_{F}=\frac{\alpha E^{2}}{\pi^{2}} \sum_{n=1}^{\infty} \frac{1}{n^{2}} \exp \left(-\frac{n \pi m^{2}}{|e E|}\right) .
$$

Podemos ver que esse resultado sempre é maior do que zero mas menor do que um, ou seja, há uma probabilidade não-nula de criação de partículas. Tanto a fórmula (5.78) do caso fermiônico, quanto a fórmula (5.77) no caso bosônico apresentam o principal fator: $\exp \left(-\frac{n \pi m^{2}}{|e E|}\right)$, um termo não perturbativo no campo elétrico externo. Isso vem do fenômeno de tunelamento pela barreira de potencial $2 \mathrm{~m}$. A aceleração devido ao campo elétrico permite que as partículas tunelem a barreira de potencial. Como um campo magnético constante puro não pode transferir energia para uma partícula carregada, temos que campos magnéticos não criam partículas.

Notemos que as expressões finais da probabilidade de criação de pares, por unidade de volume e por unidade de tempo, para o caso fermiônico (5.78) e bosônico (5.77), diferenciam, essencialmente, por um fator dois, proveniente do grau de liberdade de spin. 
Vemos também que $\omega_{B}$ é uma série alternada. Isso indica que termos sucessivos dessa expansão não podem ser interpretados como a probabilidade de criação de $1,2, \ldots, \mathrm{n}$ pares, como pode ser feito com $\omega_{F}$, do caso fermiônico.

Podemos verificar que, para que o efeito seja observado, são necessários campos elétricos estáticos com energia da ordem de $E_{\text {crit }} \sim 1.3 \times 10^{18} \mathrm{~V} / \mathrm{m}$. Contudo, como tipicamente $|e E| \ll m^{2}$ nos aparatos experimentais existentes, a taxa de criação é muito pequena e como conseqüência esse fenômeno (o efeito Schwinger estático) nunca foi observado.

\subsubsection{Dinâmica via transformadas de Bogoliubov}

Nesta Seção apresentaremos mais um método baseado no cálculo da matriz S. Aqui iremos descrever a dinâmica da criação de partículas via o método dos modos normais, ou das transformações de Bogoliubov. A transformação de Bogoliubov descreve o sistema em seus modos normais, ou seja, diagonaliza a hamiltoniana em seus novos operadores de criação e aniquilação. O uso das transformações de Bogoliubov é um método muito bem estabelecido de calcular a matriz S. Esse método tem a vantagem, em relação aos outros apresentados, de ser mais automático e mais facilmente adaptável para diferentes formas do campo externo aplicado. Essa característica faz com que essa descrição seja muito útil também para a comparação do caso eletromagnético com seu análogo cosmológico.

As transformações de Bogoliubov foram desenvolvidas por N. N. Bogoliubov em 1958 [108] para tratar problemas de supercondutividade. Contudo seu uso na mecânica quântica e na TQC foi rapidamente reconhecido e é utilizado como um poderoso método de diagonalização de Hamiltonianas. Seu análogo dependente do tempo também tem sido muito utilizado em problemas com campos externos, como fazemos aqui.

A descrição via transformadas de Bogoliubov visa computar a transição entre os estados nos quais ocorreu a interação, ou seja, entre os quais o campo externo agiu. Assim, como na descrição via teoria de espalhamento, temos que definir os estados inicial e final do problema. Vamos definir os estados in e out como sendo aqueles em que o campo externo ainda não agiu e já deixou de agir, respectivamente. Convém salientar que os estados in e out, como definidos nas seções anteriores, não habitam dois espaços de Hilbert distintos, mas sim dois espaços de Fock distintos. Logo, qualquer estado in pode ser escrito como uma combinação de estados out, e vice-versa, por meio de uma transformação canônica, uma vez que os estados in e out são conjuntos de estados ortonormais. Os coeficientes que ligam esses estados são os coeficientes de Bogoliubov e definem a matriz S. A teoria de Bogoliubov aparece como uma uma ferramenta importante que permite que a teoria seja escrita como uma evolução de um estado inicial definido.

Esse conceito de estados assintóticos é importante também quando nos deparamos com o conceito de partícula, pois este apresenta uma ambigüidade nos instantes em que a sua taxa de criação não é desprezível, ou seja, quando o campo está agindo. Com isso 
a noção de partícula nesses instantes não é bem definida. Contudo, o vácuo adiabático é um bom vácuo para resolver esses problemas que não são assintoticamente estáveis, uma vez que o conceito de partícula nele é bem definido. Com isso vamos utilizar o vácuo adiabático como os estados assintóticos infinitos, in e out, entre os quais o processo de criação de partículas ocorre.

Uma vantagem do cálculo utilizando transformadas de Bogoliubov é que também podemos estudar a evolução do estado inicial, ou seja, sem termos a necessidade de fixarmos, como feito nas descrições anteriores, o estado final. Com isso, podemos calcular o número de partículas criadas após a interação com o campo externo. Contudo, o estado final também deve ser um estado adiabático, mesmo que não seja o vácuo.

\subsubsection{Número de partículas criadas}

Como nos estados inicial e final não temos interação com o campo externo, as funções de onda que descrevem esses estados são a soluções da equação de Klein Gordon livre, $\phi_{\text {in }}$ e $\phi_{\text {out }}$, que estão definidas no conjunto completo ortonormal de estados in e out, respectivamente [109]. Assim, estas soluções também formam um conjunto completo ortonormal de soluções definida em $t \rightarrow-\infty$ e $t \rightarrow+\infty$, respectivamente. Sendo um conjunto completo de soluções ortogonais, podemos escrever uma como combinação linear da outra, ou seja, podemos fazer uma transformação conectando esses estados:

$$
\left\{\begin{array} { l } 
{ \phi _ { \text { in } } = \alpha _ { b } \phi _ { \text { out } } + \beta _ { b } \phi _ { \text { out } } ^ { \dagger } } \\
{ \phi _ { \text { in } } ^ { \dagger } = \beta _ { b } ^ { * } \phi _ { \text { out } } + \alpha _ { b } ^ { * } \phi _ { \text { out } } ^ { \dagger } }
\end{array} \quad \left\{\begin{array}{l}
\phi_{\text {out }}=\alpha_{b}^{*} \phi_{\text {in }}-\beta_{b} \phi_{\text {out }}^{\dagger} \\
\phi_{\text {out }}^{\dagger}=-\beta_{b}^{*} \phi_{\text {out }}+\alpha_{b} \phi_{\text {out }}^{\dagger}
\end{array} .\right.\right.
$$

Das relações de comutação (5.14), impostas na quantização canônica, podemos ver que, se $\mathbf{x}=\mathbf{y}$, temos que:

$$
\left|\alpha_{b}\right|^{2}-\left|\beta_{b}\right|^{2}=1
$$

que são as condições de Bogoliubov para o campo escalar.

Analogamente para o campo fermiônico, podemos definir uma transformação de Bogoliubov entre os estados assintóticos $\psi_{\text {in }}$ e $\psi_{\text {out }}$, soluções da equação de Dirac livre:

$$
\left\{\begin{array} { l } 
{ \psi _ { \text { in } } = \alpha _ { f } \psi _ { \text { out } } + \beta _ { f } \overline { \psi } _ { \text { out } } } \\
{ \overline { \psi } _ { \text { in } } = \beta _ { f } ^ { * } \psi _ { \text { out } } + \alpha _ { f } ^ { * } \overline { \psi } _ { \text { out } } }
\end{array} \quad \left\{\begin{array}{l}
\psi_{\text {out }}=\alpha_{f}^{*} \psi_{\text {in }}-\beta_{f} \bar{\psi}_{\text {out }} \\
\bar{\psi}_{\text {out }}=-\beta_{f}^{*} \psi_{\text {out }}+\alpha_{f} \bar{\psi}_{\text {out }}
\end{array}\right.\right.
$$

E nesse caso, de acordo com as regras de anticomutação (5.31), temos que as condições de Bogoliubov são dadas por:

$$
\left|\alpha_{n}\right|^{2}+\left|\beta_{n}\right|^{2}=1
$$

Podemos também obter a transformação de Bogoliubov para os operadores de criação 
e aniquilação. De acordo com a decomposição (5.7) e (5.24), para bósons e férmions, podemos escrevê-las da forma:

$$
\begin{gathered}
\phi_{i n}(x)=\frac{1}{(2 \pi)^{3}} \int \frac{d^{3} \mathbf{k}}{\sqrt{2 \omega_{\mathbf{k}}}}\left(A_{\mathbf{k}} e^{-i \mathbf{k x}}+B_{\mathbf{k}}^{*} e^{i \mathbf{k x}}\right), \\
\psi_{\text {in }}(x)=\frac{1}{(2 \pi)^{3}} \int \frac{d^{3} k}{\omega_{k} / m} \sum_{r=1,2}[D_{r}(k) \underbrace{u^{(r)}(k) \mathrm{e}^{-i k \cdot x}}_{\psi^{(+)}}+F_{r}^{\dagger}(k) \underbrace{v^{(r)}(k) \mathrm{e}^{i k . x}}_{\psi^{(-)}}],
\end{gathered}
$$

em que $A_{k}, A_{k}^{\dagger}, B_{k}$ e $B_{k}^{\dagger}$ são os operadores de criação e aniquilação de partículas e antipartículas, e $D_{r}(k), D_{r}^{\dagger}(k), F_{r}(k)$ e $F_{r}^{\dagger}(k)$, analogamente para o campo fermiônico.

Esses operadores estão definidos para $t \rightarrow-\infty$, isto é, antes do campo externo ter sido ligado, e definem o vácuo in:

$$
\begin{aligned}
& A_{k}\left|0_{i n}\right\rangle=D_{r}(k)\left|0_{i n}\right\rangle=0 \\
& B_{k}\left|0_{i n}\right\rangle=F_{r}(k)\left|0_{i n}\right\rangle=0
\end{aligned} .
$$

Fica claro que para os estados out também temos a mesma decomposição. Contudo, os operadores de criação e aniquilação da decomposição do estado out, a saber $a_{k}, a_{k}^{\dagger}, b_{k}$ e $b_{k}^{\dagger}$ para bósons e $f_{r}(k), d_{r}^{\dagger}(k), f_{r}(k)$ e $b_{r}^{\dagger}(k)$ para férmions, não agem de forma igual no estado in, ou seja:

$$
\begin{aligned}
& a_{k}\left|0_{i n}\right\rangle \neq 0, \quad d_{r}(k)\left|0_{i n}\right\rangle \neq 0, \\
& b_{k}\left|0_{i n}\right\rangle \neq 0, \quad f_{r}(k)\left|0_{i n}\right\rangle \neq 0 .
\end{aligned}
$$

Isso pode ser visto uma vez que, a partir das decomposições dos campos, fica claro que podemos também relacionar os operadores de criação e aniquilação por uma transformada de Bogoliubov:

$$
\left\{\begin{array} { l } 
{ A _ { k } = x _ { 1 k } a _ { k } + x _ { 2 k } b _ { k } ^ { \dagger } } \\
{ B _ { k } ^ { \dagger } = x _ { 2 k } ^ { * } a _ { k } + x _ { 1 k } ^ { * } b _ { k } ^ { \dagger } }
\end{array} \quad \left\{\begin{array}{l}
a_{k}=x_{1 k}^{*} A_{k}+-x_{2 k} B_{k}^{\dagger} \\
b_{k}^{\dagger}=-x_{2 k}^{*} A_{k}+x_{1 k} B_{k}^{\dagger}
\end{array}\right.\right.
$$

e, omitindo o índice espinorial, para férmions:

$$
\left\{\begin{array} { l } 
{ D _ { k } = y _ { 1 k } d _ { k } + y _ { 2 k } f _ { k } ^ { \dagger } } \\
{ F _ { k } ^ { \dagger } = y _ { 2 k } ^ { * } d + y _ { 1 k } ^ { * } f _ { k } ^ { \dagger } }
\end{array} \quad \left\{\begin{array}{l}
d_{k}=y_{1 k}^{*} D_{k}-y_{2 k} F_{k}^{\dagger} \\
f_{k}^{\dagger}=-y_{2 k}^{*} D_{k}+y_{1 k} F_{k}^{\dagger}
\end{array}\right.\right.
$$

De forma a satisfazer as relação de comutação (5.16) e anticomutação (5.33), com $\mathbf{k}=\mathbf{k}^{\prime}$, para os operadores de criação e aniquilação, vemos que as condições de Bogoliubov são as mesmas que (5.80) e (5.82), respectivamente : 


$$
\begin{gathered}
\left|x_{1 k}\right|^{2}-\left|x_{2 k}\right|^{2}=1 . \\
\left|y_{1 k}\right|^{2}+\left|y_{2 k}\right|^{2}=1 .
\end{gathered}
$$

Assim, temos duas transformações de Bogoliubov que conectam os operadores de campo e os operadores de criação e aniquilação. Os coeficientes dessas transformações são relacionados da seguinte maneira:

$$
\left\{\begin{array} { l } 
{ x _ { 1 \mathbf { k } } ^ { * } = \alpha _ { \mathrm { b } } } \\
{ x _ { 2 \mathbf { k } } = \beta _ { \mathrm { b } } ^ { * } }
\end{array} \quad \text { e } \left\{\begin{array}{l}
y_{1 \mathbf{k}}^{*}=\alpha_{f} \\
y_{2 \mathbf{k}}=\beta_{f}
\end{array}\right.\right.
$$

Podemos ver que, se substituirmos a transformação de Bogoliubov nas equações de Hamilton, obteremos equações de Hamilton para os coeficientes de Bogoliubov:

$$
\begin{aligned}
i \dot{x}_{1 k} & =\omega_{k} x_{1 k}+i \frac{1}{2} \frac{\dot{\omega}_{k}}{\omega_{k}} x_{2 k} \\
i \dot{x}_{2 k} & =-\omega_{k} x_{2 k}+i \frac{1}{2} \frac{\dot{\omega}_{k}}{\omega_{k}} x_{1 k} .
\end{aligned}
$$

Assim, de posse dessa transformação podemos calcular o número médio de partículas criadas em função do tempo. Para as partículas bosônicas, temos que:

$$
\left\langle N_{\mathbf{k}}(t)\right\rangle=\left\langle 0\left|a_{\mathbf{k}}^{\dagger}(t) a_{\mathbf{k}}(t)\right| 0\right\rangle=\left\{\begin{array}{l}
\left|\beta_{\mathbf{b}}\right|^{2}=\left\langle N_{\mathbf{k}}(t)\right\rangle_{0}, \text { se }\left\langle N_{\mathbf{k}}\left(t_{1}\right)\right\rangle=0 \\
\left\langle N_{\mathbf{k}}\left(t_{1}\right)\right\rangle+\left|\beta_{\mathbf{b}}\right|^{2}\left(1+2\left\langle N_{\mathbf{k}}\left(t_{1}\right)\right\rangle\right) \text {, se }\left\langle N_{\mathbf{k}}\left(t_{1}\right)\right\rangle \neq 0
\end{array}\right.
$$

sendo que $\left\langle N_{\mathbf{k}}\left(t_{1}\right)\right\rangle$ é o número médio de partículas no estado inicial. O mesmo número de antipartículas é criado, uma vez que são criados pares. Dizemos que, na primeira equação de (5.94), houve uma produção espontânea de partículas e na segunda de produção estimulada.

Para as partículas fermiônicas:

$$
\left\langle N_{\mathbf{k}}(t)\right\rangle=\left\langle 0\left|a_{\mathbf{k}}^{\dagger}(t) a_{\mathbf{k}}(t)\right| 0\right\rangle=\left\{\begin{array}{c}
2\left|\beta_{\mathrm{f}}\right|^{2}=\left\langle N_{\mathbf{k}}(t)\right\rangle_{0}, \text { se }\left\langle N_{\mathbf{k}}\left(t_{1}\right)\right\rangle=0 \\
\left\langle N_{\mathbf{k}}\left(t_{1}\right)\right\rangle+\left|\beta_{\mathrm{f}}\right|^{2}\left(1-\left\langle N_{\mathbf{k}}\left(t_{1}\right)\right\rangle-\left\langle\bar{N}_{\mathbf{k}}\left(t_{1}\right)\right\rangle\right) \\
\text { se }\left\langle N_{\mathbf{k}}\left(t_{1}\right)\right\rangle \neq 0
\end{array}\right.
$$

sendo que $\left\langle N_{\mathbf{k}}\left(t_{1}\right)\right\rangle$ é o número médio de partículas no estado inicial e $\left\langle\bar{N}_{\mathbf{k}}\left(t_{1}\right)\right\rangle$ é o 
número de antipartículas no estado inicial. Dizemos que na primeira equação de (5.95) houve uma produção espontânea de partículas e na segunda, de produção desestimulada. A presença de férmions no estado inicial diminui o número de partículas criadas, dado que, pelo princípio de exclusão de Pauli, a presença de férmions no estado inicial deixa menos estados livres para serem ocupados.

Esse estado inicial de partículas (antipartículas) é um auto-estado do operador número. O caso de um estado inicial povoado mais realista, em que temos que considerar efeitos térmicos, pode ser visto em [112].

O número total de partículas criadas, então, é dado por:

$$
\langle N(t)\rangle=\frac{1}{(2 \pi)^{3}} \int d^{3} k\left\langle N_{\mathbf{k}}(t)\right\rangle
$$

\subsubsection{Probabilidade de emissão de pares}

Agora vamos calcular a probabilidade de não emitir pares [84]. Para isso, estaremos trabalhando na representação funcional de Schrödinger, em que faremos uso dos funcionais de onda. Vamos inicialmente introduzir um pouco desse formalismo.

Faremos esse procedimento somente para o campo escalar, que é de nosso interesse para a analogia.

Para passarmos para a representação funcional de Schrödinger, temos que as regras de comutação (5.14) admitem a representação:

$$
\left\{\hat{\phi} \Psi=\phi \Psi, \quad \hat{p} \Psi=-i \frac{\partial \Psi}{\partial \phi},\right.
$$

que corresponde à substituição $\hat{\phi} \rightarrow \phi$ e $\hat{p} \rightarrow-i \frac{\partial}{\partial \phi} ;$ e $\hat{\phi}^{\dagger} \rightarrow \phi^{*}$ e $\hat{p}^{\dagger} \rightarrow-i \frac{\partial}{\partial \phi^{*}}$; e $|\phi\rangle \rightarrow \Psi\left[\phi, \phi^{*}, t\right]$. Ou seja, agora o estado do sistema é descrito por um funcional do campo escalar. Nessa representação o produto interno é definido como:

$$
\langle\Psi \mid \Phi\rangle=\int \mathcal{D}^{2} \phi \Psi\left(\phi, \phi^{*}\right)^{*} \Phi\left(\phi, \phi^{*}\right)
$$

em que $\mathcal{D}^{2} \phi=\mathcal{D} \phi \mathcal{D} \phi^{*}$.

Esse funcional que representa o estado em nosso formalismo pode ser visto como uma superposição entre um estado geral e esse "estado de base de campo":

$$
\Psi\left(\phi, \phi^{*}\right)=\left\langle\phi, \phi^{*} \mid \Psi\right\rangle
$$

Assim, determinadas as características da nossa representação, partimos para a descrição do nosso sistema. A Hamiltoniana de nosso sistema, (5.12), é quadrática. Os 
auto-estados de qualquer Hamiltoniana quadrática podem ser escritos como estados gaussianos [109]. Logo, podemos fazer o ansatz:

$$
\Psi\left[\phi, \phi^{*}, t\right]=N(t) \exp \left(-\int d^{3} x d^{3} x^{\prime} \phi^{*}(x) \Omega\left(x, x^{\prime} ; t\right) \phi\left(x^{\prime}\right)\right),
$$

em que $\Omega\left(x, x^{\prime} ; t\right)$ é usualmente chamada de covariância. ${ }^{1}$

Esse ansatz é essencial para estudarmos a probabilidade de não emitir pares, pois estados gaussianos representam estados de vácuo na teoria quântica de campos. Ou seja, a evolução desse estado gaussiano sob a influência de um campo externo é o mesmo que calcularmos a evolução do vácuo sob a mesma influência. Logo, com isso, podemos calcular $\left\langle_{\text {out }} 0 \mid 0_{\text {in }}\right\rangle$.

Portanto, queremos estudar a evolução desse estado gaussiano em relação à equação de Schrödinger, que nessa representação é uma equação diferencial funcional, dada por:

$$
\begin{aligned}
i \frac{d \Psi}{d t}=H \Psi & \\
=\int d^{3} x & {\left[-\frac{\partial}{\partial \phi} \frac{\partial}{\partial \phi^{*}}+\left(\partial_{i} \phi^{*}\right)\left(\partial^{i} \phi\right)+i e_{b} A^{i}\left(\phi^{*} \overleftrightarrow{\partial}_{i} \phi\right)+\left(m^{2}-e_{b}^{2} A^{2} \phi^{*} \phi\right)\right] } \\
& \times \Psi\left[\phi, \phi^{*}, t\right]
\end{aligned}
$$

As funções $N(t)$ e $\Omega$ podem ser determinadas inserindo o ansatz (5.100) na equação de Shrödinger. Com isso, temos:

$$
\begin{aligned}
i \frac{\dot{N}}{N} & =\operatorname{Tr} \Omega, \\
\dot{\Omega} & =-i \Omega^{2}+i \varpi^{2},
\end{aligned}
$$

em que:

\footnotetext{
${ }^{1}$ No formalismo funcional de Schrödinger, as integrais funcionais gaussianas serão representadas na forma:

$$
\begin{gathered}
\int \mathcal{D}^{2} \phi\left[-\int d^{3} x d^{3} y \phi^{*} \Omega \phi+\int d^{3} x\left(a^{*} \phi+\phi^{*} b\right)\right]= \\
=\operatorname{det}(\Omega)^{-1} \exp \left[d^{3} x d^{3} y a^{*} \Omega^{-1} b\right] .
\end{gathered}
$$

onde $\operatorname{det}(\Omega)=\exp \operatorname{Tr} \ln \Omega$, em que esse logaritmo é um logaritmo funcional e $\operatorname{Tr} \Omega=\int d^{3} x \Omega(x)$.
} 


$$
\begin{array}{r}
\operatorname{Tr} \Omega=\int d^{3} x \Omega\left(x, x^{\prime}, t\right), \\
\Omega^{2}=\int d^{3} y \Omega(x, y, t) \Omega\left(y, x^{\prime}, t\right), \\
\varpi=-\nabla^{2}+m^{2}+2 i e_{b} A^{i}(\mathbf{x}, t) \partial_{i} .
\end{array}
$$

Temos que lembrar que, em nosso caso, o campo externo dependerá somente do tempo.

Se formos para o espaço dos momentos, considerando esse funcional como uma função de infinitos componentes de Fourier do campo,

$$
\Psi=\prod_{\mathbf{k}} \Psi_{\mathbf{k}}\left(\phi_{\mathbf{k}}^{*} \phi_{\mathbf{k}}\right)
$$

podemos reescrever a equação de Schrödinger (5.102) na forma:

$$
H_{\mathbf{k}} \Psi=-\frac{1}{2} \frac{\partial^{2} \Psi}{\partial \phi_{\mathbf{k}}^{2}}+\frac{1}{2} \omega_{\mathbf{k}}^{2}(t) \phi^{2} \Psi=i \frac{\partial \Psi}{\partial t},
$$

em que a freqüência é a mesma que a dada na seção (5.9). O estado gaussiano é dado por

$$
\Psi_{\mathbf{k}}\left[\phi, \phi^{*}, t\right]=N_{\mathbf{k}}(t) \mathrm{e}^{-\Omega_{\mathbf{k}}(t) \phi_{\mathbf{k}}^{2}}
$$

As equações para as funções $N_{\mathbf{k}}(t)$ e $\Omega_{\mathbf{k}}(t)$ são obtidas inserindo o estado gaussiano em (5.110):

$$
i \frac{\dot{N}_{\mathbf{k}}}{N_{\mathbf{k}}}=\Omega_{\mathbf{k}}, \quad \dot{\Omega}_{\mathbf{k}}=-i \Omega_{\mathbf{k}}^{2}+i \omega_{\mathbf{k}}^{2} .
$$

As equações diferenciais para as quantidades complexas $\Omega(t)$ e $\Omega_{\mathbf{k}}(t)$ são equações de Ricati não lineares. No caso geral, a solução de (5.112), para cada modo, pode ser escrita na forma:

$$
\Omega_{k}=-i \frac{\dot{f_{\mathrm{k}}}}{f_{\mathrm{k}}}
$$

em que $f_{\mathbf{k}}$ obedece à mesma equação que as componentes do campo obedecem:

$$
\ddot{f}_{\mathbf{k}}+\omega_{\mathbf{k}}^{2} f_{\mathbf{k}}=0
$$

As funções $N(t)$ e $N_{\mathbf{k}}(t)$ podem ser obtidas pela normalização do campo, $\langle\Psi \mid \Psi\rangle=1$. Com isso temos que, para cada modo,

$$
N_{\mathbf{k}}(t)=\left[\frac{2(\operatorname{Re} \Omega)}{\pi}\right]^{1 / 2} .
$$


Notamos um fator 1/2, ao invés do convencional $1 / 4$ na normalização. Isto se deve ao fato de estarmos utilizando um campo escalar complexo.

Logo, podemos escrever o estado fundamental do campo na forma:

$$
\Psi=\prod_{k}\left[\frac{2\left(\operatorname{Re} \Omega_{\mathbf{k}}\right)}{\pi}\right]^{1 / 2} \mathrm{e}^{-\Omega_{\mathbf{k}}(t) \phi_{\mathbf{k}}^{2}}
$$

tal que $\Omega_{k}$ obedece (5.113) e (5.114) . Portanto, dada uma solução particular para $\Omega_{k}$, nosso funcional de onda fica totalmente definido.

Com isso, para podermos calcular a probabilidade de não emitir pares, podemos calcular a densidade de probabilidade entre dois estados gaussianos:

$$
\begin{aligned}
\left\langle\Psi_{1} \mid \Psi_{2}\right\rangle & =\int \prod_{k} d \phi_{\mathbf{k}}^{\dagger} d \phi_{\mathbf{k}} \Psi_{1}^{*} \Psi_{2} \\
& =\int \prod_{k} d \phi_{\mathbf{k}}^{\dagger} d \phi_{\mathbf{k}}\left[\frac{2\left(\operatorname{Re} \Omega_{1, \mathbf{k}}\right)}{\pi}\right]^{1 / 2}\left[\frac{2\left(\operatorname{Re} \Omega_{2, \mathbf{k}}\right)}{\pi}\right]^{1 / 2} \mathrm{e}^{-\sum_{k}\left(\Omega_{\mathbf{1}, \mathbf{k}}^{*}(t)+\Omega_{\mathbf{2}, \mathbf{k}}(t)\right) \phi_{\mathbf{k}}^{2}},
\end{aligned}
$$

e portanto, fazendo integrações gaussianas, temos que:

$$
\left|\left\langle\Psi_{1} \mid \Psi_{2}\right\rangle\right|^{2}=\operatorname{det}\left[\frac{4\left(\operatorname{Re} \Omega_{1}\right)\left(\operatorname{Re} \Omega_{2}\right)}{\left(\Omega_{1}^{*}+\Omega_{2}\right)\left(\Omega_{1}+\Omega_{2}^{*}\right)}\right] .
$$

Escrevendo $\Omega$ da forma (5.113), podemos reescrever a equação acima em termo das funções $f$ :

$$
\left|\left\langle\Psi_{1} \mid \Psi_{2}\right\rangle\right|^{2}=\operatorname{det}\left[\frac{\left(\dot{f}_{1, \mathbf{k}} f_{1, \mathbf{k}}^{*}-\dot{f}_{1, \mathbf{k}}^{*} f_{1, \mathbf{k}}\right)\left(\dot{f}_{2, \mathbf{k}} f_{2, \mathbf{k}}^{*}-\dot{f}_{2, \mathbf{k}}^{*} f_{2, \mathbf{k}}\right)}{\left(\dot{f}_{1, \mathbf{k}}^{*} f_{2, \mathbf{k}}-\dot{f}_{2, \mathbf{k}} f_{1, \mathbf{k}}^{*}\right)\left(\dot{f}_{2, \mathbf{k}}^{*} f_{2, \mathbf{k}}-\dot{f}_{1, \mathbf{k}} f_{2, \mathbf{k}}^{*}\right)}\right] .
$$

Supondo agora que $\Psi_{1}$ representa o funcional de um estado inicial (in) e $\Psi_{2}$ de um estado final (out), respectivamente antes e depois do campo externo ter sido ligado, podemos então relacionar essas funções através de uma transformada de Bogoliubov como feito anteriormente, uma vez que o campo externo depende somente do tempo. Assim, temos que:

$$
f_{2, \mathbf{k}}=\alpha_{k} f_{1, \mathbf{k}}+\beta_{\mathbf{k}} f_{1, \mathbf{k}}^{*}
$$

$\mathrm{Ou}$,

$$
f_{1, \mathbf{k}}=\alpha_{k}^{*} f_{2, \mathbf{k}}-\beta_{\mathbf{k}} f_{2, \mathbf{k}}^{*}
$$

em que os coeficientes de Bogoliubov são escolhidos para terem normalização análoga à feita no caso acima: 


$$
\left|\alpha_{n}\right|^{2}-\left|\beta_{n}\right|^{2}=1
$$

Com essa relação, os wronskianos $\left(\dot{f}_{1, \mathbf{k}} f_{1, \mathbf{k}}^{*}-\dot{f}_{1, \mathbf{k}}^{*} f_{1, \mathbf{k}}\right)$ e $\left(\dot{f}_{2, \mathbf{k}} f_{2, \mathbf{k}}^{*}-\dot{f}_{2, \mathbf{k}}^{*} f_{2, \mathbf{k}}\right)$ são constantes e iguais a um. Portanto, temos que a probabilidade de não emitir pares é dada por:

$$
P_{0, b}=\operatorname{det}\left(\frac{1}{\left|\alpha_{k}\right|^{2}}\right)=\operatorname{det}\left(\frac{1}{1+\left|\beta_{k}\right|^{2}}\right)=\operatorname{det}\left(\frac{1}{1+\left\langle N_{k}(t)\right\rangle_{0}}\right) \text {. }
$$

Como $f_{\mathbf{k}}$ tem a mesma evolução que o campo descrita em (5.10) e (5.114), então a evolução de $f_{\mathbf{k}}$ representa a evolução dos modos do campo. Logo, as transformadas de Bogoliubov (5.121) e (5.122) são as mesmas que as feitas para os campos na representação de Heisenberg acima e, com isso, seus coeficientes de Bogoliubov coincidem.

Os valores esperados do operador número e a probabilidade de não emitir pares calculadas acima dependem do cálculo dos coeficientes de Bogoliubov. Esses coeficientes são determinados para cada tipo de campo externo do problema. Logo, as expressões acima são gerais, válidas para quaisquer campos externos.

É importante notar que, nessa descrição, a criação de partículas ocorre devido a um mecanismo de mistura dos modos, devido às transformadas de Bogoliubov.

Vamos determinar os coeficientes de Bogoliubov para um campo elétrico estático, com potencial externo igual ao usado na seções anteriores, $\mathbf{A}=(0,0,0, A(t))=(0,0,0,-E t)$. A analogia para um gauge em que $A=A(x)$ pode ser vista na referência [110].

\subsubsection{Determinação dos coeficientes de Bogoliubov: campo escalar}

Para determinarmos os coeficientes de Bogoliubov, vamos reescrever a equação (5.10) na forma:

$$
\frac{d^{2} \phi_{\mathbf{k}}}{d \tau^{2}}+\underbrace{\left(\Upsilon+\tau^{2}\right)}_{\omega^{2}(\tau)} \phi_{\mathbf{k}}=0,
$$

em que $\tau \equiv \frac{\sqrt{e_{b} E t}-k_{z}}{\sqrt{e_{b} E}}$ e $\Upsilon \equiv \frac{\left(k_{\perp}^{2}+m^{2}\right)}{e_{b} E}$. Essa equação pode ser integrada e sua solução é dada pelas funções parabólicas cilíndricas $D_{p}(z)$ :

$$
\phi_{\mathbf{k}}(\tau)=\lambda_{\mathbf{k}} D_{-(1+i \Upsilon) / 2}[(1+i) \tau]+\gamma_{\mathbf{k}} D_{-(1+i \Upsilon) / 2}[-(1+i) \tau]
$$

As constantes $\lambda_{\mathbf{k}}$ e $\gamma_{\mathbf{k}}$ são fixadas pelas condições iniciais.

Queremos escolher nosso estado inicial como sendo vácuo para valores de $\tau$ muito negativos. Para isso temos que definir um estado adiabático em relação à freqüência $\omega(\tau)$, como estado inicial. A aproximação WKB, ou aproximação adiabática, é a ferramenta que utilizamos para isso. Com essa aproximação podemos também aproximar a forma da 
solução.

A aproximação WKB é válida no limite em que:

$$
\left|Q / \omega^{2}\right| \ll 1
$$

onde $Q$ é definido como sendo:

$$
Q \equiv \frac{3}{4} \frac{1}{\omega^{2}}\left(\frac{d \omega}{d \tau}\right)^{2}-\frac{1}{2 \omega} \frac{d^{2} \omega}{d \tau^{2}}
$$

e vem da equação de movimento:

$$
\ddot{\phi}_{\mathbf{k}}+\left[\omega^{2}(\tau)-Q\right] \phi_{\mathbf{k}}=0 .
$$

A função de modo WKB, solução de (5.129), é dada por $\phi_{w k b, \mathbf{k}}=\frac{1}{\sqrt{2 \omega}} \exp \left( \pm \int \omega d \tau\right)$.

Em nosso caso (5.125), a condição WKB, ou condição de adiabaticidade, é dada por:

$$
\left|\frac{Q}{\omega^{2}}\right|=\frac{1}{\Upsilon^{2}} \frac{1}{2\left(1+\frac{\tau^{2}}{\Upsilon}\right)^{2}}\left|\frac{5 \frac{\tau^{2}}{\Upsilon}}{2\left(1+\frac{\tau^{2}}{\Upsilon}\right)}-1\right|,
$$

e, nos instantes em que essa condição é válida, podemos determinar a solução aproximada:

$$
\begin{aligned}
\phi_{\mathbf{k}}(\tau) & \simeq \alpha_{k} \phi_{w k b, \mathbf{k}}(\tau)+\beta_{k} \phi_{w k b, \mathbf{k}}^{*}(\tau) \\
& =\frac{\alpha_{k}}{\sqrt{2 \omega}} \exp \left(-i \int_{\tau_{i n i}}^{\tau} \omega\left(\tau^{\prime}\right) d \tau^{\prime}\right)+\frac{\beta_{k}}{\sqrt{2 \omega}} \exp \left(+i \int_{\tau_{i n i}}^{\tau} \omega\left(\tau^{\prime}\right) d \tau^{\prime}\right),
\end{aligned}
$$

em que $\tau_{\text {ini }}<0$.

Podemos verificar que no limite $\tau / \sqrt{\Upsilon} \rightarrow \pm \infty,\left|Q / \omega^{2}\right|$ é igual a zero e a aproximação WKB é válida. Com isso, podemos definir bem o vácuo nessas regiões, $|0\rangle_{\text {in }}$ em $\tau / \sqrt{\Upsilon} \rightarrow$ $-\infty$ e $|0\rangle_{\text {out }}$ em $\tau / \sqrt{\Upsilon} \rightarrow+\infty$. Assim, é possível determinar o estado inicial como estando em vácuo adiabático $|0\rangle_{i n}$ em $\tau / \sqrt{\Upsilon} \rightarrow-\infty$. Nesse limite, $\alpha_{k}=1$ e $\beta_{k}=0$, e, portanto, $\lim _{\tau / \sqrt{\Upsilon} \rightarrow-\infty} \phi_{\mathbf{k}}=\phi_{w k b, \mathbf{k}}$. Esse critério especifica completamente os coeficientes $\lambda_{\mathbf{k}}$ e $\gamma_{\mathbf{k}}$ e conseqüentemente determina os coeficientes de Bogoliubov na região out.

Podemos ver em (5.130) que entre esses estados adiabáticos in e out, em torno de $\tau=0$, temos a violação da adiabaticidade. Nesse instante ocorre a criação de partículas.

Para determinarmos os coeficientes de Bogoliubov, podemos ver que a fase $\int_{\tau_{i n i}}^{\tau} \omega\left(\tau^{\prime}\right) d \tau^{\prime}$, com $\omega(\tau)$ dado acima, pode ser escrita como: 


$$
\begin{aligned}
\int_{\tau_{i n i}}^{\tau} \omega\left(\tau^{\prime}\right) d \tau^{\prime} & =\frac{\Upsilon}{2}\left[\frac{\tau}{\sqrt{\Upsilon}} \sqrt{1+\frac{\tau^{2}}{\Upsilon}}-\frac{\tau_{i n i}}{\sqrt{\Upsilon}} \sqrt{1+\frac{\tau_{i n i}^{2}}{\Upsilon}}\right] \\
& +\ln \left(\frac{\tau}{\sqrt{\Upsilon}}+\sqrt{1+\frac{\tau^{2}}{\Upsilon}}\right)-\ln \left(\frac{\tau_{i n i}}{\sqrt{\Upsilon}}+\sqrt{1+\frac{\tau_{i n i}^{2}}{\Upsilon}}\right)
\end{aligned}
$$

que no limite $\frac{\tau}{\sqrt{\Upsilon}} \gg 1$, tende a:

$$
\int_{\tau_{i n i}}^{\tau} \omega\left(\tau^{\prime}\right) d \tau^{\prime} \rightarrow \frac{1}{2}\left(\tau|\tau|+\left|\tau_{i n i}\right|^{2}\right)+\frac{\Upsilon}{2}\left(\frac{|\tau|}{\tau} \ln |\tau|+\ln \left|\tau_{i n i}\right|\right) .
$$

Portanto, a função WKB fica na forma:

$$
\phi_{w k b, \mathbf{k}}=\frac{1}{\sqrt{2}} \mathrm{e}^{-i\left(\tau|\tau|+\left|\tau_{i n i}\right|^{2}\right) / 2}|\tau|^{-1 / 2-i|\tau| \Upsilon /(2 \tau)}\left|\tau_{i n i}\right|^{-i \Upsilon / 2} .
$$

No limite em que $\tau \rightarrow-\infty$ a solução exata dada por (5.126), pode ser escrita como:

$$
\begin{aligned}
\phi_{\mathbf{k}}= & \underbrace{\lambda_{\mathbf{k}} \frac{\sqrt{2 \pi}}{\Gamma\left(\frac{1}{2}+\frac{i \Upsilon}{2}\right)} \mathrm{e}^{-i \pi(1-i \Upsilon) / 4}(1-i)^{-(1+i \Upsilon) / 2} \sqrt{2} \mathrm{e}^{i\left|\tau_{i n i}\right|^{2} / 2}\left|\tau_{i n i}\right|^{i \Upsilon / 2}}_{\alpha_{k}} \phi_{w k b, \mathbf{k}}+ \\
& +\underbrace{\left[\lambda_{\mathbf{k}} \mathrm{e}^{i \pi(1+i \Upsilon) / 2}+\gamma_{\mathbf{k}}\right](1+i)^{-(1+i \Upsilon) / 2} \sqrt{2} \mathrm{e}^{-i\left|\tau_{i n i}\right|^{2} / 2}\left|\tau_{i n i}\right|^{-i \Upsilon / 2}}_{\beta_{k}} \phi_{w k b, \mathbf{k}}^{*},
\end{aligned}
$$

e como $\alpha_{k}=1$ e $\beta_{k}=0$ nesse limite, podemos determinar os coeficientes $\lambda_{\mathbf{k}}$ e $\gamma_{\mathbf{k}}$ :

$$
\begin{aligned}
& \lambda_{\mathbf{k}}=\frac{\Gamma\left(\frac{1}{2}+\frac{i \Upsilon}{2}\right)}{\sqrt{2 \pi}} \mathrm{e}^{i \pi(1-i \Upsilon) / 4}(1-i)^{(1+i \Upsilon) / 2} \frac{1}{\sqrt{2}} \mathrm{e}^{-i\left|\tau_{i n i}\right|^{2} / 2}\left|\tau_{i n i}\right|^{-i \Upsilon / 2} \\
& \gamma_{\mathbf{k}}=-\lambda_{\mathbf{k}} \mathrm{e}^{i \pi(1+i \Upsilon) / 2}
\end{aligned}
$$

Portanto, considerando o comportamento no limite em que $\tau \rightarrow+\infty$ e, usando os resultados acima, podemos determinar os coeficientes de Bogoliubov:

$$
\left|\alpha_{k}\right|^{2}=1+\mathrm{e}^{-\pi \Upsilon}, \quad\left|\beta_{k}\right|^{2}=\mathrm{e}^{-\pi \Upsilon}
$$

que satisfaz a condição de Bogoliubov (5.80).

Determinados os coeficientes de Bogoliubov podemos finalmente calcular $\langle N(t)\rangle$ e $P_{0 b}$. A probabilidade de não emitir pares, dada pela equação (5.124), com os coeficientes obtidos acima, é dada por: 


$$
P_{0 b}=\operatorname{det}\left(\frac{1}{1+\mathrm{e}^{-\pi \Upsilon}}\right)=\exp \left[-\operatorname{Tr} \ln \left(1+\mathrm{e}^{-\pi \Upsilon}\right)\right]
$$

Lembrando que o traço é dado por $\operatorname{Tr} \rightarrow \int d^{3} x$, podemos fazer o cálculo acima e obtemos:

$$
\begin{aligned}
P_{0 b} & =\exp \left[-V T \frac{\alpha_{b} E^{2}}{2 \pi^{2}} \sum_{n=1}^{\infty} \frac{(-1)^{n+1}}{n^{2}} \exp \left(\frac{-n \pi m_{b}^{2}}{e_{b} E}\right)\right] \\
& =\exp \left[-V T \omega_{b}\right] .
\end{aligned}
$$

Esse resultado é o mesmo que o apresentado por Schwinger em seu artigo original e equivale ao obtido via teoria de espalhamento, apresentado aqui.

Para calcularmos o número de partículas criadas temos, de (5.94 ), que:

$$
\left\langle N_{k}(t)\right\rangle_{0}=\mathrm{e}^{-\pi \Upsilon}=\exp \left[-\frac{\pi\left(\mathbf{k}^{\perp}+m^{2}\right)}{e E}\right] .
$$

O mesmo número de antipartículas é criado.

\subsubsection{Formalismo dos estados squeezed}

Vamos expor o efeito Schwinger no formalismo dos estados squeezed com o intuito de termos uma visão gráfica da criação de partículas. Nesse formalismo iremos apenas reexpressar as transformações de Bogoliubov de uma maneira diferente. De forma ilustrativa vamos somente expressar esse resultado para o campo escalar.

Partindo de (5.90), podemos reparametrizar esses coeficientes de Bogoliubov da forma:

$$
\begin{aligned}
& x_{1 k}=e^{-i\left(\theta_{k}-2 \phi_{k}\right)} \sinh r_{k}, \\
& x_{2 k}=e^{i \theta_{k}} \cosh r_{k},
\end{aligned}
$$

em que as quantidades $r_{k}, \theta_{k}$ e $\phi_{k}$ são funções do tempo e $r_{k}(0)=\theta_{k}(0)=0$. Essas equações obedecem equações equivalentes à (5.93):

$$
\begin{aligned}
2 \dot{r}_{k} & =\frac{\dot{\omega}_{k}}{\omega_{k}} \cos 2 \phi_{k}, \\
2 \dot{\phi}_{k} & =-2 \omega_{k}-\frac{\dot{\omega}_{k}}{\omega_{k}} \operatorname{sen} 2 \phi_{k} \operatorname{coth} 2 r_{k}, \\
\dot{\theta} & =-\omega_{k}-\frac{1}{2} \frac{\dot{\omega}_{k}}{\omega_{k}} \operatorname{sen} 2 \phi_{k} \tanh 2 r_{k} .
\end{aligned}
$$


Com os coeficientes determinados por essas equações, podemos reescrever o número de partículas criadas por meio da equação (5.94) para criação espontânea, em termo do parâmetro de squeezing:

$$
\left\langle N_{\mathbf{k}}(t)\right\rangle=\cosh ^{2} r_{k}
$$

Com isso, podemos descrever a evolução temporal do número de partículas criadas ${ }^{2}$. Isso pode ser visto na Figura 5.1, em que no mesmo gráfico incluímos a parâmetro WKB. Podemos ver que a criação de partículas ocorre nos instantes em que há violação WKB, que acontece entre os instantes inicial e final em que não há criação, representados pelos platôs.

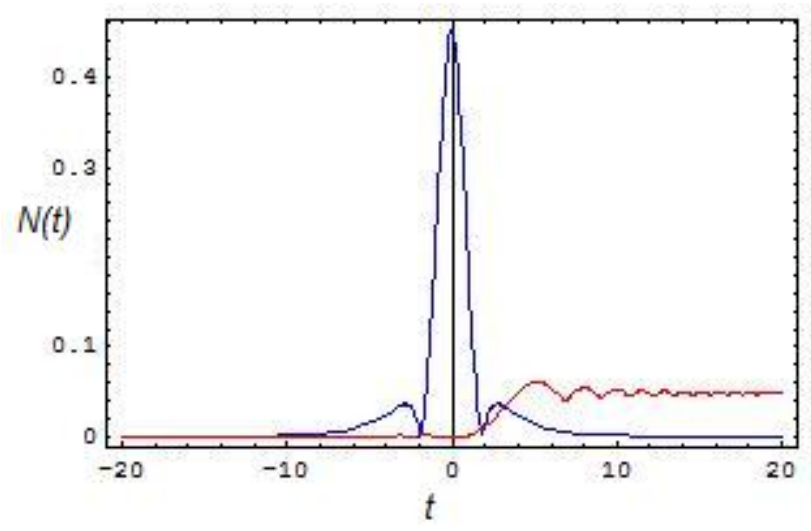

Figura 5.1: O gráfico mostra a densidade de número de partículas criadas em função do tempo (em vermelho). No mesmo gráfico mostramos o parâmetro WKB, em que podemos ver que a criação de partículas ocorre exatamente nos instantes em que há a violação (em azul). A simulação foi feita para valores arbitrários dos parâmetros $k\left(k_{\perp}=0,1 \mathrm{e}\right.$ $\left.k_{z}=0,01\right)$ e $m^{2}=0,2$.

\footnotetext{
${ }^{2} \mathrm{Na}$ verdade esse é a densidade de número de partículas. Para seu valor total é preciso integrar essa quantidade como mostrado em (5.96).
} 


\section{Capítulo 6}

\section{Efeito Schwinger Dinâmico}

Neste capítulo vamos estudar o análogo dinâmico do efeito Schwinger, em que a criação de partículas ocorre devido à aplicação de um campo variável com o tempo, em lugar de um campo estático. O surgimento desse efeito deu-se uma vez que, para o efeito Schwinger estático ocorrer, verificamos que seriam necessários campos elétricos estáticos muito fortes, da ordem de $E_{c}=\frac{m^{2} c^{3}}{e \hbar} \approx 1,4 \times 10^{16} \mathrm{~V} / \mathrm{cm}$. Campos assim são muito difíceis de serem criados, dado que mesmo a tecnologia atual de lasers ainda está algumas ordens de magnitude abaixo desse valor, e a criação de campos elétricos estáticos é um desafio ainda maior.

Portanto, é interessante generalizar esse efeito. A generalização natural do efeito Schwinger estático é o efeito Schwinger dinâmico (ESD), no qual o campo externo aplicado varia com o tempo.

O problema da produção de pares por um campo externo inomogêneo já havia sido apontado por Schwinger em 1954 [120] . Contudo, esse efeito somente foi estudado a partir dos anos 1970. Quando se começou a pensar nesse problema, logo se percebeu que a forma do potencial utilizado influenciaria muito no resultado, uma vez que não é possível resolver o problema para qualquer tipo de potencial. Havia o interesse claro de computar a criação de partículas no caso de lasers para que o efeito fosse descrito num contexto mais próximo da física experimental. No caso de lasers, a forma do campo elétrico externo é senoidal, $E(t)=E_{0}$ sen $\left(\omega_{0} t\right)$, ou cossenoidal, em que $\omega_{0}$ é a freqüência do campo externo alternante. Contudo, para esse campo não é possível resolver analiticamente a Lagrangeana efetiva, computando-a em uma forma fechada, como no caso da Lagrangeana efetiva de Euler-Heisenberg. Então utiliza-se uma aproximação semi-clássica para calcular o efeito. Essa aproximação foi estudada detalhadamente por Brezin e Itzykson [74], baseado no trabalho de L. V. Keldysh [73], e por Popov e Marinov [121]. Nesse caso, o efeito Schwinger dinâmico é chamado de efeito Schwinger oscilatório (ESO). Esse será nosso caso de estudo. Nosso interesse aparece dada sua analogia com o caso gravitacional (veja o capítulo 7), utilizando campos externos também oscilatórios, mas de origem gravitacional. Esse caso também é importante já que, com a recente melhoria na 
tecnologia de lasers, principalmente com o experimento XFEL [122], uma possibilidade de detecção do efeito Schwinger oscilatório surge novamente. Com isso, poderíamos ter a comprovação dos mecanismos não-perturbativos de criação de partículas por campos fortes e, conseqüentemente, a validação das analogias com sistemas em campos gravitacionais externos.

Existe um número limitado de casos de potenciais em que a Lagrangeana efetiva tem forma fechada, ou até mesmo que o efeito Schwinger dinâmico pode ser calculado por outras maneiras, por exemplo via transformadas de Bogoliubov, sem que seja necessária a utilização de aproximações. O caso mais conhecido e estudado é o do chamado potencial de Sauter, em homenagem a F. Sauter, em que $E(t)=E_{0} / \cosh ^{2}\left(k_{0} t\right) \Rightarrow A(t)=$ $\left(\frac{E_{0}}{k_{0}}\right) \tanh \left(k_{0} t\right)$. Narozhnyi e Nikishov [123] foram os principais autores que estudaram esse efeito utilizando a descrição via transformadas de Bogoliubov. Eles verificaram que, para um campo externo desse tipo, as equações de Dirac e Klein-Gordon se reduzem a equações hipergeométricas e podem ser facilmente resolvidas.

Finalmente, alguns anos depois, Popov [153], via cálculo da Lagrangeana efetiva, derivou a probabilidade de produção de pares exata para um campo elétrico com dependência temporal arbitrária. Para esse campo verificou-se que o problema se reduz a encontrar o comportamento assintótico da solução de uma equação clássica simples do tipo oscilador harmônico.

Posteriormente, uma nova classe de fenômenos de criação de partículas dinâmica (ou criação de partículas por sistemas quânticos com parâmetros dependentes do tempo) começou a surgir. Esses fenômenos surgiram também como generalizações de um outro fenômeno de instabilidade do vácuo quântico: o efeito Casimir. O efeito Casimir [96, 97, 98] consiste, simplificadamente, no surgimento de uma força entre placas paralelas no vácuo, devido às flutuações do vácuo. O efeito Casimir dinâmico (ECD) é o fenômeno de criação de fótons a partir do vácuo, quando essas placas movem-se aceleradamente. Ele se assemelha ao efeito Schwinger dinâmico, uma vez que fronteiras móveis, ou as condições de contorno de fronteiras móveis, são equivalentes a potenciais dependentes do tempo. Isso foi sugerido por Munier et al [125], em 1980, em um problema de uma partícula livre de massa $m$ presa dentro de uma caixa unidimensional com paredes impenetráveis, em que uma dessas paredes estava em movimento. Nesse trabalho, os autores resolveram o problema sujeito a condições de contorno da parede móvel. Contudo, fazendo transformações canônicas apropriadas, verificaram que, na equação de Schrödinger do problema, surgia um termo dependente do tempo no lado direito da equação. Isso corresponde exatamente ao problema de um campo livre em presença de um campo externo dependente do tempo. Assim, surgiu a analogia entre o que seria chamado de efeito Casimir dinâmico e o efeito Schwinger dinâmico. Isso nos leva a concluir que a analogia entre problemas em um campo externo aplicado com métricas de fundo (como métricas não estáticas ), assim como com paredes móveis, também pode ser feita para o fenômeno de criação de 
partículas. Veremos essa questão com detalhe no Capítulo 7.

A derivação completa do efeito Casimir dinâmico foi feita a partir desse trabalho de Munier. Principalmente após o estudo desse efeito nos anos 90, por Schwinger [126], que foi quem nomeou o efeito, é que os cálculos do ECD começaram a surgir, principalmente utilizando as transformações de Bogoliubov . O aspecto mais interessante do efeito Casimir dinâmico é que a verificação experimental começa a se tornar possível atualmente, com um experimento idealizado por um grupo de cientistas italianos em fase final de preparação: o experimento MIR (Motion Induced Radiation - Movimento Induzido pela Radiação) [127]. Devido à analogia entre os ECD e o ESD, e, uma vez que o ESD ainda tem que esperar pela próxima geração de lasers, o ECD surge como a grande esperança de verificação experimental da criação de partículas dinâmica.

Neste capítulo iremos calcular o efeito Schwinger oscilatório, em que o campo externo aplicado tem um comportamento oscilatório. Descreveremos esse efeito em dois formalismos: via teoria de espalhamento, baseado no trabalho de Itzykson e Brezin [74], e via transformações de Bogoliubov. A descrição via teoria de espalhamento é feita nesse capítulo de forma completa, seguindo um método derivado do método Schwinger. Apesar de ser muito extensa, sua principal motivação pode ser vista no final do cálculo, em que os limites em que a criação de partículas ocorre são determinados. Em seguida, investigaremos o caso central do capítulo e que deve ser considerado com mais atenção, em que a criação de partículas é determinada via transformadas de Bogoliubov. Como mencionamos, para o potencial que iremos utilizar não temos um resultado analítico para descrevermos o problema via essas transformadas. Com isso, desenvolvemos nesse trabalho um método analítico baseado no método utilizado para o pré-aquecimento [46], para o cálculo via transformadas de Bogoliubov do efeito Schwinger oscilatório. Esse método ainda não foi aplicado na literatura no caso do efeito Schwinger oscilatório e fornece uma maneira muito mais clara e direta de determinação desse efeito. A importância desse formalismo reside no fato de que é a partir dessa descrição que faremos a analogia com a criação de partículas no pré-aquecimento no Capítulo 7. Apresentamos, como uma extensão do formalismo via transformada de Bogoliubov, a descrição da criação de partículas via formalismo dos estados squeezed, utilizado para a obtenção da evolução do número de partículas criadas. O mecanismo de criação de partícula que está presente em cada formalismo também será discutido.

Em seguida, passaremos para a descrição do ECD via formalismo de Bogoliubov. Faremos a exposição do fenômeno de maneira simplificada em $1+1$ dimensões (uma dimensão espacial e uma temporal), utilizando condições de contorno de Dirichlet nas fronteiras e em $3+1$ dimensões determinando o número de partículas criadas no regime de ressonância paramétrica para o modo TM. Estabelecido o formalismo do efeito, descreveremos o experimento que é o mais forte candidato para verificação do ECD, e com isso, da criação de partículas dinâmica: o experimento MIR do grupo de Pádua, na Itália. Dessa 
descrição extensa e detalhada, temos como principais componentes que devemos nos ater a analogia com a criação de partículas no efeito Schiwnger oscilatório e a determinação do número de partículas criadas, importante para o entendimento da possibilidade de verificação experimental desse efeito pelo experimento MIR.

\subsection{Efeito Schwinger Oscilatório via teoria de espalha- mento}

Como uma generalização da descrição feita no capítulo anterior para o efeito Schwinger estático via teoria de espalhamento, descreveremos agora, pelo mesmo método, o ESO. Trataremos aqui do caso de criação de partículas do vácuo por um campo elétrico oscilatório. Essa descrição é importante, uma vez que, como vimos, os limites observacionais do ESE ainda são inacessíveis. Assim, é interessante estudar como um campo oscilatório pode modificar esses limites, verificando a dependência da criação de partículas com freqüência do campo externo aplicado. É possível, dessa forma, fazer estimativas práticas das ordens de magnitude da taxa de criação de partículas para um campo oscilatório e das condições em que se dá essa criação.

Para obtermos essas estimativas, é natural realizarmos os cálculos para a criação de elétrons e pósitrons, ou seja, utilizando o campo fermiônico como o campo quântico das partículas. Contudo, como vimos na Seção 5.2.1, os spins dos pares criados contribuem essencialmente com um fator multiplicativo em relação aos cálculos feitos para bósons carregados. Com isso faremos nossos cálculos para o campo escalar (bosônico) complexo.

Vamos tratar então o problema de criação de partículas como um problema de espalhamento. Essa descrição, baseada em [74], é uma aproximação, então é feita de forma bastante detalhada aqui, para que esteja bem determinada. Sua descrição é uma demostração de um dos métodos de obtenção do efeito Schwinger oscilatório. Contudo, seu principal resultado que queremos obter e que será utilizado na dissertação são seus limites de observação.

Impomos que o campo externo aplicado é um campo puramente elétrico oscilatório com dependência apenas temporal, o que nos permite obter as características principais do fenômeno sem perda de generalidade. Exigimos também que esse campo seja igual a zero para tempos grandes, ou seja, que ele seja adiabaticamente ligado e desligado em um tempo finito. Com isso, é possível associar o problema com um espalhamento de uma onda livre em um potencial oscilatório, descrito por uma equação de Lippmann- Schwinger unidimensional. Com isso em mãos, será possível utilizar o método semiclássico, ou WKB, para obtermos as soluções do problema.

Após estabelecido o problema, obteremos a taxa de criação de partículas utilizando os resultados obtidos na Seção 5.2.1 para campos arbitrários. 
Para podermos descrever o problema de um campo escalar complexo em um potencial oscilatório será necessário fazermos algumas simplificações. Limitamos as freqüência do campo externo variável a $\omega_{0} \ll m$. Além disso, assumimos que $e E \ll m^{2}$. Quando formos calcular a taxa de criação de partículas, por meio de (5.58), veremos que essa condição nos diz que os termos que correspondem à criação de mais de um par podem ser ignorados. Essa limitação é satisfeita por todos os feixes de lasers existentes. É interessante perceber que, no cálculo oscilatório, diferentemente do estático, a própria condição do problema já nos fornece condições de energia para que o efeito seja energeticamente possível de ser observado.

Vamos considerar o sistema de um campo escalar complexo interagindo com um potencial oscilatório, dependente do tempo com freqüência $\omega_{0}, A(x)=(0,0,0, A(t))$. Esse sistema é descrito pela equação de Klein-Gordon:

$$
\left[\frac{d}{d t}+\omega_{\mathbf{k}}^{2}(t)\right] \phi_{\mathbf{k}}(t)=0
$$

com freqüência $\omega_{\mathbf{k}}^{2}(t)=k_{\perp}^{2}+m^{2}+\left(k_{3}-e A(t)\right)^{2}$. Podemos interpretar essa equação de uma outra maneira.

Na Seção 5.2.1, calculamos a forma da taxa de criação de partículas para campos arbitrários no caso escalar carregado dada por (5.58):

$$
\omega_{B}(x)=\left\langle x\left|\ln \left[I-\mathcal{T}(\mathcal{A}) \rho^{(+)} \overline{\mathcal{T}}(A) \rho^{(-)}\right]\right| x\right\rangle,
$$

em que os operadores de transição $\mathcal{T}(\mathcal{A})$ e $\overline{\mathcal{T}}(A)$ são dados por $(5.50)$ e (5.51), respectivamente, e $V=-2 k_{3} A(t)+e^{2} A^{2}(t)$.

Definidos esses operadores e o potencial, nosso problema reduz-se a um problema de espalhamento por um potencial $V$, descrito pela equação de Lippmann-Schwinger unidimensional [107]:

$$
\left[\frac{d^{2}}{d t^{2}}+\omega^{2}\right] \phi(t)=V(t) \phi(t) .
$$

Essa analogia pode ser feita, uma vez que por hipótese, o potencial é amortecido adiabaticamente para tempos grandes e, assim, longe do potencial, em $|t| \rightarrow \infty$, os estados são livres (ondas planas). Logo, o potencial $V$ pode ser visto como um potencial de curto alcance. Isso estabelece o problema como um espalhamento descrito pela equação de Lippmann-Schwinger. Contudo, essa equação descreve um espalhamento diferente do usual, uma vez que o papel da variável de configuração é feito pelo tempo.

Com esse tratamento dado ao problema, podemos escrever a taxa de criação de partículas em uma forma que pode ser melhor interpretada por essa nova descrição em função da equação de Lippmann-Schwinger. Sabendo que podemos escrever a probabilidade como $P_{0}=e^{W}=e^{\int d^{4} x \omega_{B}}$, então temos que: 


$$
\frac{d W}{d V}=\int d t \int d^{4} k d^{4} k^{\prime}\left\langle k^{\prime}\left|\left[\mathcal{T}(\mathcal{A}) \rho^{(+)} \overline{\mathcal{T}}(A) \rho^{(-)}\right]\right| k\right\rangle e^{-i\left(p-p^{\prime}\right) x},
$$

em que tomamos o traço em (6.2). Logo, calculando explicitamente esse elemento de matriz, temos que a probabilidade por unidade de volume de não emitir pares é dada por:

$$
\frac{d W}{d V}=\frac{1}{2 \pi} \int \frac{d^{3} p}{(2 \omega)^{2}}|\langle-\omega|\mathcal{T}| \omega\rangle|^{2},
$$

com $\omega=\sqrt{\mathbf{p}^{2}+m^{2}}$ e onde $|\omega\rangle$ representa o estado do momento $k_{0}$. O elemento de matriz $|\langle-\omega|\mathcal{T}| \omega\rangle|$ é conhecido, em problemas de espalhamento, como a amplitude de espalhamento para trás, em que o sinal menos no bra significa que o estado final está na direção contrária da inicial. Em nosso caso, essa amplitude é interpretada como a amplitude de probabilidade de criação de partículas.

Para determinarmos a criação de partículas, então, temos que encontrar a amplitude de probabilidade de espalhamento para trás. Para isso, temos que descobrir como essa amplitude é descrita em termos dos parâmetros do espalhamento. Assim, é necessário analisarmos nossa equação de Lippmann-Schwinger e suas soluções.

Como o potencial é, por hipótese, de curto alcance e a variável de configuração é o tempo, podemos obter as soluções da equação de Lippmann-Schwinger (6.3), que se comportam assintoticamente como:

$$
\phi(t) \sim\left\{\begin{array}{l}
e^{-i \omega t}+b e^{i \omega t}, t \rightarrow-\infty \\
a e^{-i \omega t}, t \rightarrow+\infty
\end{array}\right.
$$

Por outro lado, podemos estudar as soluções da equação de Klein-Gordon inomogênea acima, utilizando as funções de Green. A solução é dada por:

$$
\phi(t)=\phi_{0}(t)-\int d t^{\prime} G\left(t-t^{\prime}\right) V\left(t^{\prime}\right) \psi\left(t^{\prime}\right) .
$$

Então, fazendo uma transformada de Fourier, podemos reescrever essa solução da forma:

$$
\begin{aligned}
\phi(t) & =e^{-i \omega t}+\frac{1}{i \omega} \int d t^{\prime} e^{-i \omega\left(t-t^{\prime}\right)} V\left(t^{\prime}\right) \psi\left(t^{\prime}\right) \\
& =e^{-i \omega t}+\frac{f^{\mp}}{\omega} e^{+i \omega t},
\end{aligned}
$$

em que $f^{ \pm}=\langle\mp \omega|\mathcal{T}| \omega\rangle$. Com isso, comparando com a solução assintótica em (6.6), podemos ver que: 


$$
b=\frac{\pi}{i \omega}\langle-\omega|\mathcal{T}| \omega\rangle, \quad(a-1)=\frac{\pi}{i \omega}\langle\omega|\mathcal{T}| \omega\rangle
$$

Identificados os parâmetros da equação de Lippmann-Schwinger com a amplitude de espalhamento, podemos escrever a probabilidade de criação de partículas por unidade de volume na forma:

$$
\frac{d W}{d V}=\frac{1}{(2 \pi)^{3}} \int d^{3} k|b|^{2} .
$$

Logo, precisamos identificar o coeficiente $b$.

Queremos escolher nosso estado inicial como sendo um estado livre, para $t \rightarrow-\infty$. A aproximação WKB, ou aproximação adiabática, é a ferramenta que utilizamos para isso. Com essa aproximação podemos também aproximar a forma da solução.

Se calcularmos a razão:

$$
\frac{\dot{\omega}_{\mathbf{k}}}{\omega_{\mathbf{k}}^{2}}=\frac{e \dot{A}(t)\left(k_{3}-e A(t)\right)}{\left[m^{2}+k_{\perp}^{2}+\left(k_{3}-e A(t)\right)^{2}\right]^{3 / 2}},
$$

que representa o parâmetro WKB, em primeira ordem, podemos ver que para os estados assintóticos (em $|t| \rightarrow \infty$ ), em que o campo externo é nulo, podemos ver que, sendo $E_{0}$ é a amplitude do campo oscilatório:

$$
\left|\frac{\dot{\omega}_{\mathbf{k}}}{\omega_{\mathbf{k}}^{2}}\right|<\frac{e E_{0}}{m^{2}+k_{\perp}^{2}}<\frac{e E_{0}}{m^{2}} \ll 1,
$$

em que utilizamos a condição assumida inicialmente que $e E_{0} \ll m^{2}$. Assim, como a condição WKB é satisfeita em $|t| \rightarrow \infty$, vemos que esses estados são adiabáticos.

Como feito na Seção 5.2.2, nos instantes em que (6.12) é válida, podemos escrever a solução geral da equação de Klein-Gordon na forma:

$$
\begin{aligned}
\phi(t) & =\alpha(t) e^{-i \chi(t)}+\beta(t) e^{i \chi(t)} \\
& =\alpha(t) \phi_{w k b}+\beta(t) \phi_{w k b}^{*},
\end{aligned}
$$

em que $\phi_{w k b}$ são as soluções WKB no instante inicial e $\chi(t)=\int_{0}^{t} d t^{\prime} \omega\left(t^{\prime}\right)$. Nessa solução vemos que, diferentemente do que foi feito na Seção 5.2.2, os coeficientes $\alpha$ e $\beta$, que são os coeficientes de Bogoliubov, englobam a normalização das funções WKB.

Comparando essa solução com o comportamento assintótico obtido em (6.6), podemos ver que a solução acima deve obedecer a:

$$
\alpha(-\infty)=1, \beta(+\infty)=0
$$


que são as condições de contorno do problema. Logo, substituindo essas condições de contorno na solução geral e relacionando as soluções assintóticas (6.6) com o comportamento assintótico de (6.13), podemos determinar os parâmetros $a$ e $b$ em função dos coeficientes de Bogoliubov:

$$
\left\{\begin{array}{l}
a=\alpha(+\infty) \exp \left\{-i \int_{-\infty}^{+\infty} d t[\omega(t)-\omega]\right\} \\
b=\beta(-\infty) \exp \left\{-2 i \int_{-\infty}^{0} d t[\omega(t)-\omega]\right\}
\end{array}\right.
$$

Com isso, podemos reescrever a probabilidade em termo dos coeficientes de Bogoliubov:

$$
\frac{d W}{d V}=\frac{1}{(2 \pi)^{3}} \int d^{3} k|\beta(-\infty)|^{2}
$$

Precisamos então determinar esses coeficientes.

Podemos ver que em (6.13) substituímos a função desconhecida $\phi(t)$ por duas funções desconhecidas, $\alpha(t)$ e $\beta(t)$. Esse procedimento somente é útil desde que tenhamos como impor mais condições sobre esses coeficientes, de tal forma que a determinação de um coeficiente seja suficiente para determinarmos ambos. Assim, vamos impor condições em $\dot{\psi}(t)$ também. Sabemos, da solução assintótica para $t \rightarrow \infty$ em (6.6) , que sua derivada será um número imaginário. Assim, derivando a solução geral podemos dividí-la em uma parte real e uma imaginária e sabemos que a parte real dessa derivada tem que ser igual a zero. Com isso, temos a condição:

$$
\dot{\alpha} e^{-i \chi}+\dot{\beta} e^{i \chi}=0
$$

Por outro lado, se reescrevermos a equação de Klein-Gordon (6.3) substituindo a solução geral, temos que :

$$
\dot{\alpha} e^{-i \chi}-\dot{\beta} e^{i \chi}=-\left[\frac{\dot{\omega}(t)}{\omega(t)}\right]\left(\alpha e^{-i \chi}-\beta e^{i \chi}\right) .
$$

Temos, então, um sistema formado pela equação de Klein-Gordon reescrita acima e pela condição (6.17), que pode ser resolvido fornecendo:

$$
\left\{\begin{array}{l}
\dot{\alpha}=-\left[\frac{\dot{\omega}(t)}{2 \omega(t)}\right]\left(\alpha-\beta e^{2 i \chi}\right) \\
\dot{\beta}=-\left[\frac{\dot{\omega}(t)}{2 \omega(t)}\right]\left(\beta-\alpha e^{2 i \chi}\right) .
\end{array}\right.
$$

Essas equações obedecem a algumas condições. Para obtê-las temos que lembrar que, como sabemos que o campo externo é adiabaticamente ligado e desligado em tempos finitos, então, quando $|t| \rightarrow \infty \Rightarrow \dot{\omega}(t) \rightarrow 0$. Com isso, nesse limite, $\dot{\alpha}, \dot{\beta} \rightarrow 0 \Rightarrow$ $\alpha, \beta \rightarrow$ constante. Contudo, de acordo com a hipótese de que os coeficientes $\alpha$ e $\beta$ variam lentamente com o tempo, a fase $e^{2 i \chi}$ varia muito rápido em comparação com os coeficientes. Para isso $\chi$ tem que variar rapidamente. A condição para isso é dada por 
$\dot{\chi}=\omega(t) \ll\left|\frac{\dot{\omega}(t)}{\omega(t)}\right|$. Como uma primeira aproximação, vamos ignorar os termos oscilantes de (6.19). Levando em conta as condições de contorno dos coeficientes, obtemos valores aproximados para os coeficientes:

$$
\alpha^{(0)}(t)=\left[\frac{\omega}{\omega(t)}\right]^{1 / 2}, \beta^{(0)}(t)=0 .
$$

Com isso em mãos e sabendo que $(a-1) \frac{i \omega}{\pi}=\langle\omega|\mathcal{T}| \omega\rangle$, temos que:

$$
\langle\omega|\mathcal{T}| \omega\rangle=\frac{i \omega}{\pi}\left(\exp \left\{-i \int_{-\infty}^{+\infty} d t\left[\left(\omega^{2}+V(t)\right)^{1 / 2}-\omega\right]\right\}-1\right)
$$

Essa expressão é análoga à amplitude de espalhamento para aproximação semi-clássica do espalhamento frontal [107]. O espalhamento frontal não corresponde à criação de partículas, uma vez que a direção da onda incidente é a mesma da final, em analogia com o espalhamento de partículas. A criação de partículas corresponde a uma amplitude de espalhamento para trás, $\langle-\omega|\mathcal{T}| \omega\rangle$ diferente de zero, o que não acontece nessa primeira aproximação. Assim, a altas energias, essa primeira aproximação não leva à criação de partículas: para computarmos nosso efeito precisamos ir além.

Para isso, vamos resolver a segunda equação de (6.19), substituindo $\alpha$ por seu valor aproximado $\alpha^{(0)}(t)$, em (6.20), em que obtemos um expressão para a derivada de $\beta$ que pode facilmente ser resolvida fornecendo:

$$
\beta=-\int_{t}^{+\infty} d t^{\prime} \frac{\dot{\omega}\left(t^{\prime}\right)}{2 \omega\left(t^{\prime}\right)} e^{-2 i \chi\left(t^{\prime}\right)} .
$$

Para podermos calcular a probabilidade por unidade de volume de não criar partículas, vemos que precisamos determinar o módulo da amplitude de espalhamento para trás. Com $\beta$ dado acima, utilizando (6.15), temos que $|\langle-\omega|\mathcal{T}| \omega\rangle|=\frac{\omega}{\pi}|\beta(-\infty)|$, o que segundo a equação (6.5) fornece a probabilidade:

$$
\frac{d W}{d V}=\int \frac{d^{3} p}{(2 \pi)^{3}}\left|\int_{-\infty}^{+\infty} d t^{\prime} \frac{\dot{\omega}\left(t^{\prime}\right)}{2 \omega\left(t^{\prime}\right)} e^{-2 i \chi\left(t^{\prime}\right)}\right|^{2}
$$

Retomando a nossa probabilidade por unidade de volume e de tempo, escrevemos explicitamente a hipótese de que o campo é ligado e desligado (adiabaticamente) em instantes finitos, $-T / 2$ e $T / 2$. Então, a taxa de criação de pares é dada por: 


$$
\begin{aligned}
\omega & =\frac{d W}{d V d T} \\
& =\lim _{T \rightarrow \infty} \frac{1}{T} \int \frac{d^{3} p}{(2 \pi)^{3}}\left|\int_{T / 2}^{+T / 2} d t^{\prime} \frac{\dot{\omega}\left(t^{\prime}\right)}{2 \omega\left(t^{\prime}\right)} e^{-2 i \chi\left(t^{\prime}\right)}\right|
\end{aligned}
$$

Podemos, agora, colocar a forma explícita do campo externo oscilatório que vamos utilizar. Esse campo é um campo puramente elétrico da forma $E(t)=E_{0} \operatorname{sen}\left(\omega_{0} t\right)$. Seu potencial, portanto é $A(t)=\frac{E_{0}}{\omega_{0}} \cos \left(\omega_{0} t\right)$.

Em posse do formato do potencial, podemos substituí-lo na freqüência variável,

$$
\omega(t)=\left[m^{2}+p_{\perp}^{2}+\left(p_{3}-\frac{e E_{0}}{\omega_{0}} \cos \omega_{0} t\right)^{2}\right]^{1 / 2} .
$$

Substituindo essa freqüência em $\chi(t)$, podemos integrá-la:

$$
\begin{aligned}
\chi(t) & =\int_{0}^{t} d t^{\prime} \omega\left(t^{\prime}\right) \\
& =t \underbrace{\int_{0}^{2 \pi} \frac{d\left(\omega_{0} t\right)}{2 \pi}\left[m^{2}+p_{\perp}^{2}+\left(p_{3}-\frac{e E_{0}}{\omega_{0}} \cos \omega_{0} t\right)^{2}\right]^{1 / 2}}_{\Omega} \\
& +\underbrace{\int_{2 \pi}^{t} \frac{d\left(\omega_{0} t\right)}{2 \pi}\left[m^{2}+p_{\perp}^{2}+\left(p_{3}-\frac{e E_{0}}{\omega_{0}} \cos \omega_{0} t\right)^{2}\right]^{1 / 2}}_{\Phi(t)} \\
& =t \Omega+\Phi(t),
\end{aligned}
$$

em que a função $\Phi(t)$ representa a parte periódica e $\Omega$ é chamada de freqüência renormalizada.

Substituindo essa decomposição na taxa de criação de partículas (6.24) e expandindo a parte periódica em uma série de Fourier, $\frac{\dot{\omega}(t)}{2 \omega(t)} e^{-2 i \Phi(t)}=\sum_{n=-\infty}^{\infty} c_{n} e^{i n \omega_{0} t}$, podemos realizar as integrais obtendo: 


$$
\begin{aligned}
\omega & =\lim _{T \rightarrow \infty} \frac{1}{T} \sum_{n=-\infty}^{+\infty} \int \frac{d^{3} p}{(2 \pi)^{3}}\left|\int_{-T / 2}^{+T / 2} d t^{\prime} c_{n} e^{i n \omega_{0} t} e^{-2 i \Omega\left(t^{\prime}\right)}\right| \\
& =\omega_{0} \int \frac{d^{3} p}{(2 \pi)^{2}} \sum_{n=-\infty}^{+\infty} \delta\left(n-\frac{2 \Omega}{\omega_{0}}\right)\left|c_{n}\right|^{2} \\
& \cong \omega_{0} \int \frac{d^{3} p}{(2 \pi)^{2}} \frac{\left|c_{\xi}\right|^{2}}{\omega_{0}}=\omega_{0} \frac{e E}{2} \int \frac{d^{3} p}{(2 \pi)^{2}} \frac{|c|^{2}}{\omega_{0}}
\end{aligned}
$$

em que, na penúltima passagem, dado que $\Omega / \omega_{0} \gtrsim m / \omega_{0} \gg 1$, então podemos substituir a soma discreta em $n$ por uma integral e, sendo $\xi=\frac{2 \Omega}{\omega_{0}}$, renomeamos $c_{\xi}=\frac{e E}{2} c$. $\mathrm{O}$ coeficiente de Fourier pode ser obtido da seguinte maneira: chamando,

$$
f\left(\omega_{0} t\right)=\frac{\dot{\omega}(t)}{2 \omega(t)} e^{-2 i \Phi(t)}=\sum_{n=-\infty}^{\infty} c_{n} e^{i n \omega_{0} t}
$$

a expansão de Fourier, seu coeficiente é dado, substituindo $n \rightarrow 2 \Omega / \omega_{0}$, por:

$$
c_{\xi}=\frac{1}{2 \pi} \int_{-\pi}^{\pi} d x f(x) e^{-i \frac{2 \Omega}{\omega_{0}} x}, x=\omega_{0} t .
$$

Logo, como:

$$
f(x)=\frac{\operatorname{sen} x\left(p_{3}-\frac{e E_{0}}{\omega_{0}} \cos x\right)}{\left[m^{2}+p_{\perp}^{2}+\left(p_{3}-\frac{e E_{0}}{\omega_{0}} \cos \omega_{0} t\right)^{2}\right]} e^{-2 i \Phi(t)}
$$

o coeficiente é dado por:

$$
\begin{aligned}
c & =\int_{-\pi}^{\pi} \frac{d x}{2 \pi} \frac{\operatorname{sen} x\left(p_{3}-\frac{e E_{0}}{\omega_{0}} \cos x\right)}{\left[m^{2}+p_{\perp}^{2}+\left(p_{3}-\frac{e E_{0}}{\omega_{0}} \cos \omega_{0} t\right)^{2}\right]} e^{-2 i[t \Omega+\Phi(t)]} \\
& \times \exp \left\{\frac{-2 i}{\omega_{0}} \int_{0}^{x} d x^{\prime}\left[m^{2}+p_{\perp}^{2}+\left(p_{3}-\frac{e E_{0}}{\omega_{0}} \cos \omega_{0} t\right)^{2}\right]^{1 / 2}\right\},
\end{aligned}
$$

Para podermos calcular a taxa de criação de partículas precisamos determinar $|c|^{2}$. Para isso, é preciso calcular a integral em $c$ de uma função periódica com período $2 \pi$ (note-se que $2 \Omega / \omega_{0}$ é inteiro). Essa integral é feita no Apêndice B. Na integração fizemos $c=2 i \operatorname{Im} c_{1}$. Como só queremos a parte imaginária de $c_{1}$, vamos separar $\nu$ em uma parte real e imaginária, $\int_{0}^{x_{0}} \nu=-A+i B$ e como obtemos que a integral em $c_{1}$ é dada por: 


$$
\int_{\widetilde{\Gamma}_{1}} \frac{d y}{2 \pi y} \exp \left(y^{3 / 2}\right)=\frac{2}{3} \oint \frac{d u}{2 \pi u} e^{u}=\frac{2}{3} i,
$$

temos que a expressão para a taxa de criação é dada por:

$$
\omega=\frac{1}{9} \omega_{0} \int \frac{d^{3} p}{(2 \pi)^{2}} e^{-2 A} \cos ^{2} B .
$$

Podemos analisar em (6.33) que a exponencial decrescente indica que os pares tendem a ser emitidos com momento pequeno. Com isso podemos estimar essa taxa seguindo os seguintes passos: $p_{3}$ é colocado igual a zero e $\cos ^{2} B$ é substituído por seu valor médio $1 / 2$. Essas simplificações equivalem a multiplicar o resultado por um fator numérico. Levando em conta essas simplificações, de (B.1), temos que:

$$
x_{0}=\frac{1}{2} \pi+i \sinh ^{-1}[\underbrace{\left(\frac{\omega_{0}}{e E}\right)\left(m^{2}+p_{\perp}^{2}\right)^{1 / 2}}_{y}] .
$$

Para o cálculo de A, utilizando o limite de integração $x_{0}$ dado, obtemos:

$$
A=\frac{2\left(m^{2}+p_{\perp}^{2}\right)}{e E} \int_{0}^{1} d y\left[\frac{1-y^{2}}{1+y^{2} \underbrace{\omega_{0}^{2}\left(m^{2}+p_{\perp}^{2}\right) / e^{2} E^{2}}_{z}}\right],
$$

em que definimos a função:

$$
g(z)=\frac{4}{\pi} \int_{0}^{1} d y \frac{1-y^{2}}{1+z^{2} y^{2}} .
$$

A normalização dessa função é escolhida de forma que $g(0)=1$. Logo, fazendo a substituição $\left(m^{2}+p_{\perp}^{2}\right)=\left(e E / \omega_{0}\right)^{2} u^{2}$, e escrevendo o cosseno como seu valor médio, determinamos a forma da taxa de criação de partículas:

$$
\omega=\frac{(e E)^{3}}{18 \pi \omega_{0}^{2}} \int_{\gamma}^{\infty} d u u \exp \left[-\frac{\pi e E}{\omega_{0}^{2}} u^{2} g(u)\right],
$$

em que o parâmetro $\gamma=m \omega_{0} / e E$ é definido. Esse parâmetro é a razão entre duas quantidades pequenas, que mencionamos no início da seção, $\left(\omega_{0} / m\right)$ e $\left(e E / m^{2}\right)$, e é importante quantificar o seu tamanho. A função $u^{2} g(u)$ é monotonicamente crescente em relação ao sinal de integração acima. Dessa forma podemos ver que:

$$
\frac{e E}{\omega_{0}^{2}} u^{2} g(u) \geq \frac{e E}{\omega_{0}^{2}} \gamma^{2} g(\gamma)=\frac{m^{2}}{e E} g(\gamma) \gg 1
$$


Com esse limite é possível fazer a integração na variável $u$, o que leva ao resultado final:

$$
\omega=\frac{\alpha E^{2}}{2 \pi} \frac{1}{g(\gamma)+\frac{1}{2} \gamma g^{\prime}(\gamma)} \exp \left[-\frac{\pi m^{2}}{e E} g(\gamma)\right]
$$

Esse resultado mostra a probabilidade por unidade de volume e por unidade de tempo de não criar partículas. Podemos ver que ela depende da freqüência do campo, em $\gamma$. Com isso, a partir de $\gamma$, podemos verificar o comportamento da solução para cada limite: o limite de campo forte e baixa freqüência que é o caso do campo constante em que $\gamma \ll 1$ ; o limite de campo fraco e alta freqüência que é o limite perturbativo com $\gamma \gg 1$. Na Figura 6.1, podemos ver como a função $g(\gamma)$ se comporta nesses limites.

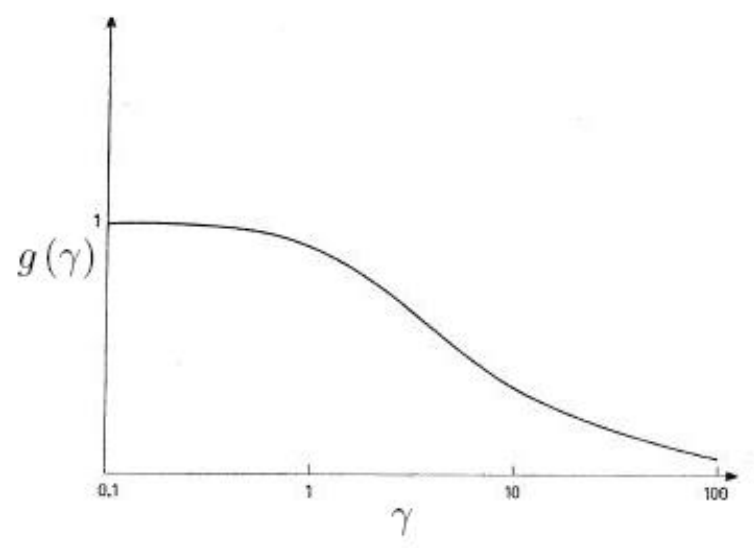

Figura 6.1: Curva da função que determina os limites, $g(\gamma)$, retirada de [74].

\section{Limites}

Podemos aproximar de forma uniforme a função $g$ por $g(z) \simeq \frac{4}{\pi \gamma} \sinh ^{-1} \gamma$. Para o limite em que $\gamma$ é pequeno, essa expressão diverge por um fator $\frac{1}{4} \pi$. Assim, para cada um desses limites, podemos derivar a partir de (6.39) a probabilidade de não emitir pares:

$$
\begin{aligned}
& \omega \simeq \frac{\alpha E^{2}}{2 \pi} \exp \left(-\frac{\pi m^{2}}{e E}\right), \gamma \ll 1 \\
& \omega \simeq \frac{\alpha E^{2}}{8}\left(\frac{e E}{2 m \omega_{0}}\right)^{4 m / \omega_{0}}, \gamma \gg 1 .
\end{aligned}
$$

Veremos que esse resultado retoma o resultado do efeito Schwinger estático no limite de baixas freqüências e campo alto, exceto por um fator $\pi$. No limite de campo baixo e freqüências altas, obtemos uma probabilidade proporcional ao potencial elevado a uma potência igual ao número mínimo de fótons necessários para se produzir um par, $2 \mathrm{~m} / \omega_{0}$.

Com isso, podemos verificar os limites experimentais necessários para que se observe 
criação de pares. Para observarmos pares, o fator na exponencial de (6.39) deve ser menor do que um, caso contrário a criação de partículas é exponencialmente amortecida. Com isso, a condição para a criação de partículas é:

$$
e E \gtrsim \pi m^{2} g(\gamma)
$$

ou, se substituirmos a aproximação para a função mencionada acima:

$$
e E \gtrsim \frac{m \omega_{0} c}{\sinh \left(\hbar \omega_{0} / 4 m c^{2}\right)}
$$

em que recolocamos as contantes $\hbar$, a velocidade da luz, c, anteriormente considerados iguais a um, mas que são necessárias para uma previsão numérica do limite de criação de partículas.

\subsubsection{Tentativas e propostas experimentais}

Apesar de, com a tecnologia atual de lasers, ainda não ser possível atingir os limites de criação de partícula para o efeito Schwinger, tanto estático quanto oscilatório, muito esforço tem sido investido em mecanismos que possam diminuir essa energia, ou freqüência necessária. Apresentamos aqui um panorama resumido das tentativas de experimentação do efeito Schwinger, que inclui também tentativas de medições indiretas.

Podemos reconhecer até hoje dois tipos de experimentos na terra e um astrofísico, de verificação indireta, que foram propostos para identificar a polarização do vácuo eletromagnético por um campo elétrico supercrítico, o efeito Schwinger.

1. Em colisões centrais de íons pesados perto da barreira de Coulomb, como proposto por Gerstein et al [148]. Essa proposta falhou devido ao pequeno tempo de contato dos íons que colidem.

2. Em colisões de um feixe de elétrons por um laser óptico. Um teste feito no SLAC (Stanford Linear Accelerator Center) [149], não deixou clara a evidência de que houve polarização do vácuo em um experimento de produção de pósitrons, [150], na colisão de 46, $6 \mathrm{GeV} / \mathrm{c}$ elétrons com pulsos ópticos de laser com potência da ordem de terawatts. Em [149], o experimento foi interpretado como um espalhamento luz-luz de multiplos fótons e explicado alternativamente como o efeito Schwinger em [150]. Ver também a referência [151].

3. Em torno de um buraco negro eletromagnético (EMBH), em um fenômeno observado como explosões de raios-gama [152].

Com o atual desenvolvimento da tecnologia de lasers, a verificação experimental do efeito Schwinger (dinâmico) voltou a despertar o interesse da comunidade científica . Apesar 
dessa tecnologia ainda não atingir os valores necessários, os projetos futuros de construção de lasers mostram-se promissores e os esforços teóricos para o cálculo de configurações desses lasers, que possibilitem a observação do efeito Schwinger, estão sendo feitos. Alguns laboratórios já anunciam a possibilidade de observação do efeito e novos experimentos são propostos.

No DESY (Deutsches Elektronen-Synchrotron) e no SLAC está em construção o XFEL (ou LCLS, no SLAC), um laser de elétrons livres de Raios-X. Espera-se que, otimizando as configurações experimentais, seja possível a observação do efeito Schwinger no futuro. Muitos cálculos teóricos estão sendo feitos para esse experimento, por Popov [153], R. Alkofer et al. (2001) [154], entre outros. Outros laboratórios que anunciaram a possibilidade da verificação do efeito Schwinger são o ELI [128] e o GSI, com o laser PHELIX [129], ambos em construção.

Um modelo que visa diminuir a energia necessária para a verificação do efeito Schwinger foi proposto por R. Schützhold (2008) [130]. Nele estuda-se a criação de partículas do vácuo de Dirac induzida por um campo elétrico forte e que varia lentamente (efeito Schwinger estático), que é sobreposto por um campo elétrico fraco e rapidamente variável (Efeito Schwinger dinâmico). Seu impacto combinado aumenta significativamente a taxa de criação de partículas no regime subcrítico, regime no qual os dois efeitos separados seriam fortemente suprimidos. Ilustrando esse mecanismo, pensemos que ele age da seguinte forma: o campo elétrico forte diminui a barreira para a criação dinâmica de partículas ou, alternativamente, o campo elétrico fraco gera sementes adicionais para o mecanismo de Schwinger estático. Esse mecanismo da combinação dos dois efeitos pode ser muito importante para a observação do efeito Schwinger nessa nova geração de lasers.

Alguns autores afirmam que o efeito Schwinger será primeiramente observado de maneira indireta em sistemas astrofísicos como estrelas de quarks [131] e estrelas de nêutrons [132].

\subsection{Efeito Schwinger Oscilatório via transformadas de Bogoliubov}

Partiremos agora para a descrição do ESO via transformadas de Bogoliubov. Nosso interesse com a descrição do efeito por esse formalismo é, além de demonstrar um método mais direto para o cálculo do ESO, fazer a analogia com sistemas submetidos a campos gravitacionais, ou métricas não estáticas. Essa descrição também faz um paralelo com a descrição do ECD, que será apresentada na próxima seção. Dessa forma, faremos aqui os cálculos somente para o campo escalar.

Nesta seção apresentaremos o cálculo do ESO utilizando o método das transformadas de Bogoliubov. Vamos utilizar o formalismo já desenvolvido na Subseção 5.1 para 
a interação de um campo escalar complexo com um potencial elétrico. Contudo, nesse caso, utilizaremos um campo elétrico oscilatório. A periodicidade que aparece nas equações de movimento leva à ressonância paramétrica do sistema, que é melhor descrita se transformarmos essas equações em uma equação de Mathieu. Esse será nosso ponto de partida para estudarmos a evolução do sistema, em particular do número de pares criados, utilizando as transformações de Bogoliubov e a aproximação semi-clássica.

Vamos considerar um sistema em que um campo escalar complexo está interagindo com um potencial elétrico oscilando no tempo na direção $x_{3}$, da forma:

$$
A=(0,0,0, A(t)), \quad \operatorname{com} A(t)=\frac{E_{0}}{\omega_{0}} \operatorname{sen}\left(\omega_{0} t\right) .
$$

Aplicando esse potencial nas equações de movimento (5.10), da Subseção 5.1, eles ficam da forma:

$$
\ddot{\phi}_{\mathbf{k}}+\omega_{\mathbf{k}}^{2}(t) \phi_{\mathbf{k}}=0
$$

em que a dependência temporal da freqüência é dada por:

$$
\begin{aligned}
\omega_{\mathbf{k}}^{2}(t) & =k^{2}+m^{2}+2 e k_{3} \frac{E_{0}}{\omega_{0}} \operatorname{sen}\left(\omega_{0} t\right)-(i e)^{2}\left(\frac{E_{0}}{\omega_{0}} \operatorname{sen}\left(\omega_{0} t\right)\right)^{2} \\
& =k_{\perp}^{2}+m^{2}+\left[k_{3}+\frac{e E_{0}}{\omega_{0}} \operatorname{sen}\left(\omega_{0} t\right)\right]^{2}
\end{aligned}
$$

A equação (6.44) com freqüência dada por (6.45) é conhecida como equação de Hill. Podemos ver isso se considerarmos $z=\omega_{0} t / 2$, em que obtemos:

$$
\frac{d^{2} \phi_{\mathbf{k}}}{d z^{2}}+\left[\theta_{0}+2 \theta \cos 2 z+\frac{1}{2} \vartheta \cos 4 z\right] \phi_{\mathbf{k}}=0,
$$

onde $\omega_{0}^{2} \theta_{0}=\left(k_{3}^{2}+\frac{e^{2} E_{0}^{2}}{2}\right), \theta=-\frac{4 e E_{0} k_{3}}{\omega_{0}^{2}}$ e $\vartheta=\frac{2 e E_{0}}{\omega}$.

Analisando (6.45), podemos ver que o termo $k_{3}+\frac{e E_{0}}{\omega_{0}} \operatorname{sen}\left(\omega_{0} t\right)$ nos parece fornecer um termo oscilante deslocado por um fator $k_{3}$ do eixo. Dessa forma, se fizermos $k_{3}=0$, estaremos simplesmente transladando o potencial para que seu mínimo seja em zero. Ao invés disso, podemos também utilizar a condição $\partial_{\mu} A^{\mu}=k_{3} A_{3}=0$, do calibre de Coulomb que adotamos inicialmente no Capítulo 4. Com isso, temos criação de partículas para todas as componentes de $k$, mas o termo $k_{3} A_{3}$ some da equação. Vamos utilizar essa última condição.

Esse argumento é confirmado matematicamente, uma vez que, na análise do comportamento da equação de Hill, utilizamos as equações de Mathieu para verificarmos os regimes estabilidade ou instabilidade. Isso é feito, pois essa análise quase não apresenta diferença para essas duas equações [133]. 
Assim, podemos reduzir nossa equação de movimento para os modos para uma equação da forma:

$$
\ddot{\phi}_{\mathbf{k}}+\left(k^{2}+m^{2}+\frac{e^{2} E_{0}^{2}}{\omega_{0}^{2}} \operatorname{sen}^{2}\left(\omega_{0} t\right)\right) \phi_{\mathbf{k}}=0
$$

em que a freqüência é dada por:

$$
\omega_{\mathbf{k}}^{2}(t)=k^{2}+m^{2}+\frac{e^{2} E_{0}^{2}}{\omega_{0}^{2}} \operatorname{sen}^{2}\left(\omega_{0} t\right) .
$$

Essa equação também descreve um oscilador com freqüência periodicamente variável, contudo somente contém os termos quadráticos da freqüência. Esse periodicidade pode levar a ressonâncias paramétricas para os modos com certos valores de $k$. Logo, para descrevermos melhor esse comportamento, podemos redefinir a variável $\omega_{0} t=z$, que faz com que a equação (6.47) se reduza a uma equação de Mathieu:

$$
\frac{d^{2} \phi_{\mathbf{k}}}{d z^{2}}+\left(A_{\mathbf{k}}-2 q \cos 2 z\right) \phi_{\mathbf{k}}=0
$$

em que $A_{\mathbf{k}}=\frac{\left(m^{2}+k^{2}\right)}{\omega_{0}^{2}}+2 q$ e $q=\left(\frac{e E_{0}}{2 \omega_{0}^{2}}\right)^{2}$. O fato de podermos reduzir nossa equação a uma equação de Mathieu nos traz informações sobre o sistema, uma vez que suas soluções exibem características especiais como uma estrutura de bandas de ressonância, determinadas pelos valores de $A$ e $q$.

Podemos observar aqui que, se ao invés de utilizarmos um potencial senoidal ilustrado na equação (6.43), tivéssemos utilizado um potencial também oscilatório, porém cossenoidal, como utilizado na Seção 6.1, teríamos as mesmas propriedades da criação de partículas em um potencial oscilatório, assim como poderíamos também reduzir o problema para a mesma equação de Mathieu acima, com a mesma transformação de variáveis, diferindo apenas por $A_{\mathbf{k}}=\frac{\left(m^{2}+k^{2}\right)}{\omega_{0}^{2}}-2 q$ e $q=-\left(\frac{e E_{0}}{2 \omega_{0}^{2}}\right)^{2}$.

O fato de podermos representar nosso problema em termos de uma equação de Mathieu nos permite analisar alguns comportamentos característicos das soluções [133]. Existe uma quantidade muito grande de soluções para esta equação, quer sejam periódicas e não-periódicas, que não podem ser escritas em termo de funções elementares. Como os coeficientes da equação são periódicos, aplica-se o teorema de Floquet, que afirma que uma equação diferencial com coeficientes periódicos admite uma solução periódica da forma:

$$
\phi_{\mathbf{k}}=u(z, \sigma) e^{\mu_{N} z},
$$

em que $\mu_{N}$ é o índice de Floquet e $\sigma$ é o valor de $k^{2}+m^{2}$ para o qual a solução é computada. Essas soluções apresentam uma estrutura de bandas de ressonância que, dependendo dos valores de $A$ e $q$, correspondem às bandas de instabilidade e estabilidade, Figura 6.2. 

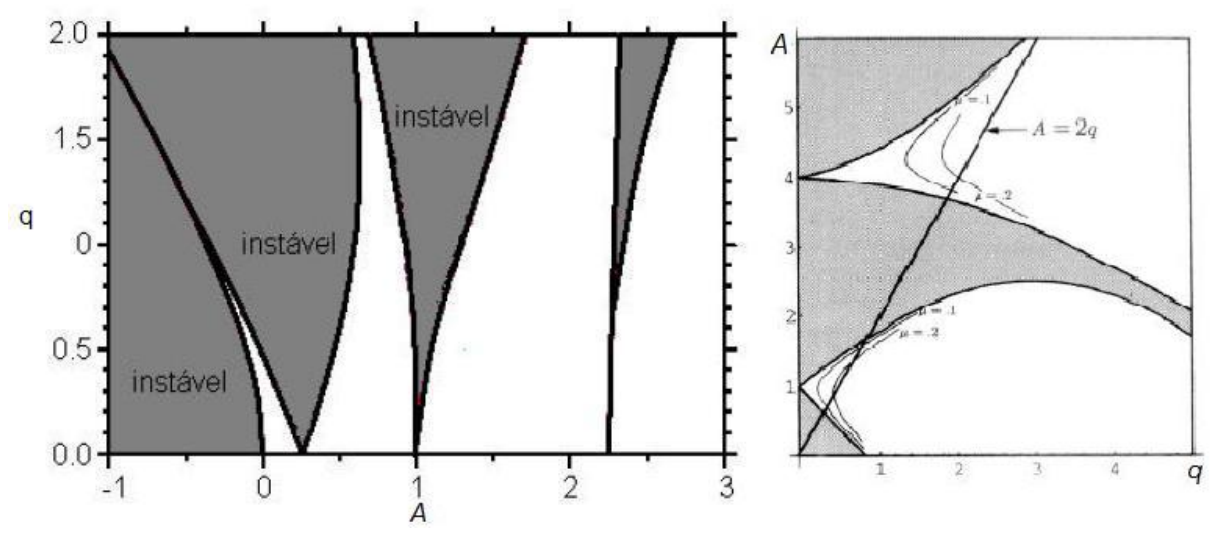

Figura 6.2: Espaço de fase da equação de Mathieu. Podemos ver a estrutura de bandas de instabilidade e estabilidade que as soluções apresentam. A escala do gráfico é arbitrária. O quadro a direita, retirado de [45], de $A \times q$, apresenta uma outra representação das bandas de instabilidade (em branco) e estabilidade (em cinza), em traçamos a linha $A=2 q$. Esse gráfico é o mesmo que o da esquerda rotacionado.

Nas bandas de instabilidade, temos uma ressonância estreita (narrow), em que $\frac{e E_{0}}{\omega_{0}}<$ $\omega_{0}$ ou $q \ll 1$, ou seja, a amplitude da oscilação é menor que a freqüência. Nesse regime os modos são amplificados exponencialmente. Para $\frac{e E_{0}}{\omega_{0}}>\omega_{0}$ ou $q \gg 1$, temos um regime de ressonância ampla (broad), em que a ressonância ocorre para uma faixa ampla de valores de $k$. Nesse regime vemos que a amplitude de oscilação é maior que sua freqüência.

Por outro lado, podemos ver que a condição para criação de partículas obtida na seção anterior, (6.42), corresponde a:

$$
\frac{e E_{0}}{\omega_{0}^{2}} \gtrsim 4 \underbrace{\frac{m^{2}}{\omega_{0}^{2}}}_{\gg 1} \gg 1
$$

em que impusemos a mesma condição da seção anterior de que $\omega_{0} / m \ll 1$. Com essa condição podemos expandir em primeira ordem o senh de (6.42),

$$
\frac{e E_{0}}{\omega_{0}^{2}}>\frac{m c}{\omega_{0} \operatorname{senh}\left(\hbar \omega_{0} / 4 m c^{2}\right)} \simeq \frac{m c}{\omega_{0} \hbar \omega_{0} / 4 m c^{2}} \simeq \frac{4 m^{2}}{\omega_{0}^{2}} .
$$

Podemos utilizar o resultado obtido para férmions no caso de bósons, uma vez que, como visto no Capítulo 5 , eles diferenciam somente por uma constante multiplicativa.

Ou seja, a condição de criação de partículas corresponde à condição de ressonância ampla (broad). Isso nos mostra que a criação de partículas somente é eficiente no regime de ressonância ampla. Essa equivalência é de muita importância, uma vez que é uma confirmação dessas duas descrições, pois a criação somente é detectada experimentalmente 
nos limites em que a criação é mais pronunciada, de ressonância paramétrica. Assim, vamos estudar somente esse regime, que não pode ser resolvido pelos métodos usuais de resolução do caso da ressonância estreita (narrow) [133].

Uma vez que estabelecemos o problema, agora temos que resolvê-lo. A estratégia desenvolvida foi utilizar um método analítico para a resolução aproximada de (6.49), ao invés de resolver a equação de Mathieu e analisar as soluções baseadas nos gráficos padrão de estabilidade/instabilidade. O método desenvolvido, além de reproduzir as soluções de (6.49) no regime de ressonância broad, também nos fornece uma percepção física mais interessante da ressonância paramétrica. Apesar de já ter sido utilizado para a determinação da criação de partículas em [46], apresentamos aqui sua primeira utilização no efeito Schwinger oscilatório.

Esse método basea-se no fato de que $\phi_{\mathbf{k}}$ tem evolução adiabática e o número de partículas não cresce entre os instantes em que o campo externo aplicado é igual a zero, $A\left(t_{j}\right)=0, j=1,2,3 \ldots$ Assim, na vizinhança desses instantes o campo tem evolução não-adiabática. Com isso, podemos formular a teoria da criação de partículas em um campo oscilatório em termos da aproximação adiabática ou semi-clássica. Conectamos assim os resultados em cada um desses instantes por transformadas de Bogoliubov, o que nos permite calcular o número de partículas criadas.

Como podemos ver pela forma do potencial oscilante aplicado, $A(t)=\frac{E_{0}}{\omega_{0}} \operatorname{sen}\left(\omega_{0} t\right)$ é igual a zero quando $\omega_{0} t_{j}=\pi j, j=1,2,3, \ldots$. Nestes mesmos instantes $t_{j}$, a freqüência (6.48) atinge seu mínimo, dado por $\sqrt{k^{2}+m^{2}}$. Então, podemos verificar que, para potenciais $A(t)$ muito pequenos, $\dot{\omega} / \omega^{2} \gtrsim 1$ (ou seja, quando a freqüência varia muito rapidamente), a mudança na freqüência deixa de ser adiabática. Isso pode ser visto também, e de maneira mais precisa, com ajuda do parâmetro adiabático, ou parâmetro WKB, definido na Seção 5.2.2, equações (5.127) e (5.128). Assim, nos instantes (e em sua vizinhança) em que o potencial oscilante aplicado é zero, temos a violação da adiabaticidade, $\left|Q / \omega^{2}\right| \gtrsim 1$, como podemos ver na Figura 6.3. São nesses instantes que temos a criação de partículas. 


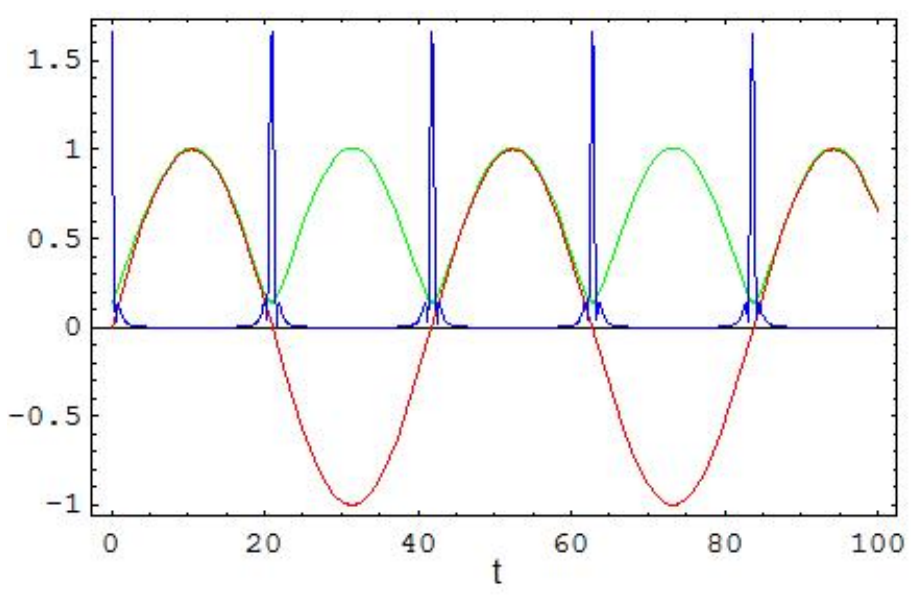

Figura 6.3: Mostramos nesse gráfico, em vermelho, o formato do potencial externo aplicado. Em verde representamos a freqüência e em azul o parâmetro WKB (divido por 30 para ser compatível com a escala). Pode-se observar que nos instantes em que o campo é muito pequeno, temos a violação da adiabaticidade (WKB). Nesses instantes é que ocorre a criação de partículas.

Temos então que entre os instantes $t_{j}$ temos estados adiabáticos. Isso significa que, na aproximação semi-clássica, quando a freqüência muda adiabaticamente, o número de partículas, $n_{\mathbf{k}}$, é um bom parâmetro para descrever o sistema, uma vez que é um invariante adiabático. Logo, $n_{\mathbf{k}}$ varia somente nesses pequenos intervalos de tempo em que a adiabaticidade é quebrada. Com isso, vamos utilizar a técnica desenvolvida na Seção 5.2.2 em que esses estados adiabáticos serão considerados os estados in e out para cada $t_{j}$ e podemos conectar esses estados por uma transformação de Bogoliubov. O número de partículas é calculado através desses coeficientes.

$\mathrm{Na}$ vizinhança dos instantes $t_{j}$, a equação geral do sistema (6.47) pode ser aproximada por:

$$
\ddot{\phi}_{\mathbf{k}}+\left(k^{2}+m^{2}+e^{2} E_{0}^{2}\left(t-t_{j}\right)^{2}\right) \phi_{\mathbf{k}}=0
$$

uma vez que $\left(t-t_{j}\right)$ na vizinhança é pequeno. Podemos fazer uma transformação de variáveis: $\tau=\sqrt{e E_{0}}\left(t-t_{j}\right)$ e $\Upsilon=\frac{\left(\mathbf{k}^{2}+m^{2}\right)}{e E_{0}}$, que nos fornece, para cada $j$ :

$$
\frac{d^{2} \phi_{\mathbf{k}}}{d \tau^{2}}+\underbrace{\left(\Upsilon+\tau_{j}^{2}\right)}_{\omega^{2}(\tau)} \phi_{\mathbf{k}}=0,
$$

que é equivalente à equação (5.125). Com isso, reduzimos nosso problema da resolução de uma equação de Mathieu com potencial oscilatório para um problema de espalhamento 
entre potenciais parabólicos (espalhamento em $t_{j}$ ).

A utilização do método WKB não pode ser considerada para um potencial oscilante. Contudo, utilizando esse método de espalhamento em potenciais parabólicos consideramos o sistema oscilatório como um sequência de sistemas em que é possível utilizarmos o método WKB.

Vamos considerar a primeira oscilação do campo externo, em torno de $j=1$. Sabemos que nos instantes anteriores e posteriores de $t_{1}$ temos estados adiabáticos. Sabemos também, da Seção 5.2.2, que para definirmos o número de partículas criadas precisamos somente definir o estado inicial, desde que o final seja também adiabático. Com isso, definimos o estado in como sendo o vácuo adiabático, descrito por:

$$
\phi_{w k b, \mathbf{k}}=\frac{1}{\sqrt{2 \omega}} \exp \left(-i \int_{\tau_{i n i}}^{\tau} \omega\left(\tau^{\prime}\right) d \tau^{\prime}\right)
$$

que é solução de (5.129) no instante in.

Podemos ver aqui claramente que reduzimos nosso problema àquele da Seção 5.2.2, para cada oscilação do campo externo. Assim, como feito anteriormente, nos instantes em que a condição de adiabaticidade é válida, a solução do problema é dada pela aproximação:

$$
\phi_{\mathbf{k}}^{(1)}(\tau) \simeq \alpha_{k}^{(0)} \phi_{w k b, \mathbf{k}}(\tau)+\beta_{k}^{*(0)} \phi_{w k b, \mathbf{k}}^{*}(\tau)
$$

em que esses instantes são menores que $t_{2}$ e $\alpha_{k}^{(0)}$ e $\beta_{k}^{(0)}$ são os coeficientes de Bogoliubov, $\operatorname{com}\left|\alpha_{\mathbf{k}}^{(0)}\right|^{2}-\left|\beta_{\mathbf{k}}^{(0)}\right|^{2}=1$. O rótulo (0) nos coeficientes de Bogoliubov acima denotam que essa é a primeira oscilação do campo.

Como nosso estado inicial é o vácuo adiabático, temos que $\alpha_{k}=1$ e $\beta_{k}=0$ nesse limite $\left(\lim _{\tau / \sqrt{\Upsilon} \rightarrow-\infty} \phi_{\mathbf{k}}^{(0)}=\phi_{w k b, \mathbf{k}}\right)$, e portanto, o número de partículas é dado por:

$$
N_{\mathbf{k}}^{(1)}=\left|\beta_{k}^{(0)}\right|^{2}
$$

que representa a criação espontânea de partículas do vácuo, em que o coeficiente de Bogoliubov é dado por:

$$
\left|\beta_{\mathrm{k}}^{(0)}\right|^{2}=\mathrm{e}^{-\pi \Upsilon}
$$

como calculado na Seção 5.2.2.

Se considerarmos agora o instante $t_{j}$, passadas $j$ oscilações do campo externo, sabendo que antes e depois da (vizinhança de) $t_{j}$ temos estados adiabáticos, em analogia com o feito acima para a primeira oscilação, temos que o campo $\phi_{\mathbf{k}}$ antes do espalhamento em $t_{j}$, é dado por:

$$
\phi_{\mathbf{k}}^{(j)}(\tau) \simeq \alpha_{k}^{(j)} \phi_{w k b, \mathbf{k}}(\tau)+\beta_{k}^{*(j)} \phi_{w k b, \mathbf{k}}^{*}(\tau)
$$


em que os coeficientes $\alpha_{k}^{(j)}$ e $\beta_{k}^{(j)}$ são constantes no intervalo $t_{j-1}<t<t_{j}$. Logo, após o espalhamento, no intervalo $t_{j}<t<t_{j+1}$, o campo $\phi_{\mathbf{k}}$ tem a forma:

$$
\phi_{\mathbf{k}}^{(j+1)}(\tau) \simeq \alpha_{\mathbf{k}}^{(j+1)} \phi_{w k b, \mathbf{k}}(\tau)+\beta_{\mathbf{k}}^{*(j+1)} \phi_{w k b, \mathbf{k}}^{*}(\tau)
$$

em que $\alpha_{k}^{(j+1)}$ e $\beta_{k}^{(j+1)}$ são constantes no intervalo $t_{j}<t<t_{j+1}$.

É interessante reescrever esse resultado em função dos coeficientes de Bogoliubov iniciais, $\alpha_{\mathbf{k}}^{(0)}$ e $\beta_{\mathbf{k}}^{(0)}$, que são conhecidos. Com isso, podemos construir a formula de recorrência para o campo:

$$
\phi_{\mathbf{k}}^{(j+1)}(\tau) \simeq \alpha_{\mathbf{k}}^{(0)} \phi_{\mathbf{k}}^{(j)}(\tau)+\beta_{\mathbf{k}}^{*(0)} \phi_{\mathbf{k}}^{j *}(\tau)
$$

e, logo, para os coeficientes

$$
\left(\begin{array}{c}
\alpha_{\mathbf{k}}^{(j+1)} \\
\beta_{\mathbf{k}}^{(j+1)}
\end{array}\right)=\left(\begin{array}{cc}
\alpha_{\mathbf{k}}^{(0)} & \beta_{\mathbf{k}}^{*(0)} \\
\beta_{\mathbf{k}}^{(0)} & \alpha_{\mathbf{k}}^{*(0)}
\end{array}\right)\left(\begin{array}{c}
\alpha_{\mathbf{k}}^{(j)} \\
\beta_{\mathbf{k}}^{(j)}
\end{array}\right) .
$$

Por meio dessas relações de recorrência, o número de partículas criadas é dado por:

$$
\begin{aligned}
n_{\mathbf{k}}^{j+1} & =\left|\beta_{\mathbf{k}}^{(j+1)}\right|^{2} \\
& =n_{\mathbf{k}}^{j}+\left|\beta_{\mathbf{k}}^{(0)}\right|^{2}\left(1+2 n_{\mathbf{k}}^{j}\right)+\alpha_{\mathbf{k}}^{*(0)} \beta_{\mathbf{k}}^{(j)} \beta_{\mathbf{k}}^{*(0)} \alpha_{\mathbf{k}}^{(j)} \\
& =n_{\mathbf{k}}^{j}+\mathrm{e}^{-\pi \Upsilon}\left(1+2 n_{\mathbf{k}}^{j}\right)+\underbrace{\alpha_{\mathbf{k}}^{*(0)} \beta_{\mathbf{k}}^{(j)} \beta_{\mathbf{k}}^{*(0)} \alpha_{\mathbf{k}}^{(j)}}_{\text {termo de fase }}
\end{aligned}
$$

A equação acima coincide com a criação estimulada de partículas (5.94), calculada na Seção 5.2.2, com exceção de um termo de fase. Essa criação estimulada ocorre uma vez que as partículas criadas nos instantes anterior $j$ fazem com que o estado adiabático inicial seja composto de $n_{j}$ partículas que estimulam uma criação maior de partículas em $j+1$.

O termo de fase que aparece na equação (6.64) pode determinar se o número de partículas naquele espalhamento irá crescer ou decrescer. Fica claro que o número total de partículas sempre cresce, uma vez que temos bósons, como já discutido na Seção 5.2.2. Contudo, localmente para cada instante $j$, o valor da fase pode determinar se o número de partículas irá crescer ou decrescer, uma vez que o todo o problema depende da interferência das funções de onda, ou seja, da correlação ou anticorrelação da fase entre espalhamentos sucessivos. Isso ocorre uma vez que a criação de partículas em um campo externo variável não são autoestados do número de partículas do estado- in, mas sim um estado-in squeezed e, nesse caso, a interferência das funções de onda podem levar ao decrescimento do número de partículas. Uma vez com isso presente, iremos estudar na próxima seção esse mesmo problema, porém utilizando o formalismo de estados squeezed.

A estrutura dos resultados obtidos nos permite verificar que a criação de partículas, 
que ocorre a cada oscilação do campo externo aplicado, ocorre entre platôs de estados adiabáticos, nos quais o número de partículas aumenta. Ou seja, pela forma de (6.64), podemos ver que o número de partículas é uma função do tipo degrau, que cresce em $t_{j}$ e $t_{j+1}$ (e em torno de suas vizinhanças) e é constante entre esse pontos de espalhamento.

Podemos observar que, se o estado inicial adiabático ( $i n$ ) não fosse o vácuo, mas sim um estado adiabático populado de partículas, teríamos que na primeira criação de partículas já seria uma criação estimulada, da forma (6.64). As fórmulas de recorrência para as criações de ordem mais alta teriam a mesma forma, com a diferença que o número de partículas no estado anterior, responsável pela estimulação, seriam maiores, pois levariam em conta que a primeira criação de partículas considera o número de partículas inicial.

\subsubsection{Formalismo dos estados squeezed}

Analogamente ao feito na Seção 5.2.2.4, podemos aqui reescrever o problema em termos dos parâmetros de squeezing. O procedimento para o caso oscilatório é o mesmo feito no caso estático, sendo que utilizaremos a mesma parametrização dos coeficientes de Bogoliubov e a freqüência que iremos utilizar para o cálculo de (5.147) nesse caso é dada pela equação (6.48), ou seja, é oscilatória. Com isso, é possível graficar esse resultado em função do tempo, em que o número de partículas é dado por (5.148).

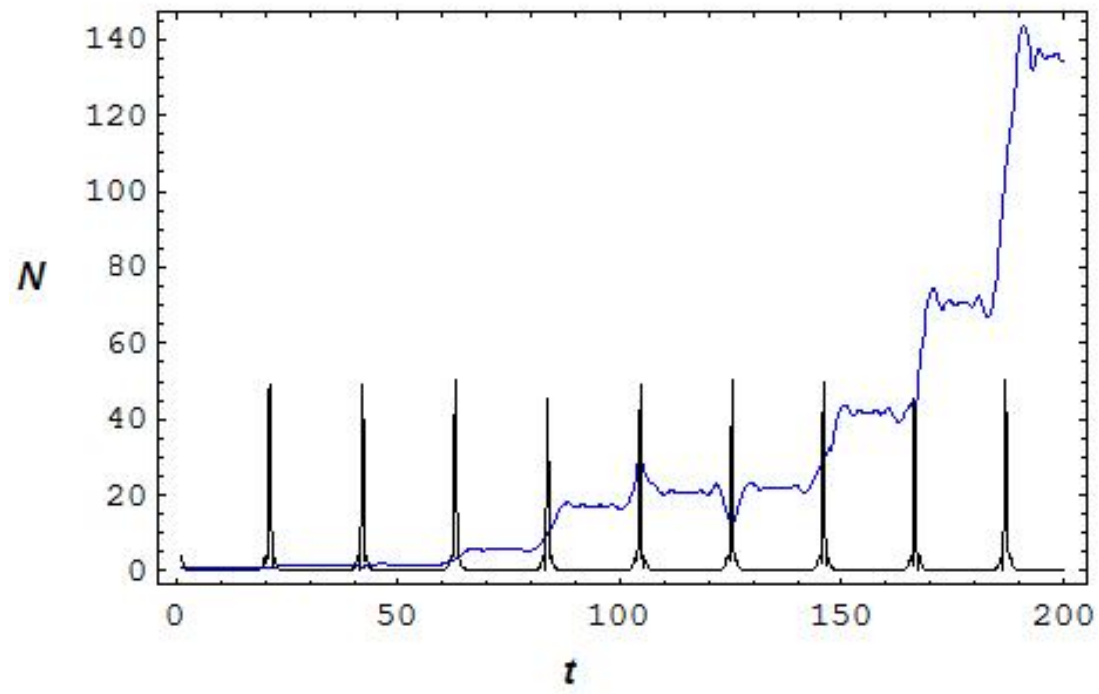

Figura 6.4: Número de partículas criadas no tempo. No gráfico observamos que as partículas são criadas nos instantes em que há violação do parâmetro WKB. 
Podemos ver na Figura 6.4 uma simulação do número de partículas em função do tempo. No mesmo gráfico foi colocado o parâmetro WKB, para que fosse possível verificar que as partículas são criadas exatamente nos instantes em que há violação WKB, ou violação da adiabaticidade. No gráfico podemos notar também que, como visto na Seção anterior, a criação de partículas ocorre em ciclos e tem um comportamento de degrau, ou seja, sempre ocorre entre dois instantes adiabáticos, em que a ação do campo é praticamente nula.

O número de partículas cresce em geral. Contudo, em alguns ciclos podemos ver que o número decresce, como mencionado na Seção 6.2 devido a fase do estado anterior, e aparece naturalmente no formalismo de estados squeezed.

Esse resultado será muito importante para a analogia com o pré-aquecimento, no Capítulo 7.

\subsection{Efeito Casimir Dinâmico}

O conhecido efeito Casimir, descoberto em 1948 por Casimir e Polder [135], é descrito como uma variação na energia de ponto zero do campo eletromagnético, que descreve o vácuo da QED. Casimir calculou essa força para o caso de duas placas condutoras colocadas no vácuo, o que ficou conhecido como efeito Casimir. Uma maneira de interpretar esse efeito é que a presença das placas modifica os modos do campo eletromagnético livre, através de um efeito de borda, ou da imposição de condições de contorno nessa borda. Ele também pode ser pensado como se o vácuo livre externo exercesse uma pressão nas placas, originando a força atratora.

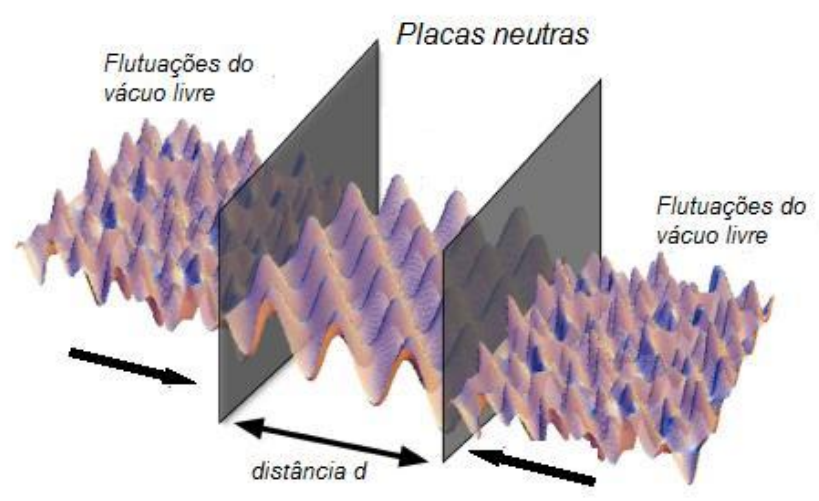

Figura 6.5: Esquema de [82] que mostra o Efeito Casimir. Podemos ver que as placas modificam os modos do campo eletromagnético entre as placas. O Efeito Casimir pode ser interpretado também como a pressão do vácuo externo as placas, o que gera a força de Casimir. 
Esse efeito ocorre devido às flutuações do vácuo quântico e é um efeito estático.

Uma generalização natural para o efeito Casimir estático é considerar situações em que as placas estejam em movimento acelerado. Esse efeito é chamado de efeito Casimir dinâmico, nome esse dado por J. Schwinger, em 1990. Quando as paredes são colocadas para oscilar, ou quando temos o caso de um espelho no vácuo em movimento acelerado, o vácuo quântico responde às condições de contorno dependentes do tempo que se anulam nos espelhos pela criação de pares de partículas reais vindas das flutuações do vácuo quântico. Conseqüentemente, espelhos perfeitos movendo-se no vácuo do campo eletromagnético induzem a criação de fótons. Como a criação de partículas e as forças de reação da radiação exercida pelo movimento do contorno são compatíveis, o nome efeito Casimir dinâmico é sugestivo também por esse ponto.

O primeiro cálculo de criação de partículas por uma fronteira móvel foi feito por G.T. Moore, em 1970 [136]. Ele calculou a taxa de criação de partículas em uma cavidade em que uma parede se matinha fixa e outra oscilava, em $1+1$ dimensões. Apesar de não apresentar resultados práticos, esse problema, utilizando eletrodinâmica escalar, foi importante para o estudo da solução geral dessa classe de modelos. Naquele trabalho, Moore concluiu que haveria criação de partículas, contudo essa criação seria muito pequena. Muitos trabalhos posteriormente dedicaram-se, baseados na descrição de Moore, à tarefa de aumentar essa taxa de criação para que o efeito fosse detectável.

Law, em 1994, considerou pela primeira vez condições de ressonância no ECD [137]. Essas condições amplificam muito a criação de partículas. No entanto, os primeiros cálculos foram feitos de maneira perturbativa ou numérica. Somente em 1996, com V.V. Dodonov e A.B. Klimov [147] foram feitos os primeiros cálculos analíticos do efeito, utilizando o formalismo das transformadas de Bogoliubov.

O problema da cavidade em uma dimensão é o modelo mais simplificado para se estudar esses problemas de TQC com condições de contorno. No caso de uma cavidade em três dimensões $(3+1$ dimensões) o problema é mais desafiador. A generalização do efeito em uma dimensão foi feita em [139, 140, 141, 142]. Esse problema é importante, uma vez que sua modelagem é mais próxima às situações reais de cavidade.

Um outro sistema, que consiste em um espelho (condutor) único movendo-se aceleradamente no vácuo, também é considerado um tipo de efeito Casimir dinâmico e foi considerado, principalmente por P.A. Maia Neto [143]. O ECD também foi estudado em diferentes geometrias [144].

Convém ressaltar que o ECD somente ocorre se o espelho estiver se movendo aceleradamente. Ou seja, qualquer espelho que se mova em um vácuo eletromagnético com velocidade constante, pela invariância de Lorentz, não cria fótons. Se o espelho se mover com aceleração constante, teremos a emissão de radiação de Unruh [145]. Assim, o caso em que a parede de uma cavidade oscila descreve bem o ECD.

O ECD pode ser demonstrado em $1+1$ dimensões, uma espacial e uma temporal, ou 
em $3+1$ dimensões, três espaciais e uma temporal. Vamos demostrar aqui o efeito em $1+1$ dimensões e em $3+1$ dimensões, ou seja, o movimento de uma parede de uma cavidade. Esse sistema é a descrição teórica simplificada do problema experimental que veremos abaixo.

\subsubsection{Quantização do campo escalar em 1+1 dimensões com con- dições de contorno}

Para familiarizarmo- nos com o problema de placas no vácuo com condições de contorno, em que se insere o ECD, vamos estudar o modelo simples que consiste em duas placas condutoras, ou espelhos, no vácuo de um campo escalar massivo em uma dimensão espacial e uma temporal [136, 147, 82, 139]. Queremos determinar as equações de movimento desse campo, em que são impostas condições de contorno dependente do tempo, devido ao movimento acelerado do espelho. Isso é fundamental para mostrarmos a maior diferença entre o ECD e os outros mecanismos de criação de partículas, como o efeito Schwinger ou a criação de partículas em campos gravitacionais (Inflação, pré-aquecimento, ...) : as equações de movimento dessa classe de problemas não se desacoplam. Uma conseqüência disso é que teremos um mecanismo a mais de criação de partículas para o ECD. Vemos, então, que esse simples modelo já nos permite visualizar as características mais importantes encontradas no ECD.

A densidade Lagrangeana que descreve um campo escalar massivo em duas dimensões, $\phi(t, x)$, é dada por:

$$
\mathcal{L}=\frac{1}{2}\left[\left(\frac{\partial \phi}{\partial t}\right)^{2}-\left(\frac{\partial \phi}{\partial x}\right)^{2}-m^{2} \phi^{2}\right]
$$

em que $m$ é a massa do campo. Com essa densidade, podemos escrever a ação de um campo,

$$
S=\frac{1}{2} \int d t \int_{I(t)} d x\left[\left(\frac{\partial \phi}{\partial t}\right)^{2}-\left(\frac{\partial \phi}{\partial x}\right)^{2}-m^{2} \phi^{2}\right]
$$

que está confinada em um intervalo dependente do tempo $I(t)=[0, l(t)]$. Por ser um problema com fronteiras, temos que impor condições de contorno para o campo nos pontos finais do intervalo. Diferentes condições podem ser impostas no problema, dependendo do sistema físico em consideração. Algumas das condições de contorno mais usadas nesse tipo de problema são: 


$$
\begin{aligned}
& \text { Dirichlet }: \phi(t, 0)=\phi(t, l(t))=0, \\
& \text { Neumann }: \frac{\partial}{\partial x} \phi(t, 0)=\frac{\partial}{\partial x} \phi(t, l(t))=0, \\
& \text { Mista(Dirichlet-Neumann) } \phi(t, 0)=\left\{\begin{array}{l}
\frac{\partial}{\partial x} \phi(t, l(t))=0, \text { ou } \\
\frac{\partial}{\partial x} \phi(t, 0)=\phi(t, l(t))=0 .
\end{array}\right.
\end{aligned}
$$

Vamos utilizar aqui condições de contorno de Dirichlet. Assim, a dinâmica do campo é descrita pelas equações:

$$
\left[\left(\frac{\partial}{\partial t}\right)^{2}-\left(\frac{\partial}{\partial x}\right)^{2}-m^{2}\right] \phi(t, x)=0, \text { com } \phi(t, 0)=\phi(t, l(t))=0
$$

que é a equação de movimento do campo com condições de contorno de Dirichlet. Para podermos descrever o problema em termos dos modos do campo, e, para que tenhamos uma formulação canônica do problema, introduzimos um conjunto de autofunções do operador Laplaciano, $-\partial_{x}^{2}$, reais, dependentes do tempo $\left\{\varphi_{n}(t, x)\right\}$, que obedecem à equação de autovalores:

$$
-\partial_{x}^{2} \varphi_{n}(t, x)=\omega_{n}^{2}(t) \varphi_{n}(t, x)
$$

no intervalo $I(t)$ com autovalores dependentes do tempo $\omega_{n}^{2}(t)$, e sujeitas às condições de contorno. Esse conjunto satisfaz as condições de ortonormalidade e completeza para qualquer tempo $t$.

Para determinarmos essas autofunções, supomos que as placas estejam paradas, em um intervalo estático $\left[0, l_{0}\right]$. O conjunto de autofunções que obedece a essas condições pode ser escrita da forma $\left\{\sqrt{\frac{2}{l_{0}}} \operatorname{sen}\left(\frac{n \pi}{l_{0}} x\right)\right\}$, com $n=1,2, \ldots$, com autovalores $\left(n \pi / l_{0}\right)^{2}$, e é denominada base instantânea. Utilizando esse resultado para o caso em que a placa está em movimento, as autofunções têm que satisfazer as equações de autovalores e as condições de contorno para qualquer instante $t$, o que as força a ser dependentes do tempo. Assim, as autofunções de nosso problema podem ser escritas como:

$$
\varphi_{n}(t, x)=\sqrt{\frac{2}{l(t)}} \operatorname{sen}\left[\omega_{n}(t) x\right],
$$

com freqüência $\omega_{n}(t)=\frac{n \pi}{l(t)}$ e que satisfazem às condições de ortonormalidade e completeza requeridas. Isso nos permite expandir o campo escalar nessas autofunções introduzindo as funções de modo (variáveis canônicas) $q_{n}(t)$ : 


$$
\phi(t, x)=\sum_{n} q_{n}(t) \varphi_{n}(t, x)
$$

Inserindo essa expansão em (6.68), multiplicando por $\varphi_{m}$ e utilizando as condições de ortonormalidade e completeza, podemos integrar no intervalo $I(t)$ para obtermos as equações de movimento para $q_{n}$ :

$$
\ddot{q}_{n}+\Omega_{n}^{2}(t) q_{n}-2 \sum_{m} M_{n m}(t) \dot{q}_{m}+\sum_{m}\left[\dot{M}_{m n}(t)-N_{n m}(t)\right] q_{m}=0
$$

em que $\Omega_{n}^{2}(t)=\omega_{n}^{2}(t)+m^{2}$ e $M_{n m}(t)$ e $N_{n m}(t)$ são as matrizes de acoplamento definidas como:

$$
\begin{aligned}
& M_{n m}(t)=\int_{I(t)} d x \dot{\varphi}_{n}(t, x) \varphi_{m}(t, x)=\left\{\begin{array}{l}
\frac{i(t)}{l(t)}(-1)^{n+m} \frac{2 n m}{m^{2}-n^{2}} \text { se } n \neq m \\
0 \text { se } n=m
\end{array}\right. \\
& N_{n m}(t)=\int_{I(t)} d x \dot{\varphi}_{n}(t, x) \dot{\varphi}_{m}(t, x)=\sum_{k} M_{n k} M_{m k} .
\end{aligned}
$$

Finalmente podemos ver pela equação de movimento (6.72) a principal característica de problemas em que efeitos de borda importam: que a evolução temporal dos modos do campo é descrita por um sistema de equações diferenciais acopladas, que, como pudemos ver, são conseqüência direta da dependência temporal das placas e das condições de contorno que foram impostas ao problema.

Isso leva a outra característica central desse tipo de sistema em que um mecanismo extra de criação de partículas aparece vindo do acoplamento dos modos dependente do tempo, dado por $M_{n m}(t)$. Esse efeito é muitas vezes chamado de efeito de aceleração. Assim, a dependência temporal da freqüência $\omega_{n}(t)$ e de $M_{n m}(t)$ corresponde a duas fontes de criação de partículas das flutuações do vácuo. A fonte correspondente à dependência temporal da freqüência é igual a fonte de criação de partículas que tratamos anteriormente, devido ao squeezing do vácuo, no efeito Schwinger.

Podemos ver também que a solução depende fortemente da forma do movimento $l(t)$. A estrutura do acoplamento entre os modos, mediada pela matriz $M_{n m}(t)$, depende do tipo de condição de contorno utilizada. No nosso caso, como utilizamos condições de contorno de Dirichlet, $M_{n m}$ é antissimétrica, assim $M_{m n}-M_{n m}=-2 M_{n m}$.

Como vimos acima, esse simples modelo de um campo escalar em $1+1$ dimensões já nos fornece as características mais importantes encontradas no ECD.

Dessa forma, podemos generalizar o problema para um caso mais realístico: o de uma 
cavidade tridimensional no vácuo eletromagnético. O estudo desse problema é importante, pois é a base da descrição teórica do experimento que pretende verificar o ECD, que vamos mencionar na Seção 6.3.3.

\subsubsection{O campo eletromagnético em uma cavidade dinâmica}

Queremos descrever as condições de contorno do campo eletromagnético em uma cavidade retangular com paredes perfeitamente refletoras (espelhos ideais), que dão origem a dois modos distintos de polarização: o modo transverso elétrico e o transverso magnético. Assim, o problema da dinâmica do campo eletromagnético na cavidade pode ser dividido em dois problemas de contorno separados para cada uma dessas polarizações.

Vamos partir das equações de Maxwell descrevendo o campo eletromagnético livre $[155,156]$ :

$$
\begin{gathered}
\nabla \mathbf{E}(t, \mathbf{x})=0, \nabla \mathbf{B}(t, \mathbf{x})=0 \\
\nabla \wedge \mathbf{E}(t, \mathbf{x})=-\partial_{t} \mathbf{B}(t, \mathbf{x}), \nabla \wedge \mathbf{B}(t, \mathbf{x})=\partial_{t} \mathbf{E}(t, \mathbf{x}) .
\end{gathered}
$$

Levando em conta, agora, que as paredes são perfeitamente condutoras e planas (espelhos ideais), com vetor normal $\hat{\mathbf{x}}$ apontando na direção $\mathrm{x}$, isso impõe condições de contorno no campo eletromagnético no espelho do tipo:

$$
\begin{aligned}
\mathbf{E}_{\|} & =0 \Leftrightarrow \hat{\mathbf{x}} \wedge \mathbf{E}=0 \\
\mathbf{B}_{\perp} & =0 \Leftrightarrow \hat{\mathbf{x}} \cdot \mathbf{B}=0,
\end{aligned}
$$

em que $\|$ e $\perp$ denotam os campos nas direções paralela e perpendicular ao espelho, respectivamente.

Dadas essa condições, se considerarmos agora um dos espelhos em movimento, na direção $x$, essas condições devem ser impostas no referencial comóvel de Lorentz, no qual o espelho está instantaneamente em repouso. O desenvolvimento feito usualmente nesses casos é decompor o campo elétrico e magnético nos modos transverso elétrico (TE) e transverso magnético $(\mathrm{TM}), \operatorname{com} \mathbf{E}^{(T E)}(t, \mathbf{x}) \cdot \hat{\mathbf{x}}=0$ e $\mathbf{B}^{(T M)}(t, \mathbf{x}) \cdot \hat{\mathbf{x}}=0$. Com isso, podemos definir também potenciais vetores $\mathbf{A}^{(T E)}$ e $\mathbf{A}^{(T M)}$ que dão origem a esses campos. Esses potenciais estão sujeitos ao calibre de Coulomb e satisfazem a equação de onda:

$$
\nabla \cdot \mathbf{A}^{(T E / T M)}=0, \square \mathbf{A}^{(T E / T M)}(t, \mathbf{x})=0
$$

em que $\mathbf{A}^{(T E)}(t, \mathbf{x}) \cdot \hat{\mathbf{x}}=0$ e $\mathbf{A}^{(T M)}(t, \mathbf{x}) \cdot \hat{\mathbf{x}}=0$. 
Como afirmamos acima, as condições de contorno devem ser impostas no referencial comóvel de Lorentz. Ou seja, no referencial do laboratório, $S\left(t_{0}\right)$, a posição do espelho móvel é dada por $x_{\text {espelho }}=l(t)$, em que para tempos muito próximos de $t_{0}$, a posição do espelho é considerada como sendo $x_{\text {espelho }}=\dot{l}\left(t_{0}\right)\left(t-t_{0}\right)+l\left(t_{0}\right)$, em que $l\left(t_{0}\right)$ é a posição do espelho em $t_{0}$. Logo, o referencial comóvel de Lorentz, $S^{\prime}\left(t_{0}\right)$, no qual as condições de contorno devem ser impostas, está relacionado com $S\left(t_{0}\right)$ via transformações de Lorentz.

Em (6.76) e (6.77) temos as condições de contorno dos campos elétrico e magnético no referencial $S^{\prime}\left(t_{0}\right)$. Isso corresponde, devido à definição do potencial vetor, às condições de contorno para os potenciais:

$$
\begin{gathered}
\partial_{t^{\prime}} \mathbf{A}^{(T E)}\left(t^{\prime}=0, x^{\prime}=0, \mathbf{x}_{\|}^{\prime}\right)=0 \\
\partial_{x^{\prime}} \mathbf{A}^{(T M)}\left(t^{\prime}=0, x^{\prime}=0, \mathbf{x}_{\|}^{\prime}\right)=0
\end{gathered}
$$

em que as coordenadas com ', representam o referencial comóvel. Fazendo uma transformação de Lorentz para passarmos para o referencial do laboratório: $\partial_{t^{\prime}}=\gamma\left(v \partial_{x}+\partial_{t}\right)=$ $d / d t$ e $\partial_{x^{\prime}}=\gamma\left(\partial_{x}+v \partial_{t}\right), \operatorname{com} \gamma=1 / \sqrt{1-v^{2}}$, temos que as condições de contorno:

$$
\begin{aligned}
\mathbf{A}^{(T E)}(t, x & \left.=l(t), \mathbf{x}_{\|}\right)=0, \\
\left(\partial_{x}+v \partial_{t}\right) \mathbf{A}^{(T M)}(t, x & \left.=l(t), \mathbf{x}_{\|}\right)=0,
\end{aligned}
$$

sendo que no modo TE, como a derivada total era igual a zero, então o potencial será igual a uma constante, que pode ser igual a zero, sem perda de generalidade.

Aqui, chegamos a um importante resultado. Como pudemos ver acima, os modos TE são sujeitos a condições de contorno de Dirichlet e os modos TM estão sujeitos a condições de contorno de Neumann generalizada, ou seja, condições de contorno de Neumann e derivada temporal.

Com isso em mãos, consideremos agora a cavidade retangular feita de paredes perfeitamente condutoras com dimensões $\left(l_{x}=l(t), l_{y}, l_{z}\right)$, Figura 6.3.2. Podemos observar das condições de contorno derivadas acima que o modo TE pode ser relacionado com um campo escalar sujeito a condições de contorno de Dirichlet. Analogamente, isso pode ser feito para o modo TM e as condições de contorno de Neumann generalizadas. Com isso, podemos descrever a dinâmica de um campo eletromagnético em uma cavidade retangular como um problema de um campo escalar com condições de contorno separadas para as polarizações: 


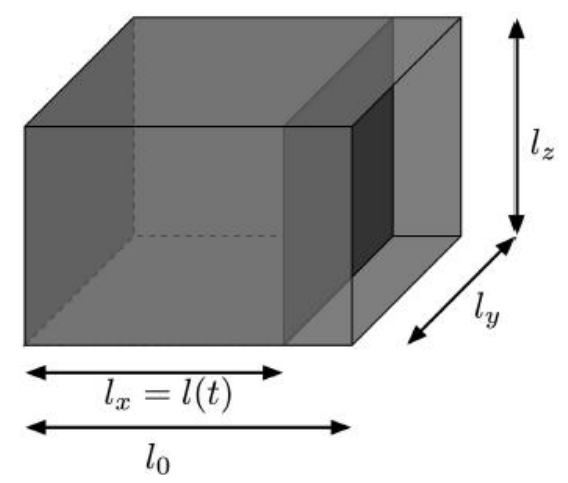

Figura 6.6: Cavidade tridimensional com paredes perfeitamente condutoras, em que uma parede se move na direção $x$, com trajetória $l(t)$. Figura retirada de [82].

$$
\square \phi(t, \mathbf{x})=0, \text { com }\left\{\begin{array}{l}
\left.\phi\right|_{\text {paredes }}=0, \text { modo TE } \\
\left.\left(\partial_{x}+v \partial_{t}\right) \phi\right|_{x=l(t)}=\left.\partial_{x} \phi\right|_{\text {paredes estticas }}, \text { modo TM }
\end{array} .\right.
$$

Essa simplificação do problema permite-nos estudar os modos de maneira separada. Convém mencionar que as taxas de criação para cada um desses modos é correspondente à taxa para cada uma das condições de contorno.

\subsubsection{Modo transverso magnético}

A evolução dinâmica dos modos TM é a mesma de um campo escalar sujeito a condições de contorno de Neumann [156]. Assim, o problema é descrito pela equação de onda (6.83), sujeita a condições de contorno de Neumann em todas as paredes da cavidade. Como feito no caso unidimensional (6.71), expandimos esse campo escalar nas variáveis canônicas, usando o análogo tridimensional da base instantânea (6.70), para $t>0$ :

$$
\varphi_{\mathbf{n}}(t, x)=\frac{1}{2 \Omega_{\mathbf{n}}^{(3)}(t)} \sqrt{\frac{2}{l_{x}}} \cos \left[\frac{n_{x} \pi}{l_{x}} x\right] \sqrt{\frac{2}{l_{y}}} \cos \left[\frac{n_{y} \pi}{l_{y}} y\right] \sqrt{\frac{2}{l_{z}}} \cos \left[\frac{n_{z} \pi}{l_{z}} z\right],
$$

e para $t \leq 0$, representa a cavidade estática:

$$
\varphi_{\mathbf{n}}(t, x)=\frac{1}{2 \Omega_{\mathbf{k}}^{(3)}} \sqrt{\frac{2}{l(t)}} \cos \left[\frac{k_{x} \pi}{l(t)} x\right] \sqrt{\frac{2}{l_{y}}} \cos \left[\frac{k_{y} \pi}{l_{y}} y\right] \sqrt{\frac{2}{l_{z}}} \cos \left[\frac{k_{z} \pi}{l_{z}} z\right],
$$


em que $\left(k_{x}, k_{y}, k_{z}\right)>0$ e $\Omega_{\mathbf{k}}^{(3)}$ é a freqüência para o caso estático. Essa base instantânea satisfaz as condições de contorno de Neumann e forma um conjunto ortonormal e completo de autofunções do laplaciano tridimensional, $-\triangle_{(3)}$, com autovalores dependentes do tempo:

$$
\Omega_{\mathbf{n}}^{(3)}(t)=\sqrt{\left(\frac{\pi n_{x}}{l(t)}\right)^{2}+\underbrace{\left(\frac{\pi n_{y}}{l_{y}}\right)^{2}+\left(\frac{\pi n_{y}}{l_{y}}\right)^{2}}_{k_{\|}^{2}}} .
$$

Cada modo $\mathbf{n}$ é rotulado por três valores $n_{x}, n_{y}, n_{z}=1,2, \ldots$, em que usamos a notação $\mathbf{n}=\left(n_{x}, n_{y}, n_{z}\right)$. Inserindo a expansão na equação de onda, multiplicando por $\varphi_{\mathbf{m}}(t, \mathbf{x})$ e integrando na dimensões espaciais, como feito anteriormente, chegamos a uma equação de movimento para as variáveis canônicas:

$$
\begin{aligned}
\ddot{q}_{\mathbf{n}}+\Omega_{\mathbf{n}}^{(3) 2}(t) q_{\mathbf{n}} & =-2 \lambda(t) \sum_{\mathbf{m}} M_{\mathbf{n m}}(t) \dot{q}_{\mathbf{m}}-\dot{\lambda}(t) \sum_{\mathbf{m}} M_{\mathbf{n m}} q_{\mathbf{m}} \\
& -2 \dot{\lambda}(t) l^{2}(t) \sum_{\mathbf{m}} N_{\mathbf{n m}}(t) \ddot{q}_{\mathbf{m}}-\sum_{\mathbf{m}}\left[N_{\mathbf{n m}} \ddot{\lambda}(t) l^{2}(t)-\lambda(t) Q_{\mathbf{n m}}\right] \dot{q}_{\mathbf{m}} \\
& -\lambda(t) l^{2}(t) \sum_{\mathbf{m}} N_{\mathbf{n m}} \partial_{t} \ddot{q}_{\mathbf{m}}
\end{aligned}
$$

em que os coeficientes de acoplamento são dados por $[82,156]$ :

$$
\begin{aligned}
M_{\mathbf{n m}} & =l(t) \int_{0}^{l(t)} d x \int_{0}^{l_{y}} d y \int_{0}^{l_{z}} d z \frac{\partial \phi_{\mathbf{n}}(t, \mathbf{x})}{\partial l(t)} \phi_{\mathbf{m}}(t, \mathbf{x}) \\
& =\left\{\begin{array}{l}
(-1)^{n_{x}+m_{x}} \frac{2 n_{x} m_{x}}{m_{x}^{2}-n_{x}^{2}} \delta_{n_{y} m_{y}} \delta_{n_{z} m_{z}}, \text { se } n_{x} \neq m_{x}, \\
-\delta_{n_{y} m_{y}} \delta_{n_{z} m_{z}}, \text { se } n_{x}=m_{x},
\end{array}\right. \\
\lambda(t)= & i(t) / l(t), \\
N_{\mathbf{n m}}= & \int_{0}^{l(t)} d x \int_{0}^{l_{y}} d y \int_{0}^{l_{z}} d z v \phi_{\mathbf{n}}(t, \mathbf{x}) \phi_{\mathbf{m}}(t, \mathbf{x}), \\
Q_{\mathbf{n m}}= & l^{2}(t) \int_{0}^{l(t)} d x \int_{0}^{l_{y}} d y \int_{0}^{l_{z}} d z\left[\left(v^{\prime \prime}-\Omega_{\mathbf{m}}^{(3) 2} v\right) \phi_{\mathbf{n}}(t, \mathbf{x}) \phi_{\mathbf{m}}(t, \mathbf{x})+2 v^{\prime} \phi_{\mathbf{n}}^{\prime}(t, \mathbf{x}) \phi_{\mathbf{m}}(t, \mathbf{x})\right],
\end{aligned}
$$

em que a derivada " ' " é a derivação em relação a x.

Se tivéssemos utilizado o modo TE, ou seja, condições de Dirichlet, a equação (6.87) 
exibiria o mesmo tipo de acoplamento que o caso anterior unidimensional [156, 82].

Contudo, utilizamos o modo TM, uma vez que em todos os modos TE temos $n_{x}=0$. A utilização do modo TM é a única maneira de obtermos o primeiro modo excitado pela freqüência. Em cavidades com $l_{x} \ll l_{y}, l_{z}$ somente modos TM podem ser excitados.

Com esses resultados em mão, podemos calcular o número de partículas (fótons) criados. Vamos discutir aqui alguns resultados analíticos conhecidos.

\section{Número de fótons criados}

Para podermos calcular esse número de partículas, são utilizadas as transformadas de Bogoliubov, fazendo um procedimento análogo ao feito na Seção 5.2.2. Primeiramente consideramos dois instantes, $t_{\text {in }}$ e $t_{\text {out }}$, em que a fronteira $l(t)$ está em repouso para $t<t_{\text {in }}$ e $t>t_{\text {out }}$, que inclui os tempo assintóticos, $t_{\text {in }} \rightarrow-\infty$ e $t_{\text {out }} \rightarrow+\infty$, assim como os instantes finitos em que o movimento ainda não havia começado ou terminado. Com isso, a configuração inicial (in) e final (out) do sistema é dada por:

$$
\begin{gathered}
\text { in }: \Omega_{n}^{\text {in }}=\Omega_{n}\left(t<t_{\text {in }}\right)=\text { const } \neq 0, \quad M_{n m}\left(t<t_{\text {in }}\right)=0, \\
\text { out }: \Omega_{n}^{\text {out }}=\Omega_{n}\left(t>t_{\text {out }}\right)=\text { const } \neq 0, M_{\text {nm }}\left(t>t_{\text {out }}\right)=0,
\end{gathered}
$$

em que, como feito anteriormente, a configuração inicial não tem que ser igual à final. Podemos escrever, como feito para o campo na Seção 5.2.2, as variáveis canônicas em função dos operadores de criação e aniquilação no estado in e, separadamente, no estado out. Esses operadores, de aniquilação, definem o vácuo inicial e final. A transformação canônica que relaciona esses operadores in e out é a transformada de Bogoliubov, que nos permite escrever o número de partículas criadas da forma (5.94), como já obtida anteriormente na Seção 5.2.2, para o caso em que o estado inicial é vácuo. Para a criação estimulada, no ECD, vemos que a fórmula (5.94) é válida, faltando apenas um termo vindo do acoplamento dos modos.

O caso em que temos soluções analíticas é para um movimento oscilatório da parede:

$$
l(t)=l_{0}\left[1+\epsilon \operatorname{sen}\left(\omega_{\text {cav }} t\right)\right], \epsilon \ll 1 .
$$

Vamos estudar o caso em que o número de partículas cresce exponencialmente: o caso da ressonância paramétrica, $\omega_{\text {cav }}=2 \Omega_{n}^{i n}$. Esse caso é de interesse, uma vez que é a melhor chance de se observar o ECD, pois representa a maior taxa de criação de partículas. Podemos obter o número de partículas introduzindo os parâmetros de squeezing, como feito na Seção 5.2.2.4, com parametrização invertida em relação à feita na Seção 5.2.2.4. Logo, o número médio de fótons no modo TM criados, ou para a criação de partículas escalares com condições de Neumann, para os modos não-desacoplados k, é dado por 
[155]:

$$
N_{n}(t)=\sinh ^{2}\left(\gamma_{n} \epsilon t\right), \operatorname{com} \gamma_{n}=\frac{n}{\Omega_{n}^{i n}}\left(\frac{\pi}{l_{0}}\right)^{2} .
$$

Esse é o resultado mais expressivo do ECD: devido a esse crescimento exponencial do número de partículas devido à ressonância paramétrica, é possível ter um número de partículas criadas suficientes para serem detectadas. Outros cálculos do ECD em uma dimensão [147] obtiveram um valor para o número médio de partículas criadas que crescia linearmenteno tempo. Esse resultado deriva-se do cálculo de um modelo simplificado, em que a condição das paredes são perfeitamente condutoras. Como os autores mesmo mencionam, esse modelo simplificado perde muitos fenômenos físicos importantes.

Podemos ver que o número de partículas criadas pelos modos TM é muitas vezes maior que as criadas pelos modos TE. Dessa forma, utilizamos o modo TM para a determinação da criação de partículas.

\subsubsection{Experimentos}

O ECD é o fenômeno de criação de partículas que apresenta propostas experimentais de sua verificação viáveis para um futuro próximo. Dessa forma, representa uma esperança para a verificação experimental da criação de partículas dinâmica, o que validaria também o ESD, uma vez que a analogia entre os efeitos é clara.

O conhecido efeito Casimir foi verificado experimentalmente em 1997 por Steve K. Lamoreaux do Laboratório Nacional de Los Alamos [157, 158] e por Umar Mohideen da Universidade da Califórnia e seu colega Anushree Roy [159], em que as dificuldades experimentais de se realizar um arranjo experimental de duas placas paralelas foi vencido utilizando um sistema de uma placa e um esfera, para garantir o paralelismo.

Contudo, o ECD, no qual uma das placas é acelerada de forma não-uniforme, ou de forma mais conhecida, quando a superfície é uma superfície metálica e passa a oscilar, ainda não foi experimentalmente observado. Isso se deve ao fato de o conceito de oscilações mecânicas de uma das placas ou da parede de uma cavidade apresentar um impedimento prático. Com a tecnologia atual podemos ver que é praticamente impossível obter-se um experimento com uma cavidade com uma parede móvel. As maiores freqüências atingidas atualmente para um movimento mecânico estão na faixa dos giga hertz e seguindo a necessidade do efeito Casimir dinâmico de amplificação paramétrica, isso implica em cavidades em microondas com dimensões na faixa de $1 \mathrm{~cm}$ a $1 \mathrm{~m}$. Temos ainda que o movimento mecânico de uma das paredes dessa cavidade requer uma quantidade muito grande de energia. Por exemplo, se a parede de um cavidade feita de um material com densidade de massa $\rho=3 \times 10^{3} \mathrm{~kg} / \mathrm{m}^{3}$, oscilar com freqüência angular $\omega$, tal que $\omega / 2 \pi=2 G H z$, e amplitude de oscilação $\delta x=1 \mathrm{~nm}$, para uma parede com volume $V=3 \mathrm{~cm} \times 3 \mathrm{~cm} \times 0,1 \mathrm{~mm}$ (valores esses que, pela teoria do ECD, nos forneceriam uma 
quantidade de fótons detectável), seria necessária um potência de $3 \times 10^{8} \mathrm{~W}$ para mover mecanicamente essa parede [160].

Recentemente, Kim et al [161], propuseram um experimento para detecção indireta do ECD, em que movimento mecânico é empregado. Com o uso da tecnologia de filmes finos, os autores afirmam que é possível um movimento mecânico na ordem de giga hertz e que, com isso, teriam uma produção de fótons de Casimir que, apesar de pequena, seria passível da deteç̧ão indireta através da interação com átomos de metais alcalinos ultra frios.

Esse ainda é só um experimento mental, apesar dos autores afirmarem que é acessível com a presente tecnologia. Contudo, experimentos baseados em movimento mecânicos das paredes ainda não foram realizados. Com isso, vamos expor o experimento MIR, que apresenta uma alternativa para as oscilações mecânicas da parede substituídas por um movimento induzido, com o uso de um supercondutor. Esse experimento já está montado e em fase final de testes, como vamos ver abaixo.

\subsubsection{MIR: Motion Induced Radiation}

Reconhecendo as limitações experimentais para as oscilações mecânicas da parede, foi proposto o experimento MIR (Motion Induced Radiation) [127, 166]. Esse experimento é conduzido pelo grupo italiano de Dino Zanello (Rome), Caterina Braggio e Gianni Carugno (Padova), Giuseppe Messineo e Federico Della Vallee (Trieste), Giacomo Bressi, Antonio Agnesi, Federico Pirzio, Alessandra Tomaselli e Giancarlo Reali (Pavia), Giuseppe Galeazzi e Giuseppe Ruoso (Legnaro Labs); e sua descrição teórica foi feita principalmente por V.V. Dodonov et al. [162]

O experimento consiste em usar o movimento efetivo de uma parede de uma cavidade ressonante supercondutora, baseada nos trabalhos de Yablonovitch (1989) [163] e Lozovik (1995) [164, 165]. Esse movimento efetivo é produzido pela incidência periódica na placa supercondutora por um laser com freqüência com amplitude modulada ultra-alto. A ação do laser no semicondutor faz com que suas características mudem de completamente transparente para completamente refletivo. Os pulsos de laser iluminam o semicondutor com uma freqüência de repetição igual a duas vezes a freqüência de ressonância de cavidade, que é a condição de ressonância paramétrica necessária para que a quantidade de fótons produzida seja detectável. Esse método está esquematizado na Figura 6.7. 


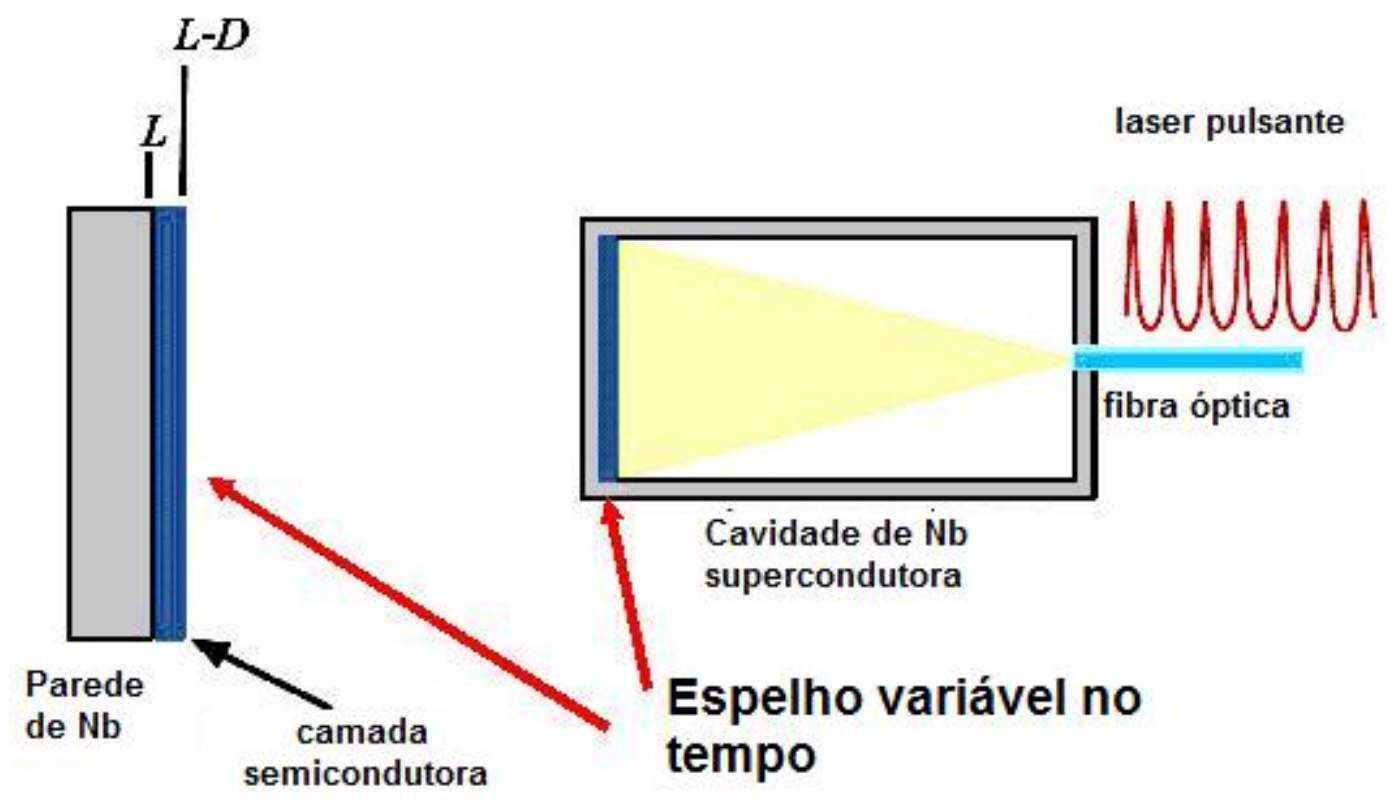

Figura 6.7: Esquema do experimento MIR, em que podemos ver o mecanismo que produz o movimento da parede, em que o supercondutor, devido ao laser pulsante incidente, muda suas propriedades de totalmente refletor para totalmente transparente [127].

O arranjo experimental é dividido em sistema de laser, cavidade ressonante com semicondutor, câmara de recepção e aquisição de dados. Podemos ver pela Figura 6.8, que o esquema experimental da cavidade consiste em uma cavidade de niobium de dimensões $9 \times 1 \times 8 \mathrm{~cm}^{3}$ a temperaturas criogênicas colocada no vácuo. O semicondutor é dado por uma camada de GaAs em uma parede da cavidade de $\mathrm{Nb}$. O GaAs foi escolhido porque apresentava as melhores características necessárias para o experimento, como tempo de recombinação e mobilidade. O laser, trazido na fibra óptica, é ajustado próximo ao infravermelho e modulado em amplitude a uma freqüência exatamente igual ao dobro da freqüência de ressonância. A energia total armazenada no laser é limitada, com isso o número de pulsos disponíveis também o será e está estimada em $10^{3}$ a $10^{4}$ pulsos em cada tomada de dados. Todos os componentes estão colocados em um criostato de hélio líquido que mantém a temperatura de 1 a $8 \mathrm{~K}$. Assim, quando a camada semicondutora está totalmente refletora, ela se comporta como um espelho; quando ela está totalmente transparente, a parede de niobium é que faz o papel de espelho, dando a impressão de que o espelho foi deslocado. 


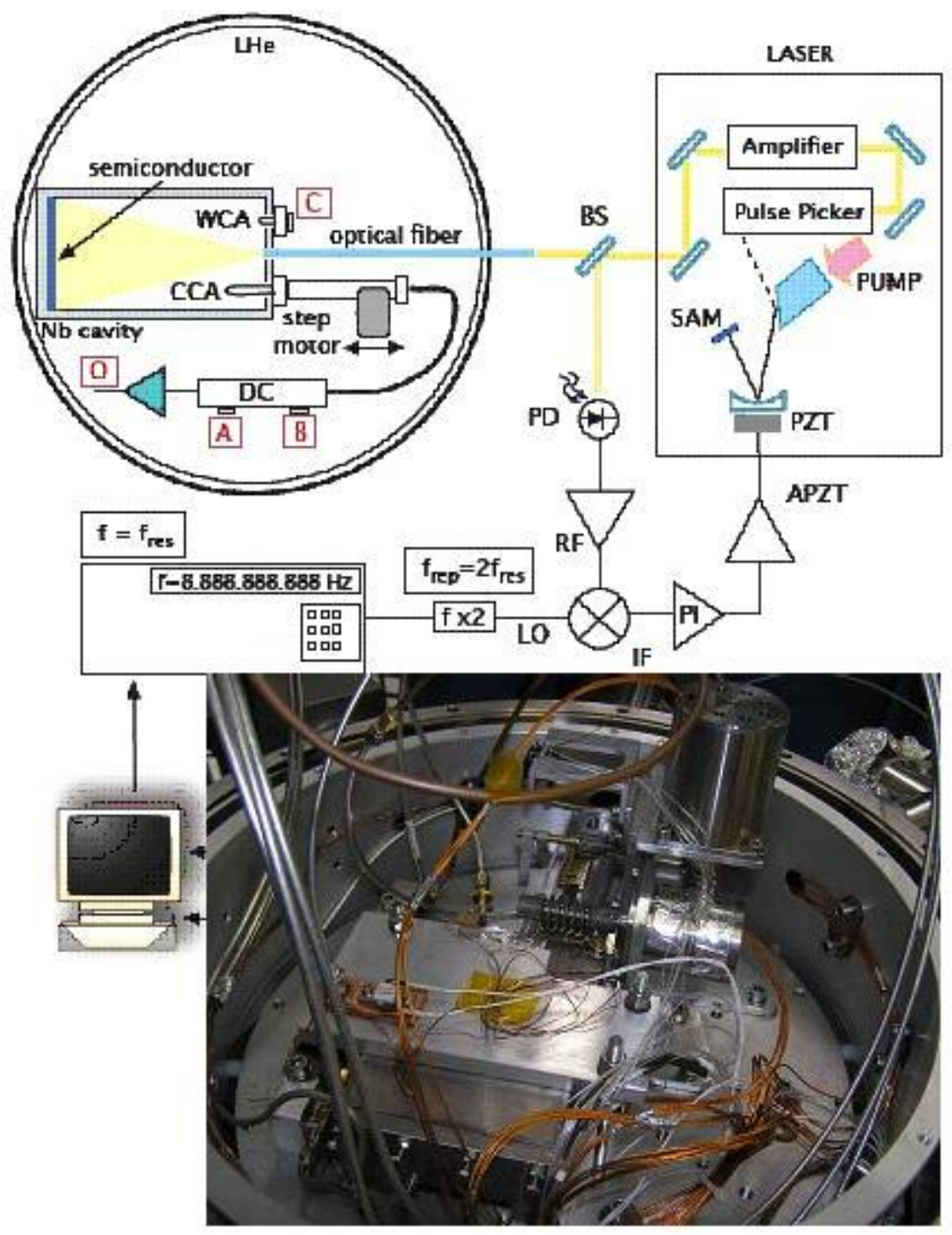

Figura 6.8: Arranjo experimental do experimento MIR [127].

Convém salientar que esse arranjo experimental permite uma grande variação dos parâmetros experimentais como a temperatura, podendo assim verificar possíveis efeitos térmicos e alterações nas previsões do efeito Casimir dinâmico a temperaturas mais altas; a freqüência do laser e, conseqüentemente o movimento efetivo do espelho, verificando assim o comportamento para freqüências diferentes da freqüência de ressonância paramétrica; e diferentes materiais e grossuras do espelho.

Alguns problemas experimentais ainda estão sendo resolvidos. Os referentes ao laser, ao semicondutor e à detecção são os principais. Podemos citar principalmente este último, sendo necessário serem determinados qual o mínimo sinal (número de partículas) que é possível ser detectado e um ruído da radiação de corpo negro. Algumas medidas foram feitas e estima-se que, considerando esses efeitos, a sensibilidade de detecção é de aproximadamente 100 fótons. 
Sabendo que, se fizermos uma estimativa da quantidade de fótons que será possível detectar no experimento, utilizando parâmetros realísticos experimentalmente e as estimativas teóricas feitas por V. V. Dodonov et al. (2006) [162], a estimativa é de $N>10^{3}$ por pulsos do laser. Com isso, vemos que a sensibilidade do experimento é muito boa.

No ano de 2008, os ajustes finais do experimento ainda estavam sendo feitos, bem como suas calibrações. O grupo responsável acreditava que resultados preliminares seriam medidos no final de 2008, assim uma estimativa mais que otimista para a comunidade científica é de resultados para 2009. 


\section{Capítulo 7}

\section{Análogos Cosmológicos}

Introduzimos nos capítulos anteriores a teoria da inflação que resolve os problemas do MCP e também nos fornece uma explicação para a criação das pertubações iniciais responsáveis pela formação das estruturas em larga escala de nosso universo. Para isso a teoria da inflação combina a relatividade geral e a teoria quântica, sem esbarrar no problema da necessidade de uma teoria da gravitação quântica. Para que seja possível o aparecimento dessas perturbações primordiais, dada pela criação de partículas, um efeito muito importante, não-trivial e não-perturbativo do vácuo ocorre que é a criação de partículas do vácuo devido à expansão do universo. Vimos também que essa criação de partículas ocorre no pré-aquecimento, devido à oscilação do campo do inflaton em torno do mínimo de seu potencial.

Vamos mostrar nesse capítulo que esses efeitos quânticos do universo primordial são equivalentes aos já descritos nos capítulos anteriores efeitos Schwinger estático e oscilatório. Ou seja, mostraremos que esses mecanismos do universo primordial equivalem à campos quânticos interagindo com campos externos clássicos que no caso do efeito Schwinger é eletromagnético e no caso cosmológico é gravitacional, somente mudando a origem do campo externo aplicado.

Essa analogia é de grande importância, uma vez que a verificação experimental em Terra dos efeitos de criação de partículas eletromagnéticos evidenciaria a possibilidade de existência do mecanismo cosmológico equivalente, o que é relevante uma vez que esses mecanismos inflacionários de criação de partículas são cruciais para o entendimento moderno sobre a origem e a evolução do universo e ainda permanecem como previsões puramente teóricas. Como vimos nos Capítulos 5 e 6 esses efeitos estão próximos de serem detectados experimentalmente.

Vamos primeiramente mostrar a equivalência entre os mecanismo de criação de partículas estáticos, mostrando que é possível escrevermos o processo inflacionário com equações análogas ao efeito Schwinger estático. Em seguida passaremos para o efeito dinâmico, em que mostraremos que as equações de criação de partículas do pré-aquecimento naturalmente apresentam a mesma forma do efeito Schwinger oscilatório. 
Vamos trabalhar nesse capítulo com o observável número de partículas criadas (ou densidade de número de partículas criadas) como feito nos capítulos do efeito Schwinger ao invés dos observáveis usuais de cosmologia, o espectro de potências ou função de correlação de dois pontos, que são utilizados no contexto cosmológico, uma vez que são relacionados com sua possibilidade de observação. Contudo, para o estudo dos mecanismos de criação de partículas, o observável a ser utilizado deve ser o número de partículas criadas, uma vez que essa criação ocorre fora do equilíbrio térmico e esse observável é um invariante adiabático.

\subsection{Criação de Partículas na Inflação e o Efeito Schwin- ger Estático}

O efeito Schwinger estático consiste em um processo não-perturbativo de perturbação do vácuo quântico com um campo externo elétrico constante muito forte que resulta na criação de pares de partículas do vácuo. Esse efeito foi modelado pela TQC, no Capítulo 5, como um campo fermiônico ou escalar complexo interagindo com esse potencial externo.

Já na teoria da inflação, como vimos, para que haja a criação de partículas, ou uma perturbação na matéria, é necessário que a métrica seja perturbada e essa perturbação ocorre devido à expansão do universo. Esse fundo dinâmico, que é o responsável pela criação de partículas deve ser muito forte para que o mecanismo de criação seja eficiente. No universo primordial temos que o campo gravitacional é muito forte, da ordem de $H / m_{p l} \sim 10^{-5}$. Utilizamos como campo quântico ao invés da perturbação da métrica as variáveis de Mukhanov-Sasaki, definidas no Capítulo 4, que no caso das perturbações escalares é uma combinação do potencial de Bardeen, que descreve o potencial newtoniano generalizado para a relatividade geral, e as perturbações do campo do inflaton. Dessa forma, a criação de partículas na inflação corresponde à quantização de um campo escalar real, descrito pela variáveis de Mukhanov-Sasaki, em um espaço-tempo curvo.

A primeira vista temos dois mecanismos de criação de partículas distintos, um nãoperturbativo e outro perturbativo. Vamos mostrar então que a criação de partículas no período inflacionário, ou seja, um campo escalar em um espaço-tempo curvo, é equivalente a um campo escalar na presença de um potencial externo, que no caso é gravitacional, e que essa correspondência é feita a menos de uma transformação canônica [84, 86].

A forma requerida para ser análoga ao efeito Schwinger foi obtida na Seção 4.2 do Capítulo 4. Contudo, a Lagrangeana quadrática foi obtida através de um método perturbativo até segunda ordem. O que vamos mostrar aqui é um argumento mais forte e não-perturbativo: que o campo escalar livre em um espaço-tempo curvo, representado na inflação, pode ser escrito, sem aproximações ou cálculo perturbativos, como um campo escalar em presença de um potencial externo. Vamos mostrar isso para o caso geral de 
um campo escalar qualquer que será aplicado ao caso inflacionário posteriormente.

\subsubsection{Campo Escalar Livre em um Espaço-Tempo Curvo}

Vamos quantizar um campo escalar livre em um espaço-tempo curvo, dado pelo funcional de ação [84]:

$$
S[\Phi]=-\frac{1}{2} \int d^{4} x \sqrt{-g} g^{\mu \nu} \partial_{\mu} \Phi \partial_{\nu} \Phi .
$$

Essa ação é da forma da ação de Einstein-Hilbert (4.82) que encontramos na inflação. Se considerarmos a métrica de FRW, $d s^{2}=a^{2}(\eta)\left(d \eta^{2}-\delta_{i j} d x^{i} d x^{j}\right)$, temos que a ação fica na forma:

$$
S[\Phi]=-\frac{1}{2} \int d^{4} x a^{2}(\eta)\left[\Phi^{\prime 2}-\delta^{i j} \partial_{i} \Phi \partial_{j} \Phi\right] .
$$

Vamos fazer uma transformada de Fourier do campo escalar para podermos estudar modo a modo o problema. No caso vamos reescalonar os modos de Fourier por conveniência por um fator de $a^{-1}(\eta)$, obtendo:

$$
\Phi(\eta, \mathbf{x})=\frac{1}{a(\eta)} \int \frac{d^{3} k}{(2 \pi)^{3 / 2}} \mu_{\mathbf{k}}(\eta) \mathrm{e}^{i \mathbf{k x}}
$$

em que como o campo escalar é real, $\mu_{\mathbf{k}}^{*}=\mu_{-\mathbf{k}}$. Substituindo essa expansão em (7.2), obtemos a ação para os componentes complexos de Fourier, separadas as variáveis reais e as imaginárias da forma $\mu_{\mathbf{k}}=\frac{1}{\sqrt{2}}\left(\mu_{\mathbf{k}}^{R}+i \mu_{\mathbf{k}}^{I}\right)$ :

$$
\begin{aligned}
S & =\frac{1}{2} \int d \eta \int_{\mathbb{R}_{+}^{3}} d^{3} k\left\{\left(\mu_{\mathbf{k}}^{R^{\prime}}\right)^{2}+\left(\mu_{\mathbf{k}}^{I^{\prime}}\right)^{2}-2 \frac{a^{\prime}}{a}\left(\mu_{\mathbf{k}}^{R} \mu_{\mathbf{k}}^{R}+\mu_{\mathbf{k}}^{I} \mu_{\mathbf{k}}^{I^{\prime}}\right)\right. \\
& \left.+\left(\frac{a^{\prime 2}}{a^{2}}-k^{2}\right)\left[\left(\mu_{\mathbf{k}}^{R}\right)^{2}+\left(\mu_{\mathbf{k}}^{I}\right)^{2}\right]\right\} .
\end{aligned}
$$

Temos então uma ação separada para cada $\mu_{\mathbf{k}}^{R, I}$.

Vamos passar agora para o formalismo Hamiltoniano. Primeiramente, vamos definir o momento canonicamente conjugado a $\mu_{\mathbf{k}}^{R, I}$ :

$$
p_{\mathbf{k}}^{R, I}=\frac{\partial \mathcal{L}}{\partial \mu_{\mathbf{k}}^{R, I^{\prime}}}=\mu_{\mathbf{k}}^{R, I^{\prime}}-\frac{a^{\prime}}{a} \mu_{\mathbf{k}}^{R, I} .
$$

Fazendo uma transformação de Legendre, podemos escrever a Hamiltoniana do problema: 


$$
H=\int_{\mathbb{R}_{+}^{3}} d^{3} k\left\{\frac{1}{2}\left[\left(p_{\mathbf{k}}^{R}\right)^{2}+2 \frac{a^{\prime}}{a} \mu_{\mathbf{k}}^{R} p_{\mathbf{k}}^{R}+k^{2}\left(\mu_{\mathbf{k}}^{R}\right)^{2}\right]+\frac{1}{2}\left[\left(p_{\mathbf{k}}^{I}\right)^{2}+2 \frac{a^{\prime}}{a} \mu_{\mathbf{k}}^{I} p_{\mathbf{k}}^{I}+k^{2}\left(\mu_{\mathbf{k}}^{I}\right)^{2}\right]\right\}
$$

que, como a Lagrangeana, consiste na soma de duas Hamiltonianas para a parte real e para a parte imaginária.

Nosso intuito é comparar essa Hamiltoniana que descreve um campo escalar em um espaço-tempo curvo com a Hamiltoniana do efeito Schwinger (5.12). Essas Hamiltonianas diferem por termos lineares no momento canonicamente conjugado. Contudo, essa diferença é apenas aparente e essas Hamiltonianas descrevem processos semelhantes. Podemos ver que se incluirmos na Lagrangeana em (7.4) um termo de derivada total da forma:

$$
\frac{1}{2} \frac{d}{d \eta}\left[\frac{a^{\prime}}{a}\left(\mu_{\mathrm{k}}^{R}\right)^{2}+\frac{a^{\prime}}{a}\left(\mu_{\mathbf{k}}^{I}\right)^{2}\right],
$$

a Lagrangeana (e a ação) toma a forma quadrática:

$$
S=\frac{1}{2} \int d \eta \int_{\mathbb{R}_{+}^{3}} d^{3} k\left\{\left(\mu_{\mathrm{k}}^{R^{\prime}}\right)^{2}+\left(\mu_{\mathrm{k}}^{I^{\prime}}\right)^{2}-\left(\frac{a^{\prime \prime}}{a}-k^{2}\right)\left[\left(\mu_{\mathrm{k}}^{R}\right)^{2}+\left(\mu_{\mathrm{k}}^{I}\right)^{2}\right]\right\}
$$

em que $\omega_{k}^{2}(\eta)=k^{2}-a^{\prime \prime} / a$, com Hamiltoniana correspondente:

$$
H=\int_{\mathbb{R}_{+}^{3}} d^{3} k\left\{\frac{1}{2}\left[\left(p_{\mathbf{k}}^{R}\right)^{2}+\left(\frac{a^{\prime \prime}}{a}-k^{2}\right)\left(\mu_{\mathbf{k}}^{R}\right)^{2}\right]+\frac{1}{2}\left[\left(p_{\mathbf{k}}^{I}\right)^{2}+\left(\frac{a^{\prime \prime}}{a}-k^{2}\right)\left(\mu_{\mathbf{k}}^{I}\right)^{2}\right]\right\} .
$$

Agora podemos ver que a Hamiltoniana e a Lagrangeana acima têm a mesma forma que a do efeito Schwinger estático, (5.8) e (5.12), de um oscilador paramétrico com freqüência dependente do tempo, que equivale ao processo de um campo escalar quântico em presença de um campo externo. Isso pode ser visto também anteriormente quando calculamos as perturbações escalares e tensoriais durante a inflação no Capítulo 4 que obedecem ao mesmo tipo de Lagrangeana que a do efeito Schwinger.

Contudo, como esse campo externo é diferente em cada caso, a dependência temporal da freqüência também é diferente em ambos os casos, mas eles descrevem essencialmente o mesmo mecanismo. Assim, as Hamiltonianas (7.6) e (7.9) são fisicamente idênticas e descrevem a mesma teoria em duas versões conectadas por uma transformação canônica: (7.6) tem a forma que emerge naturalmente no caso das perturbações cosmológicas e (7.9) 
tem a mesma forma que o efeito Schwinger estático. Isso nos faz ver que a visão de um campo em presença de um fundo dependente do tempo e a de um campo interagindo com um potencial gravitacional são análogas. Esse é um dos resultados centrais dessa dissertação. A análise da criação de partículas a partir daqui é padrão e encontra-se na literatura [88], contudo a faremos de forma mais detalhada e diferenciando alguns detalhes. Ela segue também as linhas da análise utilizada para o efeito Schwinger estático.

\subsubsection{Perturbações inflacionárias a la Schwinger}

Adotando agora o modelo inflacionário, em que o campo escalar $\Phi$ é na verdade o campo do inflaton, $\varphi$, e temos também um termo potencial, que no caso vamos adotar o potencial quadrático da inflação caótica, $V(\varphi)=\frac{1}{2} m^{2} \varphi^{2}$. Então a Hamiltoniana correspondente à ação de Einstein-Hilbert de um campo escalar real em um espaço-tempo curvo pode ser escrita como:

$$
S=\frac{1}{2} \int d \eta \int_{\mathbb{R}_{+}^{3}} d^{3} k\left\{\left(\varphi_{\mathbf{k}}^{R^{\prime}}\right)^{2}+\left(\varphi_{\mathbf{k}}^{I^{\prime}}\right)^{2}-\left(k^{2}+a^{2} m^{2}-\frac{a^{\prime \prime}}{a}\right)\left[\left(\varphi_{\mathbf{k}}^{R}\right)^{2}+\left(\varphi_{\mathbf{k}}^{I}\right)^{2}\right]\right\}
$$

que é equivalente à ação do efeito Schwinger estático, com freqüência $\omega_{k}^{2}(\eta)=k^{2}+$ $a^{2} m^{2}-a^{\prime \prime} / a$. A dependência temporal da freqüência é diferente, uma vez que, no período inflacionário o fator de escala cresce exponencialmente e dessa forma, em tempo conforme:

$$
a(\eta)=-\frac{1}{H_{I} \eta}
$$

Com isso, a freqüência é da forma:

$$
\omega_{k}^{2}(\eta)=k^{2}-\frac{1}{\eta^{2}}\left(2-\frac{m^{2}}{H_{I}^{2}}\right) .
$$

Essa massa é muito pequena, $m \ll m_{p l}$ como vimos no Capítulo da inflação.

Podemos ver aqui, novamente, a equivalência entre a equação de movimento que descreve o efeito Schwinger estático e a equação da qual poderemos calcular a criação de partículas da inflação (perturbações) acima. Essa equivalência pode ser vista também ao vermos que as perturbações cosmológicas, (4.111), que obedecem às mesmas equações de movimento de um oscilador paramétrico que o efeito Schwinger estático, confirmando que o mesmo mecanismo de criação de partículas está presente em todos os casos, somente mudando a origem da fonte clássica.

Temos que, no caso de efeito Schwinger estático, a freqüência dependia do tempo como $t$ e $t^{2}$ e no caso da inflação, temos que a dependência temporal vai como $1 / \eta^{2}$. Essa diferença na dependência temporal terá influência na taxa de criação de partículas que será diferente para os dois casos. Outra diferença que podemos notar é que no caso do 
efeito Schwinger, o campo tem um direção, no caso no eixo z. Dessa forma, a criação de partículas ocorre com diferentes intensidades em cada direção de $k$, sendo preferencial na direção z. Contudo, no caso da inflação, como a expansão é homogênea e isotrópica, ela ocorre em todas as direção. Assim, podemos dizer em nossa analogia que o campo gravitacional age em todas as direções e assim a criação de partículas ocorre em todas as direções, ou seja para todo $k$.

Derivando de (7.4) as equações de movimento para a inflação, temos que:

$$
\varphi_{\mathbf{k}}^{\prime \prime}+\omega_{k}^{2}(\eta) \varphi_{\mathbf{k}}^{2}=0
$$

que é a equação de um oscilador harmônico paramétrico como no caso do efeito Schwinger.

\section{Quantização Canônica}

Podemos fazer o procedimento de quantização canônica do caso inflacionário de maneira análoga ao feito para o efeito Schwinger estático, uma vez que representam o mesmo mecanismo. Assim, vamos promover o campo quântico, que é nossa variável canônica, e o momento canonicamente conjugado a operadores e impor as regras de comutação em tempos iguais:

$$
\left[\hat{\varphi}(\eta, \mathbf{x}), \hat{p}^{\dagger}\left(\eta, \mathbf{x}^{\prime}\right)\right]=i \delta\left(\mathbf{x}-\mathbf{x}^{\prime}\right),
$$

em que todas as outras relações de comutação são nulas.

Podemos expandir esses operadores quânticos da forma:

$$
\hat{\varphi}(\eta, \mathbf{x})=\int \frac{d^{3} k}{(2 \pi)^{3 / 2}}\left[\varphi_{\mathbf{k}}(\eta) \hat{a}_{\mathbf{k}} \mathrm{e}^{-i \mathbf{k x}}+\varphi_{\mathbf{k}}^{*}(\eta) \hat{a}_{\mathbf{k}}^{\dagger} e^{i \mathbf{k x}}\right],
$$

em que $\hat{a}_{\mathbf{k}}$ e $\hat{a}_{\mathbf{k}}^{\dagger}$ são respectivamente os operadores de aniquilação e criação de partículas agindo no vácuo da representação de Fock. Estamos fazendo aqui a segunda quantização, em que as regras de comutação das variáveis de campo são traduzidas para esses operadores, de forma que eles obedecem as regras de comutação em tempo iguais:

$$
\left[\hat{a}_{\mathbf{k}}, \hat{a}_{\mathbf{k}^{\prime}}^{\dagger}\right]=\delta\left(\mathbf{k}-\mathbf{k}^{\prime}\right)
$$

em que todas as outras comutações são nulas. Lembrando que as variáveis obedecem à relação de normalização, vinda da relação de comutação:

$$
\varphi_{\mathbf{k}}^{\prime} \varphi_{\mathbf{k}}^{*}-\varphi_{\mathbf{k}}^{*^{\prime}} \varphi_{\mathbf{k}}=i
$$

que representa o wronskiano das soluções clássicas, que nos permite fixar a amplitude de 
$\varphi_{\mathbf{k}}(\eta)$ que é compatível com o princípio de incerteza de Heisenberg.

Com esses operadores, a Hamiltoniana do sistema pode ser escrita na forma diagonal:

$$
\hat{H}_{\mathbf{k}}=\omega_{\mathbf{k}}^{2}(\eta) \hat{a}_{\mathbf{k}}^{\dagger} \hat{a}_{\mathbf{k}}
$$

O próximo passo da quantização canônica é definir o espaço de Fock no qual as variáveis agem. Como vimos nos capítulos anteriores, para um campo escalar, podemos definir um único estado de vácuo, como sendo:

$$
\hat{a}_{\mathbf{k}}|0\rangle=0, \forall \mathbf{k}
$$

ou seja, o estado que não contém partículas (ou antipartículas). Os operadores de criação criam um estado que contém partículas e antipartículas, respectivamente,

$$
\hat{a}_{\mathbf{k}}^{\dagger}|0\rangle=|1 \mathbf{k}\rangle
$$

e todos os outros estados do sistema podem ser construídos a partir desse operador de criação.

Como visto anteriormente, no caso em presença de um campo externo, a invariância translacional, no espaço e no tempo, é quebrada e assim não podemos definir uma base ortonormal única. Ou seja, o estado de vácuo definido em (7.19) é agora dependente da base em que a quantização foi feita e tem direção temporal definida e é isso que possibilita a criação de partículas.

Nosso interesse é calcular o número de partículas criadas. Como feito no Capítulo 4, na Seção 4.2 e no Capítulo 5, sabemos que o valor do operador número de partículas em um tempo inicial $\eta_{1}$, pode ser igual a zero para o estado de vácuo $\left|0, \eta_{1}\right\rangle$ mas diferente de zero para um instante posterior $\eta_{2}$ :

$$
\left\langle 0, \eta_{2}\left|\hat{N}_{\mathbf{k}}\left(\eta_{1}\right)\right| 0, \eta_{2}\right\rangle \neq 0
$$

Podemos relacionar os operadores de criação e aniquilação em tempos diferentes por um transformada de Bogoliubov do tipo (5.88):

$$
\left(\begin{array}{c}
\hat{a}_{\mathbf{k}}\left(\eta_{2}\right) \\
\hat{a}_{\mathbf{k}}^{\dagger}\left(\eta_{2}\right)
\end{array}\right)=\left(\begin{array}{cc}
\alpha_{k} & \beta_{k}^{*} \\
\beta_{k} & \alpha_{k}^{*}
\end{array}\right)\left(\begin{array}{c}
\hat{a}_{\mathbf{k}}\left(\eta_{1}\right) \\
\hat{a}_{\mathbf{k}}^{\dagger}\left(\eta_{1}\right)
\end{array}\right),
$$

em que os coeficientes de Bogoliubov $\alpha_{k}$ e $\beta_{k}$, das relações de comutação (7.16), obedecem a:

$$
\left|\alpha_{k}\right|^{2}-\left|\beta_{k}\right|^{2}=1
$$

Dessa forma, sabemos que o número de partículas criadas é dado por: 


$$
\left\langle N_{\mathbf{k}}^{(1)}\right\rangle_{\eta_{2}}=\left\{\begin{array}{l}
\left|\beta_{k}\left(\eta_{1}, \eta_{2}\right)\right|^{2} \\
\left\langle N_{\mathbf{k}}\left(t_{1}\right)\right\rangle+\left|\beta_{\mathrm{b}}\right|^{2}\left(1+2\left\langle N_{\mathbf{k}}\left(t_{1}\right)\right\rangle\right), \text { se }\left\langle N_{\mathbf{k}}\left(t_{1}\right)\right\rangle \neq 0
\end{array}\right.
$$

como no caso do efeito Schwinger estático (5.94).

Logo, para determinarmos o número de partículas criadas na inflação precisamos determinar os coeficientes de Bogoliubov. Para isso vamos utilizar o método WKB, como feito na Seção 5.2.2.

\section{Criação de partículas na inflação: aproximação WKB e as condições iniciais}

Vamos utilizar a aproximação WKB para determinarmos os coeficientes de Bogoliubov. Contudo, esse método no caso inflacionário nos fornece outras informações muito importantes: ele define os estados de vácuo adiabáticos inicial e final, que não são conhecidos em uma teoria do universo primordial como a inflação.

A solução da equação (7.13) pode ser escrita como uma combinação linear de funções de Hankel de primeira e segunda espécie da forma [50]:

$$
\varphi_{\mathbf{k}}(\eta)=\sqrt{-\eta}\left[C_{1} H_{\nu}^{(1)}(-k \eta)+C_{2} H_{\nu}^{(2)}(-k \eta)\right]
$$

em que os coeficientes da combinação podem ser fixados pela normalização (7.17) e com coeficiente:

$$
\nu \equiv \sqrt{\frac{9}{4}-\frac{m^{2}}{H^{2}}}
$$

Como mencionamos nos capítulos anteriores, os conceitos de vácuo e de partícula em espaços-tempo curvos não estão bem definidos, uma vez que detectores em movimento acelerado podem detectar partículas que detectores em repouso não detectam [62]. Para nossa análise ser consistente, então, temos que ter uma boa definição do vácuo adiabático do qual as partículas serão criadas. Como o conceito de partículas e vácuo é bem definido no TQC em um espaço-tempo de Minkowski, podemos então descrever a criação de partículas pela expansão acelerada entre dois instantes em que podemos aproximar o universo por um universo de Minkowski. Pensando, agora, como um problema de o campo do inflaton imerso no campo gravitacional da inflação, podemos pensar isso como se a criação tomasse lugar entre dois instantes em que esse campo gravitacional fosse inexistente, o que sabemos ser impossível, ou mais fraco, tal que o processo de criação de partículas não agisse. Se a expansão do universo é muito pequena, temos que o conceito número de partículas é um invariante adiabático, sem levar em conta a quantidade total de expansão. Isso é consistente com o que vimos no Capítulo 4, que as flutuações quânticas no começo da inflação se comportam como ondas planas num espaço-tempo de Minkowski. Esse 
resultado pode ser visto também da solução da equação de movimento (7.25), em que, no limite assintótico para $z \equiv-k \eta \gg 1$, nos fornece:

$$
\varphi_{\mathbf{k}}(\eta) \propto \exp \left[ \pm i\left(z-\frac{\pi \nu}{2}-\frac{\pi}{4}\right)\right]
$$

que é uma solução de onda plana.

Sabemos que a aproximação WKB é válida no limite em que:

$$
\left|Q / \omega^{2}\right| \ll 1
$$

com $Q$ construído da forma (5.128), e vem da equação :

$$
\varphi_{\mathbf{k}}^{\prime \prime}+\left(\omega_{\mathbf{k}}^{2}(\eta)-Q\right) \varphi_{\mathbf{k}}=0 .
$$

Para o nosso caso, em que a freqüência é dada por (7.12), temos que:

$$
\left|\frac{Q}{\omega^{2}}\right|=\frac{1}{4 \alpha^{2}}\left|\frac{1-6(k \eta / \alpha)^{2}}{\left[1-(k \eta / \alpha)^{2}\right]^{3}}\right|,
$$

em que $\alpha=\left(m^{2} / H_{I}^{2}\right)-2$.

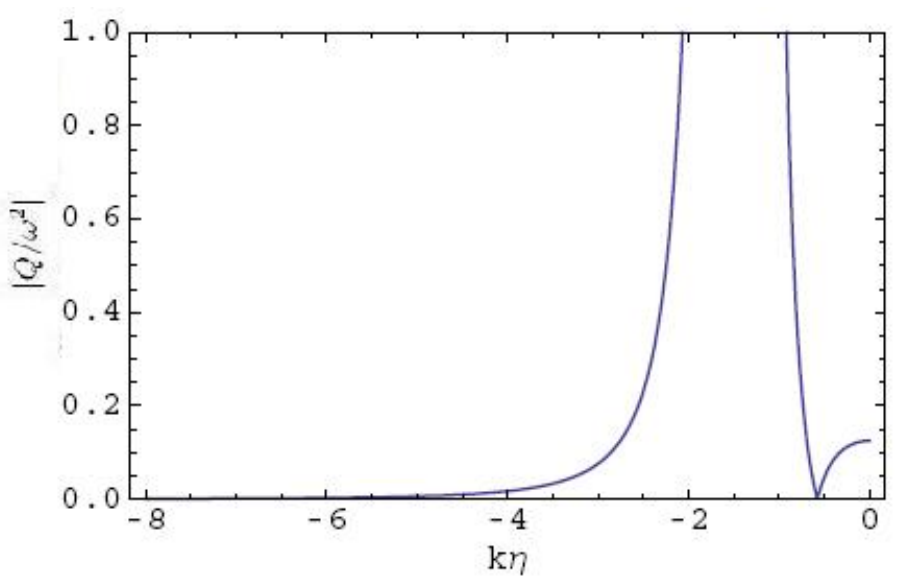

Figura 7.1: Gráfico da condição de adiabaticidade para $m^{2} \sim 10^{-25}$, ou seja, $\alpha \sim 2$. Podemos ver que a adiabaticidade é quebrada em um intervalo finito, onde ocorre a criação de partículas, e que para $k \eta \rightarrow-\infty$, a adiabaticidade não é violada.

Podemos ver na Figura 7.1, que a condição WKB é violada em instantes de tempo finitos, sendo que no infinito passado, $k \eta \rightarrow-\infty$, temos que a condição de adiabaticidade é bem definida e válida, representando um estado de vácuo do tipo Minkowski.

Dessa forma, solução WKB é dada por: 


$$
\begin{aligned}
\varphi_{\mathbf{k}}(\eta) & \simeq \alpha_{k} \varphi_{w k b, \mathbf{k}}(\eta)+\beta_{k} \varphi_{w k b, \mathbf{k}}^{*}(\eta) \\
& =\frac{\alpha_{k}}{\sqrt{2 \omega}} \exp \left(-i \int_{\eta_{i n i}}^{\eta} \omega\left(\eta^{\prime}\right) d \eta^{\prime}\right)+\frac{\beta_{k}}{\sqrt{2 \omega}} \exp \left(+i \int_{\eta_{i n i}}^{\eta} \omega\left(\eta^{\prime}\right) d \eta^{\prime}\right)
\end{aligned}
$$

em que função de modo WKB, solução de (7.29), é dada por:

$$
\phi_{w k b, \mathbf{k}}=\frac{1}{\sqrt{2 \omega_{\mathbf{k}}}} \exp \left( \pm \int \omega_{\mathbf{k}} d \eta\right)
$$

Para o estado adiabático inicial, $k \eta \rightarrow-\infty$, que a solução geral (7.25) pode ser escrita utilizando as expansões assintóticas da função de Hankel nesse limite, dadas por (C.1) e (C.2). Podemos determinar os coeficientes $C_{1}$ e $C_{2}$ para um universo inflacionário com $\omega_{k}^{2}(\eta)=k^{2}-2 / \eta^{2}$, tomando $\alpha_{k}=1$ e $\beta_{k}=0$ e comparando com a solução geral assintótica. Assim, especificamos o estado de vácuo inicial:

$$
\phi_{w k b, \mathbf{k}}(\eta)=\frac{1}{\sqrt{2 k}}\left(1-\frac{i}{k \eta}\right) \exp (-i k \eta)
$$

que é o vácuo de Bunch-Davies [62]. No Capítulo 4 já havíamos visto que o estado inicial da inflação era o vácuo de Bunch-Davies. Esse é um resultado de extrema importância que decorre naturalmente da análise WKB, uma vez que a fase inflacionária implica na validação do método WKB para o universo jovem e, conseqüentemente, garante que podemos escolher um estado de vácuo inicial bem definido, no caso o vácuo de Bunch-Davies.

Apesar de podermos determinar um estado de vácuo adiabático inicial, pelo método WKB, podemos ver que para $k \eta \rightarrow 0$, temos que $\left|Q / \omega^{2}\right| \rightarrow 1 / 4 \alpha^{2}$. Isso pode ser visto na Figura 7.1.2, em que $\left|Q / \omega^{2}\right| \rightarrow 1 / 8$. Nesse caso, a aproximação WKB não é válida, uma vez que a condição adiabática não é obedecida. Isso significa que no estado final a curvatura ainda é sentida e não podemos definir um estado de vácuo único nesse limite.

Sabendo que o comprimento de onda correspondente a um modo de Fourier é dado por:

$$
\lambda(\eta)=\frac{2 \pi}{k} a(\eta)
$$

em que $k$ é o número de onda comóvel, e que o raio de Hubble é escrito como:

$$
l_{H}(\eta)=\frac{a^{2}}{a^{\prime}},
$$

os comportamentos nos limites $k \eta \rightarrow-\infty$ e $k \eta \rightarrow 0$, correspondem às escalas $\lambda \ll l_{H}$ e $\lambda \gg l_{H}$, respectivamente. Dessa forma, podemos ver que a aproximação WKB é válida dentro do horizonte, uma vez que como o comprimento de onda é muito pequeno em 
relação ao raio de Hubble $l_{H}$, que caracteriza a curvatura do espaço-tempo, o modo de Fourier se comporta como se estivesse em um espaço-tempo plano (Minkowski), não sentindo, assim, a curvatura do espaço-tempo. Contudo, para o limite em que $k \eta \rightarrow-0$, que corresponde ao caso em que $\lambda \gg l_{H}$, os modos de Fourier encontram-se fora do raio de Hubble e a curvatura do espaço-tempo é sentida e a aproximação WKB não é mais válida ${ }^{1}$. Logo, não é possível aplicarmos a aproximação WKB, como feito no caso do efeito Schwinger, para determinarmos os estados iniciais e finais adiabáticos entre os quais a criação de partículas ocorre ${ }^{2}$.

Como não podemos determinar um estado adiabático final para o período inflacionário, não podemos calcular a criação de partículas nesse sistema. Ainda assim é possível calcular o espectro de potências no fim da inflação.

Contudo, existe uma maneira de utilizarmos o aproximação WKB para computarmos a criação de partículas durante a inflação. Se seguirmos a evolução do universo após a inflação, até que a unicidade da escolha do vácuo seja restaurada, poderemos definir um estado adiabático no futuro. Dessa forma, definimos o estado final adiabático como o instante em que a inflação termina e a expansão deixa de ser exponencial e passa a ser mais fraca. Essa expansão poderia ser a de um universo dominado por matéria ou radiação, correspondente a quando os modos reentram o raio de Hubble. Como vimos na Seção 3.3 do Capítulo 3, para o potencial $V(\varphi)=m^{2} \varphi^{2} / 2$, o fator de escala no início do reaquecimento, antes do universo ser termalizado, varia como $a(t)=a_{0}^{m} t^{2 / 3}$, imitando um universo composto por matéria não relativística. Partículas também são criadas durante o reaquecimento, mas podemos definir como sendo o estágio adiabático final da inflação como sendo o momento em que a inflação acaba e o reaquecimento começa, quando o inflaton chega no mínimo global de seu potencial.

Teremos, então um período inflacionário que evolui como $a(\eta) \propto 1 / \eta,-\infty<\eta<0$, seguido de um período dominado pela matéria que evolui como $a(\eta) \propto \eta^{2},-0 \leq \eta<\infty$. Podemos determinar, assim, o estado inicial adiabático, vindo da aproximação adiabática válida para o estágio inflacionário em $\eta \rightarrow-\infty$ e o estado adiabático final (que não é vácuo, mas um estado adiabático em que partículas não são criadas), em que a aproximação adiabática para um universo como o dominado por matéria:

$$
\left|\frac{Q}{\omega^{2}}\right|=\frac{1}{4}\left|\frac{1-6(k \eta)^{2}}{\left[1-(k \eta)^{2}\right]^{3}}\right| \rightarrow 0, k \eta \rightarrow \infty,
$$

\footnotetext{
${ }^{1}$ Esse problema é análogo ao conhecido problema de como a fórmula de Balmer para os níveis de energia do átomo de hidrogênio pode ser reconstruída pela aproximação WKB. Como no caso inflacionário, no limite em que $r \rightarrow 0$, a aproximação WKB não é mais válida [64].

${ }^{2}$ Apesar de apresentar esse problema, métodos baseados na aproximação WKB, são utilizados para o cálculo das perturbações cosmológicas, em que o problema em $k \eta \rightarrow 0$ é contornado. Eles representam um novo método de aproximação diferente da aproximação de rolagem lenta usualmente utilizada em cosmologia inflacionária. Podemos ver esse método em [65].
} 
é válida.

Dessa forma, os estágios adiabáticos inicial (in) e final (out) entre os quais ocorre a criação de partículas são bem definidos e a solução WKB é válida. Com isso, é possível calcular os coeficientes de Bogoliubov da maneira usual feita para o efeito Schwinger estático, e determinar o número de partículas criadas na inflação $N_{\text {infl }}=\left|\beta_{k}\right|^{2}$ (nenhuma partícula é criada ou está presente antes da inflação).

Se considerássemos um período inflacionário com potencial $V(\varphi)=\lambda \varphi^{4} / 4$, teríamos logo após a inflação um período de domínio da radiação [66]. Nesse caso, deveríamos considerar um estágio inflacionário seguido de um estágio dominado por radiação para o cálculo do número de partículas criadas.

Esse procedimento é feito também em alguns artigos considerando que o reaquecimento é instantâneo e, assim, temos a inflação seguida de um estágio dominado pela radiação [67, 68, 86]. Como calcularemos nessa dissertação explicitamente o número de partículas criadas durante o reaquecimento, esse quadro não interessa para nós.

\subsection{Criação de Partículas no Pré-aquecimento e o Efeito Schwinger Oscilatório}

Como vimos no Capítulo 3, pela teoria da inflação, praticamente todas as partículas elementares do universo foram criadas durante o reaquecimento. Esse período pode ser descrito em duas partes, em que a criação de partículas ocorre por um mecanismo perturbativo, que representa a fase final do reaquecimento, e um período não-perturbativo, que ocorre no inicio do reaquecimento. Esse período inicial não-perturbativo é chamado de pré-aquecimento e é o período mais bem entendido pelos cosmólogos até agora.

A criação de partículas, no pré-aquecimento, ocorre devido à oscilação do campo do inflaton em torno do mínimo de seu potencial. Esse mecanismo é modelado como o campo do inflaton interagindo com um campo escalar, uma vez que nesse período partículas escalares e de inflaton são criadas.

Vamos mostrar nessa seção que esse mecanismo de criação de partículas no préaquecimento pela oscilação do inflaton pode ser modelado de maneira análoga ao efeito Schwinger oscilatório (ESO), em que o campo quântico, que no caso do ESO é o campo escalar complexo, é o campo escalar e o campo externo oscilatório aplicado corresponde ao campo oscilatório do inflaton, que no ESO é um campo elétrico oscilatório.

\subsubsection{Pré-aquecimento}

O pré-aquecimento é o período em que partículas escalares são criadas. Dessa forma, para podemos descrever esse modelo, temos a Lagrangeana em que o inflaton é acoplado ao 
campo escalar $\left(\chi, m_{\chi}\right)[46]$ :

$$
\mathcal{L}=\frac{1}{2} \partial_{\mu} \varphi \partial^{\mu} \varphi-\frac{1}{2} m^{2} \varphi^{2}+\frac{1}{2} \partial_{\mu} \chi \partial^{\mu} \chi-\frac{1}{2} m_{\chi}^{2} \chi^{2}-\frac{1}{2} g^{2} \varphi^{2} \chi^{2}+\frac{1}{2} \xi R \chi^{2}
$$

em que $g$ e $\xi$ são constantes de acoplamento, $R$ é a curvatura do espaço-tempo.

Como feito no caso do reaquecimento perturbativo, consideramos o potencial quadrático da inflação caótica com quebra espontânea de simetria, $V(\varphi) \sim \frac{1}{2} m^{2}(\varphi-\sigma)^{2}$, em que, fazendo a translação $\varphi-\sigma \rightarrow \varphi$, o termo de interação $-\frac{1}{2} g^{2} \varphi^{2} \chi^{2}$ se transforma em $-\frac{1}{2} g^{2} \varphi^{2} \chi^{2}-\frac{1}{2} \sigma \varphi \chi^{2}-\frac{1}{2} g^{2} \sigma^{2} \chi^{2}$. No modelo de inflação caótica que utilizamos e em muitos outros, a amplitude inicial de oscilação do inflaton pode ser tão grande como $m_{p l}$, como visto no Capítulo 3. Dessa forma, essa amplitude inicial é muito maior que qualquer outro parâmetro como $\sigma$ e vamos considerar, então o modelo caótico simples da inflação sem quebra espontânea da simetria, $\operatorname{com} V(\varphi)=\frac{1}{2} m^{2} \varphi^{2}$.

Fazendo uma transformação de Fourier do campo:

$$
\chi(\mathbf{x}, t)=\int \frac{d^{3} k}{(2 \pi)^{3 / 2}} \chi_{\mathbf{k}}(t) \mathrm{e}^{-i \mathbf{k x}},
$$

podemos escrever as equações de movimento da forma:

$$
\ddot{\chi}_{\mathbf{k}}+3 \frac{\dot{a}}{a} \chi_{\mathbf{k}}+\left(k^{2}+m_{\chi}^{2}-\xi R+g^{2} \varphi^{2}\right) \chi_{\mathbf{k}}=0 .
$$

Vamos considerar também que somente criamos partículas leves durante o pré-aquecimento, ou seja, que quando $\varphi=0$, a massa do campo $\chi$ é igual a zero. Ou seja, que $m \gg m_{\chi}$. Como podemos ver em [46], a produção de partículas muito massivas, com $m \ll m_{\chi}$ é possível, mas fortemente suprimida. Por isso, durante o pré-aquecimento para que seja possível termos a produção de partículas necessária para termos o universo térmico atual, as partículas criadas têm que ser partículas leves para que o processo seja eficiente.

A criação de partículas é mais eficiente se $k / a \gg m$, sendo que, após a inflação $m \gg H$, como vimos na Seção 3.3. Assim, podemos considerar uma primeira aproximação em que ignoramos a expansão do universo fazendo $a=1$. Dessa forma, obtemos a Lagrangeana para o sistema da forma:

$$
\mathcal{L}=\frac{1}{2} \partial_{\mu} \varphi \partial^{\mu} \varphi-\frac{1}{2} m^{2} \varphi^{2}+\frac{1}{2} \partial_{\mu} \chi \partial^{\mu} \chi-\frac{1}{2} g^{2} \varphi^{2} \chi^{2} .
$$

Para podermos estudar a criação de partículas, vamos fazer a quantização canônica do sistema. 


\section{Quantização Canônica}

Como feito inúmeras vezes nessa dissertação, para quantizarmos canonicamente esse sistema, definimos o momento canonicamente conjugado a $\chi$ como:

$$
p=\frac{\partial \mathcal{L}}{\partial \partial_{0} \chi}=\partial_{0} \chi=\dot{\chi}
$$

e promovemos as variáveis $\chi$ e $p$ a operadores, $\hat{\chi}$ e $\hat{p}$ (analogamente, no espaço de Fourier, $\chi_{\mathbf{k}} \rightarrow \hat{\chi}_{\mathbf{k}}$ e $p_{\mathbf{k}}=\dot{\chi}_{\mathbf{k}} \rightarrow \hat{p}_{\mathbf{k}}$ ). Esses operadores obedecem às relações de comutação:

$$
[\hat{\chi}(t, \mathbf{x}), \hat{\Pi}(t, \mathbf{y})]=i \delta(\mathbf{x}-\mathbf{y})
$$

com todos os outros comutadores iguais a zero ou, para as variáveis no espaço de Fourier, $\left[\hat{\chi}_{\mathbf{k}}, \hat{p}_{\mathbf{k}^{\prime}}\right]=\delta\left(\mathbf{k}-\mathbf{k}^{\prime}\right)$, com todos os outros comutadores iguais a zero.

Escrevendo o campo escalar quântico $\chi$, na representação de Heisenberg:

$$
\chi(\eta, \mathbf{x})=\frac{1}{(2 \pi)^{3 / 2}} \int d^{3} k\left[\hat{a}_{\mathbf{k}} \chi_{\mathbf{k}}(t) \mathrm{e}^{-i \mathbf{k x}}+\hat{a}_{\mathbf{k}}^{\dagger} \chi_{\mathbf{k}}^{*}(t) \mathrm{e}^{i \mathbf{k x}}\right]
$$

em que passamos para os operadores de criação e aniquilação o papel de operador. Aplicando as regras de quantização, teremos que esses operadores têm que obedecer a:

$$
\left[\hat{a}_{\mathbf{k}}, \hat{a}_{\mathbf{k}^{\prime}}^{\dagger}\right]=\delta\left(\mathbf{k}-\mathbf{k}^{\prime}\right)
$$

com os outros comutadores iguais a zero. Essas regras de comutação somente são válidas se os modos $\chi_{\mathbf{k}}(t)$ obedecerem à relação de normalização, vinda da relação de comutação:

$$
\dot{\chi}_{\mathbf{k}} \chi_{\mathbf{k}}^{*}-\dot{\chi}_{\mathbf{k}}^{*} \chi_{\mathbf{k}}=2 i
$$

que representa o wronskiano das soluções clássicas. Essas variáveis obedecem, então, a equação de movimento:

$$
\ddot{\chi}_{\mathbf{k}}+\left(k^{2}+m_{\chi}^{2}+g^{2} \varphi^{2}\right) \chi_{\mathbf{k}}=0
$$

em que $m_{\chi} \ll m$.

Como vimos na Seção 3.3 no Capítulo 3, o campo escalar é oscilatório, da forma:

$$
\varphi(t)=\Phi(t) \operatorname{sen}(m t)
$$

e, dessa forma, a equação de movimento é dada por:

$$
\ddot{\chi}_{\mathbf{k}}+\left[k^{2}+m_{\chi}^{2}+g^{2} \Phi^{2} \operatorname{sen}^{2}(m t)\right] \chi_{\mathbf{k}}=0 .
$$

Essa equação descreve um oscilador paramétrico com freqüência dependente do tempo, 
$\omega_{\mathbf{k}}^{2}=k^{2}+m_{\chi}^{2}+g^{2} \Phi^{2} \operatorname{sen}^{2}(m t)$. Ela pode ser escrita como uma equação de Mathieu, da forma:

$$
\frac{d^{2} \chi_{\mathbf{k}}}{d z^{2}}+\left(A_{\mathbf{k}}-2 q \cos 2 z\right) \chi_{\mathbf{k}}=0
$$

em que $z=m t$ e:

$$
A_{k}=\frac{k^{2}+m_{\chi}^{2}}{m^{2}}+2 q, q=\frac{g^{2} \Phi^{2}}{4 m^{2}} .
$$

Podemos ver aqui que a descrição do pré-aquecimento é equivalente à descrição do efeito Schwinger oscilatório. Como vimos no Capítulo 6, no efeito Schwinger oscilatório a dinâmica do sistema também é escrita por uma equação de Mathieu, quando suprimimos a criação de partículas na direção $z$. Dessa forma, podemos dizer que ambos os sistemas são equivalentes.

Essa afirmação pode ser feita também se examinarmos as Lagrangeana que descrevem ambos os sistemas. Para o efeito Schwinger oscilatório a Lagrangeana que descreve o sistema é dada por:

$$
\mathcal{L}=\frac{1}{2} \partial_{\mu} \phi \partial^{\mu} \phi^{*}-\frac{1}{2} m^{2} \phi \phi^{*}+\frac{i e}{2}\left[\left(\partial_{\mu} \phi\right) A^{\mu} \phi^{*}-A_{\mu} \phi\left(\partial^{\mu} \phi^{*}\right)\right]+\frac{1}{2} e^{2} A^{2} \phi \phi^{*}
$$

em que $\phi$ é um campo escalar complexo e o campo externo é dado por $A_{\mu}$. Vemos que essa Lagrangeana diferencia-se da Lagrangeana (7.41) por um termo cinético e do potencial do campo eletromagnético externo. Isso ocorre, pois no caso do efeito Schwinger oscilatório, utilizamos o calibre de Lorentz, em que $\partial_{\mu} A^{\mu}=0$ e o termo do potencial é nulo, uma vez que para o campo eletromagnético a massa é nula. Contudo, ainda há mais um termo que diferencia as duas descrições dos sistemas que está no termo que contém um derivada primeira. Podemos mostrar facilmente que, se considerarmos um campo escalar não complexo no efeito Schwinger oscilatório, análogo ao do pré-aquecimento, da forma:

$$
\mathcal{L}=\frac{1}{2} \partial_{\mu} \phi \partial^{\mu} \phi-\frac{1}{2} m^{2} \phi^{2}+i e\left(\partial_{\mu} \phi\right) A^{\mu} \phi+\frac{1}{2} e^{2} A^{2} \phi^{2} .
$$

Esse termo de derivada primeira do campo pode ser retirado da Lagrangeana subtraindo uma derivada total da forma:

$$
\frac{1}{2} \partial_{\mu}\left(i e A^{\mu} \phi^{2}\right)
$$

Esse termo equivale a soma de um termo nulo uma vez que, pelo teorema da (quadri)divergência, o termo dentro da derivada total se anula na superfície infinita. 
Assim, as Lagrangeanas que descrevem ambos os sistemas é a de um campo quântico interagindo com um potencial externo de forma quadrática. Dessa forma, dizemos que esses sistemas são equivalentes e que podemos entender a criação de partículas no préaquecimento como um efeito Schwinger oscilatório com potencial gravitacional.

Isso nos faz ver que se esses sistemas são equivalentes, a detecção experimental do efeito Schwinger oscilatório, ou de seu análogo o efeito Casimir dinâmico, seria uma sustentação desse mecanismo de criação de partículas no universo primordial.

\subsubsection{Criação de partículas}

Uma vez que ambos os sistemas do pré-aquecimento e do efeito Schwinger oscilatório têm a mesma descrição, vamos estudar a criação de partículas no pré-aquecimento da mesma forma feita no efeito Schwinger oscilatório. Logo, partindo da equação de Mathieu para o pré-aquecimento (7.50) podemos analisar o comportamento como feito na Seção 6.2. Como vimos, existe uma quantidade muito grande de soluções para esta equação, ambas periódicas e não-periódicas que não podem ser escritas em termo de funções elementares e como os coeficientes da equação são periódicos, a solução pode ser determinada pelo teorema de Floquet. Como no efeito Schwinger oscilatório, essas soluções apresentam uma estrutura de bandas de ressonância que, dependendo dos valores de $A$ e $q$, correspondem às bandas de instabilidade e estabilidade, como representado na figura 6.2.

Nas bandas de instabilidade, temos uma ressonância estreita (narrow), em que $g \Phi<m$ ou $q \ll 1$, ou seja, a amplitude da oscilação é menor que a freqüência. Nesse regime os modos são amplificados exponencialmente. Para $g \Phi>m$ ou $q \gg 1$, temos um regime de ressonância ampla ou larga (broad) em que a ressonância ocorre para uma faixa ampla de valores de $k$ e a amplitude de oscilação é maior que sua freqüência. Assim, como no efeito Schwinger oscilatório, a criação de partículas no pré-aquecimento é mais eficiente no período de ressonância ampla (broad).

O regime de ressonância estreita (narrow) ocorre quando a freqüência de oscilação é maior que sua amplitude, ou seja, o inflaton oscila mais rapidamente em torno do mínimo de seu potencial e a amplitude dessas oscilações é menor. Dessa forma, podemos ver que esse regime ocorre depois de um certo número de oscilações do inflaton, em que sua energia já decaiu quase completamente com a criação de partículas. Podemos analisar esse período pelos métodos usuais de tratamento da equação de Mathieu em que aplica-se o teorema de Floquet, que afirma que uma equação diferencial com coeficientes periódicos (a de Mathieu) admite uma solução periódica da forma:

$$
\chi_{\mathbf{k}}=u(z, \sigma) e^{\mu_{k} z},
$$

em que $\mu_{N}$ é o índice de Floquet e $\sigma$ é o valor de $k^{2}+m^{2}$ para o qual a solução é computada. Do gráfico na Figura 6.2, temos as regiões de instabilidade e estabilidade da equação de 
Mathieu, que correspondem a estrutura de bandas da ressonância. Assim, para $q \ll 1$, a ressonância é mais considerável para os modos com $k^{2} \sim m^{2}(1-2 q \pm q)$, que corresponde à primeira banda de ressonância, ou à primeira banda de instabilidade. Vamos considerar o centro da banda de ressonância, com $k \sim m$. Nesse caso, a solução é dada por:

$$
\chi_{\mathbf{k}} \sim \mathrm{e}^{\mu_{k} m t} \sim \mathrm{e}^{\frac{q z}{2}} \sim \mathrm{e}^{\frac{g^{2} \Phi^{2}}{8 m}}
$$

ou seja, esse modo cresce exponencialmente. Nesse caso, o número de partículas criadas, que corresponde a um processo de ressonância com decaimento de duas partículas de inflaton $\varphi$ em duas partículas escalares $\chi$ com momento $k \sim m$, cresce exponencialmente [46]:

$$
n_{\mathbf{k}}=\frac{\omega_{\mathbf{k}}}{2}\left(\frac{\left|\dot{\chi}_{\mathbf{k}}\right|^{2}}{\omega_{\mathbf{k}}^{2}}+\left|\chi_{\mathbf{k}}\right|^{2}\right)-\frac{1}{2} \sim \mathrm{e}^{2 \mu_{k} m t} \sim \mathrm{e}^{\frac{g^{2} \Phi^{2}}{4 m}} .
$$

Podemos ver na Figura 7.2, essa criação de partículas, retirada de [46].

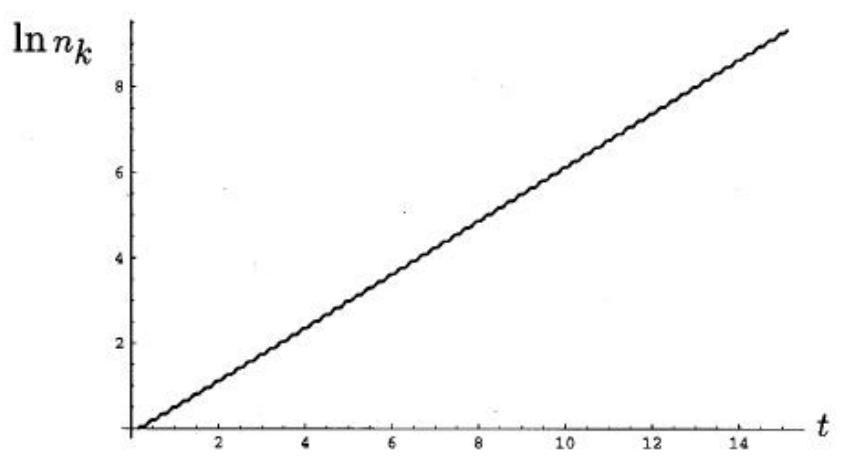

Figura 7.2: Figura retirada de [46], que mostra o logaritmo do número de partículas $\chi$ criadas em um modelo sem expansão do universo. Esse é um simulação em que $q \sim 0,1$ e o tempo é dado em termos do número de oscilações do inflaton, $m / 2 \pi$. As pequenas oscilações no gráfico linear, que representa um crescimento exponencial do número de partículas, se deve a cada oscilação do campo do inflaton.

A criação de partículas nesse regime não é eficiente, uma vez que $\mu_{\mathbf{k}} \ll 1$, sendo que o número de partículas criadas por esse regime não seria o suficiente, contando a duração do reaquecimento, para reaquecer o universo.

Dessa forma, temos que considerar o regime de ressonância larga (broad), em que a criação de partículas é eficiente e que é responsável pela criação de praticamente todas as partículas elementares do universo. 


\section{Criação de partículas no regime de ressonância larga}

O regime de ressonância larga, corresponde ao caso em que a amplitude da oscilação é grande. Esse ocorre nos estágios iniciais do reaquecimento, em que o inflaton começou a oscilar em torno de seu potencial e essa oscilação tem amplitude grande e freqüência pequena. Assim, nesse regime o índice de Floquet da solução é grande para uma grande gama de valores de $k$, fazendo com que a criação de partículas seja grande e eficiente.

Como $q \gg 1$, temos que as bandas de ressonância ocorrem acima da linha $A=$ $2 q$ no gráfico de instabilidade/estabilidade da equação de Mathieu, que corresponde a modos com $k^{2} / m^{2}=A-2 q$. Podemos ver esse regime no gráfico (quadro da direita) de instabilidade/estabilidade da Figura 6.2, em que a linha $A=2 q$ é traçada, correspondente a $k=0$. As curvas nas regiões brancas correspondem aos valores de $\mu_{\mathbf{k}}$ dentro dessas bandas de instabilidade.

A criação de partículas no regime de ressonância ampla ocorre durante uma parte da oscilação do inflaton, quando $1-\cos z \sim q^{-1}$ [45]. Uma massa efetiva é induzida pelo acoplamento dos campos e essa é sempre menor que a massa do inflaton, $m_{e f f, \chi}^{2}=$ $g^{2} \Phi^{2} / 2<m$. Dessa forma, o número de partículas criadas cresce exponencialmente com poucas oscilações do inflaton, o que leva a um crescimento explosivo do número de partículas, mesmo que a massa do campo escalar, $m_{\chi}$, seja maior que $m$, desde que a amplitude das oscilações sejam suficientemente grandes, $g \Phi \gg m_{\chi}^{2} / m$.

Contudo, nesse regime de ressonância não podemos aplicar os métodos usuais de resolução aplicado no regime estrito. Para isso, vamos adotar um método analítico para a resolução desse sistema [46]. Utilizamos o mesmo método analítico na resolução do efeito Schwinger oscilatório.

Como visto, utilizaremos o método analítico em que o campo $\chi_{\mathbf{k}}$ tem evolução adiabática dada pelos instantes em que o campo externo aplicado é igual a zero, $A\left(t_{j}\right)=$ $0, j=1,2,3 \ldots$, ou seja, o inflaton passa pelo mínimo do potencial, que é zero no nosso modelo de inflação caótica. A criação de partículas ocorre entre esses instantes, que têm evolução não-adiabática. Com isso, podemos formular a teoria da criação de partículas em um campo oscilatório em termos da aproximação adiabática (WKB) ou semi-clássica, em que o número de partículas criadas é dada pelo coeficiente de Bogoliubov derivado da passagem entre dois estados adiabáticos.

Como o inflaton oscila em torno do mínimo de seu potencial quadrático, quando ele passar no mínimo, o que equivale a não termos um campo externo aplicado no modelo do efeito Schwinger oscilatório, temos de $\varphi(t)=\Phi$ sen $(m t)$ que corresponde aos instantes $m t_{j}=\pi j$, para $j=1,2,3, \ldots$. Nesses instantes, a freqüência atinge seu mínimo dado por $\omega_{k}=\sqrt{k^{2}+m_{\chi}^{2}}$ e a amplitude de $\chi_{\mathbf{k}}$ é máxima.

Conforme o campo do inflaton oscila, o campo escalar de $\chi$ oscila diversas vezes, da ordem de $\mathcal{O}\left(q^{1 / 2}\right)$ vezes, de maneira que sua freqüência de oscilação, dada por $\omega_{\mathbf{k}}(t)$, 
é maior que a do inflaton no regime de ressonância ampla, ver Figura 7.3. A massa efetiva, $m_{e f f, \chi}(t)=g \varphi(t)$ é muito maior que a massa do inflaton $m$ durante grande parte do período de oscilação de $\varphi$. Dessa forma, podemos ter uma variação adiabática dessa massa efetiva durante grande parte desse período de oscilação.

Podemos ver isso de maneira mais precisa com o auxílio da aproximação WKB. A criação de partículas nessa aproximação ocorre entre os instante em que $\left|Q / \omega^{2}\right| \gtrsim 1$. No caso do pré-aquecimento, podemos estudar o comportamento adiabático utilizando a condição WKB de ordem mais baixa. Ou seja, a criação de partículas ocorre entre esses instantes de variação adiabática, em que:

$$
\left|\frac{\dot{\omega}}{\omega^{2}}\right| \gtrsim 1
$$

A variação adiabática é importante ao considerarmos esse limite semiclássico, uma vez que o número de partículas criadas é um invariante adiabático nos períodos em que a aproximação WKB é válida.

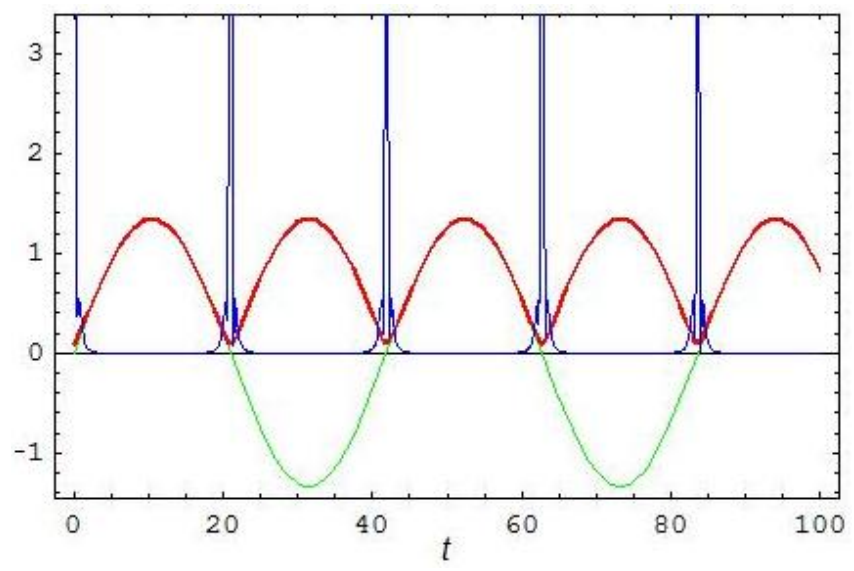

Figura 7.3: Gráfico que representa em verde a variação do potencial, em vermelho a freqüência e em azul o parâmetro WKB. A criação de partículas ocorre entre os instantes em que o parâmetro WKB é violado, que corresponde aos instantes em que a freqüência atinge seu mínimo.

Durante a maior parte do tempo temos que a freqüência varia lentamente, ou seja, que $|\dot{\omega}| \ll \omega^{2}$. Nesses instantes, o número de partículas é conservado e é um invariante adiabático. Somente podemos computar o número de partículas nesses instantes.

Para os instantes em que $|\dot{\omega}| \gtrsim \omega^{2}$, em que a freqüência está na vizinhança de seu mínimo (assim como o inflaton) em $t_{j}=j \pi / m, j=1,2, \ldots$, temos que a massa do 
inflaton se anula. A condição adiabática é violada fortemente nesses instante e pode ser reescrita da forma:

$$
\frac{\triangle t / \triangle t_{*}}{\left[k^{2} \triangle t_{*}^{2}+\left(\triangle t / \triangle t_{*}\right)^{2}\right]} \gtrsim 1,
$$

em que consideramos um pequeno intervalo $\triangle t \ll m^{-1}$ na vizinhança de $t_{j}$ e com:

$$
\triangle t_{*} \simeq(g \Phi m)^{-1 / 2}=\frac{1}{m}\left(\frac{g \Phi}{m}\right)^{-1 / 2} .
$$

Segue que a condição adiabática é quebrada somente em pequenos intervalos de tempo $\triangle t \sim \triangle t_{*}$ próximos de $t_{j},\left[t_{j}-\triangle t_{*}, t_{j}+\triangle t_{*}\right]$ e somente para os modos com:

$$
k<k_{*} \simeq \triangle t_{*}^{-1}=\sqrt{g \Phi m}
$$

Podemos ver que a criação de partículas ocorre em um intervalo de tempo da ordem de $\triangle t_{*} \sim \omega^{-1}$, que corresponde a uma oscilação do campo do inflaton, como podemos ver na Figura 7.63 acima, e é consistente com o princípio da incerteza e nos faz ver a origem totalmente quântica dessas perturbações do vácuo.

Determinada a aproximação semi-clássica, vamos agora calcular o número de partículas criadas utilizando o método analítico mencionado acima e utilizado no efeito Schwinger oscilatório. Ou seja, definida a vizinhança dos instantes $t_{j}$ em que ocorre a criação, a equação geral do sistema (7.50) pode ser aproximada por:

$$
\ddot{\chi}_{\mathbf{k}}+\left(k^{2}+m_{\chi}^{2}+g^{2} \Phi^{2}\left(t-t_{j}\right)^{2}\right) \chi_{\mathbf{k}}=0
$$

uma vez que $\left(t-t_{j}\right)$ na vizinhança é pequeno. Nesse método analítico consideramos o espalhamento das funções de onda in e out, dos estados adiabáticos $t_{j-1}$ e $t_{j}$, por potenciais que têm a forma parabólica entre os instantes $t_{j}$. Fazendo uma mudança de variáveis, podemos escrever essa equação na forma:

$$
\frac{d \chi_{\mathbf{k}}^{2}}{d \tau^{2}}+\left(\kappa^{2}+\tau_{j}^{2}\right) \chi_{\mathbf{k}}=0
$$

em que $\kappa \equiv k / k_{*}$ e $\tau_{j} \equiv\left(t-t_{j}\right) / \triangle t_{*}$ (desconsiderando $m_{\chi}$ que é muito pequeno). Essa equação é análoga à equação (5.125), em que o método WKB é utilizado para um campo externo estático. Isso possibilita utilizarmos o método WKB em cada oscilação.

No instante inicial, a solução é o vácuo adiabático, em que $\alpha_{k}=1$ e $\beta_{k}=0$, $\left(\lim _{\tau / \kappa \rightarrow-\infty} \chi_{\mathbf{k}}^{(0)}=\chi_{w k b, \mathbf{k}}\right)$, dada por:

$$
\chi_{w k b, \mathbf{k}}=\frac{1}{\sqrt{\omega}} \exp \left( \pm i \int \omega d t\right)
$$

e, pela aproximação adiabática, no instante após o espalhamento em que a condição WKB 
não é violada a solução é dada por:

$$
\chi_{\mathbf{k}}^{(1)}(t)=\frac{\alpha_{k}^{(0)}}{\sqrt{2 \omega}} \exp \left(-i \int_{0}^{t} \omega d t\right)+\frac{\beta_{k}^{(0)}}{\sqrt{2 \omega}} \exp \left(+i \int_{0}^{t} \omega d t\right),
$$

em que $\alpha_{k}^{(0)}$ e $\beta_{k}^{(0)}$ são os coeficientes de Bogoliubov, com $\left|\alpha_{\mathbf{k}}^{(0)}\right|^{2}-\left|\beta_{\mathbf{k}}^{(0)}\right|^{2}=1$. O rótulo (0) nos coeficientes de Bogoliubov acima denotam que esse é o primeiro espalhamento do campo. Como calculado no Capítulo 5, temos que:

$$
\left|\alpha_{k}\right|^{2}=1+\mathrm{e}^{-\pi \kappa^{2}}, \quad\left|\beta_{k}\right|^{2}=\mathrm{e}^{-\pi \kappa^{2}} .
$$

Dessa forma, nos instantes seguintes em que a condição de adiabaticidade é válida, a solução da equação (7.63), para cada violação WKB antes do espalhamento no potencial no ponto $t_{j}$ é dada por:

$$
\chi_{\mathbf{k}}^{(j)}(t)=\frac{\alpha_{k}^{(j)}}{\sqrt{2 \omega}} \exp \left(-i \int_{0}^{t} \omega d t\right)+\frac{\beta_{k}^{(j)}}{\sqrt{2 \omega}} \exp \left(+i \int_{0}^{t} \omega d t\right),
$$

em que $\alpha_{k}^{j}$ e $\beta_{k}^{j}$ são constantes entre os instantes $t_{j-1}<t<t_{j}$. Após o espalhamento em $t_{j}$, a solução em $t_{j}<t<t_{j+1}$ é dada por:

$$
\chi_{\mathbf{k}}^{(j+1)}(t)=\frac{\alpha_{k}^{(j+1)}}{\sqrt{2 \omega}} \exp \left(-i \int_{0}^{t} \omega d t\right)+\frac{\beta_{k}^{(j+1)}}{\sqrt{2 \omega}} \exp \left(+i \int_{0}^{t} \omega d t\right) .
$$

em que $\alpha_{k}^{(j+1)}$ e $\beta_{k}^{(j+1)}$ são constantes no intervalo $t_{j}<t<t_{j+1}$.

Podemos reescrever essas funções em termo dos coeficientes de Bogoliubov iniciais, $\alpha_{k}^{(0)}$ e $\beta_{k}^{(0)}$, da forma:

$$
\chi_{\mathbf{k}}^{(j+1)}(t)=\alpha_{k}^{(0)} \chi_{\mathbf{k}}^{(j)}+\beta_{k}^{(0)} \chi_{\mathbf{k}}^{(j) *} .
$$

Com isso, podemos relacionar os coeficientes de Bogoliubov para diferentes espalhamentos:

$$
\begin{aligned}
\left(\begin{array}{c}
\alpha_{\mathbf{k}}^{(j+1)} \\
\beta_{\mathbf{k}}^{(j+1)}
\end{array}\right) & =\left(\begin{array}{cc}
\alpha_{\mathbf{k}}^{(0)} & \beta_{\mathbf{k}}^{*(0)} \\
\beta_{\mathbf{k}}^{(0)} & \alpha_{\mathbf{k}}^{*(0)}
\end{array}\right)\left(\begin{array}{c}
\alpha_{\mathbf{k}}^{(j)} \\
\beta_{\mathbf{k}}^{(j)}
\end{array}\right) \\
& =\left(\begin{array}{cc}
\sqrt{1+\mathrm{e}^{-\pi \kappa^{2}}} \mathrm{e}^{i \lambda_{\mathbf{k}}} & i \mathrm{e}^{-(\pi / 2) \kappa^{2}+2 i \Xi_{\mathbf{k}}} \\
-i \mathrm{e}^{-(\pi / 2) \kappa^{2}-2 i \Xi_{\mathbf{k}}} & \sqrt{1+\mathrm{e}^{-\pi \kappa^{2}}} \mathrm{e}^{-i \lambda_{\mathbf{k}}}
\end{array}\right)\left(\begin{array}{c}
\alpha_{\mathbf{k}}^{(j)} \\
\beta_{\mathbf{k}}^{(j)}
\end{array}\right),
\end{aligned}
$$

em que $\Xi_{\mathbf{k}}=2 \int_{0}^{t_{j}} \omega d t+\arg \beta_{\mathbf{k}}^{j}-\arg \alpha_{\mathbf{k}}^{j}$ e $\lambda_{\mathbf{k}}=\arg \Gamma\left(\frac{1+i \kappa^{2}}{2}\right)+\frac{\kappa^{2}}{2}\left(1+\ln \frac{2}{\kappa^{2}}\right)$

O número de partículas criadas para cada espalhamento é dada, em analogia com o feito no Capítulo 5, como se fosse calculado um espalhamento pela aproximação WKB. 
Dessa forma, a densidade de número de partículas criadas após o espalhamento em $t_{j}$ é dado por:

$$
\begin{aligned}
n_{\mathbf{k}}^{j+1} & =\left|\beta_{\mathbf{k}}^{(j+1)}\right|^{2}=\left|\beta_{\mathbf{k}}^{(0)}\right|^{2}+n_{\mathbf{k}}^{j}\left(1+2\left|\beta_{\mathbf{k}}^{(0)}\right|^{2}\right)+\alpha_{\mathbf{k}}^{*(0)} \beta_{\mathbf{k}}^{(j)} \beta_{\mathbf{k}}^{*(0)} \alpha_{\mathbf{k}}^{(j)} \\
& =n_{\mathbf{k}}^{j}+\left|\beta_{\mathbf{k}}^{(0)}\right|^{2}\left(1+2 n_{\mathbf{k}}^{j}\right)+\alpha_{\mathbf{k}}^{*(0)} \beta_{\mathbf{k}}^{(j)} \beta_{\mathbf{k}}^{*(0)} \alpha_{\mathbf{k}}^{(j)} \\
& =n_{\mathbf{k}}^{j}+\mathrm{e}^{-\pi \kappa^{2}}\left(1+2 n_{\mathbf{k}}^{j}\right)-\underbrace{2 \mathrm{e}^{-(\pi / 2) \kappa^{2}} \sqrt{1+e^{-\pi \kappa^{2}}} \sqrt{n_{\mathbf{k}}^{j}\left(1+n_{\mathbf{k}}^{j}\right)} \operatorname{sen} \theta_{\text {total }}^{j}}_{\text {termo defase }},
\end{aligned}
$$

em que:

$$
\theta_{\text {total }}^{j}=\Xi_{\mathbf{k}}-\lambda_{\mathbf{k}}
$$

Podemos ver claramente na expressão para a densidade de partículas criadas que, como no caso do efeito Schwinger oscilatório, temos uma parte que corresponde à criação espontânea de partículas do vácuo, correspondente à $\left|\beta_{\mathbf{k}}^{(0)}\right|^{2}$, uma parte que corresponde à criação estimulada de partículas por outros bósons já criados nos espalhamentos anteriores, $n_{\mathbf{k}}^{j}\left(1+2\left|\beta_{\mathbf{k}}^{(0)}\right|^{2}\right)$ e um termo de fase. Com isso, podemos ver que no pré-aquecimento o mecanismo de ressonância paramétrica larga cria uma grande quantidade de partículas, uma vez que a criação desses escalares é aumentada fortemente pelos escalares criados anteriormente e isso aumenta drasticamente o número de partículas criadas.

Vemos que esse mecanismo é totalmente compatível com o de criação de partículas no efeito Schwinger oscilatório.

A criação estimulada foi considerada em todos os instantes exceto no inicial que foi considerado como sendo vácuo. Contudo, se pensarmos que tínhamos inflação antes do pré-aquecimento, então, como vimos na seção anterior, temos nosso estado inicial no préaquecimento não é vácuo, mas sim um estado adiabático contendo $n_{\mathbf{k}}^{\text {infl }}$ partículas. Dessa forma, a criação de partículas será ainda maior, uma vez que no primeiro espalhamento teremos criado:

$$
n_{\mathbf{k}}^{(1)}=\left|\beta_{\mathbf{k}}^{(0)}\right|^{2}+n_{\mathbf{k}}^{\text {infl }}\left(1+2\left|\beta_{\mathbf{k}}^{(0)}\right|^{2}\right)
$$

No nosso caso em que temos ressonância paramétrica larga sem expansão do universo, sabemos que os parâmetros $q, \kappa^{2}$ e $\lambda_{k}$ são constantes no tempo, ou seja, não variam para cada $j$. Dessa forma, a fase (7.75) é constante para cada $j$, da forma $\theta_{k}^{j}=\theta_{k} . j$, com:

$$
\theta_{k}=\int_{t_{j-1}}^{t_{j}} \omega_{k} d t=\int_{0}^{\pi / m} \omega_{k} d t
$$


em que $\pi / m$ equivale a meio período da oscilação do inflaton localizado entre dois zeros de sua oscilação.

\section{Equivalência entre o método analítico e a resolução numérica da equação de Mathieu}

As previsões obtidas pelo método analítico desenvolvido acima descrevem de maneira precisa os resultados obtidos através da equação de Mathieu para valores altos de $q$, ou seja, para a ressonância no regime largo. Isso pode ser visto pela coincidência das bandas de instabilidade/estabilidade que obtemos pelo método analítico em comparação com a resolução numérica da equação de Mathieu da forma pelos métodos usuais [46].

Para visualizarmos explicitamente as bandas de ressonância obtidas pelo método analítico, temos que determinar o parâmetro $\mu_{k}$, ou expoente característico, diretamente das relações de recorrência obtidas acima. Vamos fazer isso para o regime em que $n_{\mathbf{k}}^{j} \gg 1$, no qual $\left|\alpha_{\mathbf{k}}^{j}\right| \approx\left|\beta_{\mathbf{k}}^{j}\right|$, ou seja, os coeficientes de Bogoliubov se diferenciam somente por uma fase:

$$
\alpha_{\mathbf{k}}^{j}=\frac{1}{\sqrt{2}} \mathrm{e}^{\left(\pi \mu_{\mathbf{k}}+i \theta_{\mathbf{k}}\right) j}, \quad \beta_{\mathbf{k}}^{j}=\frac{1}{\sqrt{2}} \mathrm{e}^{i \vartheta} \mathrm{e}^{\left(\pi \mu_{\mathbf{k}}-i \theta_{\mathbf{k}}\right) j}
$$

Substituindo esses coeficientes em (7.70), obtemos a equação:

$$
\mathrm{e}^{\left(\pi \mu_{\mathbf{k}}+i \theta_{\mathbf{k}}\right)}=\sqrt{1+\mathrm{e}^{-\pi \kappa^{2}}} \mathrm{e}^{-i \lambda_{\mathbf{k}}}+i \mathrm{e}^{-(\pi / 2) \kappa^{2}-i \vartheta} .
$$

Além da solução (7.78), podemos obter outra solução assintótica de (7.70):

$$
\alpha_{\mathbf{k}}^{j}=\frac{1}{\sqrt{2}} \mathrm{e}^{\left(\pi \mu_{\mathbf{k}}+i \theta_{\mathbf{k}}+i \pi\right) j}, \quad \beta_{\mathbf{k}}^{j}=\frac{1}{\sqrt{2}} \mathrm{e}^{i \vartheta} \mathrm{e}^{\left(\pi \mu_{\mathbf{k}}-i \theta_{\mathbf{k}}-i \pi\right) j},
$$

que exibe a condição:

$$
\mathrm{e}^{\left(\pi \mu_{\mathbf{k}}+i \theta_{\mathbf{k}}\right)}=\sqrt{1+\mathrm{e}^{-\pi \kappa^{2}}} \mathrm{e}^{+i \lambda_{\mathbf{k}}}+i \mathrm{e}^{-(\pi / 2) \kappa^{2}-i \vartheta} .
$$

Com as equações (7.79) e (7.81), conseguimos determinar uma equação para $\mu_{\mathbf{k}}$, excluindo a fase $\vartheta$ :

$$
\begin{aligned}
\mathrm{e}^{\pi \mu_{\mathbf{k}}} & =\left|\cos \left(\Xi_{\mathbf{k}}-\lambda_{\mathbf{k}}\right)\right| \sqrt{1+\mathrm{e}^{-\pi \kappa^{2}}} \\
& +\sqrt{\left(1+\mathrm{e}^{-\pi \kappa^{2}}\right) \cos ^{2}\left(\Xi_{\mathbf{k}}-\lambda_{\mathbf{k}}\right)-1}
\end{aligned}
$$

que fornece o número de partículas criadas pelo tempo $t \approx \pi j / m$, pela equação $(7.57)$. 
Na banda de instabilidade $\mu_{\mathbf{k}}$ tem que ser real e a condição para que $k$ esteja na banda de ressonância é dada por:

$$
\left|\operatorname{tg}\left(\Xi_{\mathbf{k}}-\lambda_{\mathbf{k}}\right)\right| \leq \mathrm{e}^{-(\pi / 2) \kappa^{2}}
$$

em que a dependência de $\lambda_{\mathbf{k}}$ com $k$ é dada acima e $\Xi_{\mathbf{k}}$ é dado por, no caso sem expansão do universo e usando que $\kappa^{2} \ll g \Phi / m$ :

$$
\begin{aligned}
\Xi_{\mathbf{k}} & =\int_{0}^{\pi / m} d t \sqrt{k^{2}+g^{2} \phi^{2}(t)} \approx \frac{2 g \Phi}{m}+\frac{\kappa^{2}}{2}\left[\ln \left(\frac{g \Phi}{m \kappa^{2}}\right)+4 \ln 2+1\right] \\
& =4 \sqrt{q}+\frac{k^{2}}{4 \sqrt{q} m^{2}}\left\{\ln \left[4 q\left(\frac{m}{k}\right)^{2}\right]+4 \ln 2+1\right\}
\end{aligned}
$$

Com isso, tendo a dependência em $k$ de $\Xi_{\mathbf{k}}-\lambda_{\mathbf{k}}$, podemos substituir em (7.81), simplificando o termo :

$$
\arg \Gamma\left(\frac{1+i \kappa^{2}}{2}\right) \approx-0,982 \kappa^{2}
$$

para $\kappa^{2} \ll 1$, que fornece:

$$
\Xi_{\mathbf{k}}-\lambda_{\mathbf{k}} \approx 4 \sqrt{q}+\frac{k^{2}}{8 \sqrt{q} m^{2}}(\ln q+9,474)
$$

para obtermos $\mu_{\mathbf{k}}$.

Com esse resultado podemos ver a comparação entre $\mu_{\mathbf{k}}$ obtido pelo método analítico, com $q=(64 \pi / 4)^{2}$, e obtido pela resolução numérica da equação de Mathieu e vemos que esses resultados coincidem, ou seja, suas bandas de instabilidade (ou estabilidade) coincidem (pelo menos em primeira aproximação), como podemos ver na Figura 7.4 retirada de [46]. 


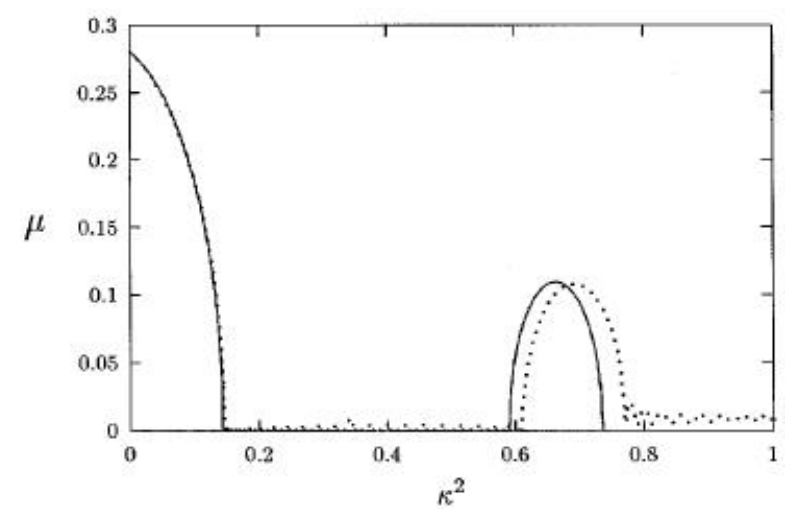

Figura 7.4: Gráfico do expoente característico da equação de Mathieu calculados pelo método analítico (linha cheia) e numericamente (linha tracejada), em que podemos ver duas bandas de instabilidade [46]. Para a primeira banda de instabilidade, os resultados coincidem perfeitamente. Para a outra banda essa coincidência é um pouco pior e pode ser melhorada se a expansão (7.85) for mais precisa.

\section{Formalismo dos estados squeezed}

Analogamente ao feito na Seção 5.2.2.4, para podermos computar o número de partículas criadas em cada espalhamento, vamos reescrever o problema em termos dos parâmetros de squeezing. O procedimento é o mesmo que o feito para o efeito Schwinger oscilatório que é análogo ao feito no caso estático, sendo que utilizaremos a mesma parametrização dos coeficientes de Bogoliubov e $\omega_{\mathbf{k}}^{2}=k^{2}+m_{\chi}^{2}+g^{2} \Phi^{2} \operatorname{sen}^{2}(m t)$. 

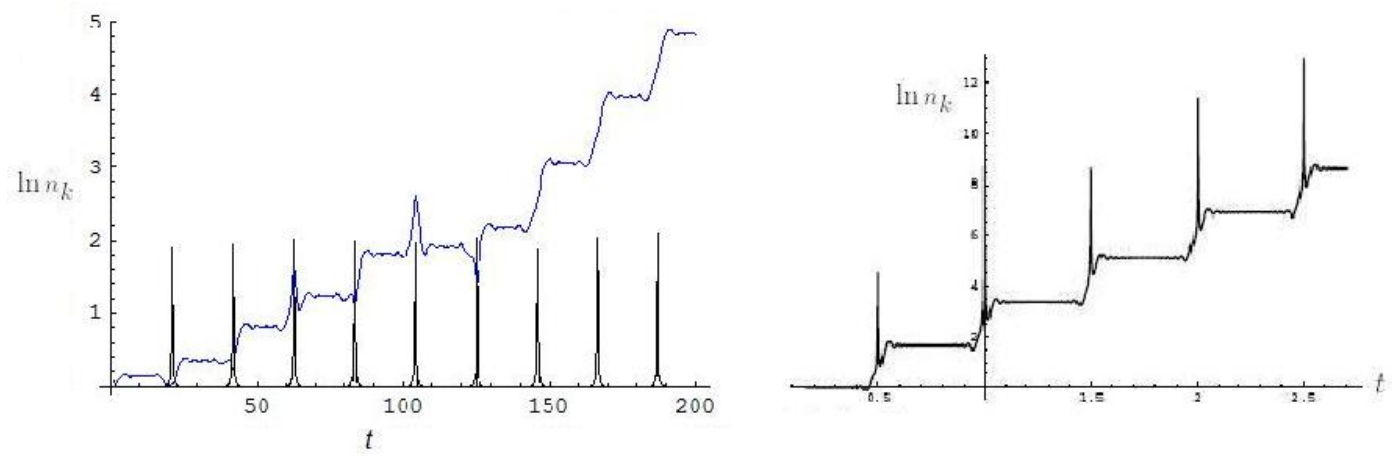

Figura 7.5: No quadro da esquerda temos a simulação feita por nós para o logaritmo do número de partículas criadas pré-aquecimento, sem expansão do universo no regime de ressonância larga. Apresentamos também o parâmetro WKB normalizado por 100 para não atrapalhar na escala do número de partículas. No quadro da direita vemos a simulação feita na referência [46] para o mesmo regime, em que vemos uma maior precisão na simulação.

Através da resolução das equações para os parâmetros de squeeze, calculamos o número de partículas criadas para o pré-aquecimento no regime de ressonância paramétrica larga, ou seja, com $g \Phi>m$. Podemos ver na Figura 7.5, no quadro da esquerda, a simulação feita em que a criação de partículas ocorre entre platôs, como uma função degrau no tempo. No gráfico colocamos também a função WKB para mostrarmos que a criação de partículas ocorre exatamente nos instantes em que a adiabaticidade é violada. No quadro da direita da mesma figura mostramos a simulação do mesmo sistema feita na referência [46], que exibe maior precisão em seus resultados.

Como vimos anteriormente, no caso do pré-aquecimento o número de partículas também cresce em geral, mas pode decrescer em alguns ciclos. Esse resultado aparece naturalmente no formalismo de estados squeezed e depende da fase com que a função de onda (solução) tem antes do espalhamento, uma vez que esses estados a serem espalhados não são estados puros, mas sim estados squeezed.

\subsubsection{Criação de partículas com expansão do universo: ressonância esto- cástica}

Vamos mencionar rapidamente as diferenças no tratamento quando consideramos a expansão do universo para o cálculo da criação de partículas no pré-aquecimento pelo método analítico desenvolvido.

Em um universo em expansão para termos um pré-aquecimento eficiente, é necessário que os valores iniciais do parâmetro $q$ sejam grandes. Isso pode ser visto através da condição para ressonância paramétrica em um universo em expansão, $q^{2} m \gtrsim H$, que para 
nosso modelo se traduz na condição:

$$
g \Phi \gtrsim 2 m\left(\frac{H}{m}\right)^{1 / 4}
$$

em que, como vimos, durante a primeira oscilação do inflaton sua amplitude é dada por (3.52), que fornece um parâmetro de Hubble da ordem de 0,1 m. Dessa forma, para termos um pré-aquecimento eficiente, $\Phi>m / g$.

Como a amplitude do campo escalar decresce muito mais rapidamente que $H^{1 / 4}$, devido à expansão do universo, para que o mecanismo de pré-aquecimento, em que ocorre a transferência de energia do inflaton oscilante para partículas $\chi$ ocorra é necessário que $q_{0} \gtrsim 10^{3}$.

Com isso, para modelos de pré-aquecimento com $q_{0}$ muito grande, vamos estudar o regime de ressonância paramétrica através do método analítico desenvolvido acima.

Partindo da equação de movimento geral para o reaquecimento (7.40), podemos obter uma equação do tipo de um oscilador paramétrico, fazendo a transformação de variáveis $X_{k}=a^{3 / 2}(t) \chi_{k}(t)$ que nos fornece:

$$
\ddot{X}_{k}+\omega_{k}^{2} X_{k}=0
$$

com:

$$
\omega_{k}^{2}=\frac{k^{2}}{a^{2}(t)}+g^{2} \Phi^{2} \operatorname{sen}^{2}(m t)+\Delta,
$$

em que:

$$
\Delta=m_{\chi}^{2}-\frac{3}{4}\left(\frac{\dot{a}}{a}\right)^{2}-\frac{3}{2}\left(\frac{\ddot{a}}{a}\right)-\xi R .
$$

Essa equação tem a mesma forma da equação (7.49), uma vez que o termo $\Delta$ é muito pequeno, pois consideramos $m_{\chi}$ muito pequeno (ou até mesmo nulo) e, logo após a inflação, $H^{2}=(\dot{a} / a)^{2} \sim \ddot{a} / a \ll m^{2}$ e dessa forma podemos desconsiderar o termo $\Delta$. Contudo, temos que considerar nesse caso a dependência em $a(t)$ com cuidado.

Essa equação descreve um oscilador paramétrico com freqüência dependente do tempo e pode ser escrita como uma equação de Mathieu da forma (7.50), com parâmetros dados por:

$$
A_{k}=\frac{(k / a)^{2}}{m^{2}}+2 q, q=\frac{g^{2} \Phi^{2}}{4 m^{2}}
$$

em que $z=m t$. Contudo, temos que ficar atentos quando consideramos a expansão do universo, uma vez que o momento $k$ passa a ser o momento comóvel ou momento que sofre redshift, uma vez que como o universo expande o fator de escala cresce, $k / a$. 
Temos também que o parâmetro $q$ exibe dependência temporal, $q \propto t^{-2} \propto N^{-1}$, uma vez que após a inflação $a(t) \propto t^{2 / 3}$ e $\Phi$ evolui como (3.52). Portanto, se tivermos o regime de ressonância larga $(q \gg 1$ e muito grande), esse parâmetro varia fortemente em apenas algumas oscilações, fazendo com que o conceito da análise das bandas de estabilidade/instabilidade do gráfico de Mathieu não possa ser aplicado. Mesmo com todas essas limitações, tendo a mesma equação de Mathieu descrevendo a ressonância paramétrica, podemos aplicar o método analítico apresentado acima no caso com expansão do universo de maneira análoga, sendo que o número total de partículas criadas agora é dado por:

$$
n_{\chi}(t)=\frac{1}{(2 \pi a)^{3}} \int d^{3} k n_{k}(t)
$$

sendo que a a matriz dos coeficientes de Bogoliubov é a mesma que em (7.70) e, dessa forma, $n_{k}(t)$ é dado por relações de recorrência (7.74).

Podemos diferenciar, para o caso com expansão do universo, a fase (7.75) acumulada entre dois zeros da oscilação do inflaton:

$$
\begin{aligned}
\theta_{k}^{j} & =\int_{t_{j-1}}^{t_{j}} \omega_{k} d t \approx \frac{2 g \Phi}{m}+\frac{\kappa^{2}}{2}\left[\ln \left(\frac{g \Phi}{m \kappa^{2}}\right)+4 \ln 2+1\right] \\
& \approx \frac{g m_{p l}}{5 m j}+\mathcal{O}\left(\kappa^{2}\right) .
\end{aligned}
$$

Como estamos considerando $q_{0}$ muito grande, a variação dessa fase entre dois espalhamentos é dada por:

$$
\delta \theta_{k} \simeq \frac{\sqrt{q}}{2 N^{2}} .
$$

Ou seja, para os momentos importantes para o sistema, essa variação é muito maior que $\pi$ e dessa forma todas as fases em (7.74) são consideradas números aleatórios para as primeiras:

$$
N_{\text {estocástica }} \simeq \frac{q^{1 / 4}}{\sqrt{2 \pi}},
$$

oscilações. Os modos exibem um comportamento caótico durante esse período.

Esse comportamento estocástico da fase simplifica a análise, uma vez que ela é aleatória e cada valor é independente do anterior. Podemos expressar a fase total, $\theta_{\text {total }}^{j}$, em função da fase aleatória $\theta_{k}^{j}$ e, assim, considerando a fase total aleatória, de (7.74), podemos verificar que o número de partículas criadas é dado por: 


$$
n_{\mathbf{k}}^{j+1} \approx n_{\mathbf{k}}^{j}\left(1+2\left|\beta_{\mathbf{k}}^{(0)}\right|^{2}\right)-2 \operatorname{sen} \hat{\theta} \mathrm{e}^{-(\pi / 2) \kappa_{j}^{2}} \sqrt{1+e^{-\pi \kappa_{j}^{2}}} n_{\mathbf{k}}^{j}
$$

sendo que esse número de partículas pode aumentar ou diminuir devido à fase aleatória. Já havíamos observado esse comportamento no caso sem expansão do universo, devido ao formalismo squeezed, contudo com expansão do universo temos também a oscilação devido ao formalismo associada à aleatoriedade da fase $i n, n_{\mathrm{k}}^{j}$, que pode fazer com que o número de partículas aumente ou diminua.

Através desse método analítico podemos ver, como no caso anterior, que a criação de partículas ocorre em degraus, crescendo e decrescendo entre os instantes em que a adiabaticidade é violada, mas em geral crescendo, como podemos ver na Figura 7.6. Esse comportamento também é análogo ao de criação de partículas no efeito Schwinger oscilatório, apesar de não implicar na existência de uma estrutura de bandas estabilidade/instabilidade completamente separadas.

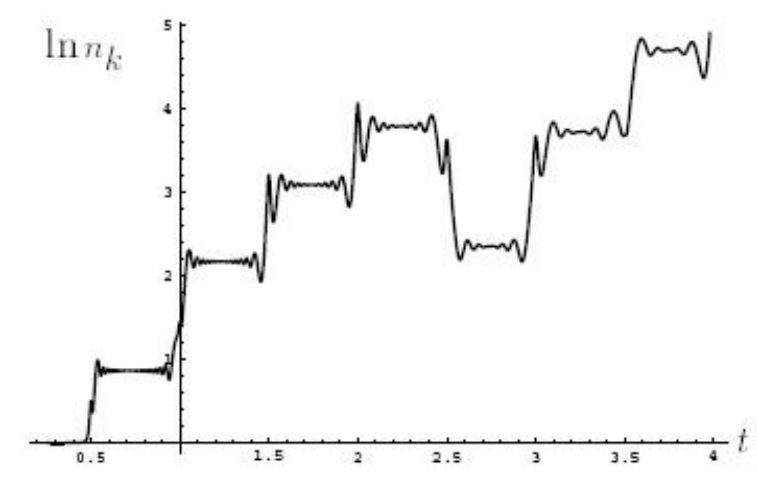

Figura 7.6: Figura retirada de [46] que mostra o logaritmo do número de partículas criadas durante o pré-aquecimento considerando a expansão do universo, devido à ressonância estocástica.

Com isso, a ressonância estocástica é mais ampla e mais estável que a ressonância paramétrica larga vista anteriormente, fazendo com que seja menos sensível a mecanismos responsáveis pelo fim desse regime, fazendo com que a criação de partículas seja mais eficiente.

O mecanismo de ressonância estocástica acaba após completadas $N_{\text {estocastica }}$ oscilações, entrando em um mecanismo de ressonância estreita, assim como no caso sem expansão do universo.

Em nosso estudo, ignoramos efeitos de backreaction. Essa aproximação é válida para os estágios iniciais do pré-aquecimento em que a ressonância é larga. Se o pré-aquecimento apresenta um valor inicial do parâmetro $q \lesssim 10^{3}$, sua ressonância se torna estreita e o 
pré-aquecimento acaba antes que efeitos de backreaction comecem a se tornar importantes. Caso contrário, o tratamento do pré-aquecimento ignorando efeitos de backreaction somente é uma boa aproximação, para seus estágios iniciais, em que a criação de partículas é mais significativa, sendo necessária outra análise, considerando backreaction, desse segundo estágio do pré-aquecimento. Dessa forma, nossa análise é válida para os estágios iniciais do pré-aquecimento. Para ver mais sobre o estudo do pré-aquecimento considerando efeitos de backreaction, assim como reespalhamentos, ver referências [46, 134]. 


\section{Capítulo 8}

\section{Conclusão}

Nessa dissertação analisamos efeitos associados a flutuação do vácuo quântico, especificamente a possibilidade de se fazer uma analogia entre os efeitos Schwinger estático e a inflação e entre o efeito Schwinger oscilatório e o pré-aquecimento, como uma forma de possibilitar a verificação desses efeitos quânticos de criação de partículas no universo jovem.

O efeito Schwinger, que consiste em um sistema quântico submetido a um campo externo eletromagnético clássico, é descrito com um oscilador paramétrico com dependência temporal proporcional a $t^{2}$. Ele foi descrito originalmente via o formalismo das Lagrangeanas efetivas, que foi reproduzido em linguagem mais moderna no Apêndice A dessa dissertação; via teoria de espalhamento, com o intuito de obtenção dos limites observacionais do efeito e via transformadas de Bogoliubov para a obtenção do número de partículas criadas. Através desse último método, foi calculado o número de partículas criadas pela aproximação semi-clássica, ou adiabática, em que as partículas são criadas entre estados iniciais e finais adiabáticos.

Mostramos que é possível obtermos a mesma descrição para o período inflacionário. Durante esse período o universo se expande rapidamente e isso faz com que surjam perturbações do vácuo de origem quântica. Essas perturbações são a criação de partículas que inomogeneizam o universo. Esse efeito de criação de partículas na inflação devido à expansão do universo pode ser escrita, somando uma derivada total na ação de EinsteinHilbert que descreve o inflaton em um espaço curvo que se expande exponencialmente, como a ação do inflaton quântico sujeito a um potencial externo gravitacional, assim como é descrito o efeito Schwinger. A dependência temporal da freqüência do oscilador paramétrico que descreve a inflação difere da do efeito Schwinger estático apresentando uma dependência proporcional a $1 / \eta^{2}$, apesar desses dois efeitos apresentarem a mesma física. Dessa forma, é possível aplicar o mesmo método que no caso do efeito Schwinger estático para a obtenção do número de partículas criadas.

No caso inflacionário, o estado inicial é o vácuo de Bunch-Davies, que emerge naturalmente da validade da aproximação adiabática no limite de comprimentos de onda 
menores que o raio de Hubble. Contudo, para comprimentos de onda maiores que o raio de Hubble, a aproximação WKB não é válida e não é possível definir um estado adiabático final (out). Assim, para ser possível aplicarmos a aproximação WKB, consideramos a evolução do universo até o fim da inflação e começo do reaquecimento, quando o universo se comporta como dominado por matéria e o estado adiabático é bem definido e os modos reentram o raio de Hubble. Definidos os estados inicial e final adiabáticos, é possível, então, calcular o número de partículas criadas na inflação pelo mesmo método feito no efeito Schwinger estático.

Esse resultado é importante uma vez que como é impossível termos um experimento que refaça o período inflacionário, ao vermos a analogia entre os dois fenômenos, temos que a detecção experimental do efeito Schwinger estático seria uma corroboração da existência desse mecanismo de criação de partículas na inflação. Como vimos, esse efeito ainda não foi detectado, uma vez que são necessários campos estáticos da ordem de $E_{\text {crit }} \sim$ $1.3 \times 10^{18} \mathrm{~V} / \mathrm{m}$, e esses ainda são inatingíveis experimentalmente. A detecção do efeito Schwinger estático é importante também pois seria a primeira detecção de um fenômeno não-perturbativo na QED.

A segunda analogia feita nessa dissertação foi entre o efeito Schwinger oscilatório e a criação de partículas no pré-aquecimento. O efeito Schwinger oscilatório é análogo ao estático, mas com um campo eletromagnético externo oscilatório. Esse mecanismo foi descrito na literatura em termos da teoria de espalhamento, transformadas de Bogoliubov, entre outras descrições. Aqui apresentamos o efeito via teoria de espalhamento, baseada na descrição em [74] e via transformadas de Bogoliubov. Contudo, obtivemos uma descrição diferente para a descrição via transformadas de Bogoliubov do efeito Schwinger oscilatório. Mostramos que esse efeito é escrito também como um oscilador paramétrico com dependência oscilatória de seus termos. Essa equação representa uma equação de Hill, para o caso do campo escalar complexo, que pode ser reduzida a uma equação de Mathieu considerando a condição de ortogonalidade $k_{3} A_{3}=0$. Se modelarmos o efeito Schwinger oscilatório com um campo escalar (não complexo), podemos obter uma descrição do efeito por uma equação de Mathieu, somando uma derivada total na Lagrangeana que descreve o efeito, eliminando o termo linear no potencial externo.

Vimos que a condição de criação no regime não-perturbativo, obtida via teoria de espalhamento, coincide com o regime de criação de ressonância larga, que é o mais eficiente. Para resolvermos essa equação de Mathieu do efeito Schwinger oscilatório, foi adotado um método analítico em que, nos instantes em que o campo eletromagnético externo passa pelo mínimo de seu potencial, podemos escrever nossa equação que descreve o fenômeno como em presença de um potencial parabólico, análogo ao caso estático. Aplicamos, assim, o método semi-clássico para a obtenção da criação de partículas entre cada um desses instantes em que o campo é nulo e os estados são adiabáticos. A criação de partículas ocorre em "degraus", sendo que a criação nos instantes anteriores amplificam as próximas 
criações por um fator de Bose. Isso pôde ser visto através do formalismo dos estados squeezed, que reflete o caráter squeezed dos estados desse efeito responsáveis pela criação de partículas .

Mostramos que esse efeito é análogo à criação de partículas no pré-aquecimento, que é o estágio inicial do reaquecimento, período no qual quase todas as partículas elementares existentes hoje (ou suas precursoras) foram criadas. No reaquecimento, que ocorre após o período inflacionário, o inflaton que atingiu o fundo de seu potencial passa a oscilar em torno dele. Esse período pode ser descrito como um campo escalar na presença do inflaton oscilante, em que se considerarmos o caso em que não há expansão do universo e não consideramos efeito de backreaction, podemos descrevê-lo através de uma equação de Mathieu, da mesma forma que o efeito Schwinger oscilatório. Dessa forma, novamente foi possível fazer a analogia entre o efeito eletromagnético e o gravitacional, que apresentam a mesma dinâmica.

Focamos nossa análise ao período em que a criação de partículas é mais eficiente no pré-aquecimento, que ocorre quando a ressonância paramétrica é larga, assim como no efeito Schwinger estático. Utilizamos o mesmo método analítico para a resolução desse sistema, em que a criação de partículas ocorre entre os instantes em que o inflaton passa pelo mínimo de seu potencial, descrito também como um oscilador paramétrico com potencial parabólico. O método semi-clássico foi utilizado para obter a criação de partículas em "degraus", como no efeito Schwinger oscilatório, em que a mesma amplificação foi observada.

Em ambos os casos o número de partículas cresce em geral, apesar de que pode decrescer em alguns períodos, dependendo da fase inicial do estado. Contudo, se no caso do pré-aquecimento for considerada a expansão do universo, a mesma análise pelo método analítico para a criação de partículas pode ser feita. Esse sistema descreve uma oscilação estocástica, em que a fase inicial é aleatória, então o crescimento/decrescimento em cada "degrau" é também aleatório, apesar de em geral crescer.

Uma vez que esse efeitos são análogos, novamente a verificação experimental do efeito Schwinger oscilatório seria uma importante verificação da validade da hipótese do mecanismo de criação de partículas no pré-aquecimento. Contudo, apesar de campos elétricos oscilatórios serem mais fáceis de serem obtido experimentalmente, a comprovação do efeito eletromagnético ainda não foi possível.

Mostramos que uma esperança no sentido da verificação desse efeito está no efeito análogo chamado efeito Casimir dinâmico, uma vez que esse efeito oscilatório, um análogo ao conhecido efeito Casimir, que consiste na criação de partículas do vácuo pela oscilação da(s) parede(s) de um capacitor ou se de uma cavidade pode ser descrito como um campo na presença de um potencial externo, assim como os efeito Schwinger oscilatório e o préaquecimento. Um experimento baseado nesse efeito, o $M I R$, está em fase final ajustes e deve nos fornecer resultados em 2010. 


\section{Referências Bibliográficas}

[1] A. Einstein, Zur Quantentheorie der Strahlung (Alemanha, 1917), http:/ / nausikaa2.rz-berlin.mpg.de/di...g/ $\& p n=2 \& w s=1.5$.

[2] A. Einstein, em "The Principle of Relativity", notas por A. Sommerfeld (Dover, London 1953).

[3] E. Hubble, Proc. Nat. Acad. Sci. 15, 168 (1929).

[4] R. A. Alpher, H. Bethe e G. Gamow, Phys. Rev. 73, 803 (1948).

[5] G. Gamow, Nature 162, 680 (1948).

[6] R. A. Alpher e R. Herman, Nature 162, 774 (1948).

[7] A. A. Penzias e R. W. Wilson, Astrophys. J. 142, 419 (1965).

[8] J. C. Mather et al., Astrophys. J. 354, L37 (1990).

[9] http://lambda.gsfc.nasa.gov/product/cobe/.

[10] A. H. Guth, Phys. Rev. D23, 347 (1981).

[11] A. A. Starobinsky, Phys. Lett. B91, 99 (1980).

[12] S. Perlmutter et al., Astrophys. J. 517, 565 (1999).

[13] A. G. Riess et al., Astron. J. 116, 1009 (1998).

[14] A. G. Riess et al., Astrophys. J. 560, 49 (2001).

[15] A. H. Jaffe et al., Phys. Rev. Lett. 86, 3475 (2001).

[16] H. B. Callen, Thermodynamics and an Introduction to Thermostatistics (Wiley, 1985).

[17] http://www.astro.iag.usp.br/ ronaldo/.

[18] W. L. Freedman et al., Astrophys. J. 553, 47 (2001). 
[19] S. Weinberg, (2000), astro-ph/0005265.

[20] G. Gazzola, M. C. Nemes and W. F. Wreszinski, (2008), gr-qc/0808.1267.

[21] http://map.gsfc.nasa.gov/.

[22] WMAP, C. L. Bennett et al., Astrophys. J. Suppl. 148, 1 (2003), astro-ph/0302207.

[23] WMAP, D. N. Spergel et al., Astrophys. J. Suppl. 170, 377 (2007), astro$\mathrm{ph} / 0603449$.

[24] WMAP, E. Komatsu et al., (2008), astro-ph/0803.0547.

[25] WMAP, G. Hinshaw et al., (2008), astro-ph/0803.0732.

[26] http://www.sdss.org/.

[27] J. Yadav, S. Bharadwaj, B. Pandey e T. R. Seshadri, Mon. Not. Roy. Astron. Soc. 364, 601 (2005), astro-ph/0504315.

[28] H. Weyl, Raum-Zeit-Materie (5th ed.), Springer, Berlin (1923).

[29] S. Weinberg, Gravitation and Cosmology (Wiley, 1972).

[30] S. Weinberg, Cosmology (Oxford University Press, USA, 2008).

[31] V. Mukhanov, Physical Foundations of Cosmology (Cambridge University Press, USA, 2005).

[32] S. Dodelson, Modern Cosmology (Academic Press, 2003).

[33] A. D. Linde, Phys. Lett. B108, 389 (1982).

[34] A. Albrecht and P. J. Steinhardt, Phys. Rev. Lett. 48, 1220 (1982).

[35] V. F. Mukhanov and G. V. Chibisov, JETP Lett. 33, 532 (1981).

[36] A. Riotto, (2002), hep-ph/0210162v1.

[37] T. S. Pereira, C. Pitrou e J.-P. Uzan, JCAP 0709, 006 (2007), astro-ph/0707.0736 .

[38] C. Pitrou, T. S. Pereira, and J.-P. Uzan, JCAP 0804, 004 (2008), astro$\mathrm{ph} / 0801.3596$.

[39] A. D. Linde, Phys. Lett. B 129, 177 (1983).

[40] A. Albrecht, P. J. Steinhardt, M. S. Turner e F. Wilczek, Phys. Rev. Lett. 48, 1437 (1982). 
[41] A. D. Dolgov e A. D. Linde, Phys. Lett. B116, 329 (1982).

[42] L. F. Abbott, E. Fahri e M. B. Wise, Phys. Lett. B117, 29 (1982).

[43] D. V. Nanopoulos, K. A. Olive e M. Srednicki, Phys. Lett. B127, 30 (1983).

[44] A. D. Dolgov, Phys. Rept. 222, 309 (1992).

[45] L. Kofman, A. D. Linde e A. A. Starobinsky, Phys. Rev. Lett. 73, 3195 (1994), hep-th/9405187.

[46] L. Kofman, A. D. Linde e A. A. Starobinsky, Phys. Rev. D56, 3258 (1997).

[47] J. M. Bardeen, Phys. Rev. D22, 1882 (1980).

[48] R. Brandenberger, (2007), hep-th/0701157v1.

[49] V. F. Mukhanov, H. A. Feldman e R. H. Brandenberger, Phys. Rept. 215, 203 (1992).

[50] M. Abramowitz e I. A. Stegun, Handbook of Mathematical Functions with Formulas, Graphs, and Mathematical Tables (Dover Publications, USA, 1965).

[51] G. Arfken e H. Weber, Mathematical Methods for Physicists (Academic Press, USA, 2000).

[52] J. M. Bardeen, P. J. Steinhardt and M. S. Turner, Phys. Rev. D28, 679 (1983).

[53] J. Martin and D. J. Schwarz, Phys. Rev. D57, 3302 (1998).

[54] D. Wands, A. Malik, D. H. Lyth and A. R. Liddle, Phys. Rev. D62, 043527 (2000).

[55] L. R. Abramo, (1997), 9709049v4.

[56] V. N. Lukash, Sov. Phys. JETP 52, 807 (1980).

[57] D. H. Lyth, Phys. Rev. D31, 1792 (1985).

[58] S. Weinberg, Phys. Rev. D67, 123504 (2003), astro-ph/0302326.

[59] W. B. Lin, X. H. Meng and X. M. Zhang, Phys. Rev. D61, 121301 (2000), hep$\mathrm{ph} / 9912510$.

[60] A. Riotto, (2002), 0210162v1.

[61] R. Arnowitt, S. Deser, and C. W. Misner, (1962), gr-qc/0405109; em: Gravitation: An Introduction to Current Research (Wiley, New York, 1962). 
[62] N. D. Birrell e P. C. W. Davies, Quantum Fields in Curved Space (Cambridge Univ. Press, USA, 1982).

[63] D. Langlois, (2004), hep-th/0405053.

[64] R. E. Langer, Phys. Rev. 51, 669 (1937).

[65] R. Casadio, F. Finelli, M. Luzzi e G. Venturi, Phys. Rev. D 72, 103516 (2005), gr-qc/0510103.

[66] L. A. Kofman, astro- ph/9605155.

[67] L.F. Abbott e D.D. Harari, Nucl. Phys. B 264, 487 (1986).

[68] E. A. Calzetta, A. Kandus e F. D. Mazzitelli, Phys. Rev. D 57, 7139 (1998).

[69] F. Sauter, Z. Physik. 69742, 1931.

[70] W. Heisenberg and H. Euler, Z. Phys. 98, 714732, 1936.

[71] V. Weisskopf, The electrodynamics of the vacuum based on the quantum theory of the electron. In *Miller, A.I.: Early quantum electrodynamics* 206-226.

[72] J. Schwinger, Phys. Rev. 82, 664 (1951).

[73] L. V. Keldysh, Sov. Phys. JETP 20, 1307 (1965).

[74] E. Brezin and C. Itzykson, Phys. Rev. D2: 1191 (1970).

[75] C. Itzykson and J.-B. Zuber, Quantum Field Theory (McGraw-Hill, New York 1980).

[76] A. Zee, Quantum Field Theory in a Nutshell (Princeton University Press, Princeton 2003).

[77] S. Weinberg, The Quantum Theory of Fields, Vols. 1-3 (Cambridge University Press, 2000).

[78] G. T. Moore, J. Math. Phys. 11, 2679 (1970).

[79] S. A. Fulling e P. C. W. Davies, Proc. Roy. Soc. London A 348, 393 (1976).

[80] V. V. Dodonov, Concepts of Physics, Vol. IV, No. 3 (2007).

[81] V. V. Dodonov e A. V. Dodonov, J. Russian Laser Research 26 (6), 445 (2005).

[82] R. Marcus, http://www.unige.ch/cyberdocuments/theses2007/RuserM/these.pdf, tese de doutorado (2007). 
[83] C. Braggio et al., Europhys. Lett., 70 (6),754 (2005).

[84] J. Martin, (2007), hep-th/0704.3540v1.

[85] G. Mahajan e T. Padmanabhan, (2007), gr-qc/0708.1233.

[86] G. Mahajan e T. Padmanabhan, (2007), gr-qc/0708.1237.

[87] L. Sriramkumar e T. Padmanabhan, (1999), gr-qc/9903054v3.

[88] C. Kiefer e D. Polarski, Annalen Phys. 7, 137 (1998), gr-qc/9805014v2.

[89] W. E. Lamb e R. C. Retherford, Phys. Rev. 79, 549 (1950).

[90] S. G. Karshenboim, Phys. Rep. 422, 1 (2005), hep-ph/0509010v1.

[91] G. Gabrielse, D. Hanneke, T. Kinoshita, M. Nio e B. C. Odom, Phys. Rev. Lett. 97, 030802 (2006) [Erratum-ibid. 99, 039902 (2007)].

[92] T. Kinoshita e M. Nio, Phys. Rev. D73, 013003 (2006), hep-ph/0507249.

[93] T. Aoyama, M. Hayakawa, T. Kinoshita e M. Nio, Phys. Rev. D77, 053012 (2008) hep-ph/0712.2607 .

[94] Sh. Zh. Akhmadaliev et al., Phys. Rev. C58, 2844 (1998).

[95] C. Bula et al. [E144 Collaboration], Phys. Rev. Lett. 76, 3116 (1996).

[96] H. B. G. Casimir, Indag. Math. 10, 261 (1948) [Kon. Ned. Akad. Wetensch. Proc. 51, 793 (1948)].

[97] R. L. Jaffe, Phys. Rev. D72, 021301 (2005), hep-th/0503158; T. Emig and R. L. Jaffe, J. Phys. A41, 164001 (2008), quant-ph/0710.5104 .

[98] M. Bordag, U. Mohideen e V. M. Mostepanenko, Phys. Rept. 353, 1 (2001) quant$\mathrm{ph} / 0106045$.

[99] T. Damour, Klein paradox and vacuum polarization, em Proceedings of The First Marcel Grossmann Meeting on General Relativity, Amsterdam, 1975.

[100] A. I. Nikishov, Soviet Physics JETP 30(4), 660 (1970).

[101] L. Sriramkumar e T. Padmanabhan, gr-qc/9903054.

[102] L. Sriramkumar, Mod. Phys. Lett. A. 14, 1869 (1999).

[103] J. Hallin e P. Liljenberg, Phys. Rev. D52 (2), 1150 (1995).

[104] C. Keifer e A. Wipf, Ann. Phys. 236, 241 (1994). 
[105] L. Sriramkumar e T. Padmanabhan, Phys. Rev. D54 (12), 7599 (1996).

[106] G. V. Dunne, (2008), hep-th/0812.3163v1.

[107] A. F. R. De Toledo Piza, Mecânica Quântica (EDUSP, Brasil, 2003).

[108] N. N. Bogoliubov, JETP 34 (1), 58 (1958).

[109] J. Blaizot e G. Ripka, Quantum Theory of Finite Systems (The MIT Press, USA, 1985).

[110] K. Srinivasan e T. Padmanabhan, (1998), gr-qc/9807064v1.

[111] M. F. Atiyah e I. G. MacDonald, Introduction to Commutative Algebra, (Westview Press, 1969).

[112] S. P. Kim e F. C. Khanna, (2003), quant-ph/0308053v1.

[113] J. Hallin e P. Liljenberg, Phys. Rev. D52, 1150 (1995).

[114] T. Arimitsu e H. Umezawa, Prog. Theor. Phys. 74, 429 (1985).

[115] T. Arimitsu e H. Umezawa, Prog. Theor. Phys. 77, 32 (1987).

[116] T. Arimitsu e H. Umezawa, Prog. Theor. Phys. 77, 53 (1988).

[117] T. Arimitsu, M. Guida e H. Umezawa, Physica A 148, 1 (1988);

[118] Y. Yamanaka, H. Umezawa, K. Nakamura e T. Arimitsu, Int. Mod. Phys. A 9, 1153 (1994);

[119] P.A. Henning and H. Umezawa, Nucl. Phys. B 417, 463 (1994).

[120] J. Schwinger, Phys. Rev. 93, 615 (1954).

[121] M. S.Marinov e V. S. Popov, Sov. J. Nucl. Phys. 16, 449 (1973).

[122] http://xfel.desy.de/.

[123] N.B. Narozhnyi and A.I. Nikishov, Yad. Fiz. 11, 1072 (1970) [Sov. J. Nucl. Phys. 11, $596(1970)]$.

[124] V. S. Popov, Sov. JETP 34, 709 (1972).

[125] A. Munier, J. R. Burgan, M. Feix e E. Fijalkow. J. Math. Phys. 22, 1219 (1980).

[126] J. Schwinger, Proc. Natl. Acad. Sci. 90, 2105 (1993).

[127] A. Agnesi et al., J. Phys. A: Math. Theor. 41, 164024 (2008) . 
[128] http://www.extreme-light-infrastructure.eu/.

[129] http://www.gsi.de/informationen/wti/library/06.pdf.

[130] R. Schützhold, H. Gies e G. Dunne, Phys. Rev. Lett. 101, 130404 (2008).

[131] T. Harko e K. S. Cheng, (2006), astro-ph/0601564v1.

[132] R. Ruffini, L. Vitagliano e S. Xue, Phys. Lett. B559, 12 (2003).

[133] N. W. McLachlan, Theory and application of Mathieu functions (Dover Publications, USA,1964).

[134] S. Khlebnikov e I. Tkachev, Phys. Lett. B390, 80 (1977).

[135] H. B. G. Casimir e D. Polder, Phys. Rev 73, 360 (1948).

[136] G. T. Moore. Math. Phys. 11, 2679 (1970).

[137] C. K. Law. Phys. Rev. A49, 433 (1994).

[138] V. V. Dodonov, A. B. Klimov. Phys. Rev. A53, 2664 (1996).

[139] V. V. Dodonov and A. B. Klimov e D. E. Nikonov, J. Math. Phys. 34, 2742 (1993).

[140] C. K. Law, Phys. Rev. Lett. 73, 1931 (1994).

[141] C. K. Cole and W. C. Schieve, Phys. Rev. A. 52, 4405 (1995).

[142] C. K. Cole and W. C. Schieve, Phys. Rev. A. 64, 023813 (2001).

[143] P. A. Maia Neto, J. Phys. A: Math. Gen. 27, 2167 (1994).

[144] D. A. R. Dalvit, F. D. Mazzitelli e X. Orsi Millán, J. Phys. A: Math. Gen. 39, 6261 (2006).

[145] W. G. Unruh, Phys. Rev. D14, 870 (1976).

[146] G. T. Moore, J. Math. Phys. 11, 2679 (1970).

[147] V. V. Dodonov e A. B. Klimov, Phys. Rev. A. 53, 2664 (1996).

[148] I.S. Gerstein and R. Jackiw, Phys. Rev. 181 (1969).

[149] D.L. Burke et al., Phys. Rev. Lett. 79, 1626 (1997).

[150] A.C. Melissinos, in: P. Chen (Ed.), Quantum Aspects of Beam Physics, Proc. 15th Advanced ICFA Beam Dynamics Workshop, Monterey, CA, 4-9 January 1998, World Scientific, Singapore, 1998, p. 564. 
[151] C. Bamber et al., Phys. Rev. D 60, 092004 (1999).

[152] R. Runi, in Proceedings of the 34th COSPAR Scientic Assembly - Houston, TX, (Elsevier, USA, 2003).

[153] V. S. Popov, Phys. Lett. A298 (2-3), 83 (2002).

[154] R. Alkofer, M. B. Hecht, C. D. Roberts, S. M. Schmidt e D. V. Vinnik, Phys. Rev. Lett. 87, 193902 (2001).

[155] M. Crocce, D. A. R. Dalvit e F. D. Mazzitelli, Phys. Rev. A. 64, 013808 (2001), quant-ph/0012040.

[156] M. Crocce, D. A. R. Dalvit e F. D. Mazzitelli, Phys. Rev. A. 66, 033811 (2002), quant-ph/0205104.

[157] S. K. Lamoreaux, Phys. Rev. Lett. 78, 5 (1997).

[158] S. K. Lamoreaux, Phys. Rev. Lett 81, 5475 (1997).

[159] U. Mohideen and A. Roy, Phys. Rev. Lett. 81, 4549 (1998), physics/9805038.

[160] C. Braggio et al., (2004), quant-ph/0411085v1.

[161] W. Kim, J. H. Brownell e R. Onofrio, Phys. Rev. Lett. 96, 200402 (2006).

[162] V. V. Dodonov e A. V. Dodonov, J. Phys. B39, S749 (2006).

[163] E. Yablonovitch E., Phys. Rev. Lett. 62, 1742 (1989).

[164] Y. E. Lozovik, V. G. Tsvetus e E. A. Vinogradov, JETP Lett. 61, 723 (1995);

[165] Y. E. Lozovik, V. G. Tsvetus e E. A. Vinogradov, Phys. Scr. 52, 184 (1995).

[166] A. Agnesi et al., J. Phys.: Conference Series 161, 012028 (2009).

[167] T. Padmanabhan, Pramana J. Phys. 37 (3), 179 (1991). 


\section{Apêndice A}

\section{Dinâmica via formalismo da Lagrangeana efetiva (ou da ação efetiva)}

Um campo clássico externo pode produzir partículas (em um campo quântico) devido a efeitos quânticos do vácuo. Isso significa que a dinâmica do campo eletromagnético contém correções quânticas. Entre essas correções temos efeitos de absorção e efeitos de dispersão. Essas correções quânticas do sistema são computadas em um Lagrangeana efetiva, em que sua parte real descreve efeitos de dispersão e sua parte imaginária efeitos de absorção. Assim, o sistema de um campo quântico (escalar ou fermiônico) na presença de um campo clássico elétrico pode ser descrito em termos da Lagrangeana efetiva, sendo que a parte imaginária da Lagrangeana efetiva nos fornece a criação de partículas, como vamos ver.

Vamos aqui calcular o efeito Schwinger no formalismo da Lagrangeana efetiva. Como o campo magnético não cria partículas, em nossa descrição iremos apenas considerar um campo elétrico uniforme. Vamos calcular a Lagrangeana efetiva do problema e, em seguida, a probabilidade por unidade de volume e tempo de não emitir pares, para bósons e férmions.

Consideremos a densidade Lagrangeana descrita em 5.3 com o potencial vetor $A_{\mu}$, para o campo escalar complexo. A teoria quântica completa do sistema pode ser descrita pelo kernel:

$$
K=\int \mathcal{D} A_{\mu} \mathcal{D} \phi \mathcal{D} \phi^{*} \exp \left[i \int \mathcal{L} d t d \mathbf{x}\right],
$$

que também pode ser entendida como a geradora da função de Green:

$$
G\left(x, x^{\prime}\right)=\left\langle x|G| x^{\prime}\right\rangle=\int_{0}^{\infty} d s K(x, x, s)
$$


em analogia com Schwinger. Assim, como a função de Green de um sistema descreve a transição de um estado para outro, temos que o kernel descreve bem a interação que ocorre entre os estados.

A Lagrangeana efetiva, e conseqüentemente a ação efetiva, $W_{\text {eff }}$, podem ser obtidas integrando no campo escalar:

$$
\begin{aligned}
\exp \left(i W S_{\text {eff }}\right) & =\exp \left[i \int d t d \mathbf{x} \mathcal{L}_{\text {eff }}\right] \\
& =\int \mathcal{D} \phi \mathcal{D} \phi^{*} \exp \left(i \int d^{4} x \mathcal{L}\left(\phi, A_{\mu}\right)\right) .
\end{aligned}
$$

Ou seja, para determinarmos a Lagrangeana efetiva temos que calcular a integral de tragetória sobre $\phi$ e $\phi^{*}$ no campo externo eletromagnético. Contudo, essa tarefa é muito difícil para potenciais eletromagnéticos arbitrários. Assim, para possibilitar nossa tarefa, limitamos nosso caso a um campo puramente elétrico e estático, em que o potencial tem dependência temporal, $A_{\mu}=A_{\mu}(t)$.

Portanto, a Lagrangeana efetiva pode ser expressa como:

$$
\exp \left(i \int d^{4} x \mathcal{L}_{e f f}\left(A_{\mu}\right)\right)=\int \mathcal{D} \phi \mathcal{D} \phi^{*} \times \exp \left(i \int d^{4} x\left[D_{\mu} \phi D^{\mu} \phi^{*}-m^{2} \phi \phi^{*}\right]\right)
$$

onde $D_{\mu}=\partial_{\mu}+i e_{b} A_{\mu}$.

Fazendo a integral por partes do lado direito de (A.4), ou seja, a ação, obtemos:

$$
\exp \left(i \int d^{4} x \mathcal{L}_{\text {corr }}\left(A_{\mu}\right)\right)=\int \mathcal{D} \phi \mathcal{D} \phi^{*} \exp \left(-i \int d^{4} x \phi^{*} \hat{D} \phi\right)=\operatorname{det}(\hat{D})^{-1}
$$

abandonando os termos de superfície. O operador $\hat{D}$ é dado por $\hat{D}=D_{\mu} D^{\mu}+m^{2}$.

Sabendo que o determinante em (A.5), pode ser escrito como:

$$
\operatorname{det}(\hat{D})^{-1}=\exp [-\operatorname{Tr} \ln \hat{D}]=\exp [-\langle\mathbf{x}, t|\ln \hat{D}| \mathbf{x}, t\rangle],
$$

em que o traço é calculado na base $|\mathbf{x}, t\rangle$, mesma utilizada por Schwinger em seu trabalho original. Inserindo a forma desse determinante em (A.5), temos que $\mathcal{L}_{\text {eff }}=$ $i\langle\mathbf{x}, t|\ln \hat{D}| \mathbf{x}, t\rangle$. Podemos reescrever $\mathcal{L}_{\text {eff }}$ na representação integral, uma vez que:

$$
\ln \hat{D}=-\int_{0}^{\infty} \frac{d s}{s} \exp [-i(\hat{D}-i \varepsilon) s]
$$

em que tiramos infinitesimalmente a integração do eixo real com $\varepsilon \rightarrow 0^{+}$e $s$ é conhecido como o parâmetro de tempo próprio. Logo, 


$$
\mathcal{L}_{e f f}=-i \int_{0}^{\infty} \frac{d s}{s} \mathrm{e}^{-i\left(m^{2}-i \varepsilon\right) s} K(x, x, s)
$$

em que $K(x, x, s)$ é o kernel dado por $K(x, x, s)=\left\langle\mathbf{x}, t\left|\mathrm{e}^{-i \hat{H} s}\right| \mathbf{x}, t\right\rangle$.

Convém salientar que as condições de contorno estão implícitas em (A.8), uma vez que a integração do tempo próprio é feita no intervalo $[0, \infty]$ ao invés de $[-\infty, \infty]$.

Podemos nesse ponto utilizar o kernel para calcular a função de Green, que também é de muito interesse para o problema. A função de Green é dada por:

$$
G\left(x, x^{\prime}\right)=i \int_{0}^{\infty} d s\left\langle\mathbf{x}, t\left|\mathrm{e}^{-i \hat{H} s}\right| \mathbf{x}, t\right\rangle,
$$

Essa função de Green também tem as mesmas condições de contorno da integração no intervalo $[0, \infty]$, o que garante que $G$ é a função de Feynman em quatro dimensões.

Determinado o formalismo, temos que calcular o kernel para $\hat{H}$ dado para podermos calcular a Lagrangeana efetiva. O calibre que vamos utilizar é $A^{\mu}=(0,0,0, A(t))=$ $(0,0,0,-E t)$, para podermos calcular o efeito Schwinger.

Podemos escrever $\hat{H}$, utilizando (5.67), que inserindo a expressão acima na expressão do kernel, temos que:

$$
K(x, x, s)=\left\langle x\left|e^{\left(-i \frac{P_{0} P_{3}}{e E}\right)} e^{-i s\left[P_{0}^{2}-P_{\perp}^{2}-e^{2} E^{2} t^{2}\right]} e^{\left(i \frac{P_{0} P_{3}}{e E}\right)}\right| x\right\rangle,
$$

e introduzindo conjuntos completos de estados do momento e resolvendo as integrais, nos momentos $\mathbf{P}$ e $\mathbf{P}^{\prime}$, calculamos o traço para bósons sem considerarmos o termo proporcional ao campo eletromagnético em (5.66). Para resolver a integral que surge no cálculo do traço, utilizamos o mesmo método utilizado na Seção 5.2.1.

Assim, o kernel é dado de forma simples por:

$$
\begin{gathered}
K(x, x, s)=\frac{e E}{2(2 \pi)^{2}} \int d \omega\left\langle\omega\left|e^{i s\left(P_{0}^{2}-e^{2} E^{2} X_{0}^{2}\right)}\right| \omega\right\rangle \\
=\frac{1}{2} \frac{1}{\operatorname{senh}(e E s)} .
\end{gathered}
$$

Logo, de (A.8), a Lagrangeana efetiva para bósons em um campo elétrico constante é:

$$
\mathcal{L}_{\text {eff }}^{b}=\frac{1}{16 \pi} \int_{0}^{\infty} \frac{d s}{s^{2}} \mathrm{e}^{-i m^{2} s} \frac{e_{b} E}{\operatorname{senh}\left(e_{b} E s\right)} .
$$

Fazendo o mesmo procedimento para férmions, em que $\hat{H}_{f}=\left(i \gamma_{\mu} \partial^{\mu}-e \gamma_{\mu} A^{\mu}\right)^{2}$ e calculando o traço por (5.66), temos que o kernel pode ser obtido:

$$
\begin{aligned}
K_{f}(x, x, s) & =\left\langle x\left|e^{\left(-i \frac{P_{0} P_{3}}{e E}\right)} e^{-i s\left[P_{0}^{2}-P_{\perp}^{2}-e^{2} E^{2} t^{2}+\frac{e}{2} \sigma_{\mu \nu} F^{\mu \nu}\right]} e^{\left(i \frac{P_{0} P_{3}}{e E}\right)}\right| x\right\rangle \\
& =4 \cosh (s e E)\left\langle x\left|e^{\left(-i \frac{P_{0} P_{3}}{e E}\right)} e^{-i s\left[P_{0}^{2}-P_{\perp}^{2}-e^{2} E^{2} t^{2}\right]} e^{\left(i \frac{P_{0} P_{3}}{e E}\right)}\right| x\right\rangle
\end{aligned}
$$


uma vez que $\operatorname{tr} \exp \left(\frac{i s e}{2} \sigma_{\mu \nu} F^{\mu \nu}\right)=4 \cosh (s e E)$. Assim, fazendo o mesmo procedimento feito para bósons e fazendo a analogia com o oscilador harmônico, temos que o kernel para o campo fermiônico é dado por:

$$
K_{f}(x, x, s)=2 \operatorname{coth}(s e E)
$$

A Lagrangeana efetiva para férmions em um campo elétrico constante é dado por:

$$
\mathcal{L}_{\text {eff }}^{f}=-\frac{e E}{8 \pi} \int_{0}^{\infty} \frac{d s}{s^{2}} \mathrm{e}^{-i m^{2} s} \operatorname{coth}(s e E) .
$$

Essas Lagrangeanas efetivas representam a resposta do vácuo quântico a presença do campo externo. Com isso, podemos calcular a probabilidade do sistema não emitir pares. Em função da ação de um problema, essa probabilidade é dada por $P_{0}=\left|e^{i S_{e f f}}\right|^{2}$, e como $S_{\text {eff }}$ contém partes reais e imaginárias, seu módulo ao quadrado corresponde a:

$$
P_{0}=e^{-2 I m S_{e f f}}
$$

Como a probabilidade acima pode ser escrita em termos de uma outra probabilidade $W, P_{0}=e^{W}$, de acordo com (A.3) podemos escrever $\omega=2 \operatorname{Im} \mathcal{L}_{\text {eff }}$, em que $\omega$ é a probabilidade $W$ por unidade de volume e de tempo. Logo, provamos aqui, como já havíamos afirmado anteriormente, que a parte imaginária da Lagrangeana efetiva que contabiliza a criação de partículas.

Para calcularmos a parte imaginária das Lagrangeanas efetivas acima, temos primeiramente que fazer a integral no tempo próprio em (A.12) e (A.15). Seus integrandos têm singularidades em $s_{n}=n \pi i / e E$, com $n=\ldots-1,0,1, \ldots$ Escolhendo o contorno de integração no semiplano inferior complexo de $s$, então temos que:

$$
2 \operatorname{Im}_{\text {eff }}^{(b, f)}=-\frac{1}{8 \pi} \sum_{n=1}^{\infty} \frac{(\mp 1)^{n+1}}{s_{n}^{2}} \exp \left(-\frac{n \pi m^{2}(b, f)}{e_{(b, f)^{E}}^{E}}\right)
$$

em que o termo em $n=0$ foi removido para que $\mathcal{L}_{\text {eff }}^{(b, f)} \rightarrow 0$ quando $E \rightarrow 0$. Com isso, retomamos o resultado calculado via teoria de espalhamento para bóson:

$$
\omega_{b}=\frac{\alpha E^{2}}{2 \pi^{2}} \sum_{n=1}^{\infty} \frac{(-1)^{n+1}}{n^{2}} \exp \left(-\frac{n \pi m^{2}}{|e E|}\right)
$$

e, para férmions,

$$
\omega_{F}=\frac{\alpha E^{2}}{\pi^{2}} \sum_{n=1}^{\infty} \frac{1}{n^{2}} \exp \left(-\frac{n \pi m^{2}}{|e E|}\right) .
$$

Utilizamos aqui a técnica da Lagrangena efetiva para o cálculo da probabilidade de 
não emitir pares. Contudo, essa técnica é muito poderosa e permite fazer quaisquer possíveis correções de divergências e consequentes renormalizações, presentes no sistema. Não faremos essa análise aqui, mas pode ser vista em [167]. Contudo, essa técnica limita a forma do campo externo aplicado, uma vez que o cálculo da Lagrangeana efetiva pode ser muito difícil dependendo da forma do potencial eletromagnético. Logo, a técnica baseada na Lagrangeana efetiva não pode ser usada para quaisquer interação com campos externos, uma vez que para potenciais ligeiramente diferentes do usado aqui sua determinação é desconhecida. 


\section{Apêndice B}

\section{Integração complexa}

Para fazermos essa integral é necessário fazer uma análise de suas características. A expressão em $c$ contém um fator oscilatório muito rápido com freqüências da ordem de $m / \omega_{0}$, o que requer a aplicação do método steepest-descent ou método do gradiente descendente no eixo complexo $x$. Esse método é utilizado para localizar o mínimo local mais próximo e será utilizado aqui, uma vez que na vizinhança do ponto de interesse temos pólos e branch points. O intervalo $-\pi \leq \operatorname{Re} x \leq \pi$, tem quatro branch points originados dos zeros de $\left\{m^{2}+p_{\perp}^{2}+\left[p_{3}-\left(e E_{0} / \omega_{0}\right) \cos \omega_{0} t\right]^{2}\right\}^{1 / 2}$, denotados por $\pm x_{0}$ e $\pm \bar{x}_{0}$, de forma que as partes real e imaginária de $x_{0}$ são positivas:

$$
\left(p_{3}-\frac{e E_{0}}{\omega_{0}} \cos \omega_{0} t\right)=i\left(m^{2}+p_{\perp}^{2}\right)^{1 / 2}, 0 \leq \operatorname{Re} x_{0} \leq \pi / 2, \operatorname{Im} x_{0}>0
$$

Assumimos que $p_{3}>0$, uma vez que $|c|^{2}$ é uma função par de $p_{3}$.
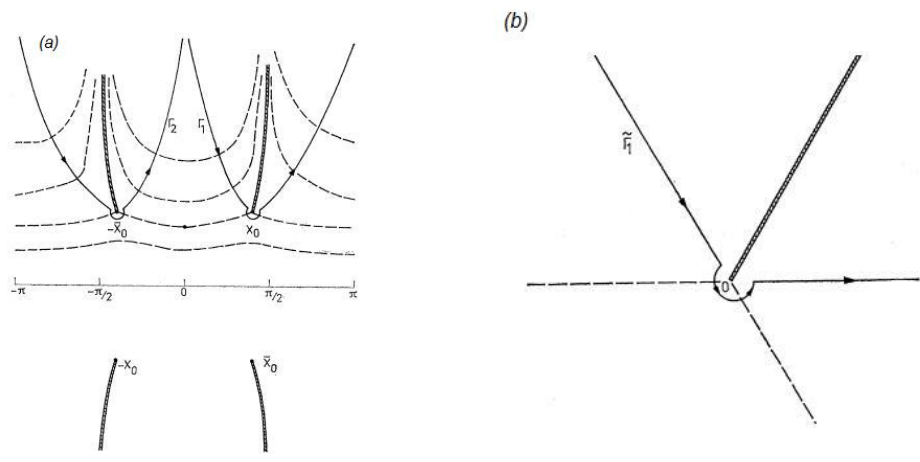

Figura B.1: (a)Esquema da linhas da função representada no primeiro termo da multiplicação em $c_{1}$, necessárias para o cálculo da probabilidade. Os contornos de integração são $\Gamma_{1}$ e $\Gamma_{2}$. (b) Contorno de integração $\widetilde{\Gamma}_{1}$. Figuras retiradas de [74].

Da periodicidade da função, vamos obter $c$, permitindo que os pontos finais de inte- 
gração sejam $-\pi+i \lambda$ e $\pi+i \lambda$, com $\lambda$ real e arbitrário.

Para obter o melhor contorno de integração vemos na Figura B.1 que o caminho deve evitar $x_{0}$ e terminar nos vales seguindo as duas linhas descendentes. O contorno é feito, então, em duas partes, uma ao redor de $x_{0}$ e outra ao redor de $-\bar{x}_{0}$, identificados na Figura B.1 por $\Gamma_{1}$ e $\Gamma_{2}$. Sendo $c_{1}$ e $c_{2}$ as contribuições de cada um desses contornos, com $c_{2}=-\bar{c}_{1}$, então $c=2 i \operatorname{Im} c_{1}$, em que:

$$
\begin{aligned}
c_{1} & \simeq \exp \left\{\frac{-2 i}{\omega_{0}} \int_{0}^{x_{0}} d x^{\prime}\left[m^{2}+p_{\perp}^{2}+\left(p_{3}-\frac{e E_{0}}{\omega_{0}} \cos \omega_{0} t\right)^{2}\right]^{1 / 2}\right\} \\
& \times \frac{\omega_{0}}{2 e E} \int_{\Gamma_{1}} \frac{d x}{2 \pi x} \exp \{\frac{2}{3} \underbrace{\frac{2 i}{\omega_{0}}\left[2 i\left(m^{2}+p_{\perp}^{2}\right)^{1 / 2} \frac{e E}{\omega_{0}} \operatorname{sen} x_{0}\right]^{1 / 2}}_{\nu} x^{3 / 2}\} .
\end{aligned}
$$

Fazendo uma mudança de variáveis na integral no contorno, podemos simplificá-la :

$$
\int_{\widetilde{\Gamma}_{1}} \frac{d y}{2 \pi y} \exp \left(y^{3 / 2}\right),
$$

que pelo mapeamento $u=y^{3 / 2}$, o contorno é transformado em um circuito positivo em torno da origem terminando em $-\infty$, na Figura B.1b. Assim, $\int_{\widetilde{\Gamma}_{1}} \frac{d y}{2 \pi y} \exp \left(y^{3 / 2}\right)=$ $\frac{2}{3} \oint \frac{d u}{2 \pi u} e^{u}=\frac{2}{3} i$. 


\section{Apêndice C}

\section{Funções de Hankel}

Apresentamos aqui as expansões assintóticas das funções de Hankel de primeira e segunda espécies, sendo $x \equiv-k \eta$, com $k>0$ e $\eta \in(-\infty, 0)$.

Para $x \rightarrow \infty$ :

$$
\begin{aligned}
& H_{\nu}^{(1)}(x) \approx \sqrt{\frac{2}{\pi x}} \mathrm{e}^{i\left(x-\nu \frac{\pi}{2}-\frac{\pi}{4}\right)}, \\
& H_{\nu}^{(2)}(x) \approx \sqrt{\frac{2}{\pi x}} \mathrm{e}^{-i\left(x-\nu \frac{\pi}{2}-\frac{\pi}{4}\right)} .
\end{aligned}
$$

Para $x \rightarrow 0$ :

$$
\begin{aligned}
& H_{\nu}^{(1)}(x) \approx-i \frac{2^{\nu-1}}{\pi} \frac{\Gamma(\nu)}{\Gamma(3 / 2)} x^{-\nu} \\
& H_{\nu}^{(2)}(x) \approx i \frac{2^{\nu-1}}{\pi} \frac{\Gamma(\nu)}{\Gamma(3 / 2)} x^{-\nu}
\end{aligned}
$$

